\title{
Engineering of a NIR fluorescent protein for live-cell nanoscopy
}

\author{
Dissertation \\ for the award of the degree \\ "Doctor rerum naturalium" \\ within the doctoral program \\ Biomolecules: Structure - Function - Dynamics \\ of the Georg-August University School of Science (GAUSS)
}

submitted by

\section{Florian Habenstein}

Göttingen, 2020 



\section{Thesis Committee:}

Prof. Dr. Stefan Jakobs (Referee)

Clinic of Neurology, University Medical Center, Göttingen

Mitochondrial Structure and Dynamics Group

Department of NanoBiophotonics

Max Planck Institute for Biophysical Chemistry, Göttingen

Prof. Dr. Dirk Schwarzer (2 ${ }^{\text {nd }}$ Referee)

Department of Dynamics at Surfaces

Max Planck Institute for Biophysical Chemistry, Göttingen

Prof. Dr. Dr. h.c. mult. Stefan W. Hell

Department of Nanobiophotonics

Max Planck Institute for Biophysical Chemistry, Göttingen

Further members of the Examination Board:

Prof. Dr. Tiago Outeiro

Department of Experimental Neurodegeneration

University Medical Center, Göttingen

Prof. Dr. Michael Müller

Institute of Neuro- and Sensory Physiology

University Medical Center, Göttingen

Prof. Dr. Alexander Egner

Optical Nanoscopy

Laser Laboratory, Göttingen

\section{Date of oral examination:}

$3^{\text {rd }}$ September 2020 



\section{Abstract}

Since its first use as a genetically encoded fluorescent marker in 1994, GFP and its homologues have fundamentally revolutionized live-cell fluorescence imaging and became an essential tool for biomedical research. Many fluorescent proteins have been engineered from GFP-like proteins with excitation and emission covering the entirety of the visible spectrum. However, despite substantial efforts it was not possible to reach the near infrared (NIR) spectral region beyond $650 \mathrm{~nm}$ with a GFP derived fluorescent protein.

The NIR spectral region between 650 to $900 \mathrm{~nm}$ is especially suited for live-cell deep-tissue fluorescence imaging due to reduced autofluorescence, light scattering and absorption of tissue in this spectral region. Additionally, photo toxicity of NIR light is reduced compared to UV/vis light.

Recently, utilization of engineered bacterial phytochrome variants opened the NIR spectral region for fluorescence microscopy with genetically encoded fluorescent markers. Bacterial phytochromes gain their unique optical properties by incorporating the external chromophore and heme degradation product biliverdin which is ubiquitous in mammalian cells. A variety of bacterial phytochrome based fluorescent proteins has been successfully applied in various fluorescence microscopy techniques. However, to date all engineered bacterial phytochrome variants absorbing and emitting beyond $650 \mathrm{~nm}$ suffer from a short fluorescence lifetime and a low fluorescence quantum yield, limiting their potential for fluorescence microscopy.

In this work, an automated fluorescence lifetime screening microscope was built and applied to increase the fluorescence lifetime and quantum yield of the engineered fluorescent protein miRFP703 via directed evolution. The final protein variant (V410) had a fluorescence lifetime of $1.1 \mathrm{~ns}$ and a fluorescence quantum yield of $21 \%$. With this it is the brightest NIR fluorescent protein described to date. V410 exhibited a good $\mathrm{pH}$ stability and a high extinction coefficient. In live-cell fluorescence microscopy, V410 performed well as fusion tag for various cellular structures. With STED microscopy, resolutions well beyond $80 \mathrm{~nm}$ down to $40 \mathrm{~nm}$ were measured on endogenously tagged vimentin filaments. In consecutive confocal and STED recordings, with 1000 and 100 consecutive frames, respectively, V410 demonstrated superior photo stability. Utilizing the fluorescence lifetime difference between V410 and the template miRFP703 of approximately 400 ps, two-color fluorescence lifetime confocal and STED imaging was performed entirely in the NIR spectral region with a single excitation beam at $660 \mathrm{~nm}$ and a STED beam at $820 \mathrm{~nm}$. 



\section{Acknowledgements}

I would like to thank all people who made this work possible with their help and support. First, I want to thank Prof. Dr. Stefan Jakobs and Prof. Dr. Stefan Hell for the opportunity to work on my doctoral thesis in this fantastic environment of the department of NanoBiophotonics. I would like to thank Prof. Dr. Stefan Jakobs for his supervision, guidance and support during the course of this work. I am grateful to Prof. Dr. Dirk Schwarzer and Prof. Dr. Stefan Hell for their advice and support in my thesis advisory committee. Furthermore, I am grateful to the members of my extended examination board Prof. Dr. Tiago Outeiro, Prof. Dr. Michael Müller and Prof. Dr. Alexander Egner.

I am especially grateful to Dr. Nickels Jensen for countless discussions, practical help, mental support and essential guidance.

I want to thank Dr. Alexey Chizhik for carrying out the fluorescence quantum yield measurements and Dr. Marcel Leutenegger for his essential theoretical and practical support in the FLIM project. I am grateful to Dr. Jessie Qin-Dregely and Dr. Andreas Fognini, Single Quantum B.V., Delft, The Netherlands, for the equipment demonstration of superconducting nanowire detectors in the visible range and Dr. Iman Esmaeil Zadeh, Delft University of Technology, Delft, The Netherlands, for support with customized superconducting nanowire detectors for fluorescence lifetime imaging. For his practical help I want to thank Michael Weber. I am also thankful to Dr. Jan Keller for always being happy to share his knowledge and insights about microscopy and image analysis.

For their excellent support in the lab, I want to thank Sylvia Löbermann, Tanja Gilat, Rita Schmitz-Salue and Ellen Rothermel. Furthermore, I want to thank Cornelia Heistermann for keeping the institute in good condition. I would like to thank Jaydev Jethwa, Simon Piechota and Marco Roose for their technical support and Stefanie Kruse, Sarah Leondarakis and Janine Ehlert for helping with organizational problems.

I am very grateful for all the great colleagues from the department of NanoBiophotonics with whom I had the pleasure to work with during my doctoral thesis. Many of you became good friends to me. I want to thank my current and former office mates Axel Rösch, Sarah Vanessa Schweighofer, Sebastian Schnorrenberg and Grazvydas Lukinavicius for a fantastic time in and outside the office. I would like to thank Christian Brüser for keeping me fit by forcing me to the Gym. Thanks to the people with whom I shared the good and bad times of my $\mathrm{PhD}$ experience: Nickels Jensen, Daniel Stumpf, Timo Konen, Felix Kleißler, Isabelle Jansen, Jasmin Hubrich, Phillip Alt, Felix Lange, Till Stephan, Christian Stuut, Jasmin Pape and many others.

For proofreading this thesis I would like to thank Nickels Jensen, Felix Kleißler, Christian Brüser and Jan Keller.

I want to thank my family and friends for their support. Most of all, I want to thank you, Clara, for always being loving, supportive and understanding during the time of my doctoral thesis and beyond. Thank you for sticking with me despite hard times and physical distance. 



\section{Contents}

$\begin{array}{lll}\text { Abstract } & \text { v }\end{array}$

Acknowledgements vii

1 Introduction $\quad \mathbf{1}$

1.1 Fluorescence Microscopy . . . . . . . . . . . . . . . . . 1

1.1.1 Physical Characteristics of Fluorescence . . . . . . . . . 2

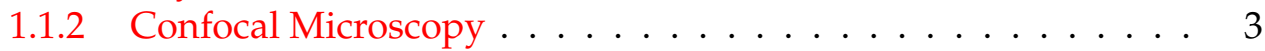

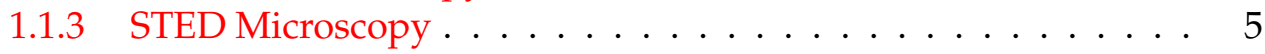

1.2 Fluorescent Labels . . . . . . . . . . . . . . . . . 6

1.2.1 GFP-like fluorescent Proteins ............. 7

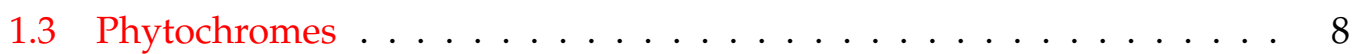

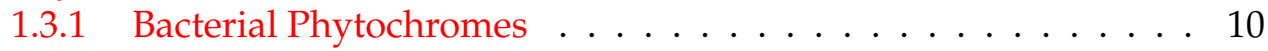

1.3.2 Phytochrome based Fluorescent Proteins . . . . . . . . . 15

1.4 Aim of the Study . . . . . . . . . . . . . . . 17

2 Material and Methods $\quad 19$

2.1 Molecular Biology ...................... 19

2.1.1 Standard Cloning . . . . . . . . . . . . . . . 19

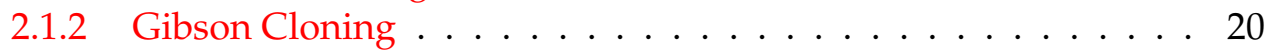

2.1 .3 Site Directed Mutagenesis . . . . . . . . . . . . . . 20

2.1.4 Multiple Site Mutagenesis ............... . 20

2.1.5 Error Prone Mutagenesis .............. 21

2.2 Expression Systems . . . . . . . . . . . . . . . . . 21

2.2.1 Bacterial Strains . . . . . . . . . . . . . . . 22

2.2.2 Cloning of Plasmids for Bacterial Expression . . . . . . . . . 22

2.2.3 Mammalian Cell Lines . . . . . . . . . . . . . . . . . 22

2.2.4 Cloning of Plasmids for Transient Mammalian Expression . . . 22

2.2.5 Cloning of Plasmids for Stable Mammalian Cell Lines . . . . . . 24

2.3 Cell Culture . . . . . . . . . . . . . . . . . . . 24

2.3.1 Creation of Stable Mammalian Cell Lines . . . . . . . . . . . . 25

2.4 Protein Expression and Purification . . . . . . . . . . . . . . . 25

2.4.1 Transformation of Bacterial Cells . . . . . . . . . . . . 25

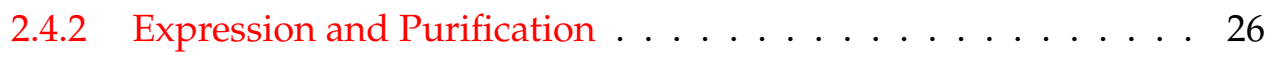

2.5 Protein Characterization . . . . . . . . . . . . . 27

2.5.1 Measurement of Absorption Spectra . . . . . . . . . . . . . 27

2.5.2 Measurement of Excitation and Emission Spectra . . . . . . . . 27

2.5.3 Measurement of Fluorescence Lifetime . . . . . . . . . . . . 27

2.5.4 Measurement of Fluorescence Quantum Yield . . . . . . . . . . 27

2.5.5 Measurement of pH Stability .............. . . 28

2.5.6 Semi-native Gel Electrophoresis . . . . . . . . . . . 28

2.5.7 Size Exclusion Chromatography ............. 28

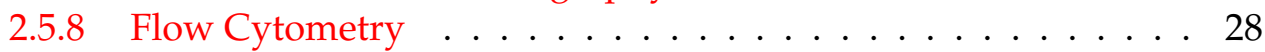


2.6 Fluorescence Lifetime Screening . . . . . . . . . . . . . . . . . . 29

2.6.1 Automated Fluorescence Lifetime Screening Microscope . . . . 29

2.6 .2 Directed Evolution . . . . . . . . . . . . . . . . . . . 29

2.7 Microscopy. . . . . . . . . . . . . . . . . . . . . . . . 31

2.7.1 Image Processing and Analysis . . . . . . . . . . . . . 32

3 Results 35

3.1 Implementation of Fluorescence Lifetime Screening . . . . . . . . . . 35

3.2 Directed Evolution of miRFP703 to Increase Lifetime and Quantum

Yield . . . . . . . . . . . . . . . . 37

3.3 Biophysical Characterization of V410 . . . . . . . . . . . . . . 42

Optical Characterization of V410 . . . . . . . . . . 42

pH Stability of V410 . . . . . . . . . . . . . . . 43

Oligomeric State of V410 . . . . . . . . . . . . . . . . 44

Characterization of Brightness of V410 in Mammalian Cells . . 44

3.4 Application of V410 in Live-Cell Imaging . . . . . . . . . . . . . . . . . . 48

3.4 .1 Confocal Imaging . . . . . . . . . . . . . . . . . . . 48

Confocal Movies . . . . . . . . . . . . . . . . . 50

3.4.2 Implementation of STED Imaging . . . . . . . . . . . . . . 50

Correlation between Photo Bleaching and STED Wavelength . . 51

Correlation between Reexcitation and STED Wavelength . . . . 51

Correlation between STED Efficiency and STED Wavelength . . 53

3.4 .3 STED Imaging . . . . . . . . . . . . . . . . . . . . 55

STED Movies . . . . . . . . . . . . . . . . . . 59

3.4.4 Fluorescence Lifetime Imaging . . . . . . . . . . . . . . . . . 60

Decomposition Methods . . . . . . . . . . . . . . . . 6 60

Evaluation of Decomposition Methods . . . . . . . . . . . 63

Two-color FLIM imaging . . . . . . . . . . . . . 64

$\begin{array}{lll}4 & \text { Discussion } & \mathbf{6 7}\end{array}$

4.1 Directed Evolution . . . . . . . . . . . . . . . . . . 67

4.1.1 Screening and Selection . . . . . . . . . . . . . 67

4.1 .2 Positions . . . . . . . . . . . . . . . . . . . 69

Position $193 \ldots \ldots \ldots$. . . . . . . . . . . . . . 69

Position $202 \ldots \ldots \ldots$

Position $210 \ldots \ldots \ldots \ldots$

Position $253 \ldots \ldots \ldots \ldots \ldots$

4.2 Characterization . . . . . . . . . . . . . . . 71

Fluorescence Quantum Yield . . . . . . . . . . . . . . . 71

4.2.1 Spectral Properties . . . . . . . . . . . . . . . 72

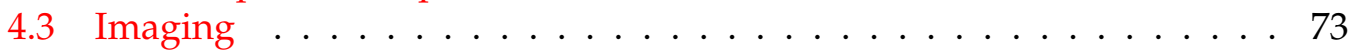

4.3 .1 Confocal Imaging . . . . . . . . . . . . . . . . . 74

Confocal Movies . . . . . . . . . . . . . . . . . 74

4.3 .2 STED Imaging . . . . . . . . . . . . . . . . . . . . . . . . . . . . . . . . . . . .

Implementation . . . . . . . . . . . . . . . . . 75

STED Imaging . . . . . . . . . . . . . . . . . . . . . . . . . . . . . . . . . .

STED Movies . . . . . . . . . . . . . . . . . 76

4.3 .3 FLIM Imaging . . . . . . . . . . . . . . . . . . . . . 76

Decomposition Algorithms . . . . . . . . . . . . . . 77

4.3 .4 Outlook . . . . . . . . . . . . . . . . . 77

Directed Evolution and Screening $\ldots \ldots$. . . . . . . 77 
Imaging of Living Organisms . . . . . . . . . . . . . 78

$\begin{array}{ll}\text { A Cloning Primer } & 79\end{array}$

$\begin{array}{lll}\text { B Buffers and Media } & 81\end{array}$

$\begin{array}{lr}\text { C Python Code } & 83\end{array}$

C.1 Analysis of $\mathrm{pH}$ spectra . . . . . . . . . . . . . . . . . . . 83

C.2 Bleaching Analysis . . . . . . . . . . . . . . . . 84

C.3 Reexcitation . . . . . . . . . . . . . . . . . 86

C.4 Measuring STED Effect . . . . . . . . . . . . . . . . . . . 87

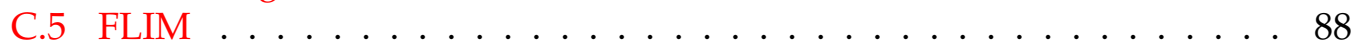

D Microscopy $\mathbf{9 5}$

D.0.1 Microscope Characterization . . . . . . . . . . . . . . 95

D.0.2 Imaging Parameters . . . . . . . . . . . . . . . 96

D.0.3 Line Profiles . . . . . . . . . . . . . . . . . . . . . . . 99

D.0.4 Fluorescence Lifetime Imaging . . . . . . . . . . . . . . 102

$\begin{array}{lr}\text { E Biophysical Characterization } & 105\end{array}$

E.0.1 Flow Cytometry Measurements . . . . . . . . . . . . . . 105

$\begin{array}{lr}\text { Bibliography } & 109\end{array}$

$\begin{array}{ll}\text { Curriculum vitae } & 119\end{array}$ 



\section{List of Figures}

1.1 Correlation between fluorescence brightness and fluorescence quantum yield of different fluorescent proteins . . . . . . . . . . . 3

1.2 Schematic representation of a confocal fluorescence microscope . . . . 4

1.3 Schematic representation of a STED microscope . . . . . . . . . . . 5

1.4 Onedimensional representation of molecules residing in fluorescent state ....................... 6

1.5 Crystal structure of GFP . . . . . . . . . . . . . . . . 7

1.6 Structure of GFP chromophore . . . . . . . . . . . . . . . 8

1.7 Correlation between molecular brightness and emission wavelength of GFP-like fluorescent proteins . . . . . . . . . . . . . . . . 8

1.8 Absorbance in the visible and NIR spectral region of water, haemoglobin and oxyhaemoglobin . . . . . . . . . . . . . . . 10

1.9 Structure of bacterial phytochrome . . . . . . . . . . . . . 11

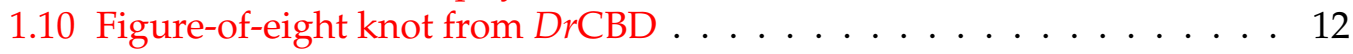

1.11 Interaction between protein scaffold and BV carbonyls . . . . . . . 13

1.12 DIP motif and pyrrole water from DrCBD . . . . . . . . . . . . . 14

1.13 Schematic depiction of phytochrome photo cycle . . . . . . . . . . 14

1.14 Crystal structure of miRFP703 . . . . . . . . . . . . . . . . 16

2.1 Schematic drawing of fluorescence lifetime screening microscope . . . 30

2.2 Workflow of directed evolution approach . . . . . . . . . . . . . 31

2.3 Schematic drawing of NIR STED microscope . . . . . . . . . . . 33

3.1 Fluorescence lifetimes of different NIR FPs measured with the automated screening microscope . . . . . . . . . . . . . . . 36

3.2 Test screen of protein pool consisting of 3 different NIR FPs . . . . . . . 37

3.3 Amino acid site chains within a radius of $5 \AA$ around the BV chromophore in the crystal structure of miRFP703 . . . . . . . . . . . . 38

3.4 Position of mutations indicted on the crystal structure of miRFP703 . . 39

3.5 Comparison between fluorescent lifetimes of protein variants . . . . . 40

3.6 Spectral comparison between protein variants . . . . . . . . . . . . 44 4

3.7 Biophysical characterizations of $\mathrm{V} 410 \ldots \ldots$. . . . . . . . . . . 43

3.8 Characterization of oligomeric state of V410 . . . . . . . . . . . . . 45

3.9 Comparison of flow cytometry data of stable Bxb1 landing pad cell lines 46

3.10 Comparison of flow cytometry data of transient expressions . . . . . 46

3.11 Confocal localisations of cellular structures with V410 . . . . . . . . . 49

3.12 Recording of 1000 consecutive confocal images . . . . . . . . . . . . 50

3.13 Analysis of correlation between photo bleaching and STED wavelength 52

3.14 Analysis of correlation between reexcitation and STED wavelength . . 52

3.15 Analysis of correlation between STED efficiency and STED wavelength 55

3.16 Confocal and STED recordings of genome edited U2OS CRISPR cell line 56

3.17 Confocal and STED recordings of Nup50 . . . . . . . . . . . . . 57

3.18 Confocal and STED recordings of keratin . . . . . . . . . . . 58 
3.19 Confocal and STED recordings of stable Bxb1 landing pad cell line . . 59

3.20 Recording of 100 consecutive STED images . . . . . . . . . . . . . 61

3.21 Reference images and histograms for FLIM . . . . . . . . . . . . 62

3.22 Evaluation of decomposition algorithms with simulated data . . . . . . 64

3.23 Two-color confocal fluorescence lifetime imaging . . . . . . . . . . . . 65

3.24 Two-color STED fluorescence lifetime imaging . . . . . . . . . . . . 65

4.1 Positions 201 and 202 in spectrally distinct miRFP variants . . . . . . 70

4.2 BV binding in miRFP variants . . . . . . . . . . . . . . 71

4.3 Comparison between excitation and emission spectra of V410 and miRFP670. . . . . . . . . . . . . . . . 73

C.1 Schematic representation of matrix equation for least-squares FLIM decomposition method . . . . . . . . . . . . . . . . . 93

D.1 Spectra of excitation and STED beams compared to V410 spectra . . . 95

D.2 Excitation and STED point spread functions . . . . . . . . . . . . 96

D.3 Line profiles of confocal and STED images of genome edited U2OS CRISPR cell line . . . . . . . . . . . . . . . . . . . . . . . . . . 101

D.4 Decomposition of reference FLIM images with maximum likelihood algorithm . . . . . . . . . . . . . . . . . . . 102

D.5 Decomposition of reference FLIM images with least squares algorithm 102

D.6 Count distributions from decomposition of reference FLIM images . . 103

D.7 Accumulated decay histogram FLIM-STED . . . . . . . . . . . . . . 104

E.1 Flow cytometry data of stable Bxb1 landing pad cell lines . . . . . . . 106

E.2 Flow cytometry data of transient expressions . . . . . . . . . . . 107 


\section{List of Tables}

1.1 Engineered NIR fluorescent proteins . . . . . . . . . . . . . . . 15

2.1 PCR protocol . . . . . . . . . . . . . . . . . . . . . . 19

2.2 Multiple site mutagenesis cycler protocol . . . . . . . . . . . . . . 21

2.3 Error prone mutagenesis PCR protocol . . . . . . . . . . . . . . . . . 21

2.4 Summary mammalian constructs for transient expression . . . . . . . . 23

2.5 Summary mammalian constructs for stable integration . . . . . . . . 24

3.1 Comparison of miRFP703 variants . . . . . . . . . . . . . . . 41

3.2 Comparison of flow cytometry data of stable CRISPR cell lines . . . . . 45

3.3 Comparison of flow cytometry data of transient expressions . . . . . 4 47

A.1 Primers . . . . . . . . . . . . . . . . . 79

B.1 Buffers and Media . . . . . . . . . . . . . . . . . . . 81

D.1 Imaging conditions . . . . . . . . . . . . . . . . . . 98

D.2 FWHM of line profiles of confocal and STED images . . . . . . . . . 101 



\title{
List of Abbreviations
}

\author{
A or Ala alanine \\ AU Airy unit \\ a.u. arbitrary units \\ BR bilirubin \\ BV biliverdin \\ C or Cys cysteine \\ CBD chromophore binding domian \\ CenpC1 centromere protein $\mathrm{C} 1$ \\ D or Asp aspartic acid \\ DNA desoxyribonucleic acid \\ DMEM Dulbecco's Modified Eagle Medium \\ E or Glu glutamic acid \\ F or Phe phenylalanine \\ FLIM fluorescence lifetime imaging \\ FP fluorescent protein \\ G or Gly glycine \\ GAF cGMP phosphodiesterase/adenylate cyclase/FhlA \\ GFP green fluorescent protein \\ $\mathrm{H}$ or His histidine \\ HEPES 4-(2-hydroxyethyl)-1-piperazineethanesulfonic acid \\ H2B histone 2 B \\ I or Ile isoleucine \\ K or Lys lysine \\ L or Leu leucine \\ M or Met methionine \\ Map2 microtubule associated protein 2 \\ OD optical density \\ $\mathbf{N}$ or Asn asparagine \\ NA numerical aperture \\ NIR near infrared
}


xviii

\begin{tabular}{|c|c|}
\hline $\begin{array}{l}\text { NTP } \\
\text { Nup50 }\end{array}$ & $\begin{array}{l}\text { nucleoside triphosphate } \\
\text { nuclear pore complex protein } 50\end{array}$ \\
\hline P or Pro & proline \\
\hline PAS & Per ARNT-Sim \\
\hline PBS & phosphate buffered saline \\
\hline РCB & phycocyanobilin \\
\hline PCM & photosensory core module \\
\hline PCR & polymeras cain reaction \\
\hline PHY & phytochrome specific \\
\hline PSF & point spread function \\
\hline РФВ & phytochromobilin \\
\hline Q or Gln & glutamine \\
\hline R or Arg & arginine \\
\hline $\begin{array}{l}\text { S or Ser } \\
\text { STED }\end{array}$ & $\begin{array}{l}\text { serine } \\
\text { stimulated emission depletion }\end{array}$ \\
\hline $\begin{array}{l}\text { T or Thr } \\
\text { TCSPC } \\
\text { tomm } 20\end{array}$ & $\begin{array}{l}\text { threonine } \\
\text { time correlated single photon counting } \\
\text { translocase of outer mitochondrial membrane } 20\end{array}$ \\
\hline UV & ultra violet \\
\hline $\begin{array}{l}\text { V or Val } \\
\text { vis }\end{array}$ & $\begin{array}{l}\text { valine } \\
\text { visible }\end{array}$ \\
\hline W or Trp & tryptophan \\
\hline Y or Tyr & tyrosine \\
\hline
\end{tabular}




\section{Chapter 1}

\section{Introduction}

\subsection{Fluorescence Microscopy}

The earliest example of a light microscope as we define it today dates back to the $17^{\text {th }}$ century. Around 1665 Robert Hooke studied plant cells using an optical system composed of an objective and an eyepiece [59]. From the beginning, the technique of light microscopy revealed biological structures indiscernible to the naked eye. However, it also suffered from two major limitations, namely low contrast and limited resolution. The contrast of classical light microscopy is governed by the optical properties of the sample, mainly absorption and refraction of light. Since absorbance and refractive index differ only slightly within biological samples, the contrast and, therefore, the information content is limited. In the early days, resolution was limited by insufficient instrumentation. Besides that, there is also a theoretical limit of the achievable resolution in a light microscope that was first formulated by Ernst Abbe in 1873 [1]. The theoretical resolution limit is governed by the process of diffraction.

In 1911 Oskar Heimstädt introduced the technique of widefield fluorescence microscopy [51] and used the natural phenomenon of fluorescence to drastically increase the contrast. In fluorescence microscopy, the sample is illuminated with excitation light causing fluorescent molecules in the sample to emit fluorescence light. The fluorescence light can be detected separately from the excitation light. Hence, only light from fluorescent molecules is detected allowing for, in principle, ideal contrast. The first work in which fluorescent dyes were used to specifically enhance the contrast of desired structures was published in 1913 by Stanislaus von Prowazek [98]. Since then, numerous techniques have been developed to specifically label desired cellular structures with fluorescent molecules to enhance the contrast. In practice, however, contrast in widefield fluorescence microscopy of thick biological samples was still not ideal due to out-of-focus fluorescence light. This limitation was tackled with the introduction of confocal fluorescence microscopy by Marvin Minsky in $1955[83,82]$.

The second limitation, the limitation of resolution, remained in place until the $21^{\text {st }}$ century, when two different approaches emerged to overcome the resolution barrier of light microscopy. The approaches are called coordinate-stochastic nanoscopy and coordinate-targeted nanoscopy. The word nanoscopy in this context describes microscopy techniques that are no longer limited in resolution by the diffraction of light. Under coordinate-stochastic nanoscopy techniques like photoactivated localization microscopy (PALM) [9] and stochastic optical reconstruction microscopy (STORM) [112] are subsumed. Techniques like stimulated emission depletion 
(STED) microscopy [53] and reversible saturable optical linear fluorescence transitions (REOLFT) microscopy [57] fall under the label coordinate-targeted nanoscopy. Combining principles of coordinate-stochastic and coordinate-targeted nanoscopy, MINFLUX nanoscopy recently enabled fluorescence imaging and tracking of fluorescent molecules with a precision only limited by the size of the fluorescent molecule itself $[35,5,50]$.

The combination of suitable methods for selectively labeling structures of interest with fluorescent molecules and advanced nanoscopy techniques enable highly sensitive observations of dynamic processes on a molecular level in living biological samples. Such information can only be obtained with fluorescence microscopy, rendering it one of the key techniques in the natural sciences.

\subsubsection{Physical Characteristics of Fluorescence}

The physical basis of fluorescence microscopy is the quantum mechanical phenomenon of fluorescence. An electron in a molecule in the electronic ground state $S_{0}$ can be transferred into the first excited electronic state $S_{1}$ by the absorption of a photon with sufficient energy to bridge the energy gap between the excited and the ground state. The spontaneous emission of light caused by the reverse transition from the excited electronic state $S_{1}$ to the electronic ground state $S_{0}$ is called fluorescence. The energy of the fluorescence photon $E_{P}$ is determined by the energy difference between the excited state $E_{1}$ and the ground state $E_{0}$ as described in equation (1.1). It is always equal or less than the energy of the photon that excited the molecule in the first place.

$$
E_{P}=E_{1}-E_{0}
$$

The mean time a molecule stays in an excited state before the spontaneous emission of a fluorescence photon is called the fluorescence lifetime $\tau$. Spontaneous emission is a probabilistic process and follows an exponential decay as described in equation (1.2).

$$
I(t)=I_{0} e^{-t / \tau}
$$

The quantum yield of fluorescence $\Phi$ is defined as the ratio between the number of absorbed photons and the number of emitted fluorescence photons as described by equation (1.3). The quantum yield of fluorescence is an important parameter to consider when choosing a fluorescent molecule for the application in fluorescence microscopy. Together with the extinction coefficient, it is the most important parameter to determine the contrast achievable with a certain excitation light dose.

$$
\Phi=\frac{\# \text { emitted photons }}{\# \text { absorbed photons }}
$$

The fluorescence quantum yield $\Phi$ and the fluorescence lifetime $\tau$ are connected via equation (1.4) with $k_{\mathrm{r}}$ being the rate constant for radiative decay and $k_{\mathrm{nr}}$ the rate constant for non-radiative decay.

$$
\Phi=\frac{k_{\mathrm{r}}}{k_{\mathrm{r}}+k_{\mathrm{nr}}}=\tau k_{\mathrm{r}}
$$

The fluorescence quantum yield and the fluorescence lifetime of different fluorescent proteins are plotted in figure 1.1. The graph shows an approximately linear 


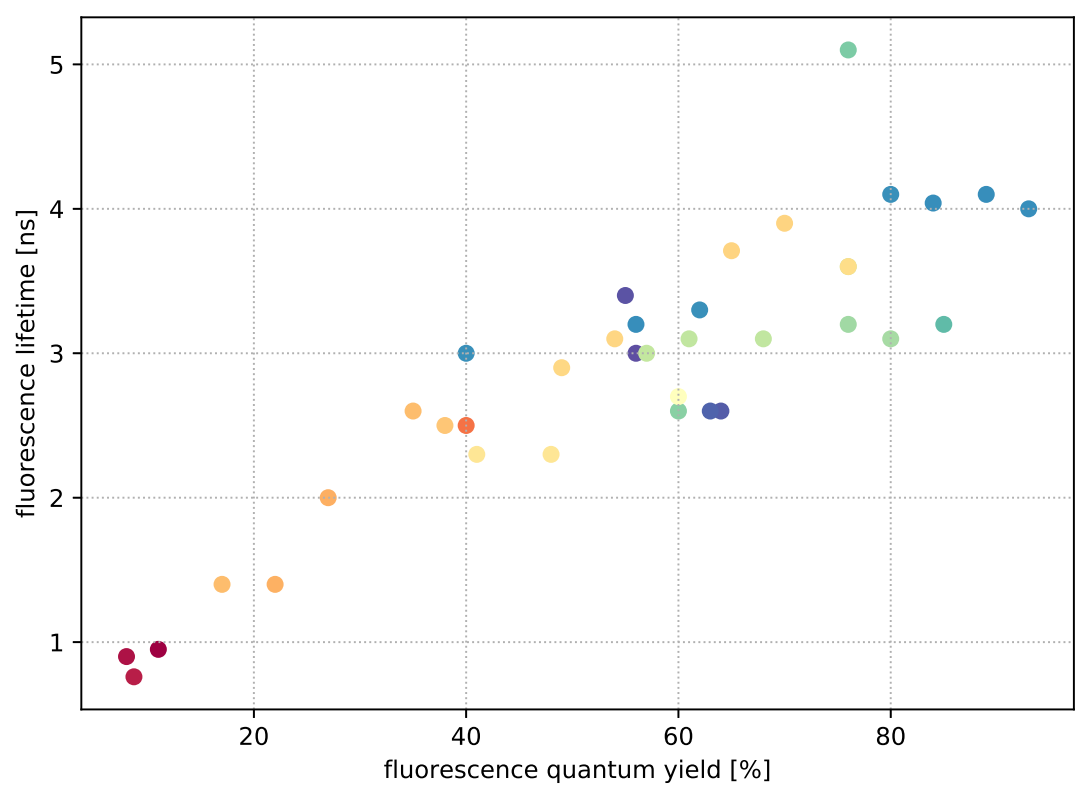

FIGURE 1.1: Correlation between fluorescence lifetime and fluorescence quantum yield of different fluorescent proteins. Data points are colored in correspondence to the emission wavelength of the respective protein. The data were taken from the "Fluorescent Protein Database" [42].

relationship between fluorescence quantum yield and fluorescence lifetime in agreement with equation (1.4). Note that the linear correlation between fluorescence lifetime and fluorescence quantum yield of different fluorescent proteins holds strictly only when the rate constant of radiative decay $k_{\mathrm{r}}$ is constant.

Alternatively to the spontaneous process of fluorescence, the excited state of a molecule can be depopulated by stimulated emission. Stimulated emission can occur when an excited molecule interacts with an incoming photon with a photon energy that is equal to the energy difference between the excited electronic state and the electronic ground state of the molecule. In that case, the excited molecule can be deexcited under the emission of a stimulated photon that is coherent to the first photon. The process of stimulated emission is exploited in STED nanoscopy to overcome Ernst Abbe's resolution limit (see chapter 1.1.3).

\subsubsection{Confocal Microscopy}

The standard optical system for biological imaging today is the confocal fluorescence microscope (see figure 1.2). In confocal fluorescence microscopy the excitation light of a point like emitter is focused by an objective lens into a sample volume. Fluorescence light emitted by the excited sample volume is collected by the objective lens and focused by a tube lens through a pinhole onto a detector. The image of the sample is reconstructed by scanning the beam over the sample and recording the intensity for every sample coordinate.

The pinhole is a core element in a confocal fluorescence microscope. It is positioned in the focal plane of the tube lens and blocks out-of-focus fluorescence light from reaching the detector. Only fluorescence light emitted from the focal spot of 


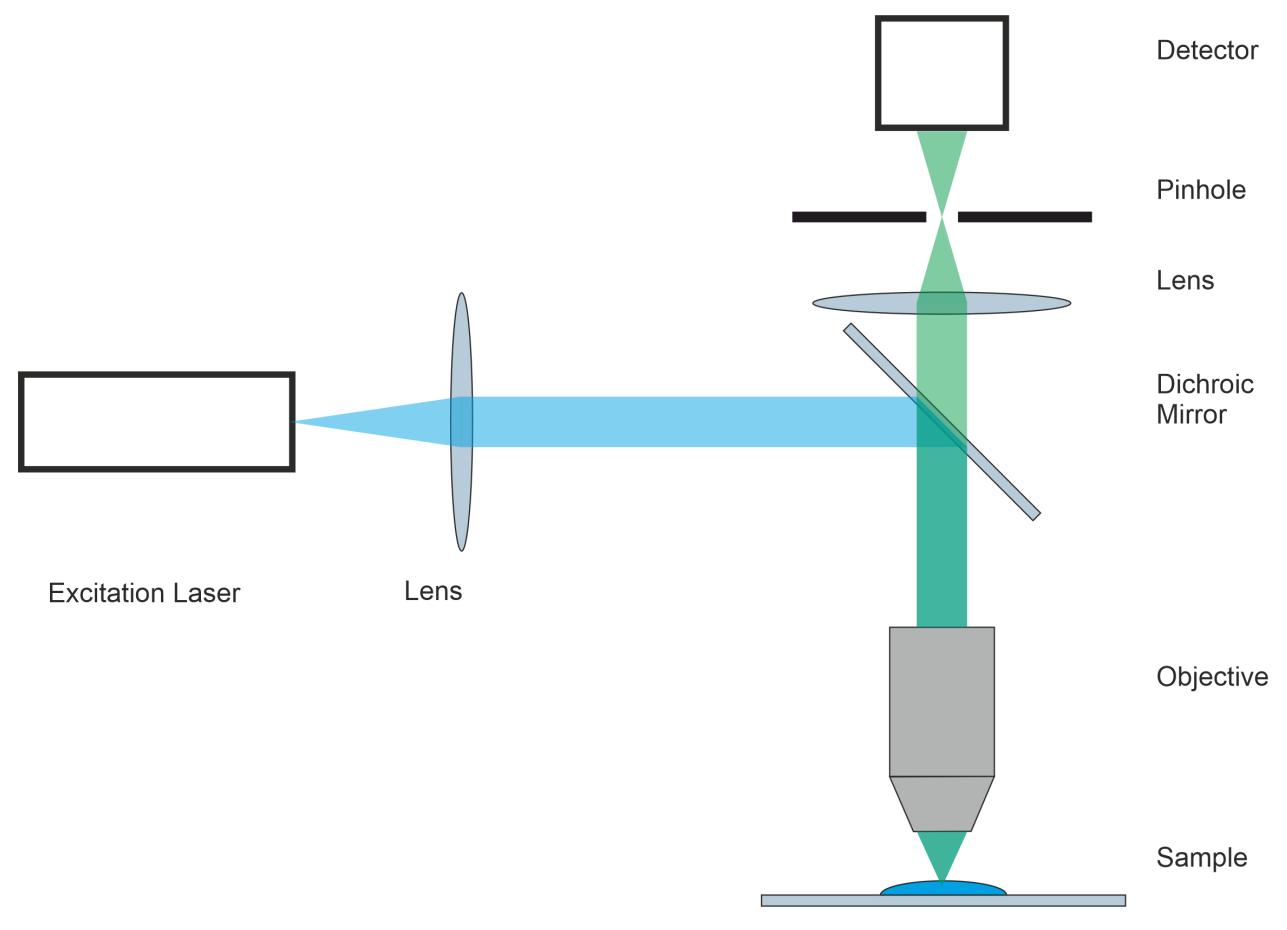

FIGURE 1.2: Schematic representation of a confocal fluorescence microscope.

the objective lens is detected. This drastically increases the contrast in comparison to a widefield fluorescence microscope. In practice, the contrast of a confocal fluorescence microscope is mainly limited by imperfect labeling and autofluorescence from the sample. The term autofluorescence describes a situation where the excitation light does not only excite fluorescence from molecules of interest but also from other molecules present within a biological sample. Molecules that exhibit autofluorescence when imaging with UV/vis light are for example nicotinamid adenine dinucleotide (NADH), flavine adenine dinucleotide (FAD), haemoglobin and oxyhaemoglobin. An approach to reduce the problem of autofluorescence is to avoid imaging in the UV/vis spectral region and use the near-infrared (NIR) spectral region instead (see chapter 1.3.2).

The resolution of a confocal microscope is denoted as the minimal distance between two point like emitters where they can still be recognized as two separate objects. Image reconstruction in confocal microscopy is based on the exact knowledge of the fluorescence signal and the position of the excitation spot. Therefore, the size of the excitation spot determines the resolution of the microscope. Modern fluorescence microscopes have the ability to focus light close to the physical limits. Those limits are goverened by the physical laws of diffraction. The lateral resolution $d_{x y}$ of a perfect widefield fluorescence microscope was described by John William Strutt, 3. Baron Rayleigh in 1896 by equation (1.5) with $\lambda$ being the wavelength of the emitted light and NA the numerical aperture [103].

$$
d_{x y}=\frac{0.61 \lambda}{N A}
$$


Similarly, the axial resolution $d_{z}$ can be described by equation (1.6) with $\mathrm{n}$ being the refractive index of the immersion medium.

$$
d_{z}=\frac{2 \lambda n}{N A^{2}}
$$

For a confocal fluorescence microscope, the lateral and axial resolution is improved by a factor of $\frac{1}{\sqrt{2}} \approx \frac{1}{1.4}$ as compared to equations (1.5) and (1.6) $[25,26]$.

\subsubsection{STED Microscopy}

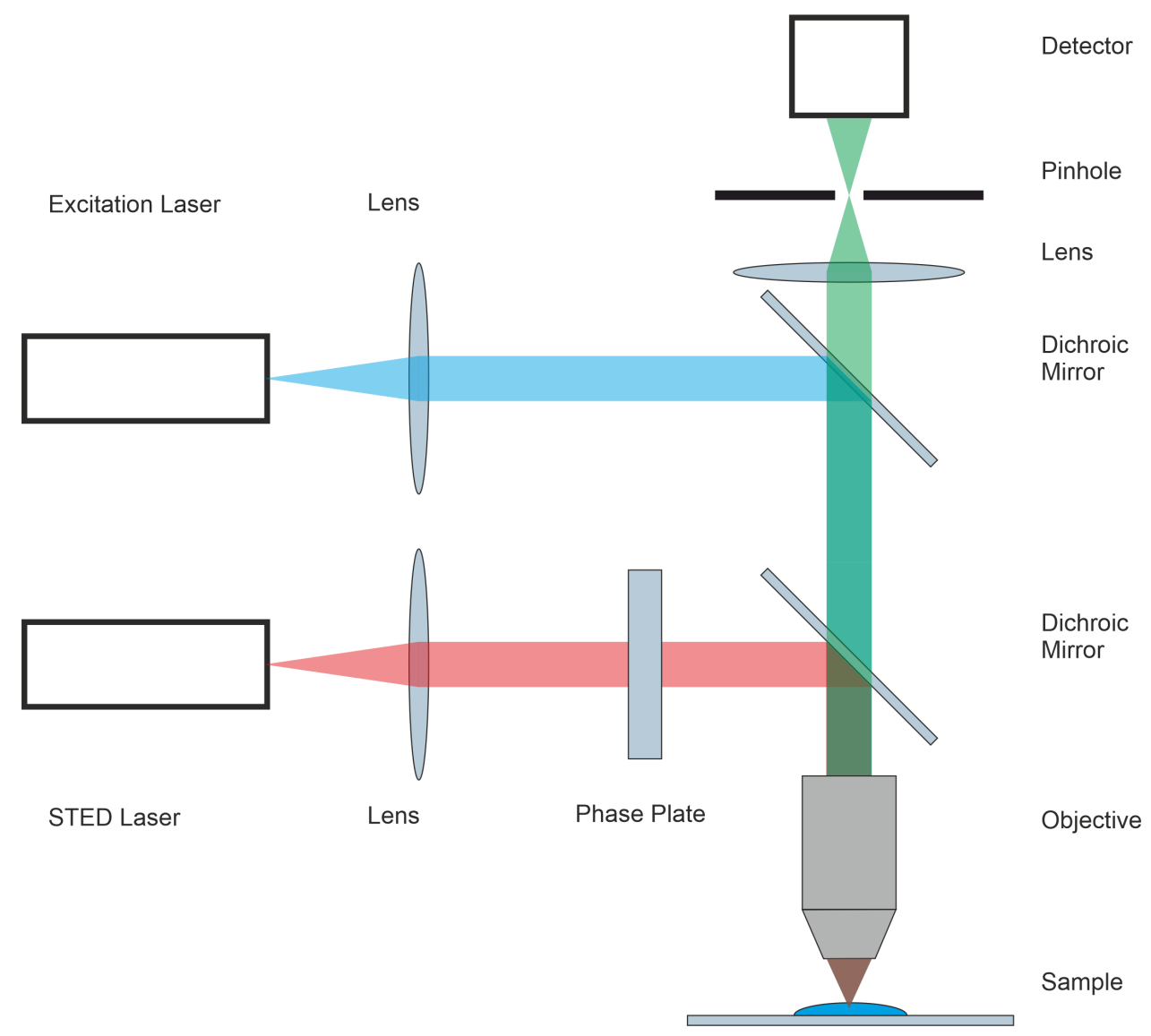

FIGURE 1.3: Schematic representation of a STED microscope.

In STED microscopy the effective size of the excitation volume is drastically reduced as compared to conventional confocal fluorescence microscopy and with this the achievable resolution is increased beyond the limits described by equations 1.5 and 1.6. A schematic drawing of a STED microscope can be seen in figure 1.3.

Reduction of the effective spot size is achieved by superimposing a diffraction limited excitation pattern with a second diffraction limited light pattern that exhibits a minimal intensity region in the center of the excitation pattern. In practice, most of the times this is achieved by superimposing a Gaussian-shaped excitation beam with a donut-shaped STED beam. The wavelength of the donut-shaped STED beam is chosen in a way that molecules in the periphery of the donut are depleted by stimulated emission. Those stimulated photons are separated with a dichroic mirror 


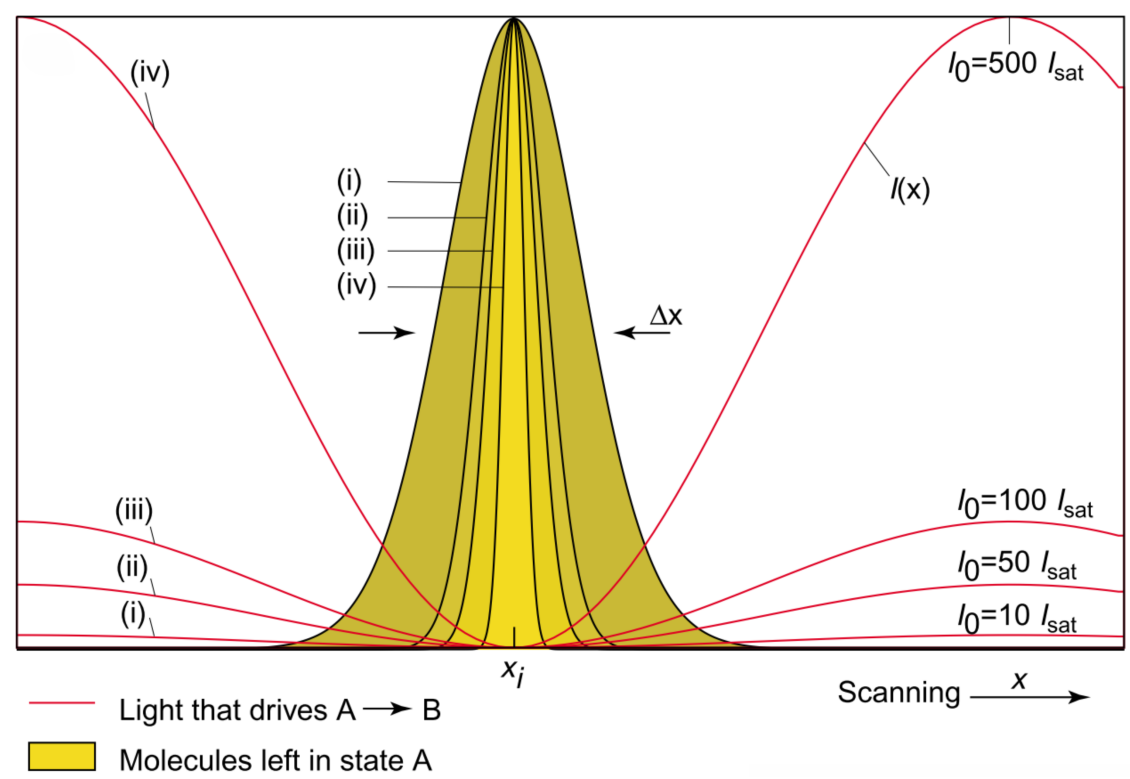

FIGURE 1.4: Onedimensional representation of molecules residing in a fluorescent state confined by different multiples of $I_{\text {sat }}(\mathrm{i}, \mathrm{ii}, \mathrm{iii}, \mathrm{iv})$ at the position $x_{i}$. The figure was adapted from [52].

and do not participate in image formation. Only molecules in the center of the donut beam where the intensity of the depletion beam is below a certain threshold are not depleted by stimulated emission and emit fluorescence photons spontaneously. Only those spontaneously emitted fluorescence photons are detected. The principle is illustrated in figure 1.4. The red curves depict the intensity profiles of the donutshaped depletion beams at different beam intensities. The colored profiles in the center depict the resulting effective excitation spots corresponding to the different STED intensities. To describe the axial resolution $d_{x y}$ of a STED microscope, Abbe's equation can be modified to equation (1.7) with $I_{\text {STED }}$ being the intensity of the STED beam, $I_{\text {sat }}$ being the saturation intensity, which is the intensity where the probability of spontaneous emission is reduced by half, $\sigma$ being the stimulated emission cross section, $\tau$ being the fluorescence lifetime, $h$ being the Planck constant and $v$ being the frequency of the light.

$$
d_{x y}=\frac{\lambda}{2 N A \sqrt{1+\frac{I_{\mathrm{STED}}}{I_{\mathrm{sat}}}}}=\frac{\lambda}{2 N A \sqrt{1+\frac{\sigma \tau I_{\mathrm{STED}}}{h v}}}
$$

In contrast to equation (1.5), the axial resolution $d_{x y}$ in equation (1.7) can in principle be made arbitrarily small by increasing the intensity of the STED beam $I_{S T E D}$. In reality, resolutions down to $30 \mathrm{~nm}$ in biological specimen are possible with STED microscopy.

\subsection{Fluorescent Labels}

In order to make cellular structures visible with fluorescence microscopy, it is necessary to label the structure of interest with fluorescent molecules. This is typically done using either chemically synthesized fluorescent molecules coupled to antibodys [48, 10], nanobodys [86, 95, 81] or ligands [76], or fluorescent proteins. In the 


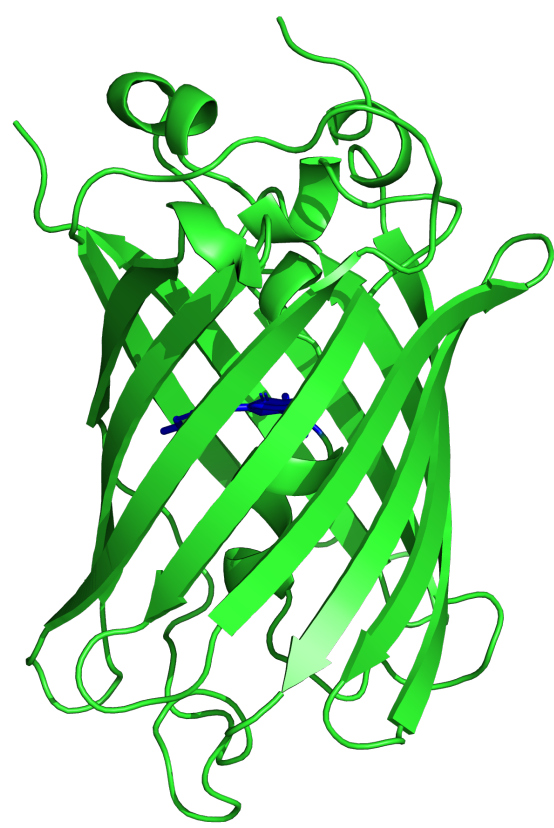

(A) Side view

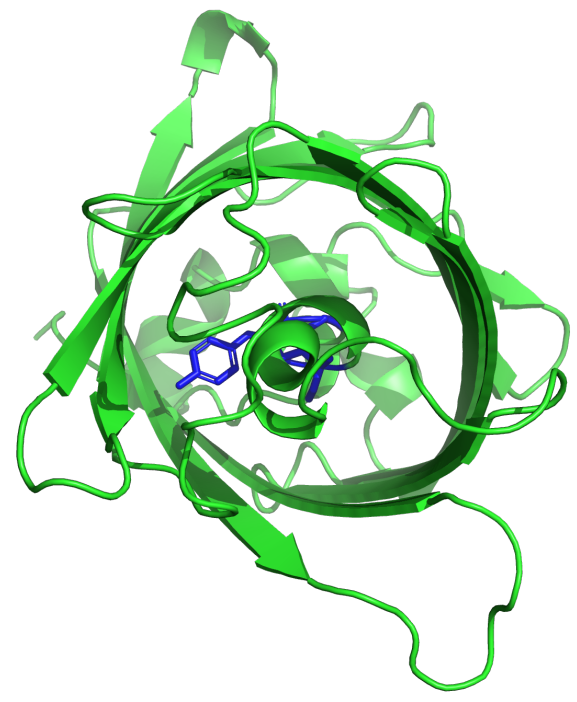

(B) Top view

FIGURE 1.5: Crystal structure of GFP in (A) side and (B) top view. The protein is shown in cartoon representation and the chromphore is shown in stick representation. The figure is based on PDB structure 1GFL [147].

case of fluorescent proteins, it is possible to genetically encode a fusion protein consisting of the molecule of interest coupled to the fluorescent protein via an amino acid linker. This recombinant fusion protein can be expressed transiently from a plasmid or it can be integrated stably into the genome typically by CRISPR/Cas- 9 genome editing [102]. In the following, only the use of fluorescent proteins as fluorescent labels will be discussed, as chemically synthesized fluorescent molecules were not used in this thesis.

\subsubsection{GFP-like fluorescent Proteins}

The first fluorescent protein was isolated from jellyfish Aequorea victoria in 1962 by Shimomura et al. [122]. Due to the green fluorescence, the protein was termed green fluorescent protein (GFP). GFP is a 238 amino acid protein with a molecular weight of $27 \mathrm{kDa}$ [97]. The crystal structure of GFP was solved in 1996 independently by Ormö et al. [92] and Yang et al. [147] and revealed a $\beta$-barrel structure consisting of $11 \beta$-sheets with a central $\alpha$-helix going through the barrel from top to bottom. Figure 1.5 shows the crystal structure of GFP. Its chromophore, 4(p-hydroxybenzylidene)-5-imidazolinone, is formed autocatalytically under oxygen atmosphere from amino acids serine 65 , tyrosine 66 and glycine 67 located in the central $\alpha$-helix of GFP $[104,154]$. The chemical structure of the chromophore is shown in figure 1.6. It was not before 1994, when GFP was used as fluorescent marker by Chalfie et al. [77].

Many GFP homologues have been discovered and engineered since then. They all share the typical $\beta$-barrel structure and form their respective chromophore autocatalitically. Due to their structural similarity, those proteins are categorized as GFPlike fluorescent proteins. Excitation and emission of GFP-like fluorescent proteins 
<smiles>[R]NC(CO)C1=N/C(=C\c2ccc(O)cc2)C(=O)N1CC([R])=O</smiles>

FIGURE 1.6: Structure of GFP chromophore. The chromophore is formed by amino acids serine 65 , tyrosine 66 and glycine 67 .

cover the entire visible spectrum. Figure 1.7 visualizes the spectral range of GFPlike fluorescent proteins. However, despite substantial efforts, it was not possible to expand excitation and emission of GFP-like fluorescent proteins to the near-infrared (NIR) spectral region between 650 to $900 \mathrm{~nm}$. This limitation was overcome by the employment of bacterial phytochrome based fluorescent proteins incorporating the external chromophore biliverdin and allowing excitation and emission in the NIR region.

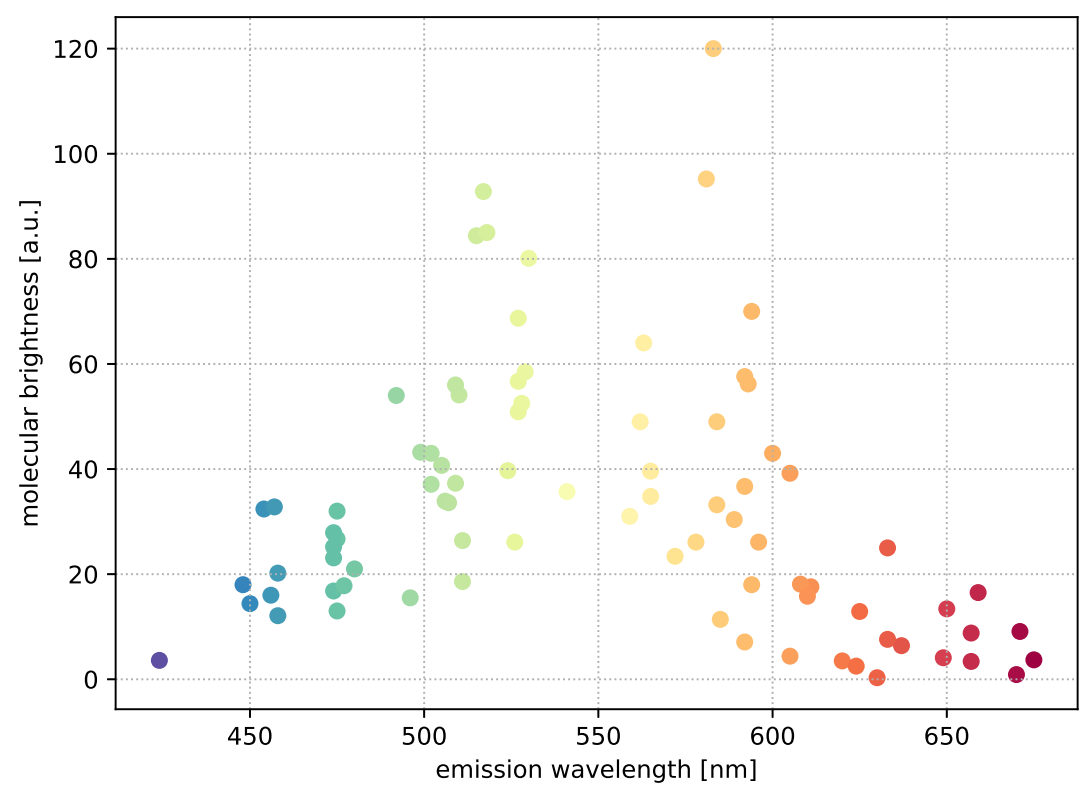

FIGURE 1.7: Correlation between molecular brightness and emission wavelength of GFP-like fluorescent proteins. Data points are colored in correspondence to the emission wavelength of the respective protein. The data were taken from the "Fluorescent Protein Database" [42].

\subsection{Phytochromes}

Besides GFP-like fluorescent proteins that form their chromophore autocatalytically, organisms have developed a wide collection of photoreceptors that gain their specific optical properties by binding small molecules as external chromophore. One representative of this class of photoreceptors is the family of phytochrome proteins that bind a linear tetrapyrrole as chromophore. Phytochromes have been found 
in plants [100], fungi [21], algae and bacteria [11] and play crucial roles in lightresponsive processes [66]. For example, Arabidopsis thaliana expresses five phytochrome isoforms that regulate photomorphogenesis [100, 124]. In bacteria, phytochrome proteins play important roles in intracellular signalling and regulation of gene expression [77].

Most members of the phytochrome family share a common modular structure, consisting of an N-terminal photosensory core domain (PCM) and a C-terminal regulatory domain [99]. The PCM module of prototypical phytochrome proteins consists of a PAS (Per-ARNT-Sim), a GAF (cGMP phosphodiesterase/adenylate cyclase/FhlA) and a PHY (phytochrome specific) domain. The tetrapyrrole chromophore is bound by and embedded in the PCM domain. Bacteria and fungi bind biliverdin IX $\alpha$ (BV) $[11,73,16]$ as their chromophore, while cyanobacteria and plants incorporate phycocyanobilin (PCB) [60] and phytochromobilin (PФB) [71], respectively.

Biosynthesis of linear tetrapyrroles is part of the heme metabolism [134, 43]. In a first step heme is degraded to BV by enzymatic oxidation catalysed by heme oxygenase [134]. In oxygenic photosynthetic organisms, BV is further metabolised to PCB and РФB by ferredoxin-dependent bilin reductase [43]. In contrast to oxygenic photosynthetic organisms, mammals metabolise BV to bilirubin IX $\alpha$ (BR) catalysed by NADPH-dependent biliverdin reductase $[78,117]$. The reverse reaction, the oxidation of BR to BV, is catalised by bilirubin oxidase [63]. In the human body, approximately $300 \mathrm{mg}$ BR are produced daily mainly due to degradation of red blood cells $[68,17]$. It is excreted from the body via bile and urine [135]. The level of BR in the blood is an indicator for several diseases, e.g. jaundice [12] or cholestasis [13]. Biliverdin and bilirubin are ubiquitously present in submicromolar concentrations in mammalina cells $[39,123,94]$. The tightly regulated balance between BV and BR is part of the protective system of the cell against oxidative stress [63].

Phytochromes can adapt two distinct stable states, a red light absorbing Pr state, and a far-red light absorbing Pfr state $[100,115]$. The states are interconvertible either by photoisomerization or by dark-conversion. Most phytochromes adapt the Pr state as ground state $[138,139,38]$. Only the so-called bathyphytochromes adapt the Pfr state as their ground state $[149,148]$. Crystal structures of bacterial phytochromes show a 5Zsyn, 10Zsyn, 15Zanti (ZZZssa) configuration of BV in the Pr state $[138,139]$. The same configuration was found for PCB in a crystal structure of Cph1 phytochrome from cyanobacterium Synechocystis 6803 [38]. Crystal structures of bathy-phytochromes in the Pfr state reveal a 5Zsyn, 10Zsyn, 15Eanti (ZZEssa) configuration $[149,148]$.

The spectral properties of phytochromes are governed by the tetrapyrrole chromophore they incorporate and its interaction with the surrounding protein scaffold. The absorption maxima of protein-bound PCB and РФB are located within the red spectral region. Only the absorption of protein-bound BV is located well within the NIR spectral region between 650 to $900 \mathrm{~nm}$ for most phytochrome proteins. For live-cell deep-tissue fluorescence microscopy, this spectral region is very beneficial. Absorbance of abundant molecules like water, haemoglobin and oxyhaemoglobin is minimal between 650 to $900 \mathrm{~nm}[120,142]$ as can be seen from figure 1.8. Additionally, phototoxicity, light scattering, abberations [14] and autofluorescence are reduced when imaging in the NIR spectral region as compared to imaging in the 
visible spectral region. Due to the unique properties of their external chromophore, bacterial phytochrome based fluorescent proteins are ideal candidates for engineering fluorescent probes for NIR fluorescent imaging.

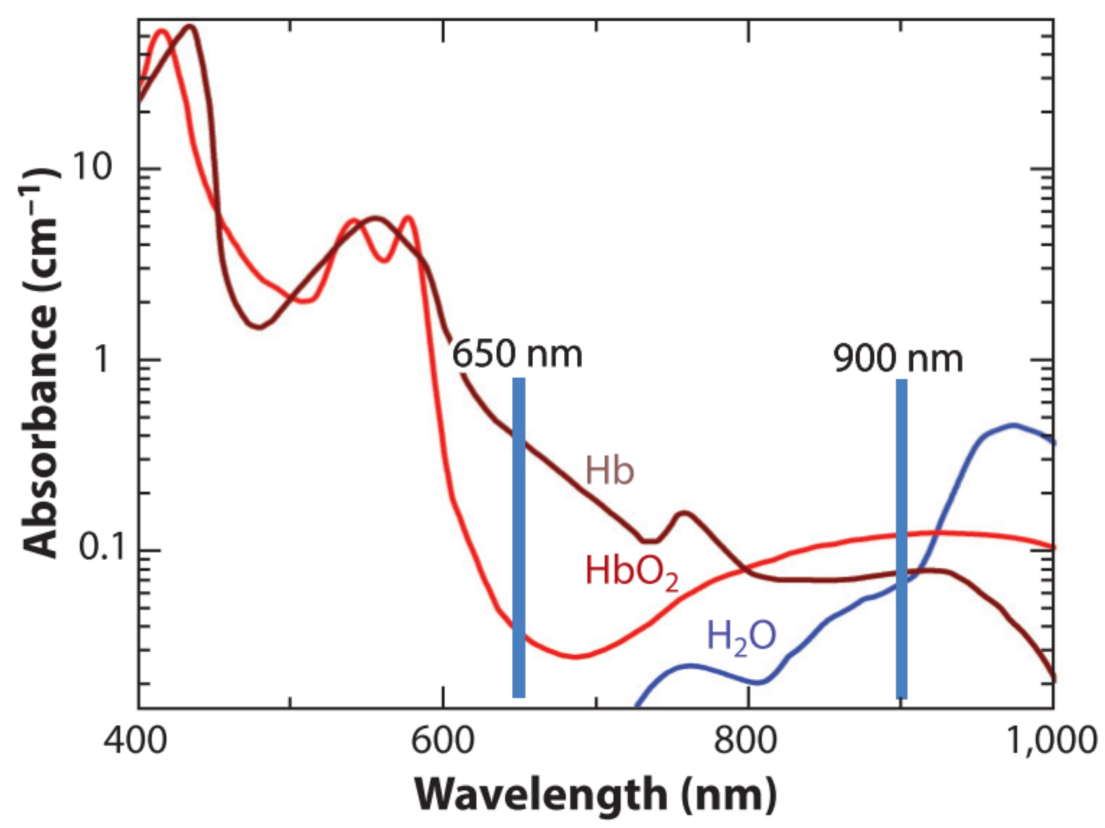

FIGURE 1.8: The absorbance of water, haemoglobin and oxyhaemoglobin in the visible and NIR spectral region. The figure was adopted and altered from Shscherbakova et al. [120]. Copyright 2015,

Annual Reviews.

\subsubsection{Bacterial Phytochromes}

In this work, the engineered bacterial phytochrome miRFP703 [119, 121] was used as a starting point for further improvement of fluorescence parameters. Therefore, in this subsection the structure, photocycle and properties of bacterial phytochromes are discussed in more detail.

The first crystal structure of a bacterial phytochrome was the chromophore binding domain, i.e. the PAS and the GAF domain, of Deinococcus radiodurans (DrCBD) obtained by Wagner et al. [138] in 2005. In 2008, Yang et al. [149] published a crystal structure of a bacterial phytochrome from Pseudomonas aeruginosa consisting of PCM and PHY domain. The structure can be seen in figure 1.9. The PAS domain consists of five antiparallel $\beta$-sheets (ordered 2-1-5-4-3) and three flanking $\alpha$-helices. Those structured elements are preceded by approximately 35 amino acids that do not adapt any defined secondary structure. Within those unstructured amino acids, a cysteine is located that covalently binds the chromophore BV via thioether linkage to the C3 atom of BV [73]. The PAS domain is followed by the GAF domain. The GAF domain is formed by six antiparallel $\beta$-sheets (ordered 9-10-11-6-7) and five $\alpha$-helices. The GAF domain forms the chromophore binding pocket.

PAS and GAF domain are connected via a ten amino acid linker and, more importantly, by a figure-of-eight knot structure first described by Wagner et al. [138] (see figure 1.10) based on the crystal structure of $\operatorname{DrCBD}$. This structural element is formed when the unstructured N-terminal region of the PAS domain pierces through 


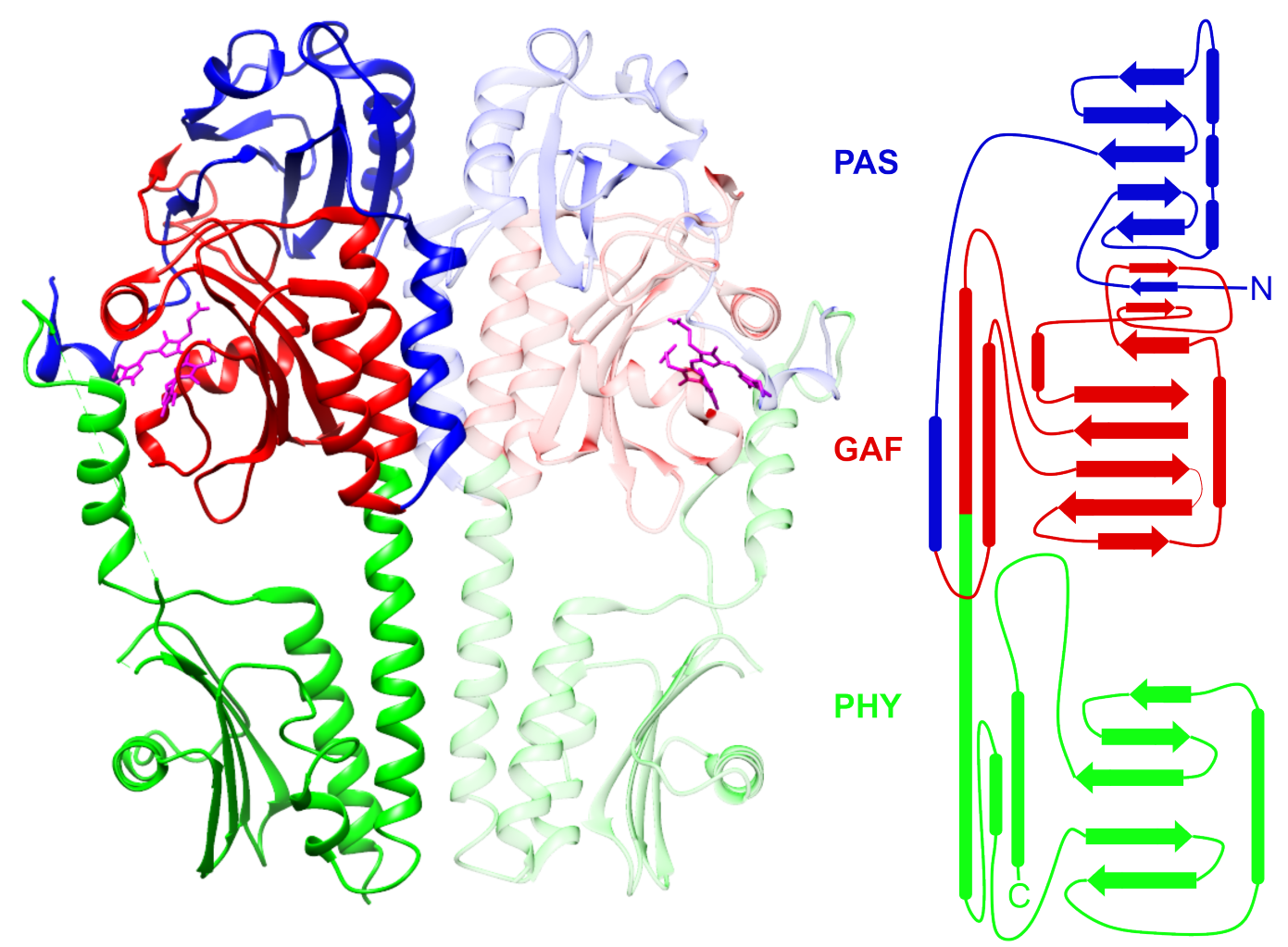

FIGURE 1.9: Left: Crystal structure of bacterial phytochrome dimer from $P$. aeruginosa (PDB ID: 3C2W [149]) with PAS domain (blue), GAF domain (red), PHY domain (green), and BV chromophore (magenta). Right: Schematic representation of bacterial phytochrome structure with PAS domain (blue), GAF domain (red), PHY domain (green).

a loop formed by two small $\alpha$-helices from the GAF domain. The knot is stabilized by a hydrophobic core. In the case of $\operatorname{DrCBD}$, Ile35 is making van der Waals contacts to Leu41, Val232, Leu234, Leu248 and Leu253 [138]. The function of this structural element is not entirely clear, but it was proposed that it is important for stabilizing the connection between PAS and GAF domain and helps positioning the cystein residue in the N-terminal region for efficient chromophore binding [138, 127]. The knot structure is very conserved in most phytochromes. Only phytochromes without a PAS domain do not contain this structural element [136]. The folding of proteins into knotted structures is very rare and was only observed for very few examples $[132,89,133,153]$.

The spectral properties of bacterial phytochromes are tuned by the interactions between the BV and its binding pocket [138]. The chromophore is embedded in a pocket formed by the GAF $\beta$-sheet and $\alpha$-helices 6 and 7 [138]. The pocket is shielded from solvent water via an extension of the PHY domain that reaches over the pocket [149]. Only a correct positioning of the BV chromophore in the binding pocket allows the formation of the thioether linkage [3]. A particularly important role for this positioning was assigned to the propionic-acid side chains of BV's B and $C$ rings [138]. Charged interactions, hydrogen bonds and hydrophobic interactions between highly conserved amino acids and the $\mathrm{B}$ and $\mathrm{C}$ rings were described 


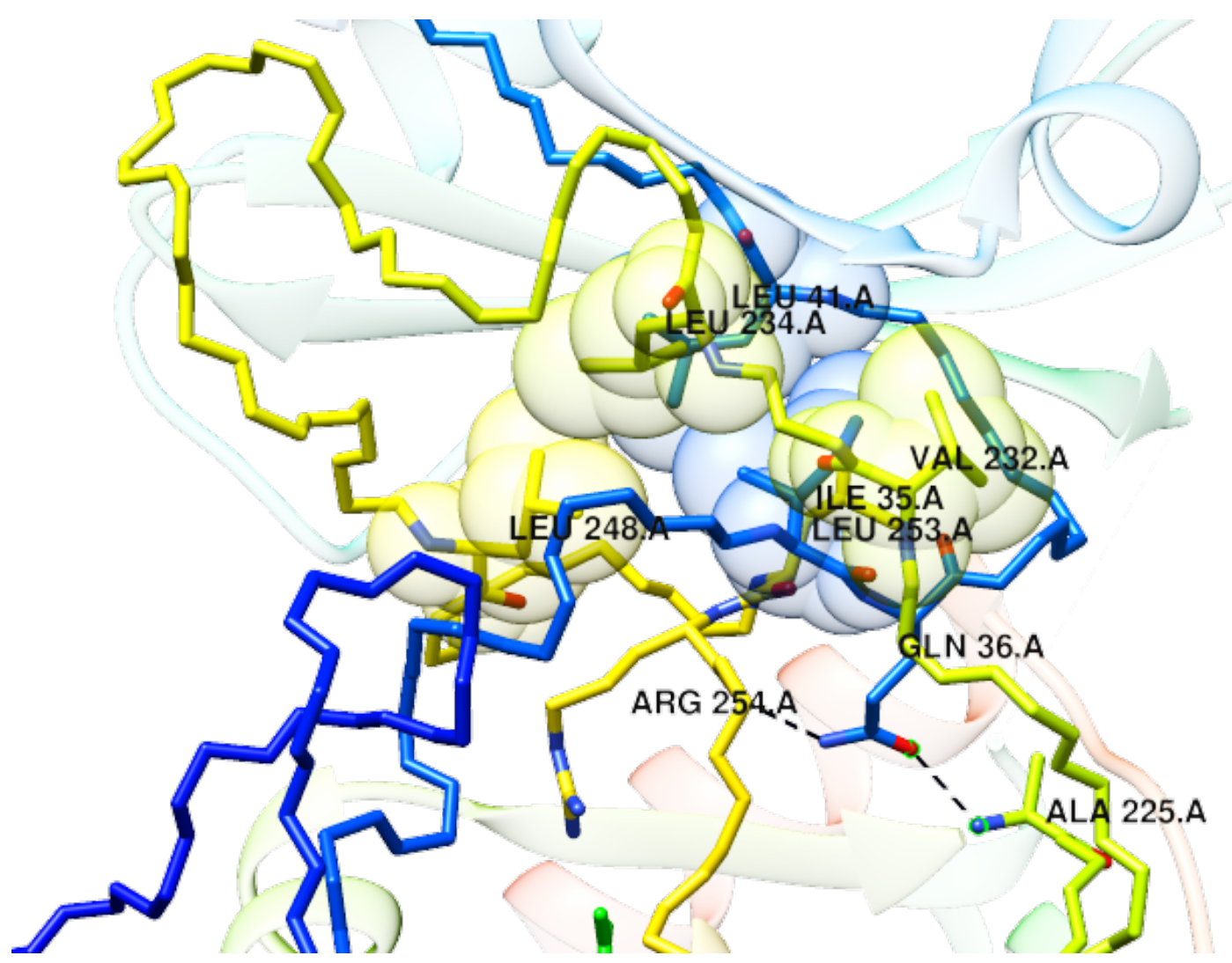

FIGURE 1.10: Figure-of-eight knot from $\operatorname{DrCBD}$. The protein scaffold is colored in rainbow color scheme (N-terminus, blue to $\mathrm{C}$ terminus, red; figure-of-eight knot: minimal backbone representation, rest: transparent cartoon representation). The amino acids that are located within the stabilizing hydrophobic core of the knot are additionally represented by their van der Waals radii. The BV chromophore is colored in magenta. The figure is based on PDB structure 1ZTU [138].

[138]. For $\operatorname{DrCBD}$, the carbonyl oxygens of BV's B ring are forming a salt bridge to Arg254 and hydrogen bonds to Tyr216 and Ser257. The $C$ rings oxygens are forming hydrogen bonds to His260, Ser272 and Ser274 (see figure 1.11). Another hydrogen bond is formed between the C ring proprionate and His 290 mediated by a water molecule [138]. Another water mediated network is formed between His 260 and the nitrogen atoms of BV's A, B and C rings [3] (see figure 1.12a). A highly conserved interaction between the protein scaffold and the BV chromophore takes place via the so called DIP motif (see figure 1.12b). For DrCBD, the DIP motif consists of amino acids Asp207, Ile208 and Pro209. It creates a kink in the polypeptide chain and allows residues access to the direct chromophore environment. This enables hydrophobic interactions between Ile208 and the C ring, Pro209 and the C10 carbon and Pro209 and the B ring. [138]. BV's D ring however only makes little interaction with the surrounding amino acids of the protein. The relative space around the D ring is necessary to allow photoisomerization of BV around the C15-C16 double bond [138].

In order to perform their natural functions, bacterial phytochromes can undergo a photoisomerization process between two stable conformations. The stable conformations are spectrally different and are called Pr (red absorbing) and Pfr (far-red 


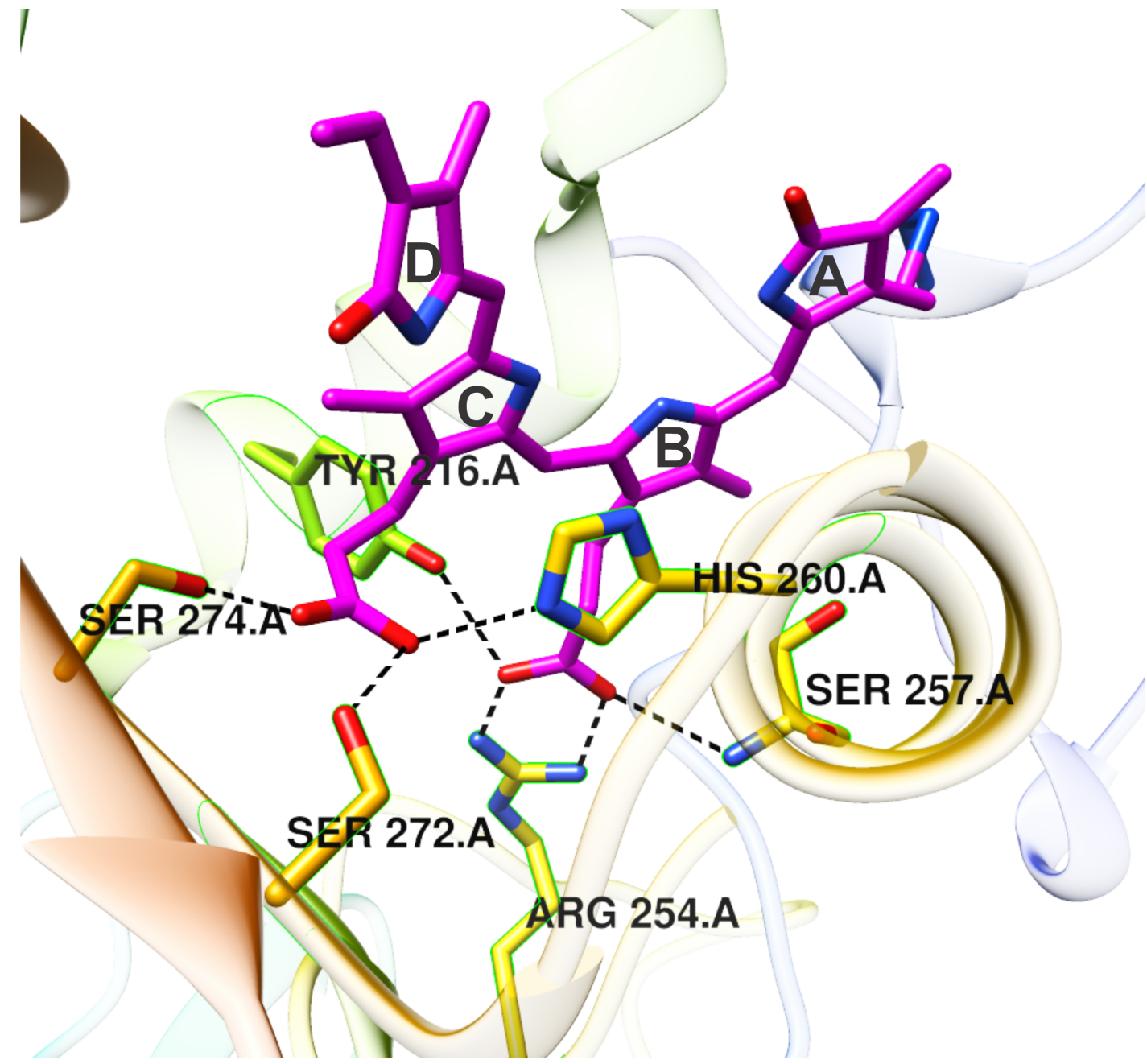

FIGURE 1.11: Interaction between protein scaffold and BV carbonyls from $\operatorname{DrCBD}$. The protein scaffold is shown in cartoon representation and colored in rainbow color scheme (N-terminus, blue to $\mathrm{C}$ terminus, red). The amino acid side chains that interact with the BV carbonyls are shown in stick representations. The BV chromophore is colored in magenta. Interactions (hydrogen bonds and salt bridges) are indicated by dotted black lines. The figure is based on PDB structure 1ZTU [138].

absorbing) state. The process can be divided into a fast cis-trans photoisomerization of the BV chromophore around the $\mathrm{C} 15-\mathrm{C} 16$ double bond and a slower subsequent restructuring of the protein scaffold [131, 140].

For the photoconversion from the Pr to the Pfr state, different intermediates have been identified [140, 143, 41] (see figure 1.13). Upon absorption of a red photon the chromophore in the Pr state can rotate around the C15-C16 double bond and form a metastable Meta-R state within ps. From Meta-R state the protein relaxes thermally to Meta- $\mathrm{R}_{a}$ and further to Meta- $\mathrm{R}_{c}$ state within $\mu$ s to $\mathrm{ms}$, respectively. The formation of Meta- $\mathrm{R}_{c}$ is accompanied by deprotonation of the chromophore. Subsequently, Pfr state is formed from Meta- $R_{c}$ within ms by reprotonation of the chromophore. The reverse photoconversion from the Pfr state to the Pr state is triggered by the absorption of a far-red photon and involves different intermediates. The first intermediate, Lumi-F, is formed within ps and is characterized by the trans-cis isomerization of the 


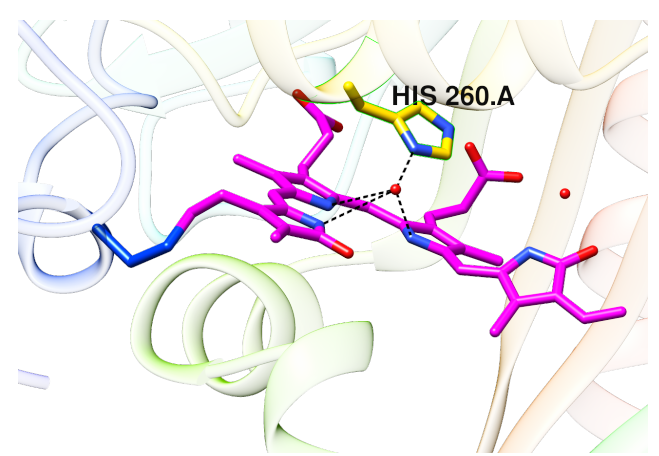

(A) Pyrrole water

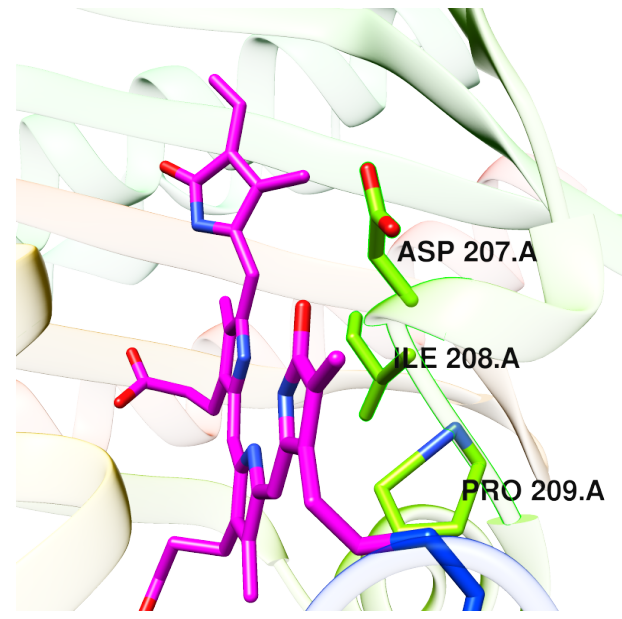

(B) DIP motif

FIGURE 1.12: (A) DIP motif and (B) pyrrole water from $\operatorname{DrCBD}$. The protein scaffold is shown in cartoon representation and colored in rainbow color scheme (N-terminus, blue to $\mathrm{C}$-terminus, red). The amino acid side chains that interact with the BV carbonyls are shwon in stick representations. The BV chromophore is colored in magenta. Interactions (hydrogen bonds) are indicated by dotted black lines.

The figure is based on PDB structure 1ZTU [138].

chromophore. Lumi-F relaxes to Meta- $\mathrm{F}_{a}$ within $\mathrm{ms}$ and further Meta- $\mathrm{F}_{b}$ within $\mu \mathrm{s}$ and Pr within ms. In contrast to the photoconversion from Pr to Pfr, the reverse photoconversion does not involve a proton transfer. For most bacterial phytochromes, the Pfr state is not thermally stable and can relax to the Pr state via dark conversion. The rate of dark conversion can be influenced by external factors, e.g. $\mathrm{pH}$, ionic strength and interaction with other proteins [108, 129]. It was proposed that dark conversion is essential for the natural functionality of phytochromes [36].

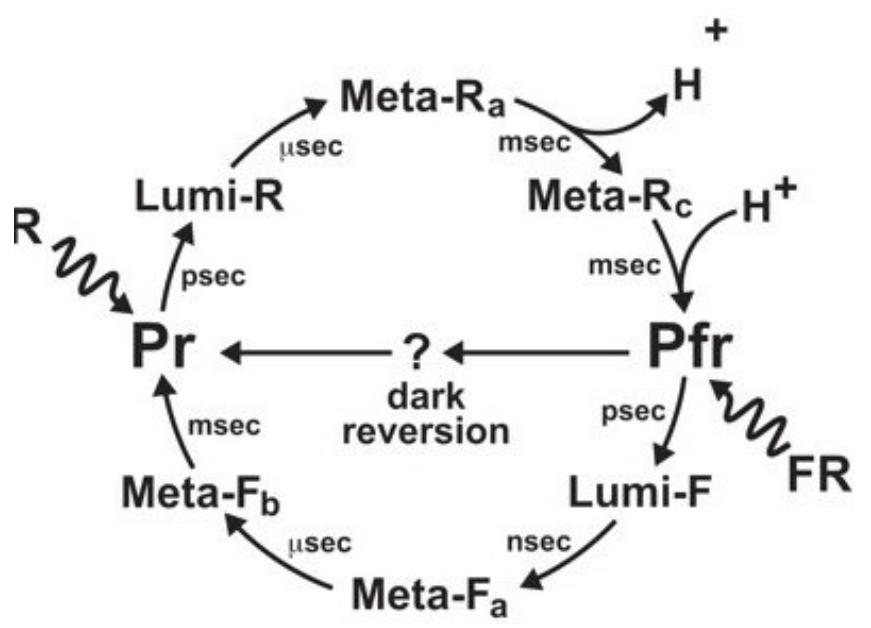

FIGURE 1.13: Schematic depiction of phytochrome photo cycle. The figure was adapted from Wagner et al. [140]. Copyright 2008, the American Society for Biochemistry and Molecular Biology. 
Following the photoconversion of the chromophore, structural changes of the surrounding protein scaffold take place. These changes are altering the proteinchromophore and protein-protein interactions. The extension of the PHY domain that covers the BV binding pocket in the Pr state undergoes extensive changes in secondary structure upon cis-trans isomerization of the chromophore [131]. Crystal structures from Takala et al. of D. radiodurans bacterial phytochrome in the Pr (PDB ID:4O0P) and Pfr (PDB ID:4O01) state show a change from an antiparallel $\beta$-sheet in the Pr state to an $\alpha$-helical structure in the Pfr state. With this, interactions between the PHY extension and the GAF domain are interrupted. The exact mechanism of signal transduction to the effector domain is unknown and different models have been proposed [150,131, 130]. However, the models agree on a reduced activity of the effector domain in the Pfr state as compared to the Pr state [131, 130, 150, 87].

\subsubsection{Phytochrome based Fluorescent Proteins}

In 2009, Shu et al. [123] published the first engineered bacterial phytochrome based NIR fluorescent protein termed IFP1.4. This fluorescent protein was derived from D. radiodurans and was truncated to CBD only. Since then, a variety of engineered NIR fluorescent proteins was published. A non-exhaustive selection of published proteins can be found in table 1.1.

\begin{tabular}{l|l|c|c|c} 
Name & Organism & $\begin{array}{c}\text { Excitation/ } \\
\text { Emission [nm] }\end{array}$ & $\begin{array}{c}\text { Quantum } \\
\text { Yield [\%] }\end{array}$ & Reference \\
\hline IFP1.4 & D. radiodurans & $684 / 708$ & 7.7 & {$[123]$} \\
IFP2.0 & D. radiodurans & $690 / 711$ & 8.1 & {$[152]$} \\
iRFP670 & R. palustris & $643 / 670$ & 12.2 & {$[118]$} \\
iRFP682 & R. palustris & $663 / 682$ & 11.1 & {$[118]$} \\
iRFP702 & R. palustris & $673 / 702$ & 8.2 & {$[118]$} \\
iRFP720 & R. palustris & $702 / 720$ & 6.0 & {$[118]$} \\
miRFP670 & R. palustris & $642 / 670$ & 14.0 & {$[119]$} \\
miRFP703 & R. palustris & $674 / 703$ & 8.6 & {$[119]$} \\
miRFP709 & R. palustris & $683 / 709$ & 5.4 & {$[119]$} \\
miRFP670nano & N. punctiforme & $645 / 670$ & 10.8 & {$[90]$} \\
emiRFP670 & R. palustris & $642 / 670$ & 14.0 & {$[80]$} \\
emiRFP703 & R. palustris & $674 / 703$ & 8.6 & {$[80]$} \\
mIFP & Bradyrhizobium & $683 / 704$ & 8.4 & {$[151]$} \\
smURFP & T. erythraeum & $642 / 670$ & 18 & {$[109]$} \\
SNIFP & D. radiodurans & $687 / 720$ & 2.2 & {$[65,64]$} \\
mRhubarb713 & R. palustris & $690 / 713$ & 7.6 & {$[110]$} \\
GAF-FP & R. palustris & $653 / 670$ & 7.3 & {$[111]$} \\
BDFP1.5 & Chr. thermalis & $688 / 711$ & 5.6 & {$[30]$}
\end{tabular}

TABLE 1.1: Engineered NIR fluorescent proteins

Most of the engineered fluorescent proteins were derived from truncated bacterial phytochromes and consist only of the CBD. For classical bacterial phytochromes that bind the BV chromophore via a cysteine residue in the PAS domain and embed the chromophore in a pocket formed mainly by the GAF domain, the CBD, consisting of PAS and GAF domain, is the minimal protein scaffold to covalently incorporate BV. A $19 \mathrm{kDa}$ fluorescent protein, termed GAF-FP, consisting only of the GAF domain was engineered by Rumyantsev et al. [111]. This was achieved by removing 


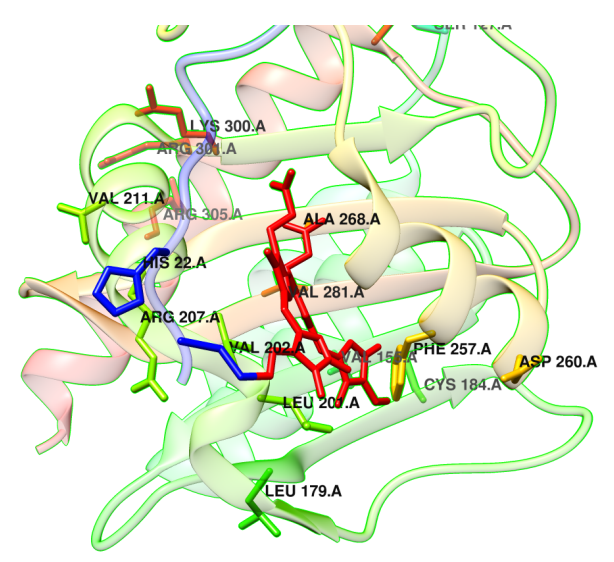

(A) Chromophore binding pocket

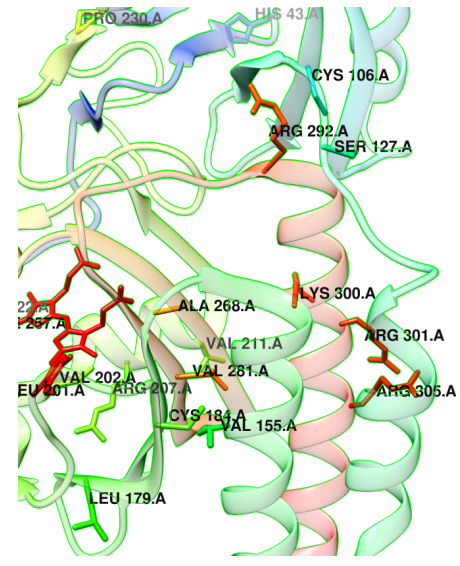

(B) Dimerization interface

FIGURE 1.14: Crystal structure of miRFP703. (A) Chromophore binding pocket and (B) dimerization interface of miRFP703. The protein scaffold is shown in cartoon representation and colored in rainbow color scheme (N-terminus, blue to C-terminus, red). The chromophore is colored in red. Mutations introduced to the protein as compared to the wild type sequence are shown in stick representation and labeled. The figure is based on PDB structure 5VIK [119].

the cysteine residue from the PAS domain and introducing it into the GAF domain to enable a chromophore binding similar to cyanobacteriochromes [73, 40]. However, the alternative binding of BV via a cysteine in the GAF domain causes a major spectral blue shift, resulting into absorption and emission of GAF-FP outside the desired NIR spectral window. Proteins like miRFP670 [119] and emiRFP670 [80] have two cysteine residues, one in the PAS and the other in the GAF domain. Studies showed that BV can be bound via both cysteine residues at the same time. This drastically increases the fluorescence quantum yield at the expense of a spectral blue shift $[58,18]$.

The engineered fluorescent proteins smURFP [109] and BDFP [30] are not derived from bacterial phytochromes but from phycobiliproteins found in the light harvesting complexes of cyanobacteria and red algae. Naturally, those proteins do bind PCB instead of BV. However, it could be shown, that it is possible to increase the affinity for BV using directed evolution $[109,30]$. Nevertheless, the major disadvantage of phycobiliproteins engineered to bind BV remains the low BV affinity [121]. This makes addition of external BV necessary when using those proteins in vivo. Additionally, all phycobiliprotein based fluorescent proteins have a high dimerization tendency which limits their usefulness as fusion tags in fluorescence microscopy.

For this work, the engineered fluorescent protein miRFP703 [119] was of particular importance. The protein was used as a template for the directed evolution to increase the fluorescence lifetime and the fluorescence quantum yield. The protein was selected because it was shown to be truly monomeric and exhibits a high effective brightness in mammalian cells with excitation and emission within the NIR spectral region. The protein is based on the CBD of a bacterial phytochrome found in $R$. palustris. In order to repress the photoisomerization of the chromophore and thereby increase the fluorescence quantum yield, mutations D201L, I202V and Y257F were introduced $[119,4]$ (see figure 1.14a). The C-terminal $\alpha$-helix of the GAF domain was subjected to random mutagenesis to disrupt the dimerization interface 
[119] (see figure 1.14b).

To this point, available NIR fluorescent proteins suffer from at least one of two problems. Either they have a relatively low fluorescence quantum yield as compared to GFP-derived fluorescent proteins (e.g. miRFP703 [119], mIFP [151], SNIFP [65, 64], iRFP703 [118]) making it challenging to obtain bright fluorescence images with high signal to background ratio, or they exhibit a major blue shift (e.g. miRFP670 [119], emiRFP670 [80], miRFP670nano [90], smURFP [109]) rendering them useless for true NIR imaging with excitation and emission within the NIR spectral window between 650 to $900 \mathrm{~nm}$.

\subsection{Aim of the Study}

The utilization of engineered bacterial phytochrome variants opened the NIR spectral window for fluorescence microscopy. However, despite substantial efforts all available NIR fluorescent proteins absorbing and emitting beyond $650 \mathrm{~nm}$ suffer from a short fluorescence lifetime and a low fluorescence quantum yield, limiting their potential for fluorescence microscopy.

The goal of this work was the development of a bacterial phytochrome based NIR fluorescent protein with increased fluorescence lifetime and fluorescence quantum yield. In the first part of this work, an automated screening microscope was planned and build to screen for protein variants with increased fluorescence lifetime. Based on the correlation between the fluorescence lifetime and the fluorescence quantum yield it was hypothesized that protein variants with increased fluorescence lifetimes would also exhibit increased fluorescence quantum yields. The developed screening system was used in the second part of this thesis to increase the fluorescence lifetime of a template protein via consecutive rounds of diversification and screening. In the third part of this work, the improved protein variant was characterized biophysically. Afterwards its applicability as fusion tag in confocal and STED live-cell fluorescence microscopy was demonstrated. In the last part, fluorescence lifetime imaging was employed to perform two-color confocal and STED imaging entirely in the NIR spectral region beyond $650 \mathrm{~nm}$ for the first time. 



\section{Chapter 2}

\section{Material and Methods}

This chapter describes the methods used in this thesis. In order to keep the descriptions compact, cloning primers and buffer compositions are listed in appendix A and appendix B, respectively.

\subsection{Molecular Biology}

Different mutagenesis techniques were employed in this work. Standard ligation and Gibson cloning were used to exchange fluorescent proteins in plasmids. Site directed, multiple site and error prone mutagenesis were used to create diversified pools of protein variants for directed evolution of fluorescent proteins.

\subsubsection{Standard Cloning}

For standard cloning, the insert was amplified using polymerase chain reaction (PCR), digested using the respective restriction enzymes and ligated with the restricted vector backbone. To amplify the insert, approximately $250 \mathrm{ng}$ DNA was used in standard PCR buffer (see appendix B) together with $0.2 \mathrm{mM}$ of each dNTP (Thermo Fisher Scientific, Waltham, MA, USA), $0.3 \mu \mathrm{M}$ of each primer and $1 \mu \mathrm{l}$ Pfu polymerase (biotechrabbit, Henningsdorf, Germany) in a total volume of $50 \mu 1$. The reaction was prepared on ice. The PCR reaction was performed using a Biometra $T_{\text {one }}$ Thermocycler (Analytik Jena, Jena, Germany) following the protocol listed in table 2.1. After the PCR reaction $1 \mu \mathrm{l}$ Dpn1 (Thermo Fisher Scientific, Waltham, MA,

\begin{tabular}{|c|c|c|}
\hline $98^{\circ} \mathrm{C}$ & $300 \mathrm{~s}$ & \\
\hline $98^{\circ} \mathrm{C}$ & $60 s$ & \multirow{3}{*}{35 cycles } \\
\hline $55-65^{\circ} \mathrm{C}$ & $60 s$ & \\
\hline $72{ }^{\circ} \mathrm{C}$ & $60 \mathrm{~s} / 1 \mathrm{~kb}$ & \\
\hline $72^{\circ} \mathrm{C}$ & $600 \mathrm{~s}$ & \\
\hline $4^{\circ} \mathrm{C}$ & $\infty$ & \\
\hline
\end{tabular}

TABLE 2.1: PCR protocol

USA) was added to the reaction mix and incubation was performed for $2 \mathrm{~h}$ or over night at $37^{\circ} \mathrm{C}$. Subsequently, the PCR product was purified using the NucleoSpin ${ }^{\circledR}$ Gel and PCR Clean-up kit (Macherey-Nagel, Düren, Germany). The purified PCR product and the vector backbone were digested using the appropriate restriction enzymes (Thermo Fisher Scientific, Waltham, MA, USA) with the respective buffers (Thermo Fisher Scientific, Waltham, MA, USA) according to the manufacturers instructions. After restriction $1 \mu \mathrm{l}$ of CIP (New England Biolabs, Ipswich, MA, USA) was added to the restricted vector backbone for dephosphorylation. Subsequently, 
PCR product and vector backbone were purified using agarose gel electrophoresis. For this, agarose (BioBudget Technologies, Krefeld, Germany) was dissolved in $1 \mathrm{x}$ TAE buffer at a concentration of $1.2 \%(\mathrm{w} / \mathrm{v})$. Gels were casted in BlueMarine ${ }^{\mathrm{TM}}$ 100 gel chambers (Serva Electrophoresis, Heidelberg, Germany). The electrophoresis was performed for approximately $1 \mathrm{~h}$ at a constant voltage of $80 \mathrm{~V}$ delivered by a voltage source (VWR, Radnor, PA, USA). Together with the samples, GeneRuler ${ }^{\mathrm{TM}}$ $1 \mathrm{~kb}$ DNA ladder (Thermo Fisher Scientific, Waltham, MA, USA) was loaded for reference. After electrophoresis gels were stained in an ethidium bromide solution for approximately $20 \mathrm{~min}$. Gels were analyzed under UV light and desired bands were isolated using a scalpel. The DNA was isolated from the gel slices using the NucleoSpin ${ }^{\circledR}$ Gel and PCR Clean-up kit according to the manufacturers instructions. Ligation of insert and vector backbone was carried out in 1x T4 DNA ligase buffer (Thermo Fisher Scientific, Waltham, MA, USA) with $2 \mu$ purified vector backbone, $6 \mu \mathrm{l}$ insert and $0.5 \mu \mathrm{l} \mathrm{T4}$ DNA ligase (Thermo Fisher Scientific, Waltham, MA, USA) at a total volume of $20 \mu \mathrm{l}$. Incubation was performed either over night at $4{ }^{\circ} \mathrm{C}$ or for $2 \mathrm{~h}$ at room temperature. Ligations were stopped at $65^{\circ} \mathrm{C}$ for $15 \mathrm{~min}$. Before transformation of $5 \mu$ into electrocompetent $E$. coli cells, the ligation reaction was dialysed for $1 \mathrm{~h}$ using $0.025 \mu \mathrm{m}$ MCE MF-Millipore ${ }^{\mathrm{TM}}$ membranes (Merck, Darmstadt, Germany). The transformed cells were plated on agar plates with the appropriate antibiotics and incubated over night at $37^{\circ} \mathrm{C}$. Resulting colonies were sent for sequencing to verify the successful cloning.

\subsubsection{Gibson Cloning}

For Gibson cloning, the insert was amplified using PCR as described in chapter 2.1.1. The vector backbone was either prepared by digestion with appropriate restriction enzymes (Thermo Fisher Scientific, Waltham, MA, USA) following the manufacturers instructions or via PCR. Before the Gibson reaction the products were purified by agarose gel electrophoresis. The Gibson reaction was performed using the Gibson Assembly ${ }^{\circledR}$ kit (New England Biolabs, Ipswich, MA, USA) in agreement with the manufacturers protocol. Success of the cloning procedure was verified by sequencing the resulting E. coli colonies.

\subsubsection{Site Directed Mutagenesis}

To change up to 6 successive base pairs, site directed mutagenesis was performed. To this end, approximately $250 \mathrm{ng}$ template DNA, $0.2 \mathrm{mM}$ of each dNTP (Thermo Fisher Scientific, Waltham, MA, Germany), $0.3 \mu \mathrm{M}$ of each primer and $1 \mu \mathrm{Pfu}$ polymerase (biotechrabbit, Henningsdorf, Germany) were added to 1x standard PCR buffer (see appendix B) to a total volume of $50 \mu \mathrm{l}$. PCR was performed according to the protocol shown in table 2.1. After the PCR reaction, the template DNA was digested by adding $1 \mu \mathrm{l}$ Dpn1 (Thermo Fisher Scientific, Waltham, MA, USA) to the reaction mix and incubating for either $2 \mathrm{~h}$ or over night at $37^{\circ} \mathrm{C}$. Before transformation into electrocompetent $E$. coli cells the samples were dialysed for $1 \mathrm{~h}$ using $0.025 \mu \mathrm{m} \mathrm{MCE}$ MF-Millipore ${ }^{\mathrm{TM}}$ membranes (Merck, Darmstadt, Germany).

\subsubsection{Multiple Site Mutagenesis}

For mutating more than one position in a DNA template, multiple site mutagenesis [114] was performed. To this end, approximately $500 \mathrm{ng}$ template DNA, $0.28 \mu \mathrm{M}$ foward primer with 5'-phosphorylation, $0.2 \mathrm{mM}$ of each dNTP, $1 \mu \mathrm{Pfu}$ polymerase 
Protocol 1

$\left.\begin{array}{ll}65^{\circ} \mathrm{C} & 300 \mathrm{~s} \\ \hline 95^{\circ} \mathrm{C} & 120 \mathrm{~s} \\ \hline 95^{\circ} \mathrm{C} & 30 \mathrm{~s} \\ 55^{\circ} \mathrm{C} & 30 \mathrm{~s} \\ 65^{\circ} \mathrm{C} & 600 \mathrm{~s}\end{array}\right\} 18$ cycles

Protocol 2

\begin{tabular}{ccc}
$37^{\circ} \mathrm{C}$ & $1 \mathrm{~h}$ & \\
\hline $95^{\circ} \mathrm{C}$ & $30 \mathrm{~s}$ & \\
\hline $95^{\circ} \mathrm{C}$ & $30 \mathrm{~s}$ & \\
$55^{\circ} \mathrm{C}$ & $60 \mathrm{~s}$ \\
$70^{\circ} \mathrm{C}$ & $600 \mathrm{~s}$ & \\
\hline $4^{\circ} \mathrm{C}$ & $\infty$ &
\end{tabular}

TABLE 2.2: Multiple site mutagenesis cycler protocol

and $0.5 \mu \mathrm{l}$ Taq DNA ligase were added to $0.5 \mathrm{x}$ standard PCR buffer and 0.5x Tag ligase buffer with a total volume of $50 \mu \mathrm{l}$. The first DNA strand was amplified via protocol 1 in table 2.2. Subsequently, $1 \mu \mathrm{Lpn} 1$ and $1.5 \mu \mathrm{l}$ reverse primer $(10 \mu \mathrm{M})$ were added to the reaction mix before running protocol 2 from table 2.2. After completion of the protocol, samples were dialyzed for $1 \mathrm{~h}$ using $0.025 \mu \mathrm{m}$ MCE MF-Millipore ${ }^{\mathrm{TM}}$ membranes (Merck, Darmstadt, Germany) and transformed into electrocompetent E. coli cells.

\subsubsection{Error Prone Mutagenesis}

In order to introduce random mutations into a DNA sequence, error prone mutagenesis was performed. Approximately $1 \mu \mathrm{g}$ of template DNA, $10 \mu \mathrm{l}$ error-prone dNTPmix, $1 \mu \mathrm{M}$ of each primer, $2 \mu \mathrm{l} \mathrm{GoTaq}{ }^{\circledR}$ DNA polymerase (Promega, Madison, WI, USA) and $0.2-0.3 \mathrm{mM} \mathrm{MnCl} 2$ were added to $1 \mathrm{x}$ error prone buffer to a total volume of $100 \mu \mathrm{l}$. The desired error rate was adjusted via the concentration of $\mathrm{MnCl}_{2}$. The $100 \mu \mathrm{l}$ reaction was split up into 4 separate tubes before performing the PCR protocol described in table 2.3. After the PCR reaction the separate tubes were combined for

\begin{tabular}{|c|c|c|}
\hline $95^{\circ} \mathrm{C}$ & $60 \mathrm{~s}$ & \\
\hline $95^{\circ} \mathrm{C}$ & $30 s$ & \\
\hline $\begin{array}{c}55-65^{\circ} \mathrm{C} \\
68^{\circ} \mathrm{C}\end{array}$ & $\begin{array}{c}45 \mathrm{~s} \\
60 \mathrm{~s} / 1 \mathrm{~kb}\end{array}$ & 30 cycles \\
\hline $68^{\circ} \mathrm{C}$ & $600 \mathrm{~s}$ & \\
\hline $4^{\circ} \mathrm{C}$ & $\infty$ & \\
\hline
\end{tabular}

TABLE 2.3: Error prone mutagenesis PCR protocol

further steps. The amplified PCR product and the vector backbone were purified, restricted with the appropriate restriction enzymes and ligated as described in chapter 2.1.1. In the end, the ligated product was transformed into E. coli for screening.

\subsection{Expression Systems}

In this work, different expression systems were used for specific experiments. For cloning, screening and protein expression and purification, bacterial expression systems were used. For fluorescence imaging and flow cytometry experiments, transient or stable mammalian expression systems were employed. 


\subsubsection{Bacterial Strains}

Different specialized bacterial E. coli strains were used for cloning, screening and recombinant protein expression. For cloning experiments, either E. coli TOP10 (One Shot $^{\mathrm{TM}}$ TOP10 Electrocomp ${ }^{\mathrm{TM}}$ E. coli, Thermo Fisher Scientific, Waltham, MA, USA) or DH5 $\alpha$ (Invitrogen, Carlsbad, CA, USA) cells were used. In protein expression experiments, E. coli BL21AI cells (BL21-AI ${ }^{\mathrm{TM}}$ One Shot ${ }^{\mathrm{TM}}$ Chemically Competent $E$. coli, Thermo Fisher Scientific, Waltham, MA, USA) were utilized.

\subsubsection{Cloning of Plasmids for Bacterial Expression}

For all experiments performed in E. coli cells the pBAD expression system (Thermo Fisher Scientific, Waltham, MA, USA) was used. More specifically the vector backbone of pBAD-smURFP-RBS-HO1 was utilized. It was a gift from Erik Rodriguez (Addgene \#80341 [109]). Additionally to the fluorescent protein, the vector encodes for the expression of heme oxygenase 1 (HO1) from the organsim Synechocystis. HO1 catalyzes the enzymatic oxidation of heme to biliverdin. Biliverdin is the external chromophore utilized in bacterial phytochrome proteins. Both, the expression of the fluorescent protein and $\mathrm{HO} 1$ is under the control of the araBAD promoter, that can be induced by arabinose. For protein purification a 6xHis-tag was added N-terminally to the fluorescent protein. For cloning of protein variants into this vector the primers 7819 and 7822 were used for PCR amplification of the insert sequence. Subsequently, the amplified insert was digested with BgIII and EcoRI and the vector was digested with BamHI and EcoRI. Thereafter, insert and vector backbone were ligated.

\subsubsection{Mammalian Cell Lines}

For transient expression in mammalian cells, HeLa cells were used. CRISPR/Cas-9 genome edited cell lines and stable Bxb1 landing pad cell lines were established with either HeLa or U2OS cells.

\subsubsection{Cloning of Plasmids for Transient Mammalian Expression}

For this work, fusion constructs of V410 with $\beta$-actin (actin), centromere protein C1 (CenpC1), histone 2B (H2B), peroxisomal targeting sequence (peroxi), cytokeratin18 (keratin), microtubule associated protein 2 (Map2), nuclear pore complex protein 50 (Nup50), vimentin, mito targeting sequence (mito) and translocase of outer mitochondrial membrane 20 (tomm20) were produced. All constructs were produced using the standard ligation cloning protocol as described in chapter 2.1.1. All primers and restriction enzymes used for cloning the constructs are summarized in table 2.4. The primer sequences can be found in table A.1.

To target $\beta$-actin, the coding sequence of $\beta$-actin was amplified from pTagFP635actin (Evrogen, Moscow, Russia) via PCR using the primers 4573 and 4574 . Subsequently, the coding sequence of human $\alpha$-tubulin in pEGFP-Tub (BD, Biosciences Clonetech, Franklin Lakes, NJ, USA) was exchanged for $\beta$-actin via BamHI and XhoI. To produce the final fusion construct of $\beta$-actin with V410, EGFP was exchanged via NheI and BglII for V410 amplified with primers 9561 and 9562.

The fusion construct of V410 with the mitochondrial targeting sequence was produced by exchanging DsRed in pDsRed1-Mito (BD Biosciences Clontech, Franklin Lakes, NJ, USA) via AgeI and NotI with the PCR product of V410 amplified with the 


\begin{tabular}{c|l|l|l|l}
$\#$ & Construct & Primers & Enzymes & Figure \\
\hline P2696 & V410-Map2 & $9596 / 9595$ & BglII/NheI & $3.11,3.12,3.20$ \\
P2697 & V410-Nup50 & $8911 / 8912$ & BglII/NheI & $3.11,3.17$ \\
P2698 & vimentin-V410 & $8229 / 8159$ & AgeI/NotI & 3.11 \\
P3227 & V410-CenpC1 & $9561 / 9562$ & NheI/XhoI & 3.11 \\
P3229 & V410-peroxi & $9561 / 9562$ & NheI/BglII & 3.11 \\
P3230 & V410-H2B & $9561 / 9562$ & NheI/BglII & 3.11 \\
P3231 & Mito-V410 & $9563 / 9564$ & AgeI/NotI & 3.11 \\
P3241 & cytokerain-18-V410 & $8227 / 8159$ & KpnI/NotI & $3.11,3.18$ \\
P3242 & V410- $\beta$-actin & $9561 / 9562$ & NheI/BglI & 3.11 \\
P3279 & V410-tomm20 & $9687 / 9688$ & AgeI/Not1 & $3.21,3.23,3.24$ \\
& & & & D.4, D.5 \\
P3319 & V410-P2A-mEGFP & $9788 / 9789$ & AgeI/EcoRI & 3.10, E.2 \\
P3320 & miRFP703-P2A-mEGFP & $9788 / 9789$ & AgeI/EcoRI & 3.10, E.2
\end{tabular}

TABLE 2.4: Summary mammalian constructs for transient expression

primers 9563 and 9564 .

The outer mitochondrial membrane was targeted with a tomm20 fusion construct. To this end, the coding sequence of tomm 20 was amplified via PCR from pDONR223-tomm 20 [72] using the primers 5570 and 5571. The PCR product was swapped via AgeI and NheI for the Mito targeting sequence in pDsRed1-Mito (BD Biosciences Clontech, Franklin Lakes, NJ, USA). DSRed was subsequently exchanged via AgeI and Not1 for the V410 coding sequence amplified with the primers 9687 and 9688.

In the case of keratin, TagRFP from pTagRFP-Keratin18 was swapped via KpnI and NotI for the coding sequence of V410 amplified with the primers 8227 and 8159 .

For CenpC1, pEGFP-CENP C [65] and the coding sequence of V410 amplified with the primers 9561 and 9562 were digested with NheI and XhoI. Subsequently, vector backbone and insert were ligated.

To target Map2, EGFP was exchanged via BglII and NheI in pEGFP-Map2 [65] for the PCR product of V410 amplified with the primers 9596 and 9595.

To produce a fusion construct of V410 with H2B, the coding sequence of V410 was amplified with primers 9561 and 9562. Subsequently, EGFP was swapped for the PCR product via NheI and BglII in pEGFP-Hist1H2Bn [65].

For Nup50, V410 was amplified using the primers 8911 and 8912. The PCR product and the plasmid mEmerald-Nup50-C-10 (Addgene \#54209) were ligated after digestion with BglII and NheI.

The fusion construct of the peroxisomal targeting sequence and V410 was constructed by replacing EGFP in pEGFP-PTS [65] via NheI and BglII with the coding sequence of V410 amplified with the primers 9561 and 9562.

To produce a fusion construct between vimentin and V410, the fluorescent protein mKate2 in pmKate2-vimentin (Evrogen, Moscow, Russia) was swapped via 
AgeI and NotI against the coding sequence of V410 amplified with the primers 8229 and 8159.

To compare the brightness of NIR FPs in mammalian cells, a plasmid was constructed that expressed the respective NIR fluorescent protein together with mEFGP as a NIR-FP-P2A-mEGFP fusion protein. Both proteins are encoded by a single mRNA and are connected via self-cleaving P2A sequence to ensure equal amounts of NIR FP and mEGFP. To this end, TagRFP was exchanged via AgeI and NotI in pTagRFP-N (Evrogen, Moscow, Russia) for the coding sequence of mEGFP amplified with the primers 1836 and 1837. Subsequently, the desired NIR FP was amplified using the primers 9788 and 9789 and inserted into the multiple cloning site of the vector via AgeI and EcoRI.

\subsubsection{Cloning of Plasmids for Stable Mammalian Cell Lines}

For this work, several stable mammalian cell lines were established. The plasmids were cloned using the standard ligation cloning protocol as described in chapter 2.1.1. All primers and restriction enzymes used for cloning the constructs are summarized in table 2.5.

\begin{tabular}{c|l|l|l|l}
$\#$ & Construct & Primers & Enzymes & Figure \\
\hline P2432 & $\begin{array}{l}\text { cytokeratin-18- } \\
\text { miRFP703-P2A-mEGFP }\end{array}$ & $8348 / 8352$ & HindIII/EcoRI & $3.21,3.23,3.24$, \\
P2690 & $\begin{array}{l}\text { cytokeratin-18- } \\
\text { V410-P2A-mEGFP }\end{array}$ & $8348 / 8352$ & HindIII/EcoRI & 3.20 \\
P2691 & $\begin{array}{l}\text { Vimentin-V410 } \\
\text { viment }\end{array}$ & $8357 / 8358$ & NcoI/NotI & 3.16
\end{tabular}

TABLE 2.5: Summary mammalian constructs for stable integration

\subsection{Cell Culture}

For expression of fusion constructs and measurement of fluorescence brightness in mammalian cells, either transiently transfected mammalian cells, CRISPR/Cas9 genome edited mammalian cells or stable Bxb1 landing pad cells were used.

The mammalian cells were cultured in T25 culture flasks (Sarstedt, Nümbrecht, Germany) using Dulbecco's Modified Eagle Medium (DMEM) with $4.5 \mathrm{~g} / \mathrm{L}$ glucose GlutaMax $^{\mathrm{TM}}, 1 \mathrm{mM}$ sodium pyruvate (Thermo Fisher Scientific, Waltham, MA,USA) and $10 \%(\mathrm{v} / \mathrm{v})$ fetal bovine serum (FBS) superior (Merck, Darmstadt, Germany). Bacterial contamination was suppressed by addition of $100 \mu \mathrm{g} / \mathrm{ml}$ streptomycin and $100 \mathrm{U} / \mathrm{ml}$ penicillin to the culture medium. The cells were grown in $\mathrm{CO}_{2}$ incubator at $37^{\circ} \mathrm{C}, 5 \% \mathrm{CO}_{2}$ and $90 \%$ humidity. Before the cells reached confluency, they were splitted into a new flask. To split the cells, DMEM was removed and the cells were washed twice in phosphate buffered saline (PBS), before addition of an appropriate volume of trypsin/EDTA (Merck, Damrstadt, Germany) and incubation for 10 to $15 \mathrm{~min}$ at $37^{\circ} \mathrm{C}$. Trypsination was stopped by addition of DMEM and cells were transferred in a new flask.

For long time storage, mammalian cells were frozen under liquid nitrogen conditions. To this end, cells were proliferated in T25 culture flasks. When confluency was 
reached, cells were washed twice in PBS and detached from the flask with trypsin. After the detachment was stopped by addition of DMEM, the cells were transferred to $15 \mathrm{ml}$ tubes (Sarstedt, Numbrecht, Germany) and sedimented at $100 \mathrm{~g}$. The pellet was washed twice with PBS before the cells were resuspended in freezing medium. Cells were aliquoted into cryogenic storage vials. The vials were placed in an isolated box in a $-80^{\circ} \mathrm{C}$ freezer to slowly freeze at a rate of approximately $1{ }^{\circ} \mathrm{C} / \mathrm{min}$, then the vials were transferred to liquid nitrogen storage.

\subsubsection{Creation of Stable Mammalian Cell Lines}

For endogenous tagging of vimentin with CRISPR/Cas-9 genome editing, U2OS cells were cotransfected with $2 \mu \mathrm{g}$ of the vimentin-V410-donor plasmid (P2691) and $2 \mu \mathrm{g}$ of the plasmid px330-VIM-gRNA (P2463) [102, 101]. The plasmid px330-VIMgRNA was provided by Dr. Michael Ratz and encoded for the gRNA GCGCAAGATAGATTTGGAAT and the Cas-9 protein.

For the creation of stable landing pad cell lines a HeLa cell line with a CAG promoter and a Bxb1 attP site in the AAVS1 locus was used. The cell line was provided by Dr. Isabelle Jansen [61]. For integration into the Bxb1 attP site, $1 \mu \mathrm{g}$ of the respective integration plasmid (P2432 or P2690) were contransfected with $1 \mu \mathrm{g}$ of the plasmid pCAG-NLS-HA-Bxb1 [54]. The plasmid pCAG-NLS-HA-Bxb1 was a gift from Pawel Pelczar (Addgene \#51271) and encoded for the transient expression of the $\mathrm{Bxb} 1$ recombinase.

After transfection, the cells were cultured for one week. Subsequently, a singlecell sort of fluorescent cells into 96-well plates was performed using flow cytometry (see chapter 2.5.8 for details). The 96-well plates were further cultivated for two to three weeks, then colonies were analyzed by fluorescence microscopy (Lionheart FX automated microscope, BioTek, Winooski, VT, USA) and colonies showing NIR fluorescence combined with the correct localization pattern were isolated and frozen for long-time storage and use in further experiments.

\subsection{Protein Expression and Purification}

\subsubsection{Transformation of Bacterial Cells}

Plasmid DNA was transformed into the appropriate electrocompetent E. coli strain following a method described by Dower et al. [31]. Electrocompetent E. coli cells of various strains were produced from $500 \mathrm{ml} \mathrm{LB}$ culture inoculated with $5 \mathrm{ml}$ over night culture. The culture was grown at $37^{\circ} \mathrm{C}$ until $O D_{600 \mathrm{~nm}}$ reached 0.6 to 1.0 and then cooled on ice. Cells were harvested at $3079 \mathrm{rcf}$ using a Sorvall RC6 Plus centrifuge with a HS-4 rotor (Thermo Fisher Scientific, Waltham, MA, USA). The cells were resuspended in $500 \mathrm{ml}$ sterile water, then centrifuged again and resuspended in $250 \mathrm{ml}$ sterile water. Followed by another centrifugation step and resuspension of the pellet in $10 \mathrm{ml} \mathrm{10 \%} \mathrm{glycerine.} \mathrm{After} \mathrm{a} \mathrm{last} \mathrm{centrifugation} \mathrm{step,} \mathrm{cells} \mathrm{were} \mathrm{resus-}$ pended in $1 \mathrm{ml} 10 \%$ glycerine and stored in aliquots of $50 \mu \mathrm{l}$ at $-80^{\circ} \mathrm{C}$.

For transformation, an aliquot of electrocompetent $E$. coli cells was thawed slowly on ice. Then the cells were resuspended in $50 \mu \mathrm{l}$ sterile water and transferred to an electroporation cuvette (Cell Projects, Herrietsham, UK). An appropriate volume of DNA was added, $5 \mu \mathrm{l}$ for mutagenesis and cloning samples and $1 \mu \mathrm{l}$ for plasmid 
DNA. Electroporation was performed using a pulse controller (BioRad, Hercules, CA, USA) set to $200 \Omega$ and a gene pulser set to $25 \mu \mathrm{F}$ (BioRad, Hercules, CA, USA). After transformation, $1 \mathrm{ml}$ LB medium was added and cells were incubated for $1 \mathrm{~h}$ at $37^{\circ} \mathrm{C}$. Thereafter, the cells were plated on agar plates or added to liquid medium with the appropriate antibiotics. The cells were incubated for 18 to $24 \mathrm{~h}$ at $37^{\circ} \mathrm{C}$. If protein expression was desired for screening experiments or protein purification, $0.02 \%$ arabinose was added to the plates or the liquid medium.

\subsubsection{Expression and Purification}

Depending on the desired amount of protein, E. coli BL21-AI cells (Invitrogen, Carlsbad, CA, USA) transformed with the appropriate $\mathrm{pBad}$ expression plasmid (Thermo Fisher Scientific, Waltham, MA, USA) were grown in either $50 \mathrm{ml}$ liquid medium for small scale purification or 11 liquid medium for large scale purification at $37^{\circ} \mathrm{C}$ for 18-24 h. For small scale purification the liquid medium consisted of LB medium supplemented with $50 \mu \mathrm{g} / \mathrm{ml}$ ampicilin and $0.02 \%$ arabinose for induction of protein expression. For large scale expression $0.02 \%$ arabinose was added not until the $O D_{600 \mathrm{~nm}}$ reached 0.6 to 0.7 . After expression, cells were harvested by centrifugation using either a Heraeus MULTIFUGE $1 \mathrm{~s}$ (Thermo Fisher Scientific, Waltham, MA, USA) for small scale purification or a Sorvall RC6 Plus centrifuge equipped with an SS-34 rotor (Thermo Fisher Scientific, Waltham, MA, USA) for large scale purifications. Cells were resuspended in an appropriate amount of binding buffer before addition of lysozyme (Serva electrophoresis, Heidelberg, Germany) to a final concentration of $1 \mathrm{mg} / \mathrm{ml}$ and incubation on ice for $4 \mathrm{~h}$. Afterwards, cell lysis was performed by repeated freeze and thaw cycles for small scale purification and by sonification using a Branson 450 Digital Sonifier (Branson Ultrasonic, Danbury, CT, USA) for large scale purification. $0.5 \mu$ benzonase (Thermo Fisher Scientific, Waltham, MA, USA) was added to the samples. To remove cell fragments from the supernatant containing the expressed protein the samples were centrifuged for 3 to $6 \mathrm{~h}$ at $4{ }^{\circ} \mathrm{C}$ at $21,000 \mathrm{rcf}$ using a Heraeus Fresco 21 micro centrifuge (Thermo Fisher Scientific, Waltham, MA, USA). The supernatant was removed for further purification by Ni NTA affinity purification while the pellet was discarded.

For small scale purification, the protein was further purified using the His Spin kit (GE Healthcare, Chicago, IL, USA). All following centrifugation steps were carried out at $100 \mathrm{rcf}$ for $1 \mathrm{~min}$ using a Heraeus Pico 17 centrifuge (Thermo Fisher Scientific, Waltham, MA, USA). The His Trap columns were equilibrated by an initial washing step with $600 \mu \mathrm{l}$ binding buffer. Subsequently, $600 \mu \mathrm{l}$ of the supernatant was added to the equilibrated column. Columns were rotated for $30 \mathrm{~min}$ in the dark at room temperature to bind the protein, before centrifugation and removal of the flow through. This step was repeated until all supernatant was applied to the column. Afterwards, the columns were washed twice with $600 \mu \mathrm{l}$ binding buffer and twice with $200 \mu \mathrm{l}$ wash buffer. For elution, $200 \mu \mathrm{l}$ elution buffer was applied and after 3 min incubation the protein was eluted via centrifugation. This step was performed 3 times or until the His Trap column appeared colorless.

For large scale purification, empty $10 \mathrm{ml}$ columns (Mo Bi Tec, Göttingen, Germany) were loaded with an appropriate amount of Ni NTA agarose beads (Qiagen, Hilden, Germany) (binding capacity $50 \mathrm{mg} / \mathrm{ml}$ ). The columns were equilibrated with $5 \mathrm{ml}$ binding buffer. Afterwards, the supernatant was loaded onto the columns. This step was repeated until the flow through appeared colorless. To wash 
the columns, $10 \mathrm{ml}$ binding buffer was applied followed by $10 \mathrm{ml}$ washing buffer. The protein was eluted by applying an appropriate amount of elution buffer until the Ni NTA beads appeared colorless.

After purification, samples were concentrated using Vivaspin ${ }^{\circledR} 500$ centrifugal concentrators (Sartorius, Göttingen, Germany) with a molecular cut-off at $100 \mathrm{kDa}$. The elution buffer was exchanged for standard protein buffer using NAP-5 columns (GE Healthcare, Chicago, IL, USA) following the manufacturers instructions.

\subsection{Protein Characterization}

In this section, different methods are described that were used to characterize the biophysical properties of fluorescent proteins. Those methods include spectral characterizations, chromatography and electrophoresis.

\subsubsection{Measurement of Absorption Spectra}

To measure absorption spectra, a purified protein solution in standard protein buffer at pH 7.5 was used. Samples were measured using a quartz cuvette with $1 \mathrm{~cm}$ path length at a Carry $4000 \mathrm{UV} /$ vis spectrometer (Varian, Palo Alto, CA, USA) in the range between 250 to $650 \mathrm{~nm}$. All absorption spectra depict in this work represent single measurements.

\subsubsection{Measurement of Excitation and Emission Spectra}

Emission and excitation spectra were measured with purified protein solution in standard protein buffer at $\mathrm{pH} 7.5$ using a Carry Eclipse fluorescence spectrometer (Varian, Palo Alto, CA, USA). Excitation spectra were recorded between 500 to $700 \mathrm{~nm}$ with a fixed emission at $710 \mathrm{~nm}$. Emission spectra were recorded between 680 to $850 \mathrm{~nm}$ with a fixed excitation at $670 \mathrm{~nm}$. The emission and excitation spectra that are shown in this work depict single measurements.

\subsubsection{Measurement of Fluorescence Lifetime}

The fluorescence lifetime was measured either by Dr. Alexey Chizhik using the method described by Chizhik et al. [22] or using a Quantaurus-Tau fluorescence lifetime spectrometer (Hamamatsu, Hamamatsu City, Japan) using purified protein in standard protein buffer at $\mathrm{pH}$ 7.5. For measurements performed with the Quantaurus-Tau system, exponential models were fitted to the data using the software provided with the machine.

\subsubsection{Measurement of Fluorescence Quantum Yield}

The fluorescence quantum yield was measured either by Dr. Alexey Chizhik based on the modification of radiative transitions of emitters in a nanocavity as described by Chizhik et al. [22, 23] or using a Quantaurus-QY system (Hamamatsu, Hamamatsu City, Japan). For both methods, purified protein solution in standard protein buffer was used. 


\subsubsection{Measurement of pH Stability}

The $\mathrm{pH}$ stability of fluorescent proteins was measured using the Cytation3 plate reader (BioTek, Winooski, VT, USA) with fluorescence excitation at $650 / 19 \mathrm{~nm}$ and detection at $700 / 19 \mathrm{~nm}$. The protein concentration was $0.1 \mu \mathrm{g} / \mu \mathrm{l}$. The measurements were performed in replicates of $n=3$. The data were analyzed using custom Python software. The fluorescence signal of each measurement were normalized to the corresponding maximal value. The $\mathrm{pk}_{\mathrm{a}}$ values were determined by fitting a monophasic model to the data using custom Python software (see Appendix C).

\subsubsection{Semi-native Gel Electrophoresis}

One approach to determine the oligomeric state of a fluorescent protein is seminative gel electrophoresis. For this, 2 to $4 \mu \mathrm{g}$ of purified protein in $100 \mathrm{mM}$ Tris $\mathrm{HCl}$, $150 \mathrm{mM} \mathrm{NaCl}$ and $10 \%$ sucrose at $\mathrm{pH} 7.5$ was loaded onto a $15 \%$ polyacrylamide gel containing $0.1 \%$ sodium dodecyl sulfate. The gels were casted and run using the Mini-PROTEAN Tetra handcast system (BioRad, Hercules, CA, USA) at a constant current of $20 \mathrm{~mA}$ for approximately $1 \mathrm{~h}$. The electric current was supplied by a $300 \mathrm{~V}$ power source (VWR, Radnor, PA, USA). As standards, monomeric miRFP703 [119] and dimereic iRFP702 [118] were loaded. After electrophoresis, the gel was analyzed using an Amersham Imager 600RGB (GE Healthcare, Chicago, IL, USA) equipped with a $630 \mathrm{~nm}$ excitation source and a Cy5 filter set.

\subsubsection{Size Exclusion Chromatography}

A second approach to determine the oligomeric state of a fluorescnet protein is size exclusion chromatography (SEC). To do this, an Äkta pure chromatography system (GE Healthcare, Chicago, IL, USA) equipped with a Superdex ${ }^{\mathrm{TM}} 200$ Increase 10/300 column (GE Healthcare, Chicago, IL, USA) was used. Before applying the purified protein solution to the chromatography system, the solution was filtered using VIVASPIN 500 columns with $0.2 \mu \mathrm{m}$ PES membranes (Satorius, Göttingen, Germany). Subsequently, $250 \mu \mathrm{l}$ protein solution in standard protein puffer at a concentration of $10 \mu \mathrm{M}$ was applied to the Superdex ${ }^{\mathrm{TM}}$ column and eluted at a flow rate of $0.75 \mathrm{ml} / \mathrm{min}$ using standard protein buffer. The protein was detected using a UV monitor U9-L (GE Healthcare, Chicago, IL USA) operating at a fixed wavelength of $280 \mathrm{~nm}$. As standards, monomeric miRFP703 [119] and dimereic iRFP702 [118] were measured. All measurements were performed at $6^{\circ} \mathrm{C}$.

\subsubsection{Flow Cytometry}

In order to measure the brightness of proteins in cells or for fluorescence activated cell sorting, flow cytometry was performed. The measurements were done using a modified flow cytometry system (BD influx ${ }^{\mathrm{TM}}$ cell sorter, BD Biosciences, Franklin Lakes, NJ, USA) with a $671 \mathrm{~nm}$ laser (SDL-671-300T, Shanghai Dream Laser, Shanghai, China) for NIR excitation and a $488 \mathrm{~nm}$ laser (Genesis CX488; Coherent, Santa Clara, CA, USA) for green excitation. The NIR fluorescence was detected in two windows between 690 to $720 \mathrm{~nm}$ and 720 to $766 \mathrm{~nm}$ and the green fluorescence was detected between 495 to $525 \mathrm{~nm}$.

The cells were cultured in 6-well plates until appropriate density. Approximately $2 \mathrm{~h}$ before flow cytometry measurements, biliverdin was added to the cells. Immediately before the measurements, the DMEM culture medium was removed and the 
cells were washed twice in PBS. Subsequently, trypsin was added to the cells for 10 to $15 \mathrm{~min}$ to detach them from the bottom of the wells. Detachment was stopped by adding appropriate amounts of DMEM. Then, cells were transferred into $15 \mathrm{ml}$ tubes and pelleted for $3 \mathrm{~min}$ at $100 \mathrm{~g}$. Pellets were washed twice in PBS and subsequently resuspended in PBS. Before flow cytometry measurements, the cells were filtered.

\subsection{Fluorescence Lifetime Screening}

In order to increase the fluorescence lifetime and the fluorescence quantum yield of an NIR FP template, a directed evolution approach was applied combined with an automated microscope for selection of improved protein variants with increased fluorescence lifetime. The automated fluorescence microscope is described in chapter 2.6.1 and the directed evolution workflow is described in chapter 2.6.2.

\subsubsection{Automated Fluorescence Lifetime Screening Microscope}

A customized automated screening microscope was used for automated measurements of diversified protein pools expressed in E. coli colonies growing on agar plates. An optical plan of the automated screening microscope is shown in figure 2.1. A large part of the customization of the automated microscope was done prior to this work by Dr. Martin Andresen and Dr. Timo Konen [69]. Part of this work was the improvement of the optical system, the electronics and the software in order to enable automated fluorescence lifetime measurements.

The microscope body was taken from a commercial fluorescence microscope (DM5500 B, Leica Microsystems, Wetzlar, Germany). The microscope was equipped with a pulsed $670 \mathrm{~nm}$ excitation laser (LDH-P-C-670, PicoQuant, Berlin, Germany, maximal pulse rate: $80 \mathrm{MHz}$, output pulse duration $20 \mathrm{ps)}$ operated with a laser driver (PDL 800-B, PicoQuant, Berlin, Germany) at $20 \mathrm{MHz}$. The laser beam was coupled into the microscope via a dichroic mirror (HC BS 685, Semrock, New York, USA) and focused onto the sample using a $20 \mathrm{x}$ air objective lens (NPLANL 20x/0.40, Leica Microsystems, Wetzlar, Germany). The emitted fluorescence light was collected via the same objective lens, filtered by an optical filter (720/60 ET Bandpass, Chroma, Bellows Falls, USA) and focused on an Avalanche photodiode (APD) (MPD-020-CTB, PicoQuant, Berlin, Germany) and through a pinhole on a photomultiplier tube (PMT) (H10723, Hamamatsu, Hamamatsu, Japan). The APD was connected to time correlated single photon counting (TCSPC) electronics (SPC-830, Becker \& Hickel, Berlin, Germany) for the temporally resolved detection of single photons. The microscope and the electronic stage (SCAN 100x100, Märzhäuser Wetzlar, Wetzlar, Germany) were controlled by a microscope controller box (CTR6500, Leica Microsystems, Wetzlar, Germany). The automation of the system was implemented using custom LabVIEW code (National Instruments, Austin, TX, USA). The TCSPC module was integrated using a MATLAB ${ }^{\circledR}$ library provided by Dr. Marcel Leutenegger. Fitting and analysis of fluorescence lifetime data was performed with MATLAB $^{\circledR}$ code following a method described by Enderlein et al. [37].

\subsubsection{Directed Evolution}

In order to successively improve the parameters fluorescence lifetime and fluorescence brightness of a fluorescent protein, the approach of directed evolution was applied in this work. The schematic workflow is depicted in figure 2.2. 


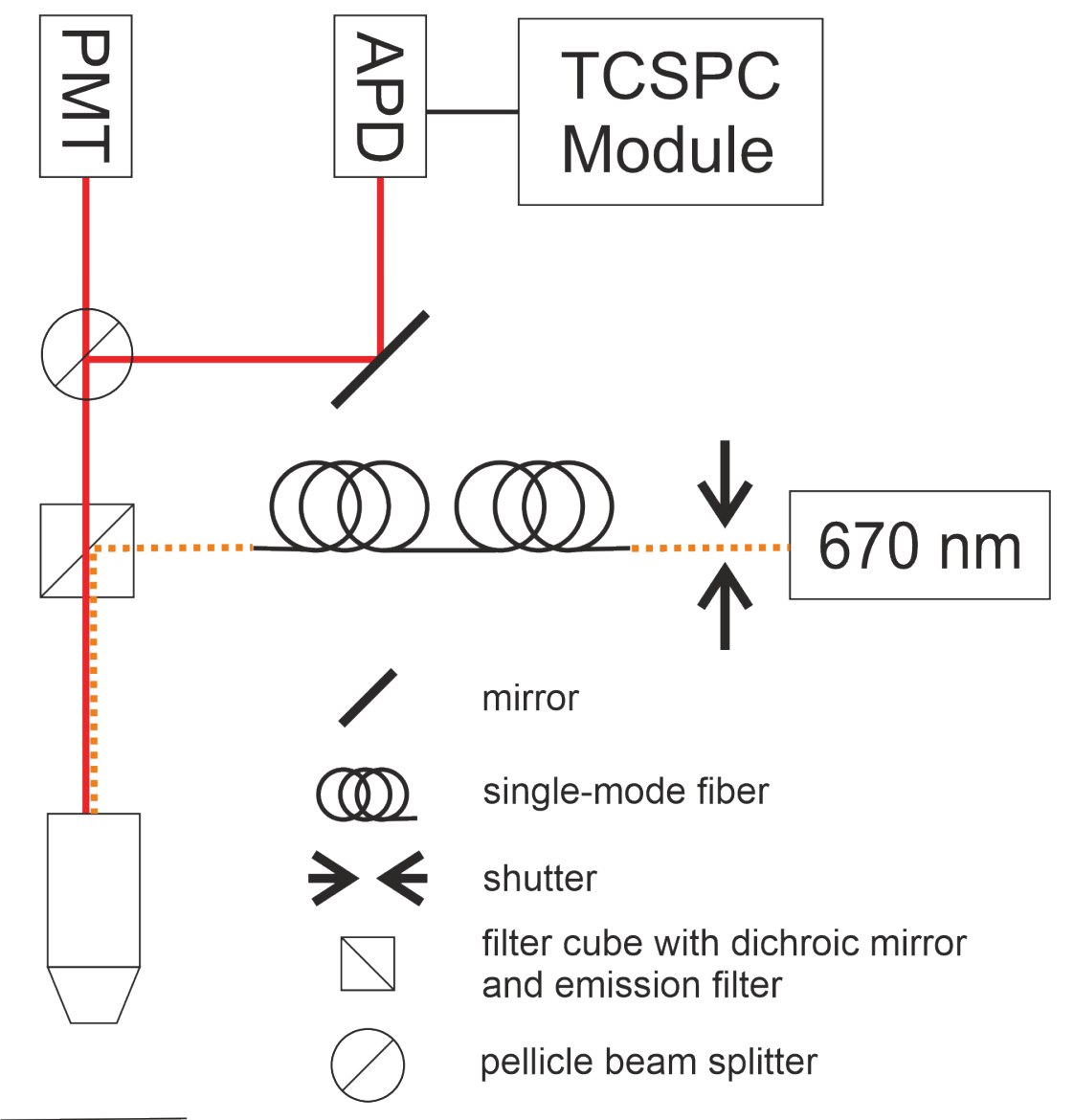

FIGURE 2.1: Schematic drawing of fluorescence lifetime screening microscope. The plan depicts laser beam path (dashed line), detection path (solid line) and position of optical elements. PMT, photon multiplier tube; TCSPC Module, time correlated single photon counting module; APD, Avalanche photodiode.

In the fist step, the starting template or an intermediate protein variant was diversified using PCR based techniques, e.g. site directed (see chapter 2.1.3), multiple site (see chapter 2.1.4) or error prone mutagenesis (see chapter 2.1.5). Subsequently, the diversified protein pool was transformed into electrocompetent E. coli BL21AI cells and the cells were plated on agar plates with appropriate antibiotics and $0.02 \%$ arabinose. Since E. coli does not have a dedicated BV metabolism heme oxygenase-1 (HO-1) from the cyanobacterium Synechocystis was coexpressed together with the respective protein variant. For this, the coding sequence of the fluorescent protein smURFP from the plasmid pBAD-smURFP-RBS-HO-1 (Addgene \#80341 [109]) was substituted by the desired protein variant. After incubation for 18 to $24 \mathrm{~h}$ at $37^{\circ} \mathrm{C}$, the brightness and fluorescence lifetime of bacterial colonies growing on the agar plates was measured using the automated fluorescence microscope described in chapter 2.6.1. Colonies expressing protein variants with increased brightness and/or fluorescence lifetime were transferred in $5 \mathrm{ml}$ LB medium with appropriate antibiotics and incubated for 18 to $24 \mathrm{~h}$ at $37^{\circ} \mathrm{C}$. Thereafter, the plasmid DNA was isolated from the $5 \mathrm{ml}$ cultures using the QIAprep Spin Miniprep Kit (Qiagen, Hilden, Germany). The isolated DNA of the improved protein variants was pooled and another round of PCR based diversification and selection was performed. 


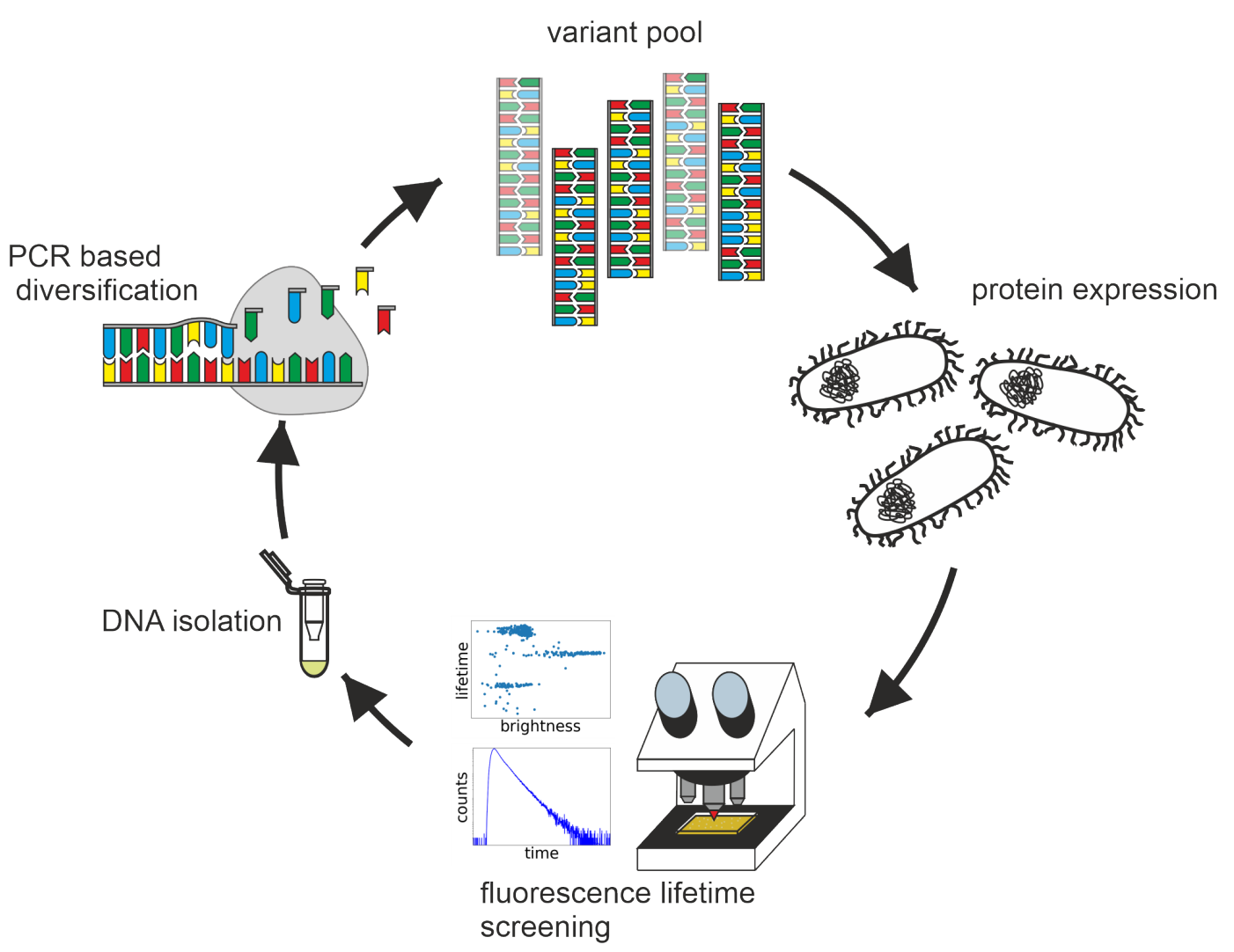

FIGURE 2.2: Workflow of directed evolution approach.

\subsection{Microscopy}

Approximately $48 \mathrm{~h}$ before imaging, mammalian cells were seeded in 6-well plates (Sarstedt, Nümbrecht, Germany) on $18 \mathrm{~mm}$ glass coverslips No. 1.5H (Paul Marienfeld, Lauda-Königshofen, Germany). $24 \mathrm{~h}$ hours before the imaging experiment, the cells were transfected with 1 to $3 \mu \mathrm{g}$ of the desired plasmid using TurboFect Kit (Thermo Fisher Scientific, Waltham, MA, USA) following the manufacturers instructions. Around 2 to $3 \mathrm{~h}$ before the imaging experiment, up to $25 \mu \mathrm{M}$ biliverdin was added to the cells. Immediately before fluorescence microscopy measurements, the coverslips with the attached cells were washed in HDMEM (HEPES buffered DMEM) and sealed on a glass slide. The sealed cells were imaged at room temperature on the same day.

All fluorescence microscopy measurements were performed on a commercial QUAD scanning fluorescence microscope (Abberior Instruments $\mathrm{GmbH}$, Göttingen, Germany) utilizing an inverted microscope body (IX83, Olympus, Tokyo, Japan). A Schematic drawing of the microscope is shown in figure 2.3. The microscope was equipped with a titanium-sapphire laser (Mai Tai - Spectra-Physics, Santa Clara, CA, USA, pulse rate: $80 \mathrm{MHz}$, output pulse duration $150 \mathrm{fs}$ ) that was used to generate the excitation and STED beam. The laser beam (mode locked between 790 to $840 \mathrm{~nm}$ ) was split into excitation and STED beam using a polarizing beam splitter. One polarization was then fed into a photonic crystal fibre (Femto WHITE 800, NKT Photonics A/S, Birkerød, DK) to generate a white light spectrum. The excitation spectrum was selected with a tunable band pass filter (VersaChrome HC 708/13, Semrock, New York, USA). The other polarization was used to produce the STED beam. For this, 
the laser pulse from the titanium-sapphire laser was stretched utilizing glass blocks as dispersive media. The exact length of the laser pulse was adjusted choosing a single mode fibre of appropriate length. The timing between the excitation laser pulse and the STED pulse was adjusted using an optical delay line. The spectra of the excitation and the STED beams are shown in figur D.1. Both beams were focused into the sample and fluorescence light was collected using an oil immersion objective lens (UPlanSApo $100 \mathrm{x} / 1.4$ Oil, Olympus, Tokyo, Japan) together with the supplied immersion oil. NIR fluorescence light between 680 to $750 \mathrm{~nm}$ was focused through a pinhole on a silicon avalanche photodiode (Photon Counting Module SPCM-AQRH13-FC, Excelitas, Waltham, Massachusetts, USA) for confocal and STED imaging and on a prototype superconducting nanowire single photon detector (SNSPD) (SingleQuantum, Delft, Netherlands) connected with a TCSPC system (SPC-150, Becker \& Hickel, Berlin, Germany) for fluorescence lifetime imaging. The microscope was controlled and images were acquired using supplied software (Imspector, Abberior Instruments $\mathrm{GmbH}$, Göttingen, Germany). The exact imaging parameters are presented in table D.1.

\subsubsection{Image Processing and Analysis}

The raw imaging data were exported as msr-files. Processing and analysis was performed with the open source software FIJI [116] or custom Python code. Line profiles were taken with FIJI using a pixel averaging of 3 . The profiles were then fitted in Python using a Lorentzian function (equation (2.1)).

$$
y=\frac{A}{\pi} * \frac{w}{\left(x-x_{0}\right)^{2}+w^{2}}+o f f s e t
$$

The Python code together with a detailed description of the method used for analysis of bleaching, reexcitation and STED efficiency, $\mathrm{pH}$ spectra and decomposition of fluorescence lifetime images can be found in appendix $C$. 


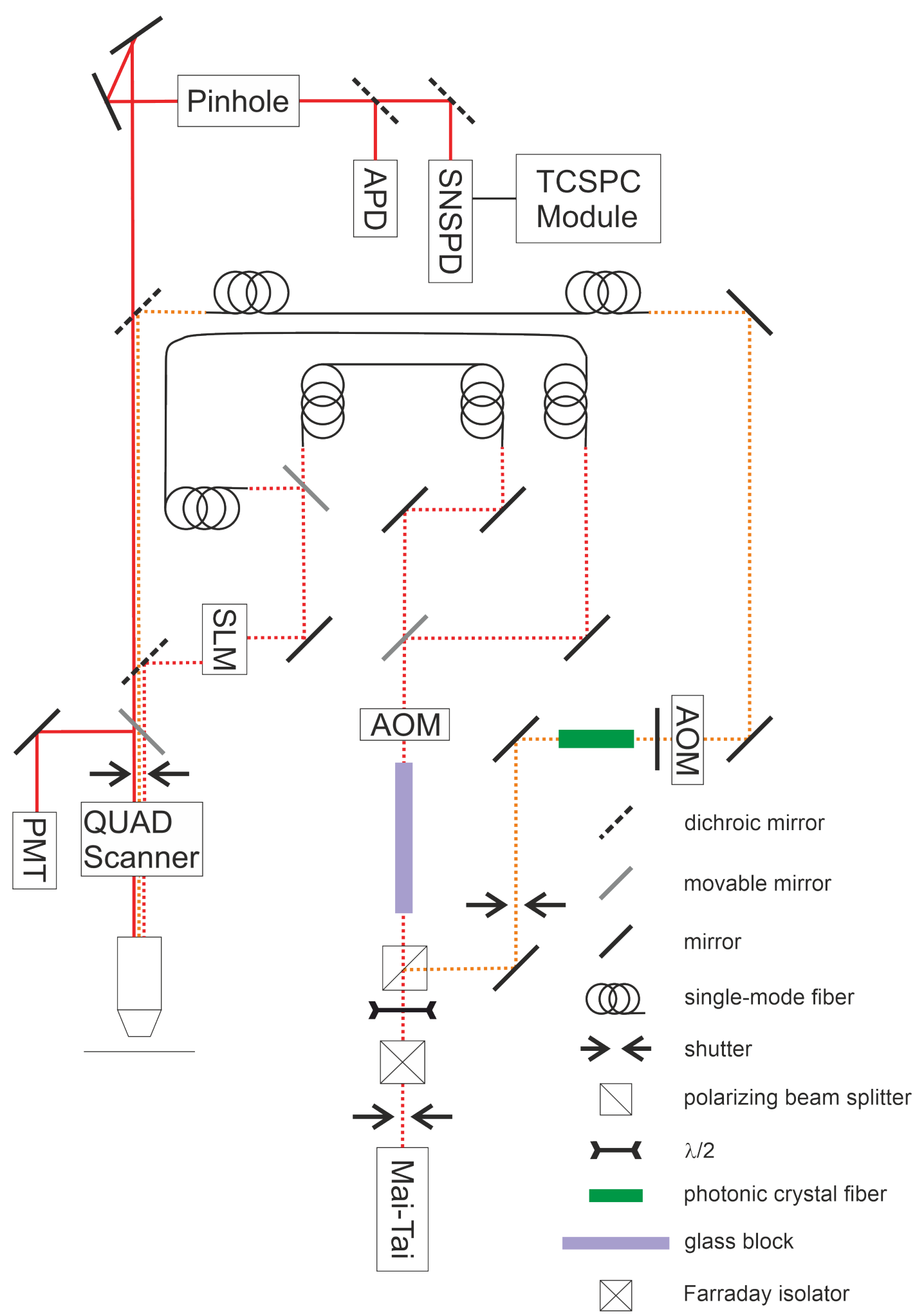

FIGURE 2.3: Schematic drawing of NIR STED microscope. The drawing depicts laser beam paths (STED beam: red dashed lines; excitation beam: orange dashed line), detection path (solid lines) and position of optical elements. SLM, spatial light modulator; AOM, acousto optical modulator; APD, Avalanche photodiode; PMT, photon multiplier tube; TCSPC, time correlated single photon counting; SNSPD, superconducting nanowire single photon detector. 



\section{Chapter 3}

\section{Results}

In this work, the NIR fluorescent protein miRFP703 [119] was improved in regards to an application in fluorescence microscopy. This was done via directed evolution over consecutive rounds of PCR-based diversification, automated screening and selection of variants with increased fluorescence lifetime and fluorescence intensity. The results of this efforts are reported in this chapter. First, the implementation and validation of the automated screening system is described in section 3.1. Afterwards, the results of the screening are reported in section 3.2. The most beneficial protein variant is characterized biophysically in section 3.3. In section 3.4, different applications of the variant in live-cell fluorescence microscopy are demonstrated.

\subsection{Implementation of Fluorescence Lifetime Screening}

The starting template miRFP703 was improved using the approach of directed evolution consisting of consecutive rounds of PCR-based diversification and automated selection of improved protein variants (see section 2.6.2). In each round, the pool of template DNA was diversified using site directed, multiple site directed or random site mutagenesis (see section 2.1). The diversification step resulted into a pool of different protein variants. This pool was transformed into E. coli cells and plated on agar plates. Next, improved protein variants had to be isolated based on performance measures that were suitable to predict the performance of the protein in fluorescence live-cell microscopy. For this work, the parameters of fluorescence lifetime and fluorescence intensity in $E$. coli cells were used as performance measures. The parameter of fluorescence lifetime was chosen because it is proportional to the fluorescence quantum yield (compare with equation (1.4)). A high fluorescence quantum yield enables high contrast and low excitation light doses in live-cell imaging. Additionally, increasing the fluorescence lifetime also increases the achievable resolution in STED microscopy (see equation (1.7)). The fluorescence intensity in E. coli cells reflected a mixture of protein folding, maturation and stability and BV affinity of the protein variants. Besides this, the measured fluorescence intensity was also affected by non-protein related factors such as colony size and focus position. Therefore, this parameter was expected to show considerable variance. However, the parameter was included into the screening, to have a measure for the effective brightness of the protein variants at $37^{\circ} \mathrm{C}$. In conclusion, the working hypothesis was that the combined screening for protein variants with increased fluorescence lifetime and fluorescence intensity in E. coli cells would yield proteins with high fluorescence quantum yields, high BV affinity and good folding, maturation and stability properties at $37^{\circ} \mathrm{C}$ that perform well in live-cell fluorescence microscopy.

The theoretical number of different protein variants is much higher than one can ever hope to measure. The protein miRFP703 has 315 amino acids. Therefore, there 
are $315^{20}$ different possible protein sequences. The task of improving a fluorescent protein can be viewed as finding maxima in this high dimensional fitness landscape. In practice, only a fraction of possible protein variants can be explored. To sample as many variants as possible, a commercial fluorescence microscope was customized to automatically measure the fluorescence lifetime and the fluorescence intensity of E. coli colonies expressing a protein variant. For this, the microscope was upgraded with a movable electronic stage, a pulsed NIR laser system, an APD detector and a TCSPC timing electronics as described in section 2.6.1. Custom LabVIEW and MATLAB software was used to control the hardware and analyze the recorded data. Prior to this work, Dr. Martin Andresen implemented the electronics to control the microscope via LabVIEW software and Dr. Timo Konen [69] implemented methods for fast sampling and autofocus. With this screening microscope in place several thousand colonies could be measured automatically per day.

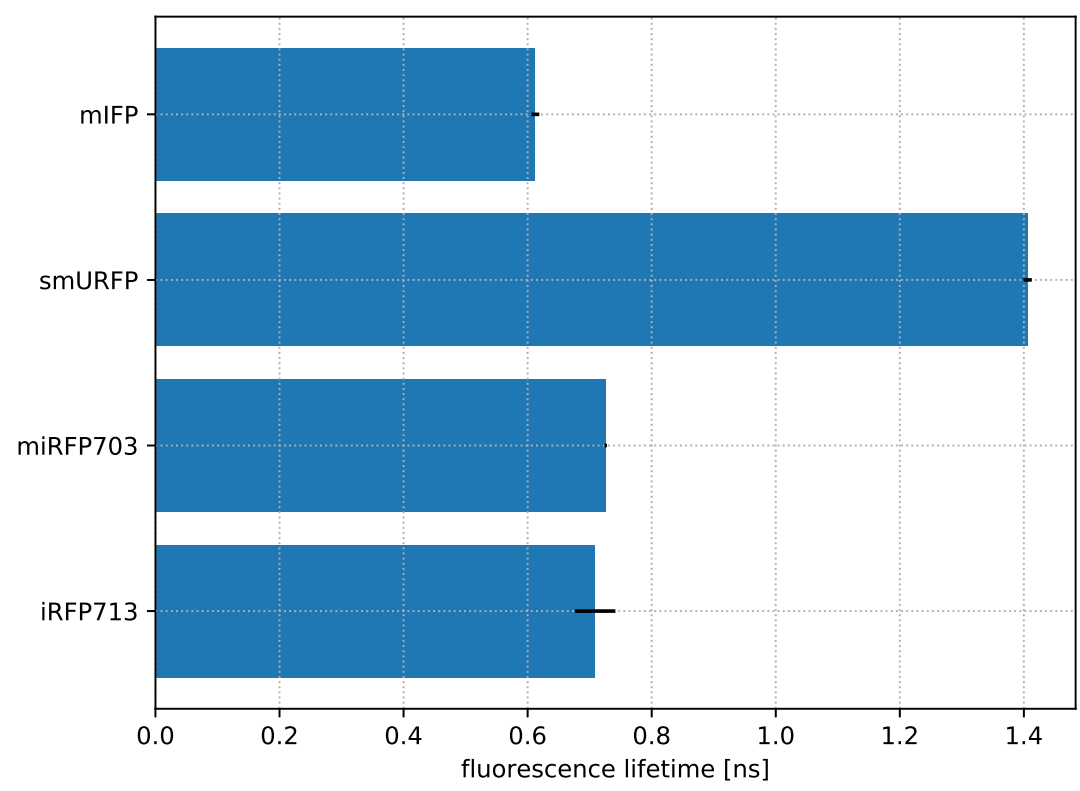

FIGURE 3.1: Fluorescence lifetimes of different NIR FPs measured with the automated screening microscope. The standard deviations are indicated by black bars.

To evaluate the precision of the fluorescence lifetime measurement of the automated screening microscope, four different engineered NIR fluorescent proteins expressed in E. coli cells growing on agar plates were measured with five replicates. Figure 3.1 shows the mean fluorescence lifetimes of the proteins. The black bars indicate the standard deviation. For all proteins, the standard deviation was below $5 \%$ indicating a reliable performance of the fluorescence lifetime measurement and the fitting procedure.

To test the performance of the automated microscope under screening conditions, three different engineered fluorescent proteins were transformed into E. coli cells. The cells were mixed and plated on an agar plate. More than 500 colonies growing on the agar plate were measured automatically with the screening microscope. The data points were clustered with Python and scikit-learn [19] using a 
gaussian mixture model with three centres. Figure 3.2 shows the result. Three clusters are clearly separated from each other representing the three different fluorescent proteins. The centres of the clusters are at $0.88 \mathrm{~ns}|0.23,0.54 \mathrm{~ns}| 0.11$ and $1.10 \mathrm{~ns} \mid 0.13$, respectively. The fluorescence intensities of the clusters strongly overlapped, while the fluorescence lifetimes were well separated.

In summary, the automated screening microscope proved capable of automatically measure the fluorescence lifetime and the fluorescence intensity of several thousand E. coli colonies per day expressing a pool of protein variants. The measurements proved reliable and it was possible to reconstruct clusters from a pool of protein variants.

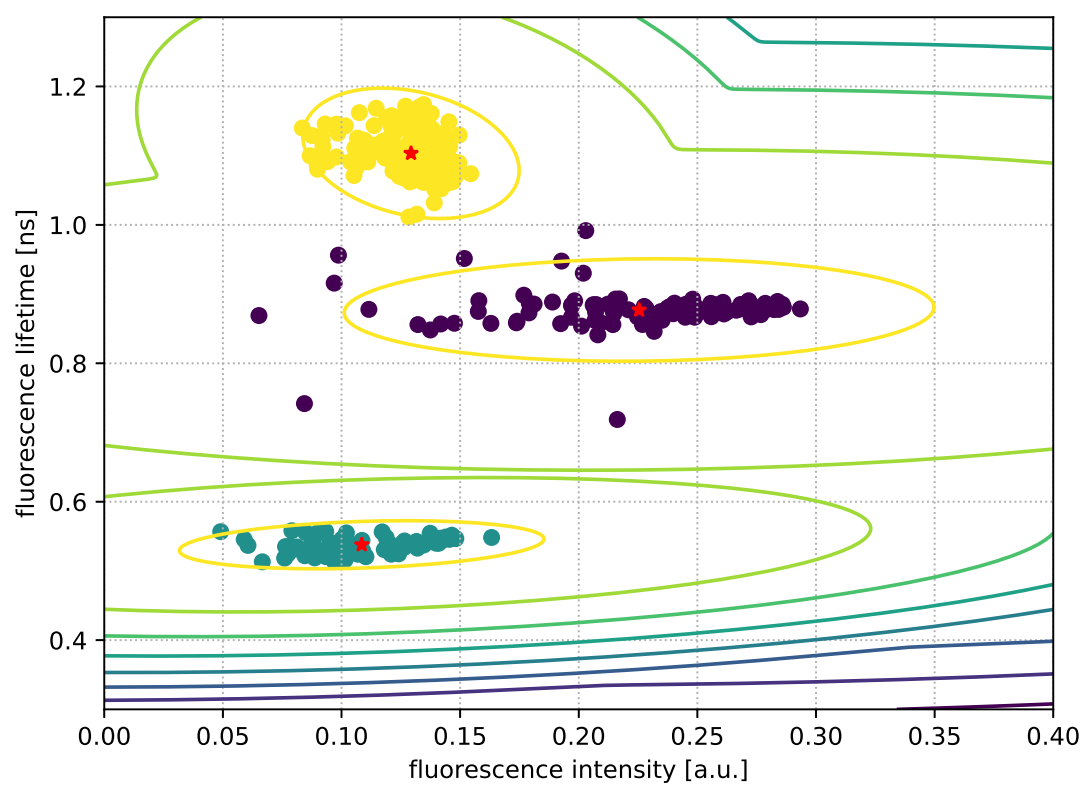

FIGURE 3.2: Test screen of protein pool consisting of 3 different NIR FPs. The data were clustered using a gaussian mixture model with three centres. The data points were colored in accordance to their obtained cluster affiliation.

\subsection{Directed Evolution of miRFP703 to Increase Lifetime and Quantum Yield}

In this work, the fluorescence lifetime and quantum yield of the NIR fluorescent protein miRFP703 was gradually increased over consecutive rounds of diversification and selection. In the first rounds of diversification and selection, amino acids in close proximity to the BV chromophore and positions known from the literature were targeted specifically using site directed and multiple site directed mutagenesis. Positions within a radius of $5 \AA$ around the BV chromophore are positions 19, 23, 25, 168, 170, 180, 192, 197, 200, 201, 202, 203, 205, 206, 210, 216, 248, 249, 250, 251, 253, $254,257,258,261,266,268,280,282$ and 284 . Figure 3.3 shows the location of those amino acids relative to the chromophore. Positions from the literature that were selected for directed mutagenesis were positions 264, 274, 284, 290 and 311 described by Kamper et al. [65] and positions 16, 17, 22, 43, 47, 106, 127, 155, 179, 184, 207, 211, 230, 260, 268, 281, 292, 300, 301 and 305 described by Shcherbakova et al. [119]. 
In general, for directed mutagenesis primers were used with random nucleotides at the positions to mutate.

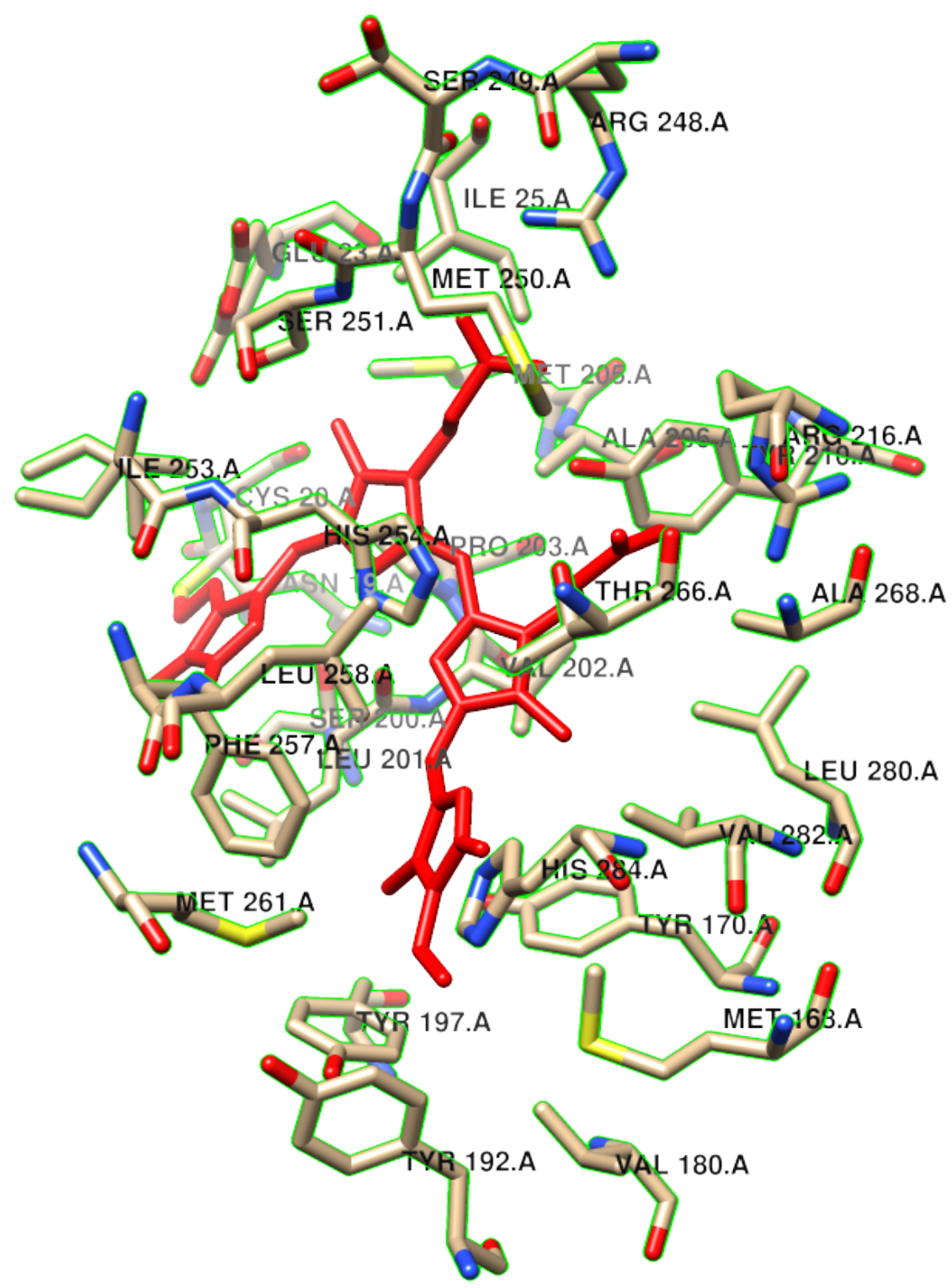

FIGURE 3.3: Amino acid site chains within a radius of $5 \AA$ around the BV chromophore in the crystal structure of miRFP703. The respective site chains are shown in stick representation and are colored by heteroatoms (sulfur: yellow, oxygen: red, nitrogen: blue). The chromophore is shown in stick representation and colored in red. The figure is based on PDB structure 5VIK [119].

Additionally, the position of the BV binding cysteine residue was altered. In the PAS domain, the positions 18, 19, 20 and 21 and in the GAF domain positions 252, 251, 252, 253, 254 and 255 were explored for alternative BV binding. It turned out that all alternative BV binding positions strongly affected the fluorescence properties of the protein. However, the resulting parameters of the variants were not beneficial. Therefore, no protein variant with alternative BV binding sites was used for further experiments. 
From the first rounds of site directed diversification the single mutant I253S stood out. It showed a strongly increased fluorescence lifetime (918 ps) and quantum yield $(18 \%)$. However, this did not translate into a likewise increased fluorescence intensity in E. coli cells. It was hypothesised that by increasing the fluorescence lifetime, and with this the fluorescence quantum yield, either protein expression, folding, maturation or BV affinity was decreased. To rescue the brightness in E. coli cells, several rounds of random site mutagenesis were performed. Thereafter, the final variant showed a three times higher fluorescence brightness in the E. coli expression system as compared to the template miRFP703, accompanied by a further increase of fluorescence lifetime. The final protein variant was termed V410.

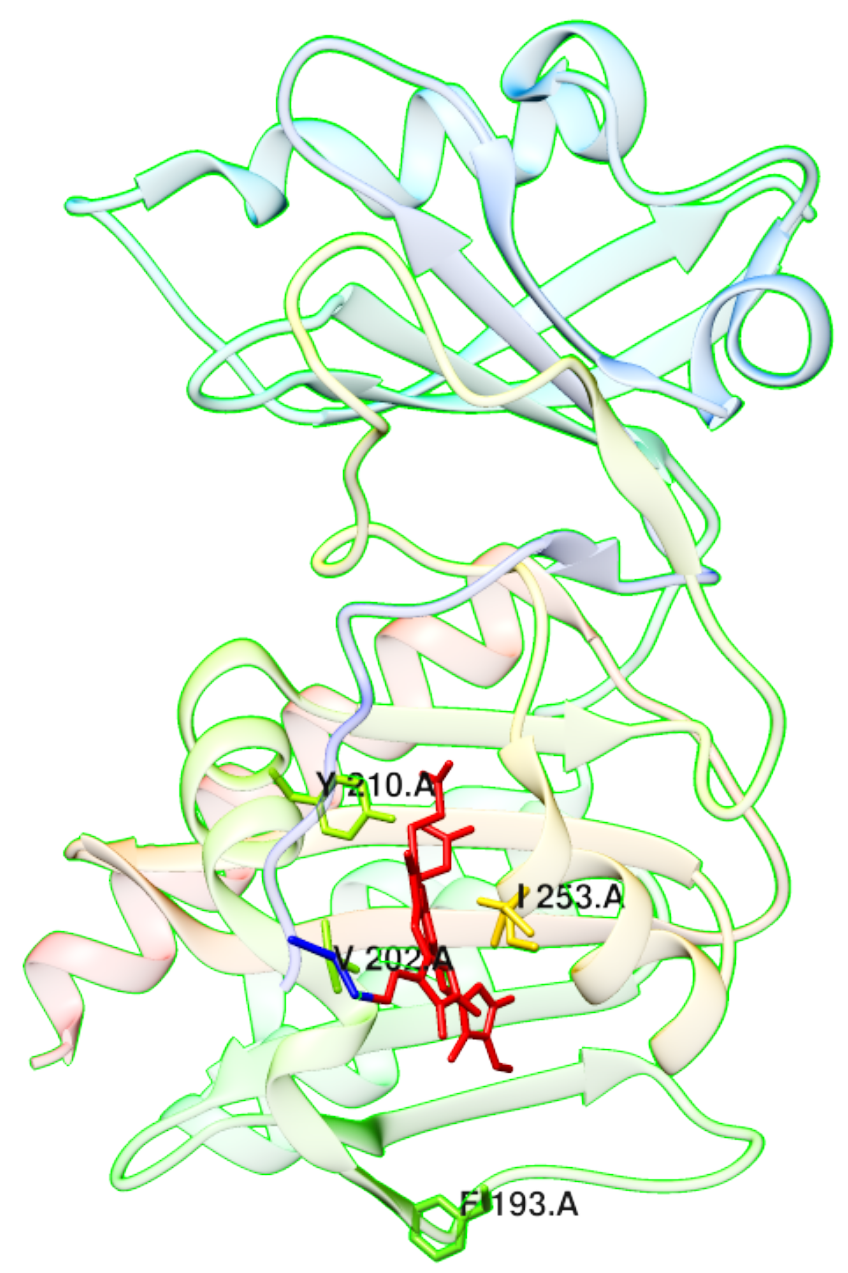

FIGURE 3.4: Position of mutations indicted on the crystal structure of miRFP703. The protein scaffold is shown in cartoon representation and rainbow color scheme ( $\mathrm{N}$-terminus, blue to $\mathrm{C}$-terminus, red). The chromophore is colored in red. Mutations introduced to the protein to form protein variant $\mathrm{V} 410$ are shown in stick representation and are labeled. The figure is based on PDB structure 5VIK [119].

The final protein variant V410 had 4 mutations, namely F193L, V202C, Y210H and I253S. In figure 3.4 the positions of the mutations are marked in the crystal structure of miRFP703. The fluorescence lifetimes and the absorption, excitation and emission spectra of the template miRFP703, the single mutants F193L, V202C, $\mathrm{Y} 210 \mathrm{H}$ and I253S and the final protein variant are shown in figure 3.5, figure 3.6a, 
figure 3.6b and figure 3.6c, respectively. Table D.2 summarizes the results. Mutation I253S is located in the GAF domain in close proximity to the BV chromophore. This mutation alone increased the fluorescence lifetime of miRFP703 from around $700 \mathrm{ps}$ to over $900 \mathrm{ps}$. However, only the synergistic effects of the all four mutations combined yielded a fluorescence lifetime of over $1.1 \mathrm{~ns}$ for the variant V410. The overall shape of the absorption spectra did not change for the single mutants and the final protein variant compared to the template miRFP703 (see figure 3.6a). All spectra exhibit a peak around $280 \mathrm{~nm}$ from the absorption of aromatic amino acids. The so called Soret peak around $380 \mathrm{~nm}$ and the peak around $670 \mathrm{~nm}$ are caused by absorption of the BV chromophore. However, the absorption peak around $670 \mathrm{~nm}$ is broadened for the variants I253S and V410 including a blue shift of $15 \mathrm{~nm}$ as compared to the template miRFP703. The broadening and the blue shift for variants I253S and V410 are also visible in the excitation and emission spectra (see figure $3.6 \mathrm{~b}$ and figure 3.6c). In the case of emission spectra, variants I253S and V410 show a pronounced blue shifted shoulder around $670 \mathrm{~nm}$. Altering the protein concentration had a strong effect on the size of the shoulder. It was more pronounced for low protein concentrations and vanished for higher protein concentrations (compare figure $3.6 \mathrm{c}$ and figure $3.7 \mathrm{~b}$ ).

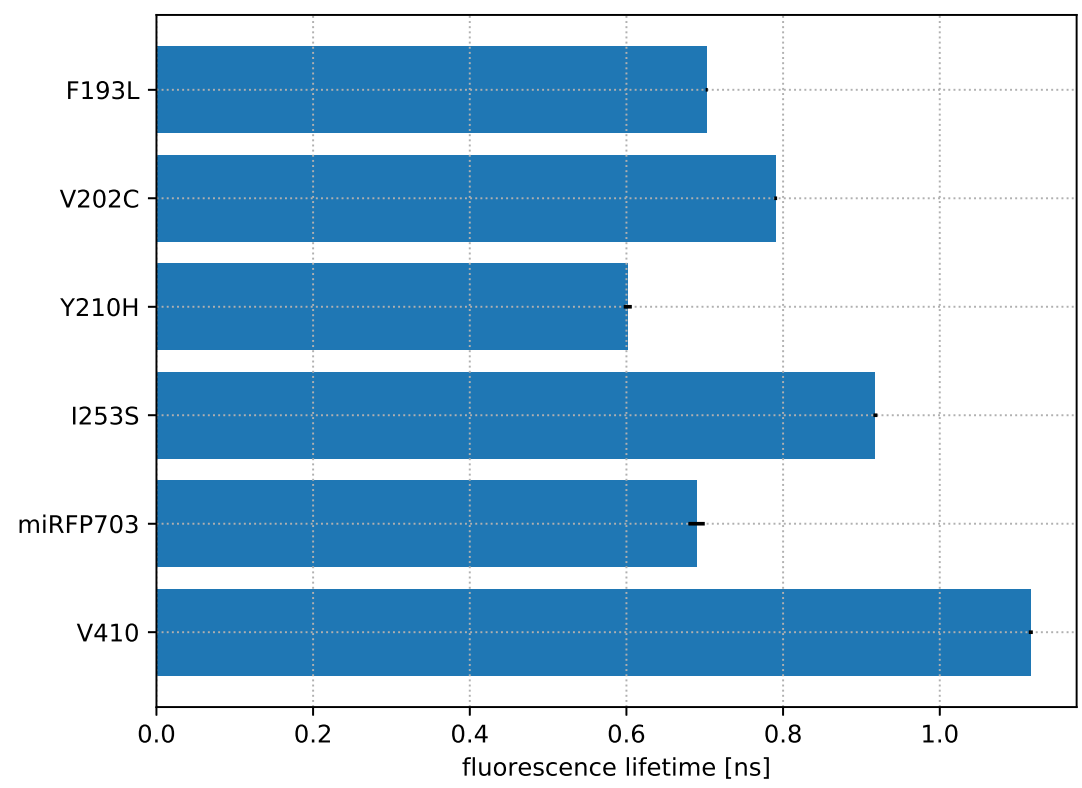

FIGURE 3.5: Comparison between fluorescent lifetimes of single mutant protein variants, final protein variant V410 and template miRFP703. The standard deviations are indicated by black bars. 


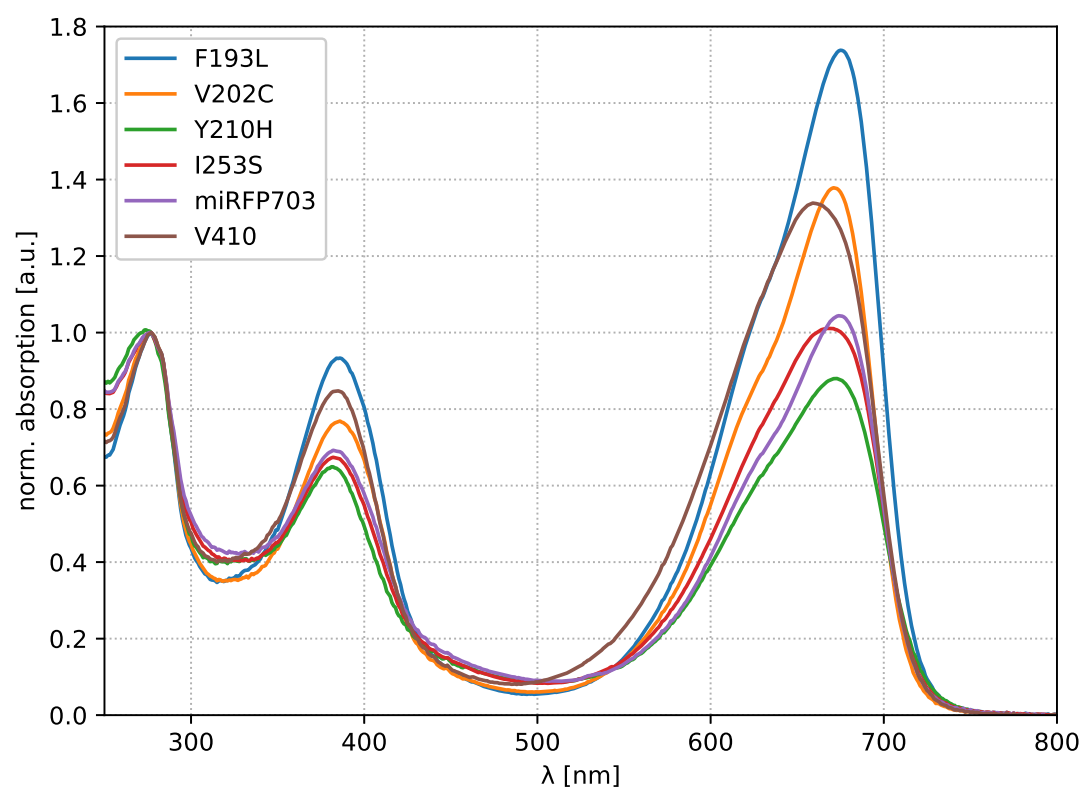

(A) Absorption spectra

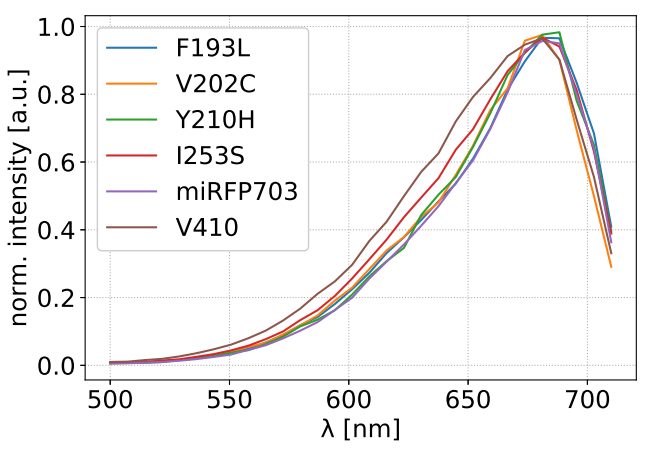

(B) Excitation spectra

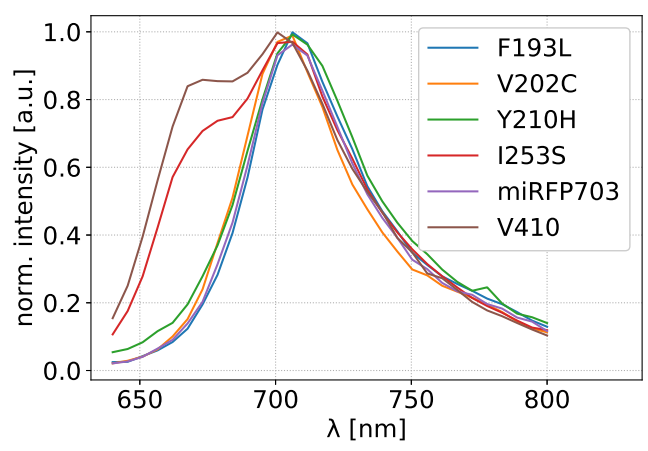

(C) Emission spectra

FIGURE 3.6: Spectral comparison between single mutant protein variants, final protein variant V410 and the template miRFP703. (A) Absorption, (B) excitation and (C) emission spectra of V410, single mutants and miRFP703. For all measurements purified protein in standard protein buffer at $\mathrm{pH} 7.5$ was used.

\begin{tabular}{l|c|l|c} 
Name & $\begin{array}{l}\text { Fluorescence } \\
\text { Lifetime [ps] }\end{array}$ & $\begin{array}{l}\text { Excitation/ } \\
\text { Emission [nm] }\end{array}$ & $\begin{array}{l}\text { Quantum } \\
\text { Yield [\%] }\end{array}$ \\
\hline F193L & $702 \pm 2$ & $681 / 706$ & N/A \\
V202C & $791 \pm 2$ & $681 / 706$ & N/A \\
Y210H & $602 \pm 6$ & $688 / 706$ & N/A \\
I253S & $918 \pm 3$ & $682 / 706(+678)$ & 18 \\
miRFP703 & $690 \pm 13$ & $681 / 706$ & 12 \\
V410 & $1120 \pm 3$ & $681 / 700(+674)$ & 21
\end{tabular}

TABLE 3.1: Comparison of miRFP703 variants 


\subsection{Biophysical Characterization of V410}

The final protein variant V410 was characterized further using standard biophysical techniques. The spectral properties were investigated by fluorescence lifetime measurements, quantum yield measurements and spectroscopic measurements. To determine the $\mathrm{pH}$ stability, the fluorescence intensity was measured in buffers with $\mathrm{pH}$ values ranging from 3 to 9 . The oligomeric state of the protein was investigated using size exclusion chromatography and semi-native gel electrophoresis. For all measurements purified protein solution was used. In the following, the results of the different characterizations are described.

\section{Optical Characterization of V410}

The optical properties of a fluorescent protein are the most important measures for its performance in fluorescence microscopy. In this regard, the protein variant V410 was characterized optically by measuring the absorption, excitation and emission spectra, the fluorescence lifetime and the fluorescence quantum yield. For comparison, the template miRFP703 was characterized alongside V410.

The absorption spectra of V410 and miRFP703 are shown in figure 3.7a. Both exhibit an absorption peak at $280 \mathrm{~nm}$ due to the absorption of aromatic amino acids, e.g., phenylalanine, tryptophan, tyrosine and histidine. Typical for porphyrin compounds is the Soret peak at $380 \mathrm{~nm}$ due to the $\pi-\pi^{*}$ transition of the BV chromophore [126]. The main absorption peak of the BV chromophore bound to the protein had a maximum at $658 \mathrm{~nm}$ and $673 \mathrm{~nm}$ for V410 and miRFP703, respectively. Additionally to the blue shift of $15 \mathrm{~nm}$, the peak was broadened for V410 as compared to miRFP703. From the absorption spectra normalized to the absorption of the aromatic amino acids at $280 \mathrm{~nm}$ the extinction coefficient from V410 was calculated relatively to miRFP703 $\left(\epsilon_{670 \mathrm{~nm}}=90900 \mathrm{M}^{-1} \mathrm{~cm}^{-1}\right.$ [119] $)$ to be approximately $114700 \mathrm{M}^{-1} \mathrm{~cm}^{-1}$.

Figure $3.7 \mathrm{~b}$ shows the excitation and emission spectra of V410 and miRFP703. The excitation maxima were at $677 \mathrm{~nm}$ and $683 \mathrm{~nm}$, while the emission maxima were at $704 \mathrm{~nm}$ and $707 \mathrm{~nm}$ for V410 and miRFP703, respectively. The excitation and emission peaks of V410 were broadened as compared to miRFP703. In comparison to the emission spectrum of V410 shown in figure 3.6c the spectrum in figure $3.7 \mathrm{~b}$ was recorded at a higher protein concentration and did not show the pronounced shoulder at $670 \mathrm{~nm}$.

The fluorescence decay histograms of V410 and miRFP703 were recorded using the Quantaurus-Tau fluorescence lifetime spectrometer and are shown in figure 3.7c. An exponential decay model was fitted to the decay histograms and characteristic fluorescence lifetimes of $1120 \mathrm{ps} \pm 3$ ps for V410 and $690 \mathrm{ps} \pm 13$ ps for miRFP703 were obtained. The fluorescence quantum yields were measured using the QuantaurusQY system and a nanocavity-based system by Dr. Alexey Chizhik [23]. The Quantaurus-QY system yielded quantum yields of 7\% for V410 and 5\% for miRFP703, while Dr. Alexey Chizhik measured values of $21 \%$ for V410 and $12 \%$ for miRFP703. The fluorescence quantum yield values obtained with the Quantaurus-QY system are lower than the values obtained with the nanocavity-based system. The reason for this is that the values obtained with the Quantaurus-QY system are influenced by absorbing but non-emitting molecules in the solution while the values obtained with 


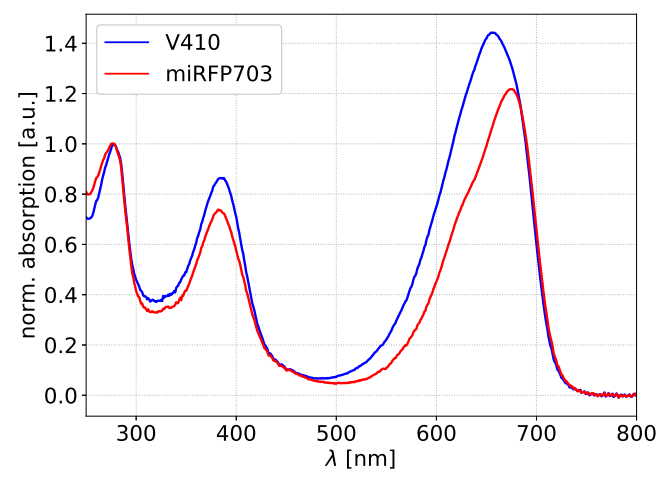

(A) Absorption spectra

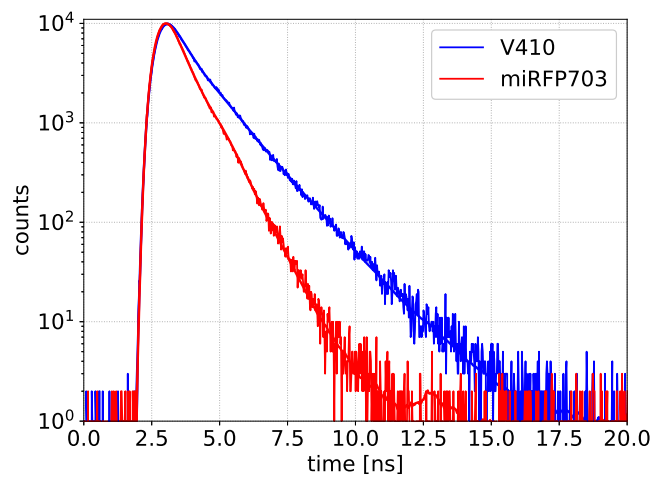

(C) Fluorescence lifetime decay histograms

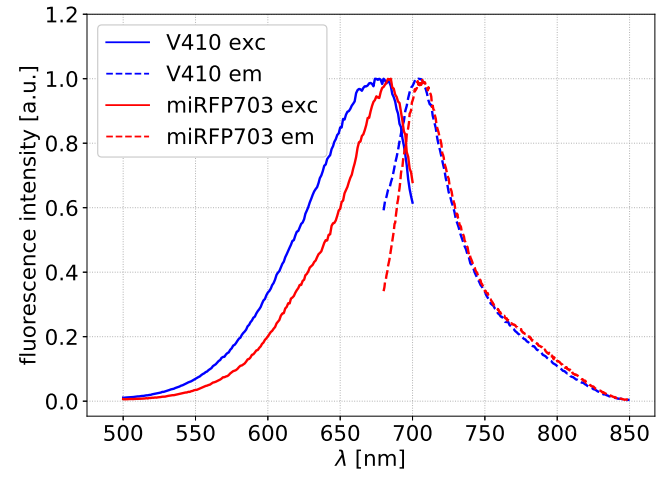

(B) Excitation and emission spectra

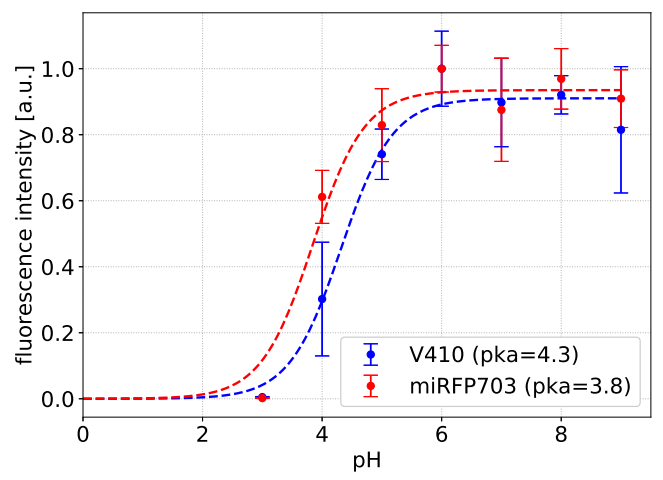

(D) $\mathrm{pH}$ dependend fluorescence intensity

FIGURE 3.7: Biophysical characterizations of V410. (A) Absorption spectra. (B) Excitation and emission spectra. (C) Fluorescence decay histograms. (D) $\mathrm{pH}$ depended fluorescence intensity. Together with V410, miRFP703 was measured for reference. For all measurements purified protein solutions were used.

the nanocavity-based system are not. Absorbing but non-emitting species could be, e.g., misfolded protein or free BV chromophore (see chapter 4.2). The molecular brightness is the product of fluorescence quantum yield and extinction coefficient. A molecular brightness of 24000 and 10900 was calculated for V410 and miRFP703, respectively (using the quantum yield values obtained by Dr. Alexey Chizhik).

\section{pH Stability of V410}

In mammalian cells, the $\mathrm{pH}$ value in different cell organelles varies from around 4.5 in lysosomes [2] to 8.0 in the mitochondrial matrix [96]. For a fluorescent protein to be useful as fusion tag for cellular structures in different organelles, the fluorescent protein must remain fluorescent over a wide range of $\mathrm{pH}$ values. The $\mathrm{pH}$ stability of V410 was determined by measuring the fluorescence signal of a purified protein solution at a concentration of $0.1 \mu \mathrm{g} / \mu \mathrm{l}$ in buffers with $\mathrm{pH}$ values ranging from 3 to 9. For comparison, the template miRFP703 was measured in the same way. The measurements were performed three times in replicates of $n=3$. The mean fluorescence signal of both proteins at different $\mathrm{pH}$ values can be seen in figure $3.7 \mathrm{~d}$. A monophasic model as described by equation (3.1) was fitted to the data points in 
Python using SciPy's curve_fit function [62].

$$
I_{670 \mathrm{~nm}}=o f f s e t+\frac{A}{1+10^{\left(p k_{a}-p H\right)}}
$$

This procedure yielded $p k_{a}$ values for V410 and miRFP703 of 4.3 and 3.8, respectively. Therefore, the protein variant V410 is highly fluorescent over the entire range of physiologically relevant $\mathrm{pH}$ values. This indicates its applicability as fusion tag in different cell organelles.

\section{Oligomeric State of V410}

The oligomeric state of a fluorescent protein is an important parameter since it determines the applicability of the protein as fusion tag. If a fluorescent marker protein forms dimers or higher oligomeric structures, tagging a protein of interest with it is likely to influence the localization of the protein of interest. Therefore, for labelling of structures with fluorescent molecules true monomeric fluorescent marker proteins are desirable.

The oligomeric state of the protein variant V410 was determined using size exclusion chromatography (SEC) and semi-native gel electrophoresis. For SEC, purified protein solution at a concentration of $10 \mu \mathrm{M}$ was loaded on the chromatography column. For semi-native gel electrophoresis, $4 \mu \mathrm{g}$ of purified protein was used. In both experiments the monomeric protein miRFP703 and the dimeric protein iRFP702 were used as controls. Figure 3.8a shows the elution profile of the size exclusion chromatography and figure $3.8 \mathrm{~b}$ shows the fluorescence image of the electrophoresis gel.

The elution profiles for the controls in figure 3.8a show a single peak around $16 \mathrm{ml}$ for the monomeric protein miRFP703 and a main peak for iRFP702 at an elution volume of $14 \mathrm{ml}$ with additional side peaks at smaller elution volumes. Since miRFP703 and iRFP702 are nearly identical in size, i.e., 315 amino acids and 311 amino acids, respectively, the elution profiles confirms that miRPF703 behaved like a monomer while iRFP702 had oligomerization tendencies at a concentration of $10 \mu \mathrm{M}$. The protein variant V410 had a similar elution profile as miRFP703 with a single peak at $16 \mathrm{ml}$. Therefore, V410 also behaved like a monomer at a concentration of $10 \mu \mathrm{M}$ in SEC. Also the semi-native gel electrophoresis confirmed the monomeric nature of V410 since only a single band was present at the same height as the single band of monomeric miRFP703. In contrast, for the oligomeric protein iRFP702 two bands were visible.

\section{Characterization of Brightness of V410 in Mammalian Cells}

For the directed evolution of V410 a bacterial expression system was used. However, the protein was developed for fluorescence imaging in mammalian cells. Therefore, the brightness of V410 in mammalian expression systems was characterized using flow cytometry. The experiment was performed with transiently transfected mammalian cells and with stable Bxb1 landing pad cell lines. The stable Bxb1 landing pad cell lines have the advantage that the expression level is much more homogeneous as compared to the transfected cells. For comparison of the brightness, the template miRFP703 was measured alongside V410. 

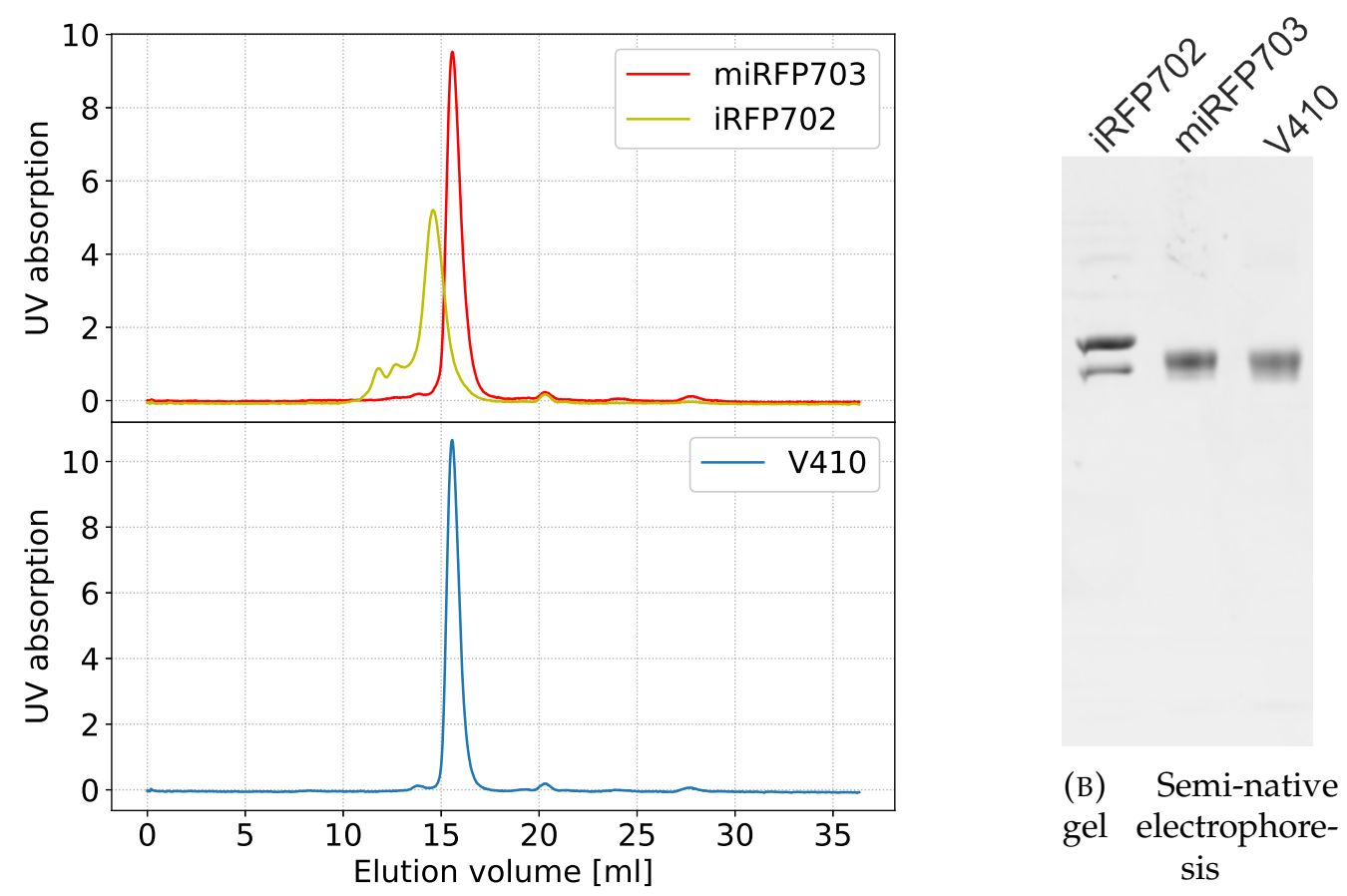

(A) Size exclusion chromatography elution profile

FIGURE 3.8: Characterization of oligomeric state of V410. (A) Elution profile of size exclusion chromatography of V410. (B) Semi-native gel electrophoresis of V410. For (A) and (B), miRFP703 and iRFP702 were measured alongside $\mathrm{V} 410$ for reference.

Figure E.1 shows the results of flow cytometry measurements of Bxb1 landing pad cell lines expressing the fusion constructs keratin-V410-P2A-mEGFP (P2690) and keratin-miRFP703-P2A-mEGFP (P2432), respectively. The cells were incubated in $0 \mu \mathrm{M}, 5 \mu \mathrm{M}$ or $25 \mu \mathrm{M}$ external BV for $2 \mathrm{~h}$ before the flow cytometry measurements. The mean brightness of the samples for the different detection channels is shown in figure 3.9 and in table 3.2.

\begin{tabular}{rrrrrr} 
& \multicolumn{2}{c}{$0 \mu \mathrm{M}$} & & \multicolumn{2}{c}{$5 \mu \mathrm{M}$} \\
\cline { 2 - 3 } \cline { 5 - 6 } & $690-720 \mathrm{~nm}$ & $720-766 \mathrm{~nm}$ & & $690-720 \mathrm{~nm}$ & $720-766 \mathrm{~nm}$ \\
\hline V410 & $506.00 \pm 0.48$ & $357.80 \pm 0.44$ & & $632.29 \pm 0.56$ & $483.11 \pm 0.505$ \\
miRFP703 & $603.61 \pm 0.62$ & $468.35 \pm 0.57$ & & $650.14 \pm 0.62$ & $507.91 \pm 0.57$ \\
\hline
\end{tabular}

\begin{tabular}{rcc} 
& \multicolumn{2}{c}{$25 \mu \mathrm{M}$} \\
\cline { 2 - 3 } & $690-720 \mathrm{~nm}$ & $720-766 \mathrm{~nm}$ \\
\hline V410 & $651.90 \pm 0.63$ & $501.55 \pm 0.55$ \\
miRFP703 & $649.78 \pm 0.64$ & $508.14 \pm 0.58$ \\
\hline
\end{tabular}

TABLE 3.2: Comparison of flow cytometry data of stable CRISPR cell lines 


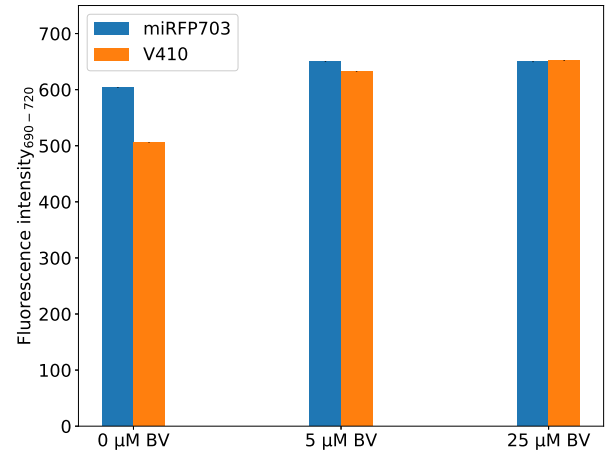

(A) Detection channel 690 to $720 \mathrm{~nm}$

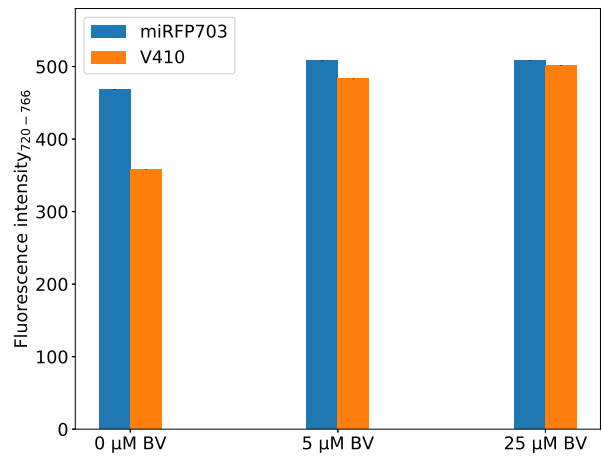

(B) Detection channel 720 to $766 \mathrm{~nm}$

FIGURE 3.9: Comparison of flow cytometry data of stable Bxb1 landing pad cell lines expressing the fusion constructs (P2690) keratin-V410-P2A-mEGFP (orange) and (P2432) keratin-miRFP703P2A-mEGFP (blue) after incubation with 0,5 and $25 \mu \mathrm{M}$ BV for $2 \mathrm{~h}$. (A) Fluorescence signal of cells in the detection window between 690 to $720 \mathrm{~nm}$. (B) Fluorescence signal of cells in the detection window between 720 to $766 \mathrm{~nm}$. Black bars indicate standard errors of the measurements. The measurements were performed in replicates of $\mathrm{n}=3$ with 10000 cells for each replicate.

Similarly, figure E.2 shows the results of flow cytometry measurements of HeLa cells transiently expressing V410-P2A-mEFGP and miRFP703-P2A-mEFGP, respectively. To account for different transfection efficiencies, the NIR fluorescence was normalized to the mEGFP fluorescence. $2 \mathrm{~h}$ before the measurements, the cells were incubated in $0 \mu \mathrm{M}, 5 \mu \mathrm{M}, 25 \mu \mathrm{M}$ or $100 \mu \mathrm{M}$ BV. The mean brightness of the samples is shown in figure 3.10 and in table 3.3.

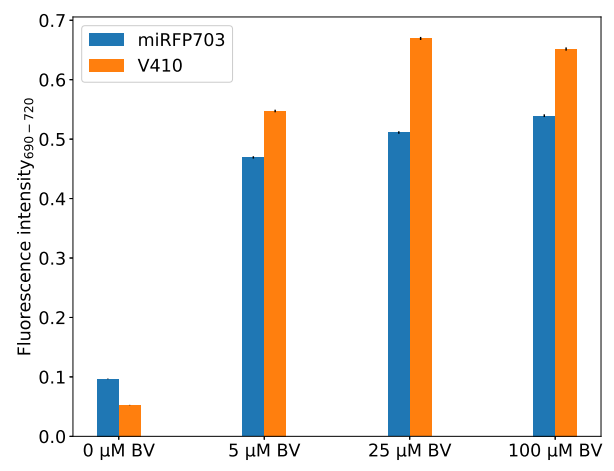

(A) Detection channel 690 to $720 \mathrm{~nm}$

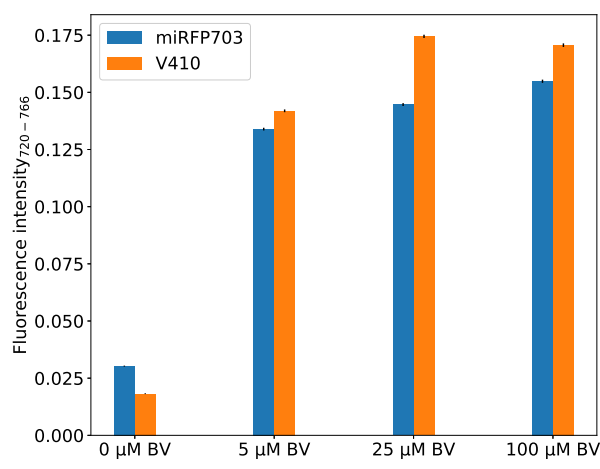

(B) Detection channel 720 to $766 \mathrm{~nm}$

FIGURE 3.10: Comparison of flow cytometry data of HeLa cells transiently expressing the fusion constructs (P3319) V410-P2A-mEGFP (orange) and (P3320) miRFP703-P2A-mEGFP (blue) after incubation with $0,5,25$ and $100 \mu \mathrm{M}$ BV for $2 \mathrm{~h}$. (A) Fluorescence signal of cells in the detection window between 690 to $720 \mathrm{~nm}$. (B) Fluorescence signal of cells in the detection window between 720 to $766 \mathrm{~nm}$. NIR fluorescence was normalized to mEGFP fluorescence to account for variations in transfection efficiency. Black bars indicate standard errors of the measurements. The measurements were performed in replicates of $n=5$ with 10000 cells for each replicate. 


\begin{tabular}{rrrrrr} 
& \multicolumn{2}{c}{$0 \mu \mathrm{M}$} & & \multicolumn{2}{c}{$5 \mu \mathrm{M}$} \\
\cline { 2 - 3 } \cline { 5 - 6 } miRFP703 & $690-720 \mathrm{~nm}$ & $720-766 \mathrm{~nm}$ & & $690-720 \mathrm{~nm}$ & $720-766 \mathrm{~nm}$ \\
\hline & $0.10 \pm 0.0005$ & $0.03 \pm 0.0002$ & & $0.47 \pm 0.0019$ & $0.13 \pm 0.0006$ \\
\cline { 5 - 6 } V410 & \multicolumn{2}{c}{$25 \mu \mathrm{M}$} & & \multicolumn{2}{c}{$100 \mu \mathrm{M}$} \\
\cline { 2 - 3 } \cline { 5 - 6 } & $690-720 \mathrm{~nm}$ & $720-766 \mathrm{~nm}$ & & $690-720 \mathrm{~nm}$ & $720-766 \mathrm{~nm}$ \\
\hline V410 & $0.67 \pm 0.0026$ & $0.17 \pm 0.0007$ & & $0.65 \pm 0.0027$ & $0.17 \pm 0.0008$ \\
miRFP703 & $0.51 \pm 0.0020$ & $0.14 \pm 0.0006$ & & $0.54 \pm 0.0023$ & $0.15 \pm 0.0007$ \\
\hline
\end{tabular}

TABLE 3.3: Comparison of flow cytometry data of transient expressions

The brightness distributions were very different between the stable and transient expressions. For the stable expression, the brightness of the cells followed approximately a normal distribution (as indicated by the dashed lines in figure E.1). In contrast, the brightness distribution of the transient expressions was clearly not normally distributed and varied across orders of magnitude. The reason for this is that transiently transfected cells take up different amounts of plasmid DNA during transfection.

For the stable expressions, miRFP703 was approximately $20 \%$ and $30 \%$ brighter than V410 in the respective detection channels without the addition of external BV. After incubation with $5 \mu \mathrm{M}$ BV for $2 \mathrm{~h}$, miRFP703 was still $3 \%$ and $5 \%$ brighter in the respective detection channels. At a BV concentration of $25 \mu \mathrm{M}$ V410 was approximately as bright as miRFP703. In contrast, for the transient expressions, miRFP703 was only brighter than V410 when no external BV was added. For $5 \mu \mathrm{M}, 25 \mu \mathrm{M}$ and $100 \mu \mathrm{M}$ external BV V410 was $17 \%$ and $8 \%, 31 \%$ and $21 \%, 20 \%$ and $13 \%$ brighter than miRFP703 in the respective detection channels. The maximum brightness of V410 in the transient expressions was reached after incubation with $25 \mu \mathrm{M} \mathrm{BV}$, while the maximum brightness of miRFP703 was reached after incubation with $100 \mu \mathrm{M}$ BV. For both proteins, the difference in brightness between incubation with $0 \mu \mathrm{M}$ and $5 \mu \mathrm{M}$ BV was bigger for the transient expression than for the stable expression.

To summarize the biophysical characterization of V410, the protein showed an excitation maximum at $677 \mathrm{~nm}$ and an emission maximum at $704 \mathrm{~nm}$ (see figure 3.7b). Therefore, excitation and emission maxima were located within the desired NIR spectral region. A fluorescence lifetime of $1120 \mathrm{ps}$ and a fluorescence quantum yield of $21 \%$ were measured for V410. In comparison, for miRFP703 a fluorescence lifetime of $690 \mathrm{ps}$ and a quantum yield of $12 \%$ were measured. Using this values, a molecular brightness of 24000 and 10900 was calculated for V410 and miRFP703, respectively. Furthermore, V410 showed a high $\mathrm{pH}$ stability with a $p k_{a}$ value of 4.3 (see figure 3.7d). The monomeric nature of V410 was confirmed in SEC (see figure 3.8a) and semi-native gel electrophoresis (see figure 3.8b). This suggests the applicability of V410 as a fusion tag in live-cell microscopy. In different mammalian expression systems the protein variant showed bright NIR fluorescence (see figure 3.9 and figure 3.10). However, especially without addition of external BV the higher quantum yield of V410 as compared to the template miRFP703 did not translate into likewise increased brightness in mammalian cells. Moreover, the difference in brightness 
between the addition of 0 and $5 \mu \mathrm{M}$ external BV was bigger for the transient expression than for the stable expression. This result suggests, that fluorescence imaging without addition of external BV should be performed using the stable expression system. For fluorescence imaging of transiently transfected mammalian cells, the addition of $25 \mu \mathrm{M}$ BV resulted into the brightest fluorescence. Therefore, whenever BV was added before imaging, a concentration of $25 \mu \mathrm{M}$ was used.

\subsection{Application of V410 in Live-Cell Imaging}

The protein variant V410 was developed for the application in NIR live-cell fluorescence microscopy. The following sections report on the results of the application of V410 in confocal, STED and FLIM imaging. If not stated differently, transfection of constructs for transient expression was performed approximately $24 \mathrm{~h}$ before imaging and approximately $2 \mathrm{~h}$ before imaging $25 \mu \mathrm{M}$ BV was added to the cells. Details on the exact imaging conditions used for the recording of the images shown in this chapter can be found in table D.1. All microscopy images depicted in this chapter are raw data if not stated otherwise.

\subsubsection{Confocal Imaging}

To investigate the usefulness of the protein variant V410 as fusion tag in live-cell fluorescence microscopy, confocal images of different cellular structures labeled with V410 were recorded. The cytoskeleton was targeted via $\beta$-actin (P3242), cytokeratin18 (P3241), vimentin (P2698) and Map2 (P2696) fusion constructs. The nuclear pore complex was targeted via a Nup50 fusion construct (P2697). Chromatin was stained via a H2B fusion construct (P3230). The outer mitochondrial membrane was targeted via a tomm20 fusion construct (P3279) and the centromeres were targeted via a fusion of V410 with CenpC1 (P3227). Additionally, the mitochondrial matrix (P3231) and peroxisomes (3229) were labeled by adding the respective import sequences $\mathrm{N}$ or C-terminally to the coding sequence of V410.

Aberrant cells with strong overexpression artefacts could be found for all transiently expressed fusion constructs. This was expected since the number of plasmid DNA a single cell takes up during transfection, and consequently the expression level, varies strongly between different cells. Exemplary raw images of cells with adequate expression levels for some fusion constructs are shown in figure 3.11.

The correct localisation of V410 in peroxisomes and the mitochondrial matrix demonstrated the applicability of V410 under basic $\mathrm{pH}$ conditions (see figure 3.11). Tightly packed and highly dynamic structures like the microtuble cytoskeleton did not show signs of impaired functionality caused by the indirect labelling with the V410-Map2 fusion construct (see figure 3.11 and figure 3.12). Likewise, H2B involved in the formation of tightly packed and strongly regulated chromatin structures could be labeled with the fluorescent protein without visibly altering cellular function or even triggering apoptosis (see figure 3.11).

Besides transient expression of fusion constructs, vimentin was tagged endogenously with V410 in HeLa cells via CRISPR/Cas-9 genome editing (P2691). This 

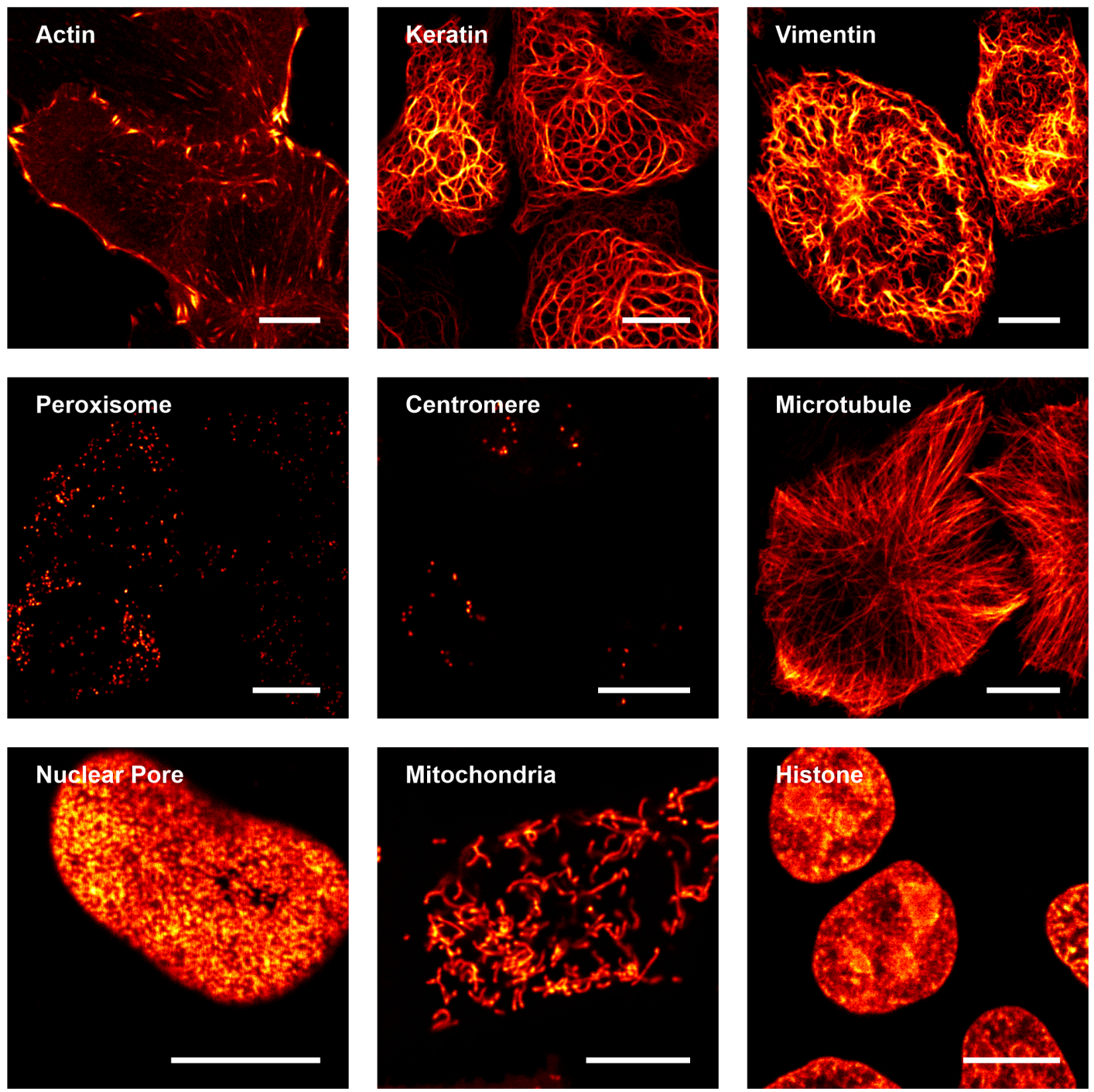

FIGURE 3.11: Confocal localisations of cellular structures with V410. HeLa cells were transiently transfected with the plasmid for microtubule localisation (P2696), nuclear pore localisation (P2697), vimentin localisation (P2698), centromere localisation (P3227), localisation in peroxisomes (P3229), histone localisation (P3230), localisation in the mitochondrial matrix (P3231), keratin localisation (P3241) and actin localisation (P3242), respectively. $25 \mu \mathrm{m} \mathrm{BV}$ was added $2 \mathrm{~h}$ before imaging. All images have different color maps. Scale bars: $10 \mu \mathrm{m}$.

allowed for expression of a V410-vimentin fusion construct close to endogenous expression levels. Additionally, a keratin-V410 fusion construct (P2690) was successfully integrated into a Bxb1 landing pad cell line [61], for stable expression of the fusion construct under the control of the CAG promoter in mammalian U2OS cells. The cells grew in cell culture over several generations without any sign of impaired proliferation or toxic effects due to the expression of the fusion constructs. In confocal imaging, cells of both cell lines showed homogeneous expression without aggregations or overexpression artefacts (see figure 3.16 and figure 3.19 for reference). The stable mammalian cell lines allowed for reproducible fluorescence measurements without perturbation of the cells by the harsh transfection procedure. 


\section{Confocal Movies}

To capture dynamic cellular processes, consecutive frames of the same region of interest were recorded. Figure 3.12a shows the frames 1, 250, 750 and 1000 of an exemplary series of 1000 confocal frames of HeLa cells transfected with a plasmid encoding for the expression of the fusion construct V410-Map2 (P2696). The mean brightness of every $10^{\text {th }}$ raw image is plotted in figure $3.12 \mathrm{~b}$.
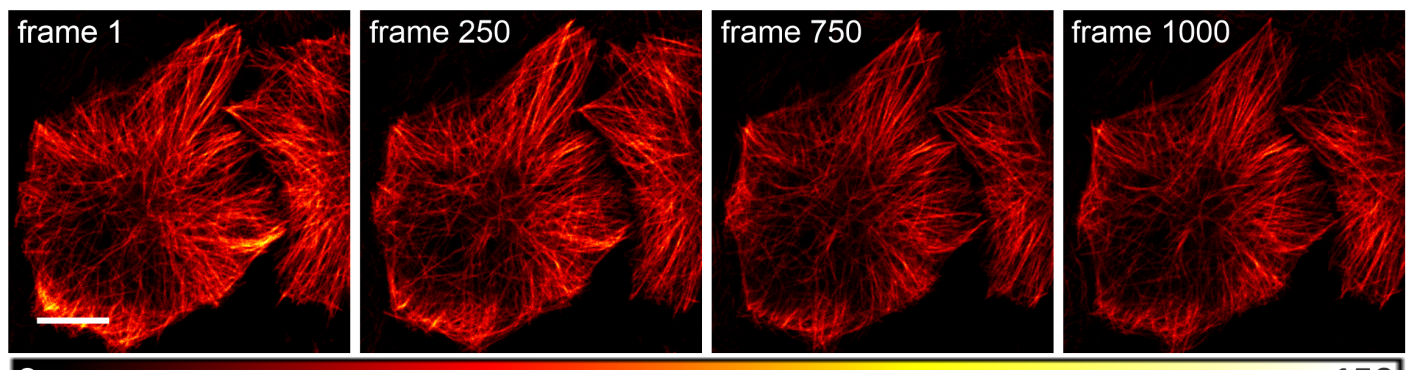

0

150

(A) Representative images from a series of 1000 consecutive confocal images

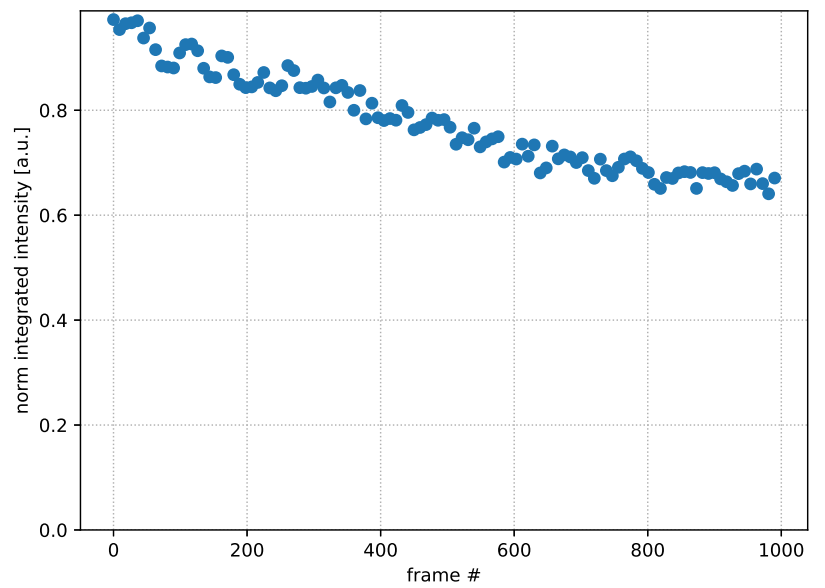

(B) Plot of normalized integrated intensity over 1000 consecutive confocal images

FIGURE 3.12: Recording of 1000 consecutive confocal images of HeLa transfected with the V410-Map2 fusion construct (P2696). (A) Representative images. (B) Plot of normalized integrated intensity. $25 \mu \mathrm{m}$ $\mathrm{BV}$ was added $2 \mathrm{~h}$ before imaging. Scale bar: $10 \mu \mathrm{m}$.

After 1000 consecutive confocal frames the mean brightness of the raw image was still above $60 \%$ of the initial brightness. This result demonstrates the high photo stability of V410 in NIR confocal live-cell imaging. Since the entire cell was imaged, also photo sensitive regions like the nucleus were exposed to high light doses. However, no signs of cellular response to photo toxic stress were noted by observing the fluorescent structures.

\subsubsection{Implementation of STED Imaging}

Since V410 showed correct localisations for various tagged proteins and high photostability in confocal imaging, the performance of the protein variant in live-cell STED microscopy was investigated next. An important parameter to optimize for 
STED microscopy is the utilized STED wavelength [137, 15]. For this, three experiments were performed to investigate the influence of the utilized STED wavelength on photo bleaching, reexcitation and STED efficiency for V410. For those experiments, a stable HeLa cell line expressing V410-keratin (P2690) was used.

\section{Correlation between Photo Bleaching and STED Wavelength}

Dynamic processes in living cells can only be observed if the recording of consecutive frames at the same position is possible before the fluorescent molecules in this region are destroyed by photo bleaching. To investigate the correlation between photo bleaching and STED wavelength, 20 consecutive STED frames were recorded. An excitation power of $20 \mu \mathrm{W}$ at $670 \mathrm{~nm}$ and a STED power of $45 \mathrm{~mW}$ (measured at the back aperture of the objective lens) for STED wavelengths between 790 to $840 \mathrm{~nm}$ were used. The measurements were performed with transiently transfected HeLa cells expressing a V410-Map2 fusion protein (P2696). The measurement was performed in replicates of $n=3$. The mean fluorescence intensity normalized to the first frame together with the standard deviation is plotted in figure 3.13a. The probability of photo bleaching is constant over consecutive time intervalls. Hence, photo bleaching of an assemble of molecules follows an exponential behaviour [7, 70, 106]. Because of this, an exponential model as described by equation (3.2) was fitted to the data points.

$$
I(t)=A \cdot e^{-K t}
$$

From the rate constant $\mathrm{K}$ the bleaching half time $t_{1 / 2}$ was calculated following equation (3.3).

$$
t_{1 / 2}=\frac{\ln (2)}{K}
$$

In figure $3.13 \mathrm{~b}$ the bleaching half time $t_{1 / 2}$ is plotted against the wavelength of the STED beam. The bleaching half time is lowest for a STED wavelength of $790 \mathrm{~nm}$ and highest for a STED wavelength of $840 \mathrm{~nm}$.

\section{Correlation between Reexcitation and STED Wavelength}

Another important consideration for choosing the optimal STED wavelength is the reexcitation behaviour of the fluorescent protein at that wavelength. A fluorescent protein in the electronic ground state $S_{0}$ cannot only be transferred to the first excited state $S_{1}$ by the excitation beam, but also by the STED beam. A high reexcitation cross section causes cycling of the fluorescent protein between the states $S_{0}$ and $S_{1}$ and consequently increased photo bleaching [91]. In order to measure the dependence of the reexcitation on the STED wavelength, the mean brightness of the same sample region was compared when excited with the excitation beam at $670 \mathrm{~nm}$ or the STED beam at different wavelengths and different STED powers. Powers were measured at the back aperture of the objective lens. Transiently transfected HeLa cells expressing a V410-Map2 fusion protein (P2696) were used to perform the measurements. For this experiment, the STED beam was used with a Gaussian beam profile instead of a donut shaped beam profile. The measurements were performed in replicates of $\mathrm{n}=3$. The ratio of the mean brightness when excited with $670 \mathrm{~nm} \overline{b r}_{\text {conf }}$ to the mean brightness when excited with different STED wavelengths $\overline{b r}_{\text {STED }}$ is plotted in figure 3.14a. The fluorescence emission intensity is proportional to the intensity of the 


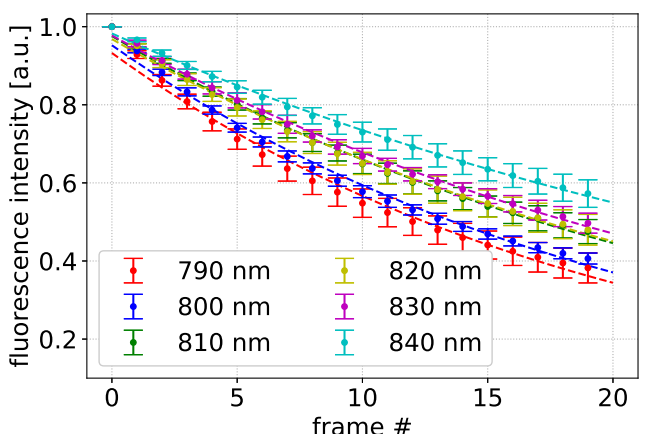

(A) Measurements of photo bleaching for different STED wavelengths

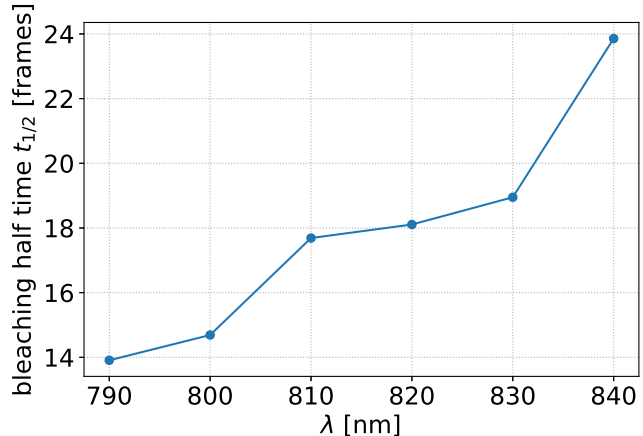

(B) Correlation between bleaching halftime and STED wavelength

FIGURE 3.13: Analysis of correlation between photo bleaching and STED wavelength. (A) Measurements of photo bleaching for STED wavelengths between 790 to $840 \mathrm{~nm}$. (B) Correlation between bleaching halftime and STED wavelength. Data were recorded using transiently transfected HeLa cells expressing a V410-Map2 fusion protein (P2696). $2 \mathrm{~h}$ before recording the data, $25 \mu \mathrm{M}$ BV was added to the cells. $20 \mu \mathrm{W}$ excitation power and $45 \mathrm{~mW}$ STED power, measured at the back aperture of the objective lens, were used to record the images.

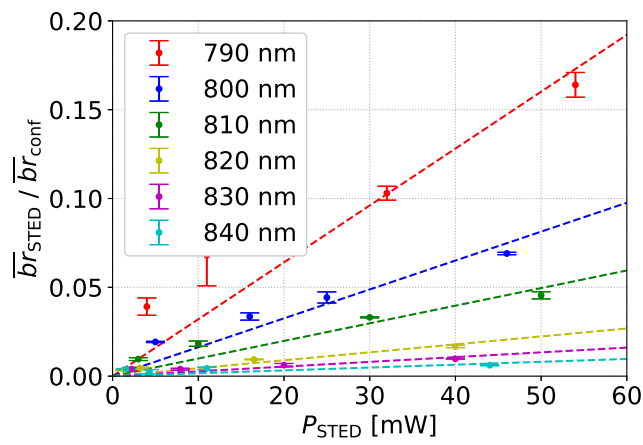

(A) Measurements of reexcitation for different STED wavelengths

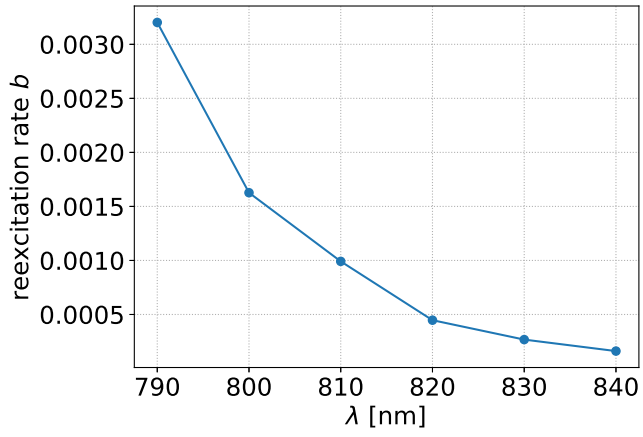

(B) Correlation between reexcitation and STED wavelength

FIGURE 3.14: Analysis of correlation between reexcitation and STED wavelength. (A) Measurements of reexcitation for wavelengths between 790 to $840 \mathrm{~nm}$. (B) Correlation between reexcitation and STED wavelength. Data were recorded using transiently transfected HeLa cells expressing a V410-Map2 fusion protein (P2696). $2 \mathrm{~h}$ before recording, the data $25 \mu \mathrm{M}$ BV was added to the cells. The STED pow-

ers were measured at the back aperture of the objective lens.

excitation light $[146,15]$. Therefore, a linear model as described by equation (3.4) was fitted to the data points.

$$
\overline{\overline{b r}}_{\mathrm{STED}}=b \cdot P_{\mathrm{STED}}
$$

In figure $3.14 \mathrm{~b}$, the reexcitation rate $b$ was plotted against the STED wavelength. The curve shows strongest reexcitation for a STED wavelength of $790 \mathrm{~nm}$ and the nearly 
no reexcitation for $840 \mathrm{~nm}$, resembling the absorption spectrum of V410 between 790 to $840 \mathrm{~nm}$.

\section{Correlation between STED Efficiency and STED Wavelength}

For a given STED beam intensity $I_{\text {STED }}$ the resolution in STED microscopy depends on the saturation intensity $I_{\text {sat }}$ (compare equation (1.7)). The saturation intensity $I_{\text {sat }}$, however, is a function of the STED wavelength. Consequently, an important consideration for choosing the ideal STED wavelength is the saturation intensity at this particular wavelength in order to maximize the resolution enhancement. To measure the STED efficiency, the mean brightness of a confocal image $\overline{b r}_{\text {conf }}$ was compared to the mean brightness of a STED image $\overline{b r}_{\text {STED }}$ of the same region for different STED wavelengths and STED intensities. Transiently transfected HeLa cells expressing a V410-keratin fusion protein (P3241) were used as a sample. For the confocal and STED images, an excitation power of $15 \mu \mathrm{W}$ at $670 \mathrm{~nm}$ (measured at the back aperture of the objective lens) was used. The STED beam had a Gaussian beam profile instead of a donut shaped beam profile. The measurements were performed in replicates of $\mathrm{n}=3$. In figures $3.15 \mathrm{a}$ to $3.15 \mathrm{f}$ the ratios $\overline{b r}_{\text {STED }} / \overline{b r}_{\text {conf }}$ were plotted for different STED wavelengths from 790 to $840 \mathrm{~nm}$.

An exponential model with an additional offset as described by equation (3.5) was fitted to the data points. This exponential model can be derived from a simplified two-level system where the additional offset term represents a confocal background [53].

$$
\frac{\overline{b r}_{\mathrm{STED}}}{\overline{b r}_{\mathrm{conf}}}=(1-\epsilon) \cdot e^{\left(-P_{\mathrm{STED}} / P_{\mathrm{sat}}\right)}+\epsilon
$$

In figure $3.15 \mathrm{~g}$ the saturation intensity $P_{\text {sat }}$ and the offset term $\epsilon$ were plotted against the STED wavelength. $P_{\text {sat }}$ exhibited a minimum at $790 \mathrm{~nm}$ and increased as the overlap between the spectrum of the STED beam and the emission spectrum of V410 decreased. An exception to this is the $P_{\text {sat }}$ value at $830 \mathrm{~nm}$ as it is slightly higher than the value for $840 \mathrm{~nm}$. The offset $\epsilon$ had a maximum value of approximately 0.126 at $790 \mathrm{~nm}$ and decreased monotonically to a value of 0.090 for $840 \mathrm{~nm}$.

To summarize, the bleaching half time $t_{1 / 2}$ increased with increasing STED wavelength from 790 to $840 \mathrm{~nm}$ (see figure 3.13b), while the reexcitation rate b decreased (see figure 3.14b). Measurements of the correlation between STED efficiency and STED wavelength showed an increase of the saturation power $P_{\text {sat }}$ and a decrease of the offset $\epsilon$ with increasing STED wavelength from 790 to $840 \mathrm{~nm}$ (see figure $3.15 \mathrm{~g}$ ). Additionally, technical considerations were taken into account when choosing the STED wavelength. A part of the STED beam was used for optical pumping of a photonic crystal fiber to produce the excitation light. The efficiency of this process and consequently the available excitation power was also influenced by the choice of the STED wavelength [32]. Based on this, the STED wavelength was set to $820 \mathrm{~nm}$ for the following STED experiments as a compromise between photo bleaching, reexcitation, STED efficiency, STED offset and efficiency of the optical pumping of the photonic crystal fiber. 


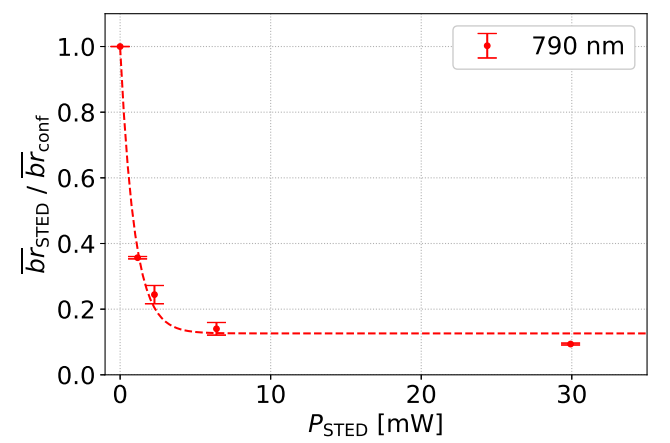

(A) Measurement of STED efficiency at $790 \mathrm{~nm}$

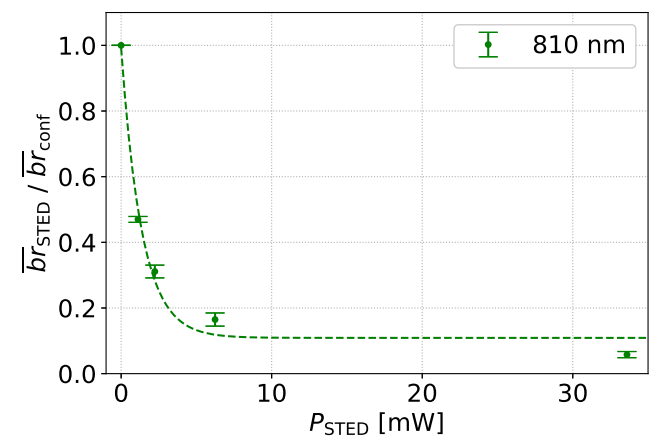

(C) Measurement of STED efficiency at $810 \mathrm{~nm}$

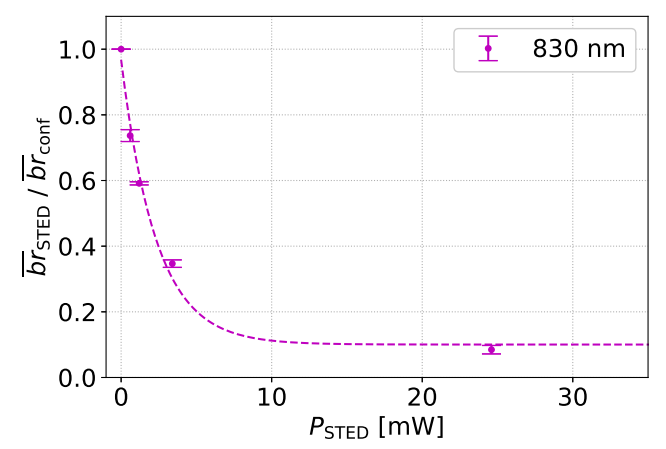

(E) Measurement of STED efficiency at $830 \mathrm{~nm}$

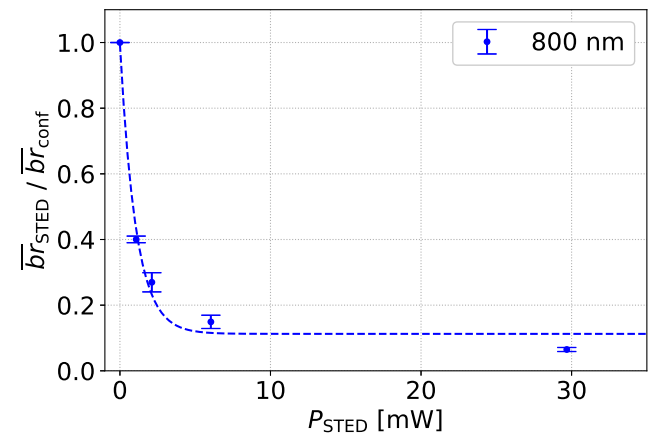

(B) Measurement of STED efficiency at $800 \mathrm{~nm}$

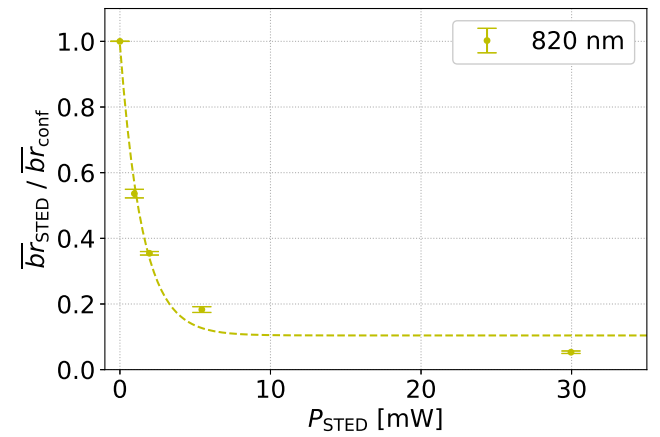

(D) Measurement of STED efficiency at $820 \mathrm{~nm}$

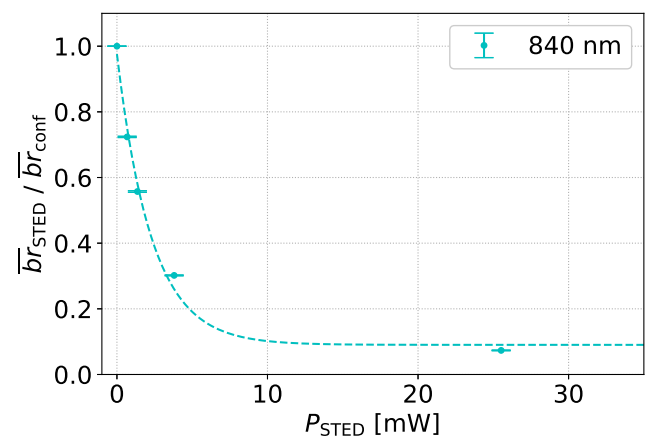

(F) Measurement of STED efficiency at $840 \mathrm{~nm}$ 


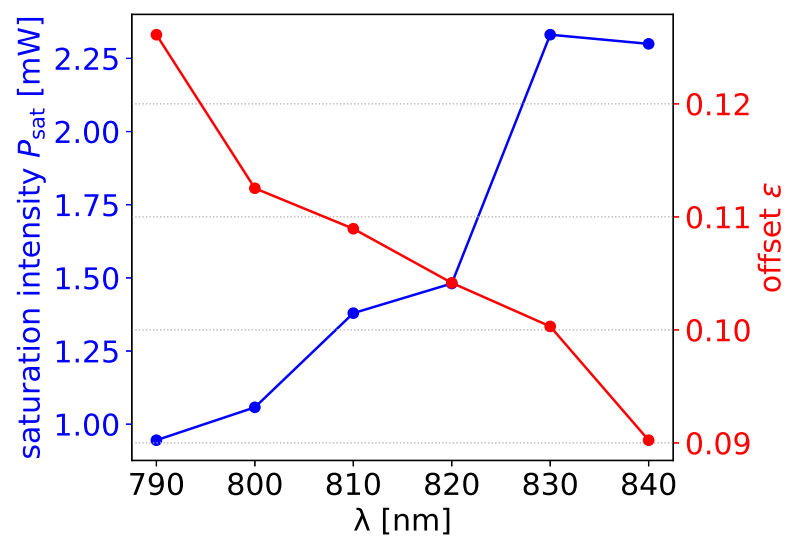

(G) Correlation between STED efficiency and STED wavelength

FIGURE 3.15: Analysis of correlation between STED efficiency and STED wavelength. (A) - (F) Measurements of STED efficiency for STED wavelenghts between 790 to $840 \mathrm{~nm}$. (G) Correlation between STED efficiency and STED wavelength. Data were recorded using transiently transfected HeLa cells expressing a V410-keratin fusion protein (P3241). $2 \mathrm{~h}$ before recording the data $25 \mu \mathrm{M}$ BV was added to the cells. An excitation power of $15 \mu \mathrm{W}$ was used for confocal and STED images. The powers were measured at the back aperture of the objective lens.

\subsubsection{STED Imaging}

After the best depletion wavelength for STED imaging of protein variant V410 has been established in section 3.4.2, section 3.4.3 reports on the results of live-cell STED microscopy of different cellular structures labeled with V410.

First, STED imaging of living CRISPR/Cas-9 genome edited U2OS cells stably expressing a vimentin-V410 fusion protein (P2691) was performed. Due to the physiological expression level of the fusion construct, a thin filamentous network spanned through the entire cell. Because of this, the stable CRISPR cell line was a well suited sample for evaluating the achievable resolution without impairment by overexpression artefacts. Figure 3.16a shows the confocal and STED overview images of a hole U2OS cell next to the zoomed confocal and STED images of the marked region.

To determine the resolution of the images, 14 line profiles were drawn as indicated in the images. To account for random fluctuations in the line profiles due to noise, a pixel averaging of three pixels was used. This means, the line profiles shown here are actually the average line profiles of three neighboring and parallel line profiles. A Lorentzian model as described by equation (2.1) was fitted to the line profiles. Representative line profiles are shown in figures 3.16b to 3.16e (for all line profiles see figure D.3).

The median of the FWHM of the confocal line profiles was $306 \mathrm{~nm}$. $268 \mathrm{~nm}$ was the lowest FWHM and $346 \mathrm{~nm}$ was the highest FWHM that was measured. The measured values are higher than the theoretical resolution of an ideal confocal microscope (see equation $(3.6)[25,26])$. The reasons for this discrepancy are, i.a., the 

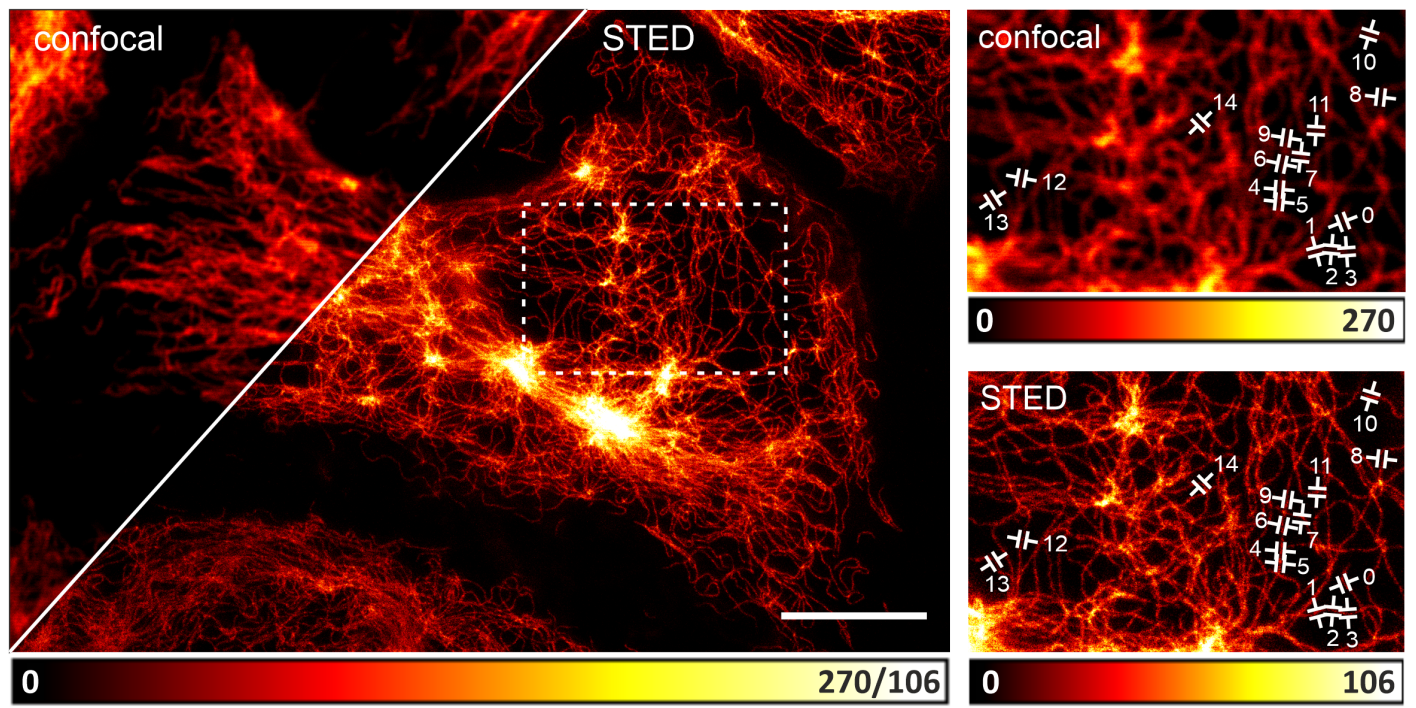

(A) Confocal and STED images

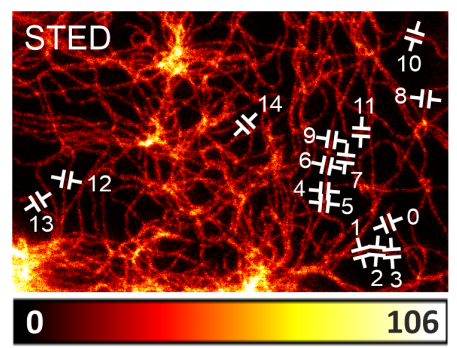

(A) Confocal and STED images

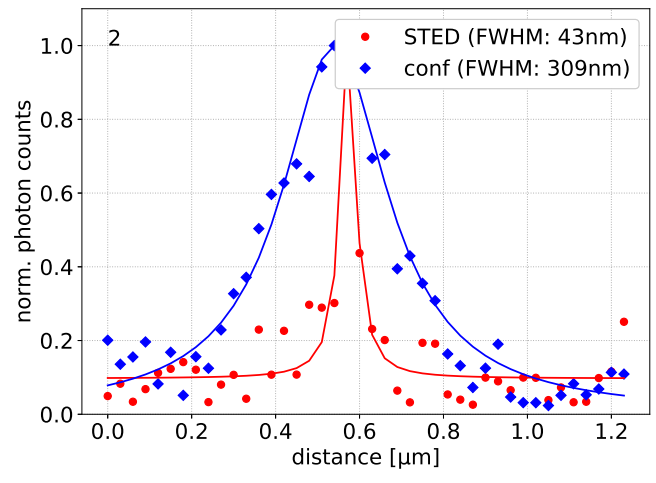

(B) Line profile 2

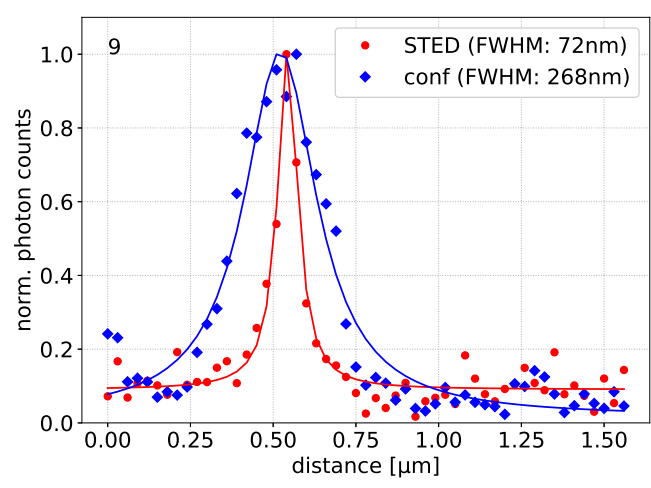

(D) Line profile 11

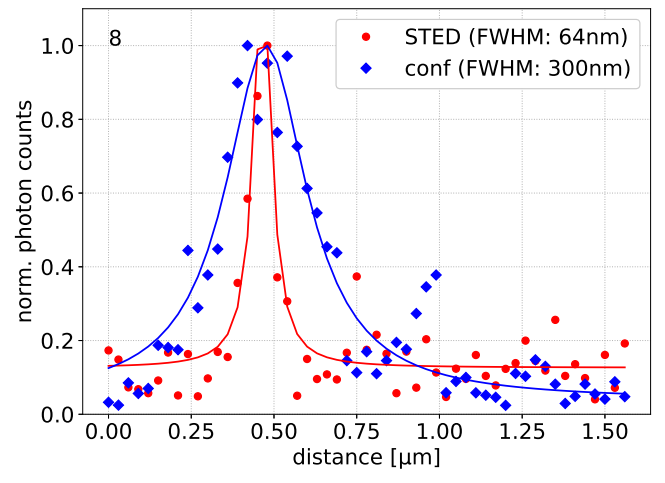

(C) Line profile 9

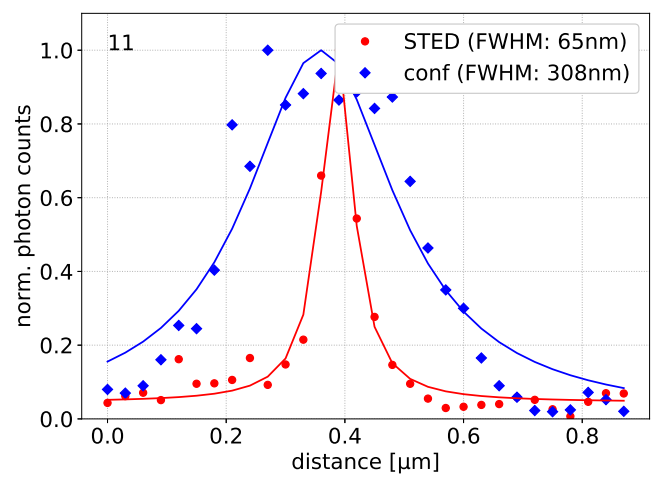

(E) Line profile 13

FIGURE 3.16: Confocal and STED recordings of genome edited U2OS CRISPR cell line expressing vimentin-V410 (P2691). (A) Overview and zoomed confocal and STED images. (B) - (E) Confocal (blue) and STED (red) line profiles as indicated in (A). $25 \mu \mathrm{m} \mathrm{BV}$ was added $2 \mathrm{~h}$ before imaging. Scale bar: $10 \mu \mathrm{m}$. 

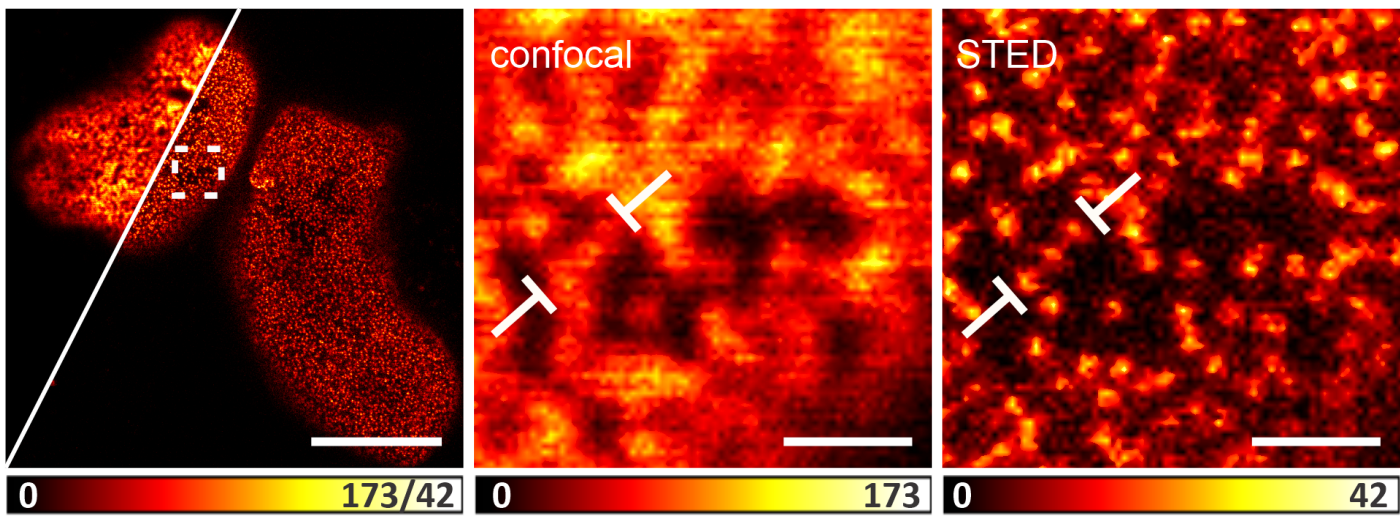

0

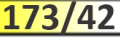

\section{0}

1730

(A) Confocal and STED images

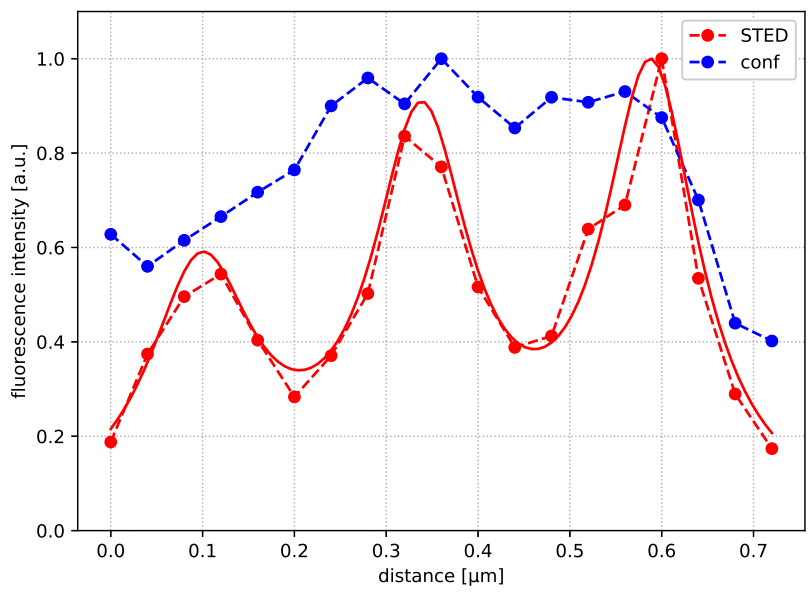

(B) Confocal and STED line profile

FIGURE 3.17: Confocal and STED recordings of HeLa cells transiently expressing V410-Nup50 (P2697). (A) Overview and zoomed confocal and STED images. (B) Confocal (blue) and STED (red) line profiles as indicated in (A). $25 \mu \mathrm{m}$ BV was added $2 \mathrm{~h}$ before imaging. Scale bars:

$10 \mu \mathrm{m}$ (overview image), $1 \mu \mathrm{m}$ (zoomed image).

finite pinhole size (equation (3.6) assumes an infinitesimal small pinhole size) and optical abberations.

$$
F W H M_{\text {conf }}=\frac{0.37 \cdot\left(\frac{670 \mathrm{~nm}+700 \mathrm{~nm}}{2}\right)}{1.4}=181 \mathrm{~nm}
$$

For STED, the median FWHM was $83 \mathrm{~nm}$, the lowest FWHM was $43 \mathrm{~nm}$ and the highest FWHM was $98 \mathrm{~nm}$. Hence, the 2D resolution in the STED image is improved by a factor of 3 to 4 as compared to the confocal image.

Next, transiently transfected HeLa cells were imaged with STED microscopy. Figure 3.17a shows the exemplary confocal and STED overview images of two HeLa cells transiently expressing a V410-Nup50 fusion construct (P2697) next to the zoomed confocal and STED images of the indicated region. The line profiles over three nuclear pore complexes indicated in the zoomed images are plotted in figure $3.17 \mathrm{~b}$. The sum of three Lorentzian functions centered at different coordinates was fitted to the line profile of the STED image. The comparison between the confocal and STED 

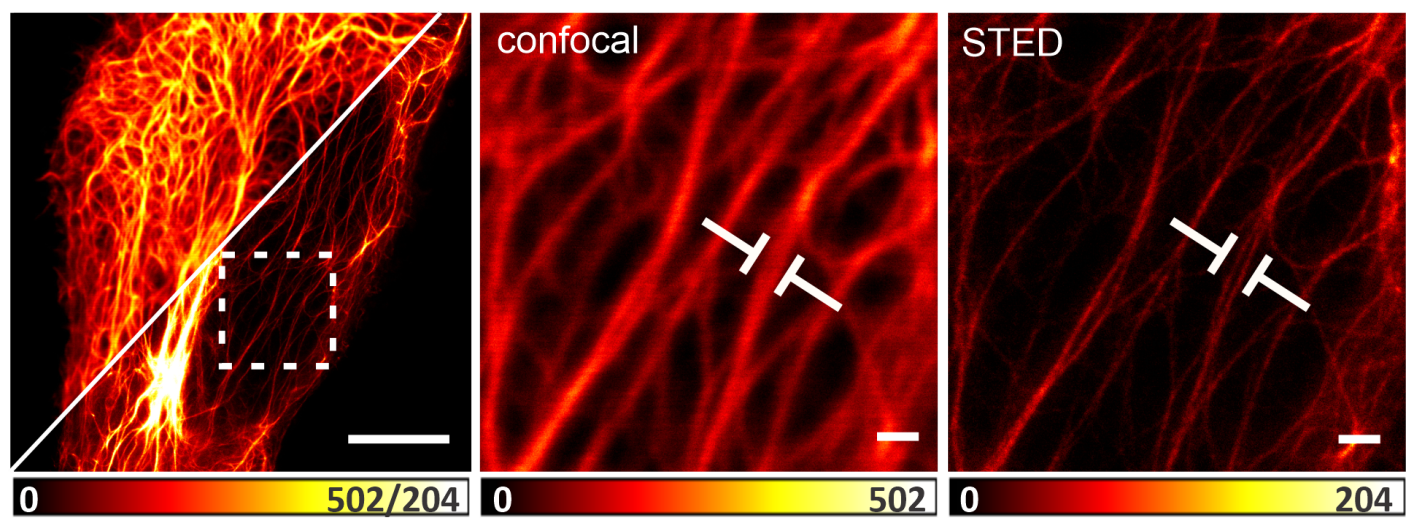

(A) Confocal and STED images

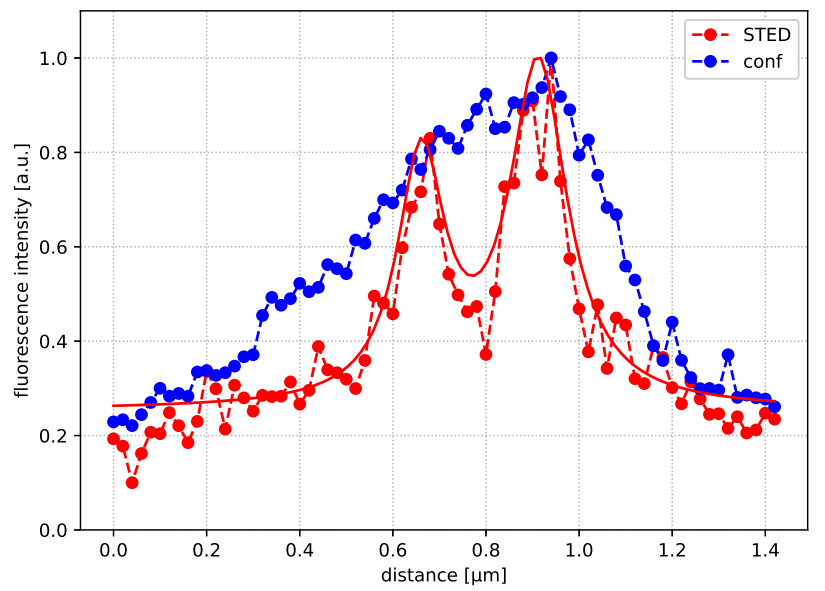

(в) Confocal and STED line profile

FIGURE 3.18: Confocal and STED recordings of HeLa cells transiently expressing V410-keratin (P3241). (A) Overview and zoomed confocal and STED images. (B) Confocal (blue) and STED (red) line profiles as indicated in (A). $25 \mu \mathrm{m}$ BV was added $2 \mathrm{~h}$ before imaging. Scale bars:

$10 \mu \mathrm{m}$ (overview image), $1 \mu \mathrm{m}$ (zoomed image).

line profiles reveals the resolution enhancement achieved with STED microscopy. In the confocal image, the neighbouring nuclear pore complexes could not be distinguished while STED microscopy clearly resolved three distinct complexes. The central intensity maxima of the complexes are approximately $240 \mathrm{~nm}$ apart. Figure 3.18a shows an exemplary overview and zoomed images of HeLa cells transiently expressing V410 tagged to keratin (P3241). Again, line profiles were taken at the indicated regions to illustrate the resolution improvement of STED imaging compared to confocal imaging (see figure 3.18b). Due to the limited resolution only a single filament was visible at the indicated position in confocal microscopy. The improved resolution of STED microscopy revealed two filaments with a distance of $250 \mathrm{~nm}$ from peak to peak.

The desired application for NIR fluorescent proteins in the future is deep-tissue fluorescence imaging of living mammalian organisms. For this, the physiological BV levels in living mammalian cells must be sufficient for the fluorescent protein. To examine if V410 can be imaged in mammalian cells with physiological BV levels, confocal and STED images of stable Bxb1 landing pad HeLa cells expressing a 

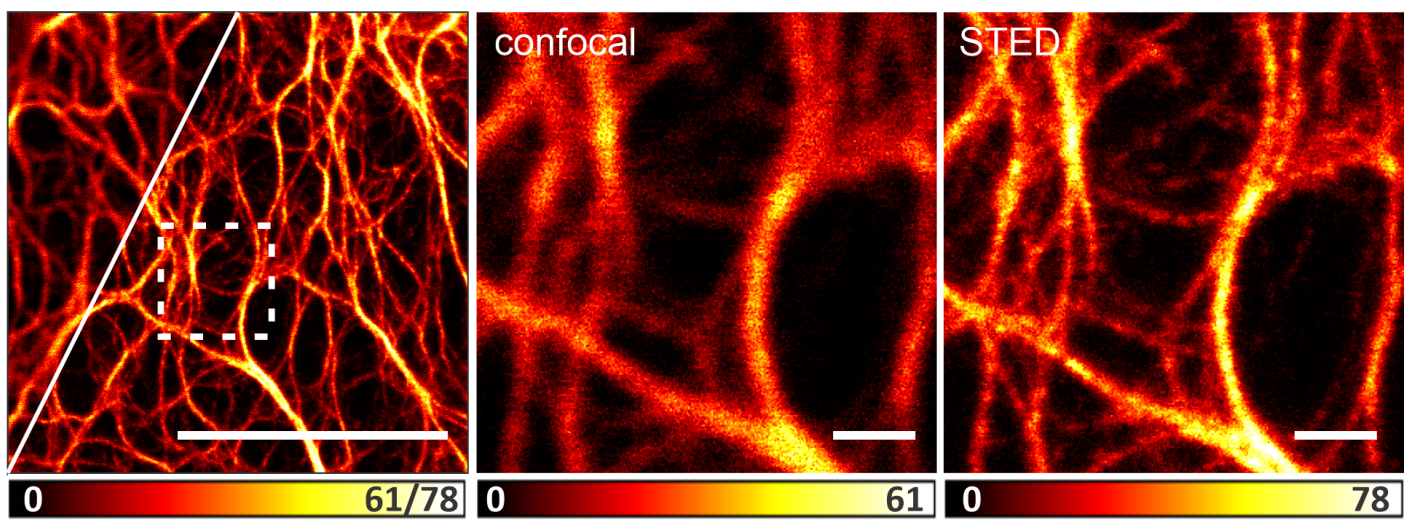

FIGURE 3.19: Confocal and STED recordings of stable Bxb1 landing pad cell line expressing keratin-V410 (P2690). No BV was added before imaging. Scale bars: $10 \mu \mathrm{m}$ (overview image), $1 \mu \mathrm{m}$ (zoomed image).

keratin-V410 fusion protein were recorded without the addition of $25 \mu \mathrm{M}$ BV before imaging. The resulting images are shown in figure 3.19. The keratin structures were much dimer as compared to the situation with external supply of $25 \mu \mathrm{M}$ BV before imaging. Additionally, the labelling density seemed worse. This means the intensity along filaments fluctuated more and was not as homogeneous compared to a sample incubated with external BV before imaging. However, by adjusting the imaging parameters (e.g. increasing the pixel dwell time) it was possible to capture the keratin network of a HeLa cell with confocal and STED imaging without the supply of external BV.

In summary, live-cell STED nanoscopy of transiently transfected mammalian cells and stable mammalian cell lines with and without addition of external BV was performed. Using endogenously labeled U2OS cells expressing a vimentin-V410 fusion protein, resolutions down to $43 \mathrm{~nm}$ were measured. Next, consecutive STED images were recorded to capture dynamic cellular processes of living mammalian cells with increased resolution.

\section{STED Movies}

As stated earlier, dynamic cellular processes can only be captured by fluorescence microscopy if the recording of consecutive images of the same region is possible. For confocal microscopy, it could be demonstrated that after 1000 consecutive images of mammalian cells transiently expressing V410 tagged to Map2 only approximately $40 \%$ of the initial fluorescence signal was lost due to photo bleaching (see figure 3.12b). For STED microscopy, the effect of photo bleaching is in general even more pronounced compared to confocal microscopy. To evaluate this, HeLa cells were transiently transfected with a plasmid for the expression of V410-Map2 (P2696). After incubation for $2 \mathrm{~h}$ with $25 \mu \mathrm{M}$ BV, STED movies were recorded. Figure 3.20a shows the frames 1, 25, 75 and 100 of an exemplary recording of 100 consecutive STED frames. The normalized integrated intensity is plotted in figure 3.20c. After 100 STED frames approximately $50 \%$ of the initial fluorescence signal of the image was lost due to photo bleaching. To correct for the effect of photo bleaching, the bleaching correction algorithm of histogram matching was applied using the BleachCorrector plugin from the software Fiji $[84,116]$. Figure $3.20 \mathrm{~b}$ shows the 
corrected frames 1, 25, 75 and 100. Note, that after applying histogram matching the color of the pixels does not reflect the actual photon counts in that pixel anymore. Therefore, quantitative analysis of the corrected images is not possible. After applying bleaching correction fine filaments were clearly visible even after 100 consecutive STED frames.

\subsubsection{Fluorescence Lifetime Imaging}

For many applications in cellular research, it is not sufficient to only have information about the distribution and abundance of a single fluorescently labeled protein. To learn about the interplay between different proteins, it is necessary to be able to label and detect two or more proteins of interest at the same time. One way to do this, is the labelling of different proteins with spectrally distinct fluorescent labels. However, in general this approach requires at least one additional excitation light source, exposing the biological sample to additional photo stress and rendering imaging entirely within the NIR spectral region difficult. In this section, an alternative approach was used, namely fluorescence lifetime imaging (FLIM), where the labels are not spectrally distinct but have distinct fluorescence lifetimes. This enables two color imaging with only one excitation source in the NIR spectral region.

In practice, two-color FLIM images were recorded using a Bxb1 landing pad HeLa cell line stably expressing keratin-miRFP703 (P2432) $\left(\tau_{\text {miRFP703 }}=690 \mathrm{ps}\right)$ that was transfected with a plasmid for transient expression of tomm20-V410 (P3279) $\left(\tau_{\mathrm{V} 410}=1120 \mathrm{ps}\right)$. The exact imaging parameters are listed in table D.1. For recording of reference lifetime histograms, the stable cell line expressing keratin-miRFP703 alone and normal HeLa cells only transiently expressing tomm20-V410 were used, respectively. Subsequently, the reference histograms were accumulated by summing the decay histograms in all pixels above an intensity threshold for the respective FLIM images.The FLIM images used for the accumulation of reference histograms are shown in figure 3.21a and the accumulated reference histograms are shown in figure $3.21 \mathrm{~b}$.

\section{Decomposition Methods}

Two different methods have been implemented in Python for decomposing the recorded two-color FLIM images into distinct channels for the respective fluorescent protein based on their characteristic fluorescence decay. Both methods assume that the measured fluorescence decay $\overrightarrow{b_{n}}$ in every pixel of the FLIM image is a linear combination of the reference decays $\overrightarrow{r_{1}}$ and $\overrightarrow{r_{2}}$ plus noise $\overrightarrow{\epsilon_{n}}$ (with zero mean) as described by equation (3.7) with $n$ being the pixel index and $y$ being the number of bins in the decay histograms.

$$
a_{1 n} \underbrace{\left[\begin{array}{c}
r_{11} \\
r_{12} \\
\vdots \\
r_{1 y}
\end{array}\right]}_{\overrightarrow{r_{1}}}+a_{2 n} \underbrace{\left[\begin{array}{c}
r_{21} \\
r_{22} \\
\vdots \\
r_{2 y}
\end{array}\right]}_{\overrightarrow{r_{2}}}+\underbrace{\left[\begin{array}{c}
\epsilon_{n 1} \\
\epsilon_{n 2} \\
\vdots \\
\epsilon_{n y}
\end{array}\right]}_{\overrightarrow{\epsilon_{n}}}=\overrightarrow{\lambda_{n}}+\overrightarrow{\epsilon_{n}}=\underbrace{\left[\begin{array}{c}
b_{n 1} \\
b_{n 2} \\
\vdots \\
b_{n y}
\end{array}\right]}_{\overrightarrow{b_{n}}}
$$

The first method finds the least squares solution of matrix equation (3.8) and is based on a publication from Enderlein et al. [37]. Matrix $R$ is composed of the reference decays $\overrightarrow{r_{1}}$ and $\overrightarrow{r_{2}}$ as column vectors. Matrix $A$ is composed of the unknown coefficients 

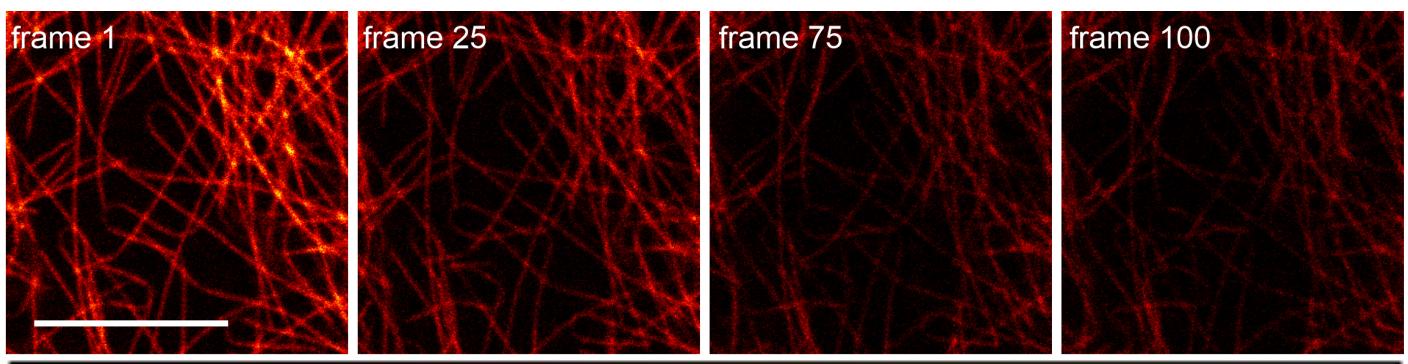

0

(A) Representative images from a series of 100 consecutive STED images
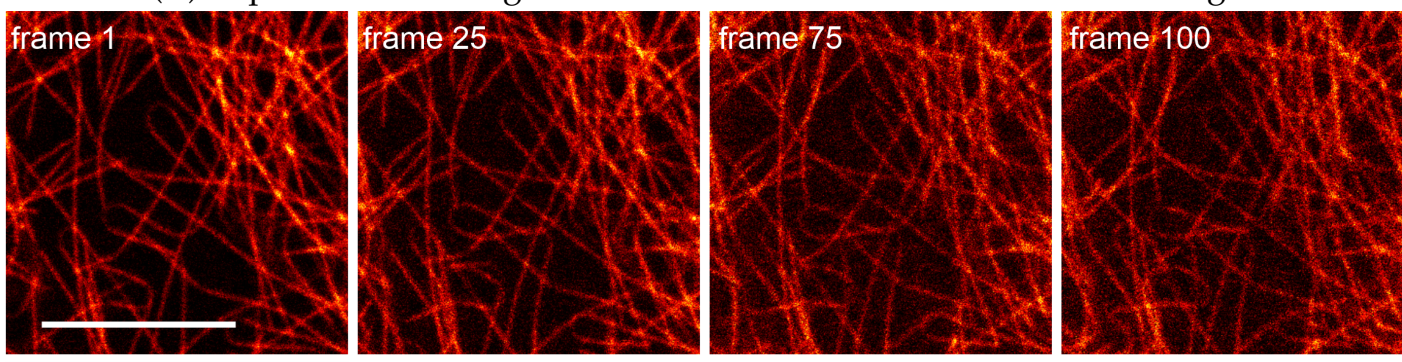

O

(B) Representative images from a series of 100 consecutive STED images with applied bleaching correction

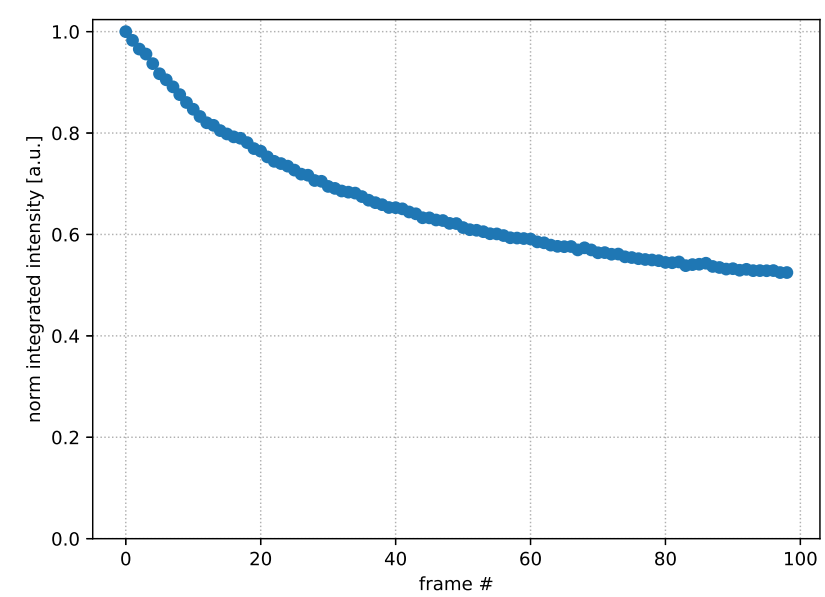

(C) Plot of normalized integrated intensity over 100 consecutive STED images

FIGURE 3.20: Recording of 100 consecutive STED images of HeLa transiently expressing V410-Map2 (P2696). (A) Representative images. (B) Representative images with applied bleaching correction (histogram matching) with Fiji [84, 116] (After bleaching correction color does not reflect actual photon counts). (C) Plot of normalized integrated intensity. $25 \mu \mathrm{m} \mathrm{BV}$ was added $2 \mathrm{~h}$ before imaging. Scale bar: $10 \mu \mathrm{m}$. 


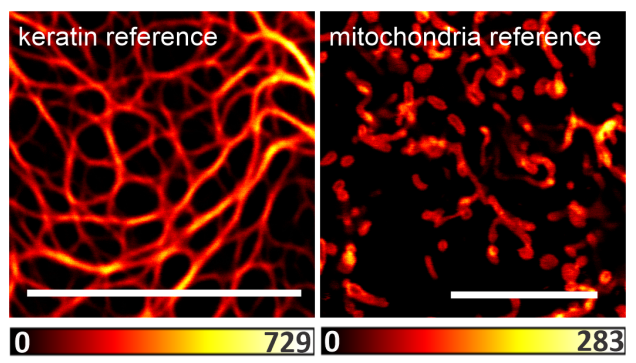

(A) Reference images

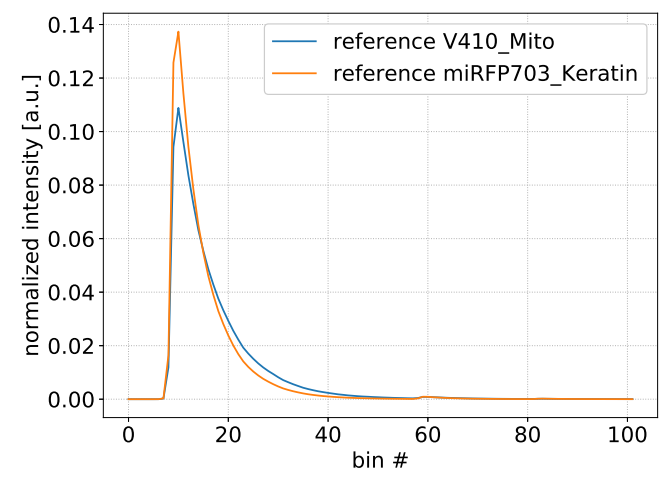

(B) Reference histograms

FIGURE 3.21: Reference images and histograms for FLIM. (A) Reference FLIM images of stable keratin-miRPF703 cell line (P2432) and HeLa cells transiently expressing tomm20-V410 (P3279). (B) Reference histograms accumulated from reference FLIM images. The reference histograms are normalized to an integral of 1 . Scale bars: $10 \mu \mathrm{m}$.

$a_{1 n}$ for the first reference decay and $a_{2 n}$ for the second reference decay for all $x$ pixel. Matrix $B$ is composed of the measured decay histograms $\overrightarrow{b_{n}}$ of the FLIM image as column vectors. A schematic illustration of equation (3.8) can be found in figure C.1.

$$
\underbrace{\left[\begin{array}{cc}
r_{11} & r_{21} \\
r_{12} & r_{22} \\
\vdots & \vdots \\
r_{1 y} & r_{2 y}
\end{array}\right]}_{R} \underbrace{\left[\begin{array}{cccc}
a_{11} & a_{12} & \cdots & a_{1 x} \\
a_{21} & a_{22} & \cdots & a_{2 x}
\end{array}\right]}_{A}=\underbrace{\left[\begin{array}{cccc}
b_{11} & b_{21} & \cdots & b_{x 1} \\
b_{12} & b_{22} & \cdots & b_{x 2} \\
\vdots & \vdots & \ddots & \vdots \\
b_{1 y} & b_{2 y} & \cdots & b_{x y}
\end{array}\right]}_{B}
$$

The least squares solution of equation (3.8) is given by equation 3.9.

$$
A=\left(R^{T} R\right)^{-1} R^{T} B
$$

The second method aims to find the most likely linear combination of reference decays $\overrightarrow{\lambda_{n}}$ for pixel $n$ given the measured decay $\overrightarrow{b_{n}}$ on the basis of Poisson statistics. This approach is reasonable because the main source of noise in fluorescence lifetime decay histograms is Poisson noise (i.e. shot noise) due to the quantum mechanical nature of fluorescence. Equation (3.10) shows the probability mass function describing this process with $b_{n m}$ being the number of measured photons in the $m$ th bin of the $n$th pixel of the FLIM image and $\lambda_{n m}$ being the number of expected photons in the $m$ th bin for the $n$th pixel of the model function.

$$
f\left(b_{n m} \mid \lambda_{n m}\right)=\frac{\lambda_{n m}^{b_{n m}}}{b_{n m} !} e^{-\lambda_{n m}}, \quad b_{n m}=0,1,2, \ldots, \infty
$$

By applying the natural logarithm to both sides of equation (3.10) it can be rewritten as equation (3.11).

$$
\ln (f)=b_{n m} \ln \left(\lambda_{n m}\right)-\lambda_{n m}-\ln \left(b_{n m} !\right)
$$

To maximize the likelihood based on the entire decay curve, summation over all $\mathrm{m}$ bins has to be performed as described by equation (3.15). 


$$
\sum_{m=0}^{y} \ln (f)=\sum_{m=0}^{y} b_{n m} \ln \left(\lambda_{n m}\right)-\lambda_{n m}-\ln \left(b_{n m} !\right)
$$

Under the assumption that $\sum_{m=0}^{y} \lambda_{n m} \equiv \sum_{m=0}^{y} b_{n m}\left(\overrightarrow{\epsilon_{n}}\right.$ has a mean of zero) the terms $-\lambda_{n m}$ and $-\ln \left(b_{n m} !\right)$ become constant and can be ignored for the maximization of the probability. This simplifies equation (3.15) to equation (3.13). From the assumption, it can additionally be derived that the sum of the amplitudes $a_{1 n}$ and $a_{2 n}$ is equal to the sum of counts in the $n$th pixel of the FLIM image $\sum_{m=0}^{y} \lambda_{n m}$ if the reference decays $\overrightarrow{r_{1}}$ and $\overrightarrow{r_{2}}$ are normalized so that the integral of each histogram equals 1 . This allows to write the model function as described by equation (3.14).

$$
\begin{gathered}
\sum_{m=0}^{y} \ln (f)=\sum_{m=0}^{y} b_{n m} \ln \left(\lambda_{n m}\right)=\overrightarrow{b_{n}} \cdot \ln \left(\overrightarrow{\lambda_{n}}\right) \\
\overrightarrow{\lambda_{n}}=a_{1 n} \overrightarrow{r_{1}}+\left(\sum_{m=0}^{y} b_{n m}-a_{1 n}\right) \overrightarrow{r_{2}}
\end{gathered}
$$

Therefore, the optimization problem was finding the $a_{1 n}$ for every pixel $\mathrm{n}$ in the FLIM image that maximizes equation (3.13). In practice, instead of maximizing equation (3.13) both sides of the equation were multiplied by -1 and minimized in Python using SciPy's minimize function [62] (see equation (3.15)).

$$
\underset{a_{1 n}}{\operatorname{argmin}}\left(-\overrightarrow{b_{n}} \bullet \ln \left(a_{1 n} \overrightarrow{r_{1}}+\left(\sum_{m=0}^{y} b_{n m}-a_{1 n}\right) \overrightarrow{r_{2}}\right)\right)
$$

\section{Evaluation of Decomposition Methods}

To evaluate the performance of the different decomposition approaches, two evaluation experiments were performed. In the first experiment, both approaches were evaluated on simulated data. For this, 500 fluorescence decays with different noiseto-signal levels were simulated by adding the normalized reference histograms with equal amplitudes, scaling them with a factor and adding shot noise to the resulting histograms. Both approaches were used to decompose the simulated decays. The standard deviations of the ratios $a_{1} / a_{2}$ from 100 decompositions for every noiseto-signal level for both decomposition approaches are plotted in figure 3.22. For all noise-to-signal levels the maximum likelihood method outperformed the least squares method. While at low noise-to-signal levels the difference in performance between maximum likelihood and least squares method was only little, the performance gap became bigger for higher noise-to-signal levels.

For the second evaluation experiment, both reference images from figure 3.21 were decomposed into two separate channels with either approach. Since only one fluorescent protein was present in the respective reference image, an ideal decomposition of the reference images would reconstruct the initial image in one channel while the second channel would be empty. The results of the decompositions are shown in figure D.4 for the maximum likelihood algorithm and figure D.5 for the least squares algorithm. The count ratios for the different channels from the decompositions of the reference images for both decomposition approaches are plotted in figure D.6. Again, the maximum likelihood method performed better in the experiment than the least squares method as less photons are assigned to the wrong channel as can be seen from figure D.6. In summary, in both evaluation experiments the 


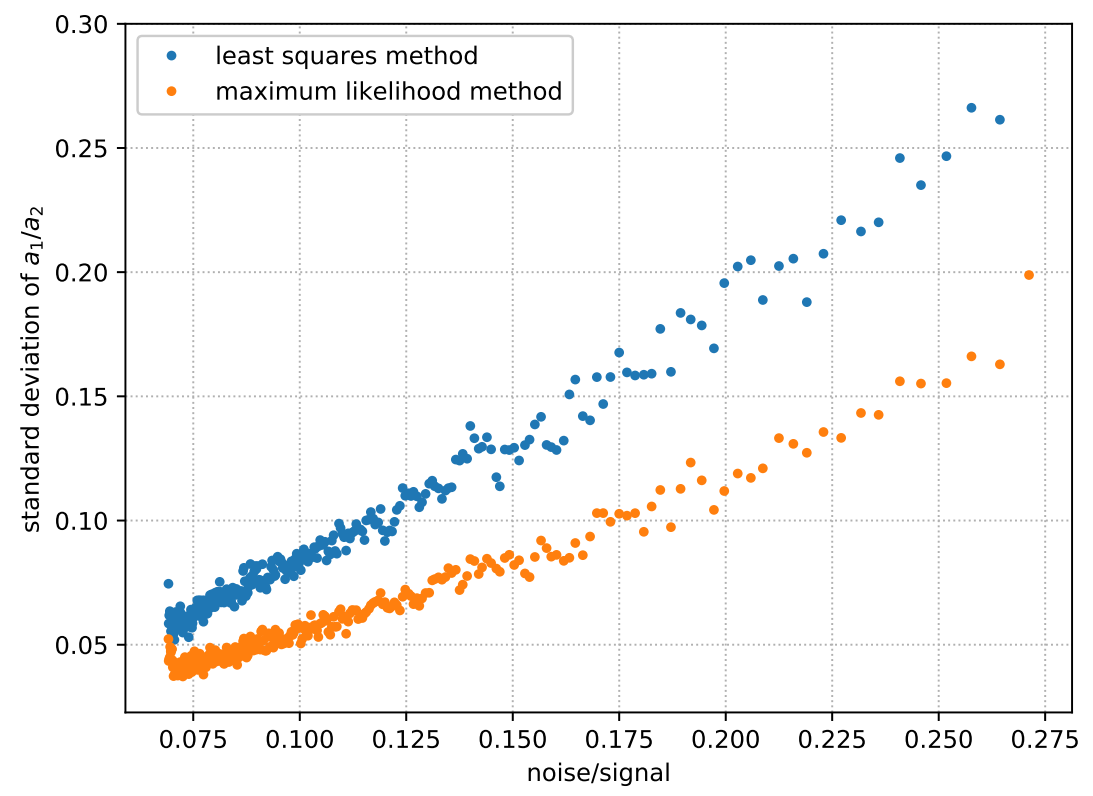

FIGURE 3.22: Evaluation of decomposition algorithms with simulated data. Decays were simulated by adding normalized reference histograms in a one-to-one ratio and adding Poisson noise. The simulated decays were decomposed and the noise-to-signal ratio was plotted against the standard deviation of the ratio $a_{1} / a_{2}$ of 100 decompositions.

maximum likelihood approach outperformed the least squares approach. This result was to be expected, since the maximum likelihood approach is based on the correct statistical model for the main source of noise in the data, namely shot or Poisson noise. For this reason, the maximum likelihood method was used in the following to perform decomposition of FLIM and FLIM-STED images.

\section{Two-color FLIM imaging}

As mentioned earlier, for two-color confocal fluorescence lifetime imaging a Bxb1 landing pad cell line stably expressing a miRFP703-keratin fusion protein (P2432) was transfected with a construct for the transient expression of a V410-tomm20 fusion protein (P3279). Images were recorded using the commercial QUAD scanning microscope and the prototype SNSPD with imaging conditions described in table D.1. Subsequently, FLIM images were decomposed into two separate channels using the maximum likelihood method described above based on the recorded reference decays for the different fluorescent proteins. Figure 3.23 shows a representative FLIM image, the decomposition of this FLIM image into separate channels and the merged color-coded image.

For recording of FLIM-STED images, the same sample and microscope was used. The imaging conditions can be found in table D.1. In contrast to the confocal FLIM images, the decomposition could not be performed directly, since the fluorescence signal does not follow a simple exponential decay any more. Figure D.7 shows the accumulated fluorescence decay of a recorded FLIM-STED image. The decay is composed of a fast initial component and a slower second component. The fast component is composed of fluorescence photons from the center of the STED donut 


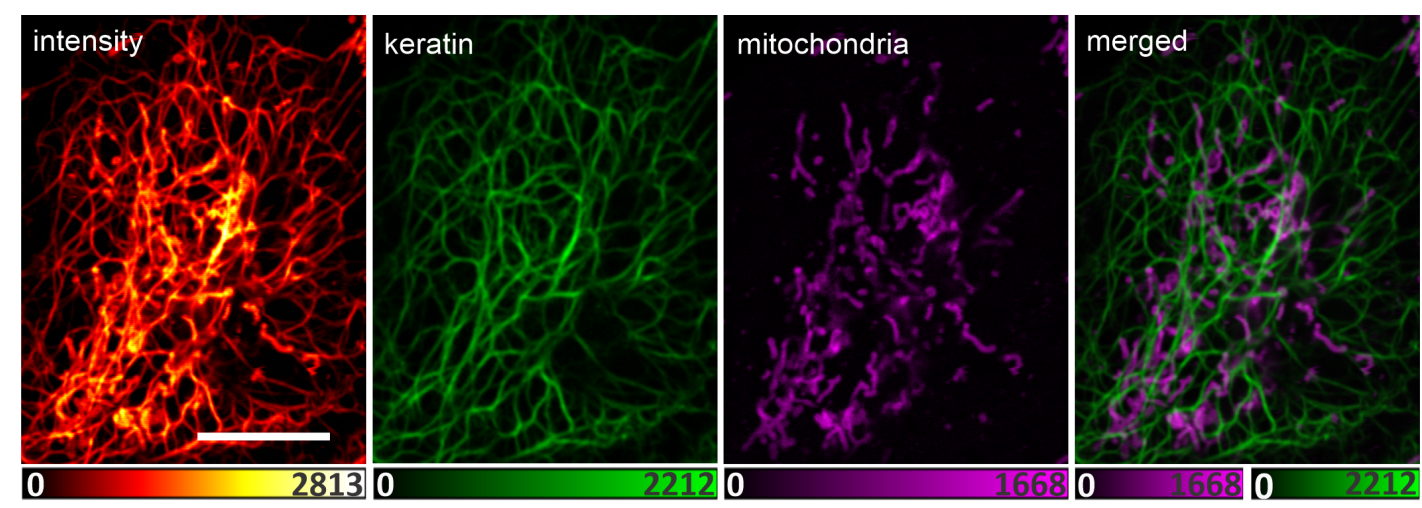

FIGURE 3.23: Two-color confocal fluorescence lifetime imaging of HeLa cells stably expressing miRFP703-keratin (P2432) and transiently expressing V410-tomm20 (P3279). From left to right: intensity image, decomposed keratin channel, decomposed tomm20 channel, merged two-color image. The decomposition was performed using the maximum likelihood method. Cells were incubated for $2 \mathrm{~h}$ in $25 \mu \mathrm{M}$ BV before imaging. Scale bar: $10 \mu \mathrm{m}$.
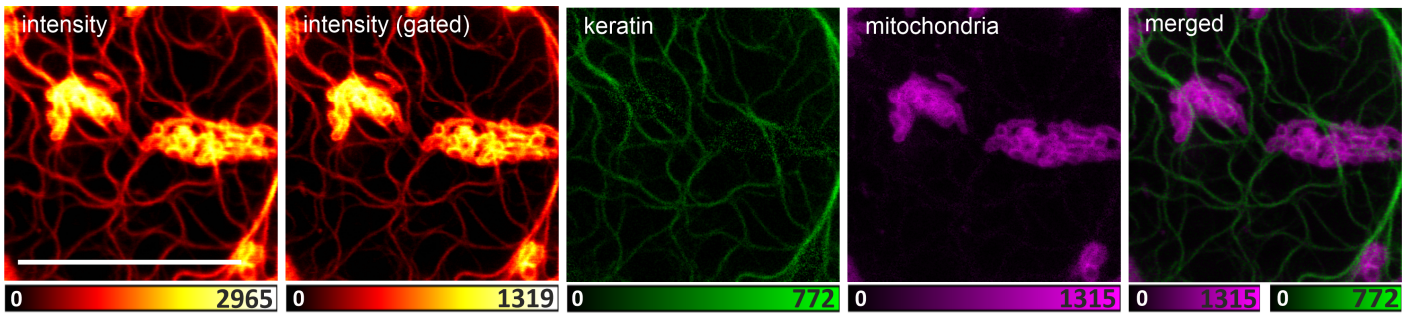

FIGURE 3.24: Two-color STED fluorescence lifetime imaging of HeLa cells stably expressing miRFP703-keratin (P2432) and transiently expressing V410-tomm20 (P3279). From left to right: intensity image, time gated intensity image, decomposed keratin channel, decomposed tomm 20 channel, merged two-color image. The decomposition was performed using the maximum likelihood method. Cells were incubated for $2 \mathrm{~h}$ in $25 \mu \mathrm{M}$ BV before imaging. Scale bar: $10 \mu \mathrm{m}$.

and from the periphery before the respective fluorophores could be deexcited by stimulated emission. The slow component represents the fluorescence decay of the fluorophores in the center. The fluorophores in the periphery are now deexcited by stimulated emission and do not contribute to the decay. For this reason, only the slow decay was used for decomposition of the FLIM images, while the other bins were discarded. This is similar to the practice of gated detection to increase the resolution in STED imaging by discarding the initial photons that are not affected by the STED laser [85]. A representative FLIM-STED image, the digitally gated FLIM STED image, the decomposed channels and the color-coded merged channels are shown in figure 3.24 .

In summary, by using the difference in fluorescence lifetime it was possible to perform two-color confocal and STED imaging of living mammalian cells expressing a miRFP703-keratin and a V410-tomm20 fusion protein. For the first time, two-color imaging was performed entirely in the NIR spectral region with an excitation beam at $670 \mathrm{~nm}$ and a depletion beam at $820 \mathrm{~nm}$. For this, the fluorescence lifetime images were decomposed into the respective color channels using custom software based 
on a Poisson noise model. This model takes into account the physical source of the majority of noise in the images, namely the probabilistic nature of photon emission. 


\section{Chapter 4}

\section{Discussion}

\subsection{Directed Evolution}

In this thesis, the fluorescence quantum yield of the NIR fluorescent protein miRFP703 was increased via directed evolution and fluorescence lifetime screening. Prior to this work, the approach was used for engineering of GFP-like fluorescent proteins. For example in 2010, Goedhart et al. identified bright cyan fluorescent protein variants by fluorescence lifetime screening [47]. Eight years later, in 2018, Manna et al. used a microfluidic sorter to increase the fluorescence quantum yield of the GFP-like fluorescent protein FusionRed by increasing its fluorescence lifetime [79].

\subsubsection{Screening and Selection}

The combined screening for fluorescence lifetime and fluorescence brightness in $E$. coli cells proved suitable for improving the fluorescence quantum yield of a bacterial phytochrome derived fluorescent protein. In the course of this work, the fluorescence lifetime of miRFP703 could be increased from 690 ps to 1120 ps for the final protein variant $\mathrm{V} 410$. With this, the fluorescence quantum yield was increased from $12 \%$ for miRFP703 to $21 \%$ for V410 while the brightness in the E. coli expression system increased 3-fold. This makes V410 the bacterial phytochrome derived fluorescent protein with the longest fluorescence lifetime and the highest quantum yield absorbing and emitting in the NIR spectral window beyond $650 \mathrm{~nm}$ to date. BV-binding fluorescent proteins with comparable fluorescence lifetimes and fluorescence quantum yields, e.g. smURFP with a fluorescence lifetime of $1400 \mathrm{ps}$ and a fluorescence quantum yield of $18 \%$ [109] and emIRFP670 with a fluorescence quantum yield of $14 \%$ [80], exhibit a blue-shift as compared to V410 and do not fall within the NIR spectral window. Since the fluorescence quantum yield is one of the major shortcomings of NIR fluorescent proteins engineered from bacterial phytochromes, the approach may prove an important tool for further developments in this regard.

For expression of the fluorescent protein variants, an E. coli based bacterial expression system was used. The choice of a bacterial expression system for screening allowed to perform many consecutive diversification and screening rounds within the timeframe of this thesis since plasmid based diversification and bacterial expression in E. coli is fast and efficient. The entire cycle consisting of PCR based diversification, screening and isolation of improved protein variants could be performed in three to four days.

However, one shortcoming of the current screening approach in E. coli was revealed by the comparison of the effective brightness in mammalian cells between 
V410 and miRFP703. The strongly increased fluorescence quantum yield, molecular brightness and brightness in E. coli cells of V410 compared to miRFP703, did not translate into effective brightness in mammalian cells under most conditions. Especially under low BV concentrations, V410 was, in the tested mammalian expression systems, not brighter than miRFP703. There are different possible explanations for this behaviour.

The BV affinity of V410 may have decreased as compared to miRFP703 during the screening process. The BV affinity was addressed indirectly in the screening approach by measuring the fluorescence intensity of E. coli colonies expressing the respective protein variant. However, the fluorescence intensity in E. coli colonies does not only depend on the BV affinity of the protein, but also on other factors, e.g., the colony size and the expression level. Those additional factors could not be addressed separately. It was assumed they would be stable and would not impair the measurement. If they were not stable, they may have compensated for decreased BV affinity so that the fluorescence intensity measured in E. coli colonies may not have reflected the BV affinity of the fluorescent protein variants.

Since there is no dedicated pathway for BV degradation in E. coli it is likely that the BV concentration in the E. coli expression system is much higher as the physiological BV concentration in mammalian cells [144, 24]. This may cause saturation of the fluorescent proteins in the E. coli expression system with BV. This again may lead to the effect that the fluorescence intensity in E. coli cells may not reflect the BV affinity.

Finally, reduced expression, impaired folding and maturation or increased degradation in mammalian cells may also explain the fact that the brightness of V410 in the E. coli expression system did not translate well to the mammalian expression system.

In all three possible explanations above, the core of the problem is a limited transferability of the results obtained in the E. coli expression system to the mammalian expression system. To avoid this problem entirely, screening in a mammalian expression system would be ideal. The easiest and most convenient mammalian expression system would be a transient expression from a transfected plasmid. The number of plasmids and, therefore, the resulting expression level of transiently transfected mammalian cells varies strongly and would not allow to draw conclusions on BV affinity based on fluorescence intensity of single cells. In principle, the same applies for transformation of bacterial cells. However, since an ensemble of E. coli cells forming a colony on an agar plate are measured simultaneously the differences in expression level between single cells are compensated.

A stable integration of the fluorescent protein variants into the genome would enable a homogeneous expression level in mammalian cells. The disadvantage of this approach would be that it is slower and less convenient than the expression in $E$. coli cells. For instance, most mammalian cell lines double approximately every $24 \mathrm{~h}$ while E. coli doubles approximately every $20 \mathrm{~min}$ [46]. As a result, less consecutive rounds of screening and diversification could be performed in a given timeframe. Additionally, the automated screening microscope would not be suitable for screening of mammalian cells. Instead a flow cytometry or microfluidic system would have to be used. 
A compromise between the transferability of a mammalian screening and the convenience of a bacterial screening could be a combined screening approach as described for example by Kamper et al. [65, 64]. In a combined screening approach, rounds of bacterial screening and rounds of mammalian screening could be alternated.

\subsubsection{Positions}

The final protein variant $\mathrm{V} 410$ had 4 mutations compared to the template miRFP703 [119]. In the following, the 4 mutations are discussed based on the crystal structure of miRFP703 and homologies with other bacterial phytochromes described in the literature.

\section{Position 193}

In V410, phenylalanine at position 193 was substituted by leucine. The amino acid is located in a flexible linker between $\beta$-sheets 7 and 8 in the GAF domain. In the crystal structure of miRFP703 the side chain of phenylalanine 193 is pointing away from the chromophore into the surrounding solution [119]. The substituted amino acid leucine, like phenylalanine, has a hydrophobic side chain but it is sterically less demanding. An effect on the chromophore via direct interactions is unlikely due to the relative distance and orientation of amino acid 193. However, the substitution of phenylalanine to leucine may effect the relative orientation and interaction of other amino acids with the BV chromophore.

\section{Position 202}

In V410 valin 202 was substituted by cystein. Position 202 and the neighbouring position 201 were described before to have an important role in chromophore stabilization $[4,118]$. Amino acid 202 is located in the GAF domain and is part of the highly conserved DIP motif. A kink in the polypeptide chain allows residues of amino acids in the DIP motif to engage in direct interactions with the BV chromophore [138]. It has been shown that mutating positions 201 and 202 does not only influence the fluorescence quantum yield by providing stabilization to the chromophore but may also alter the spectral properties of the protein. The spectrally distinct fluorescent proteins miRFP670, miRFP703 and miRFP709 have threonine and valine, leucine and valine and phenylalanine and isoleucine at positions 201 and 202 [119], respectively (see figure 4.1). At position 201, leucine and phenylalanine are unpolar residues, while threonine is a polar uncharged side chain. Valine and isoleucine at position 202 are both hydrophobic amino acid. Eventough more polar, cystein is regarded a hydrophibic amino acid like valine and isoleucine [8]. Two cysteine residues often form a disulfide bond with a length of approximately $2 \AA$ [27]. However, since no cysteine residue is located in an appropriate distance to cysteine 202 in V410 the formation of a disulfide bond seems unlikely here.

\section{Position 210}

Amino acid 210 is located in the chromophore binding pocket of the GAF domain in close proximity to the BV chromophore. The original tyrosine residue at position 210 forms a hydrogen bond with the carbonyl oxygens of BV's B ring [138] (compare to tyrosine 216 in figure 1.11). In V410, tyrosine 210 was replaced by histidine. Like tyrosine histidine is an aromatic amino acid. It can donate the nitrogen bound 


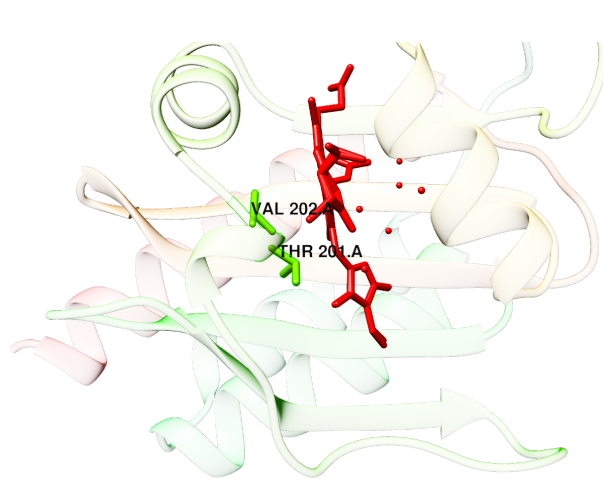

(A) miRFP670 (PDB ID: 5VIV)

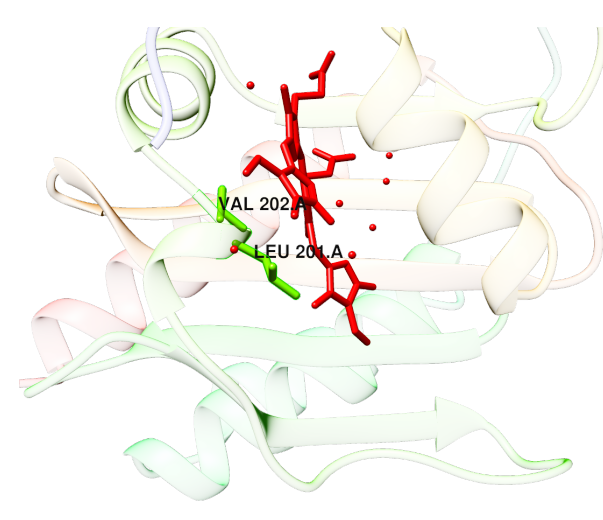

(B) miRFP703 (PDB ID: 5VIK)

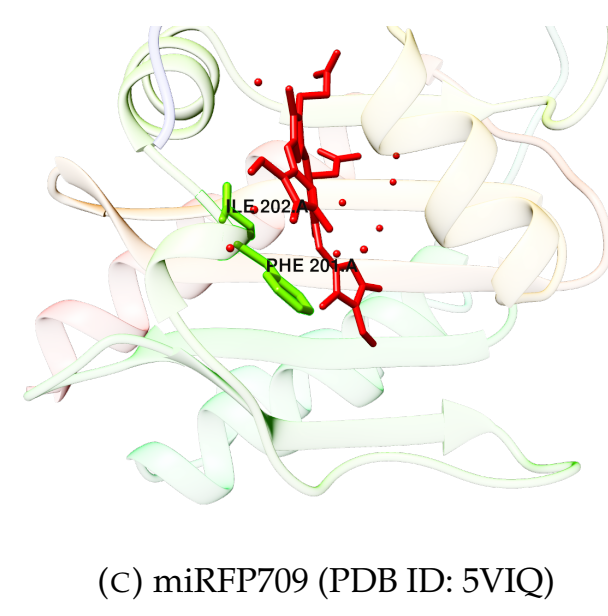

FIGURE 4.1: Positions 201 and 202 in spectrally distinct miRFP variants in (A) miRFP670, (B) miRFP703 and (C) miRFP709. The protein scaffold is shown in cartoon representation and rainbow color scheme (N-terminus, blue to C-terminus, red). The chromophore is colored in red. Positions 201 and 202 are shown in stick representation and are labeled. The figure is based on PDB structures 5VIV, 5VIK and 5VIQ [119].

hydrogen of its imidazole side chain to a hydrogen bond with the carbonyl oxygens of BV's B ring. Therefore, in principle histidine 210 in V410 may behave similar as tyrosine 210 in miRFP703.

\section{Position 253}

Mutation I253S was the key mutation for increasing the fluorescence lifetime and fluorescence quantum yield of miRFP703. Additionally, spectral changes in shape and position of absorption, excitation and emission bands of V410 were caused by the I253S mutation as can be seen from figure 3.6. In the closely related fluorescent protein miRFP670 introducing a cysteine residue at position 253 provided an alternative BV binding site $[58,120,67]$. The differences in BV binding between miRFP703 and miRFP670 are shown in the respective crystal structures in figure 4.2. The binding of the chromophore via cystein 253 drastically increased the fluorescence quantum yield of miRFP670 as compared to miRFP703. Hontani et al. hypothesized that the effect can be explained by a more constrained thioether linkage between the protein and the chromophore in miRFP703 [58]. Additionally, they showed that the 
$\pi$-electron conjugation to BV's A-ring is broken in miRFP670 causing the blue-shift. In the case of V410, mutation I253S does not allow for an alternative thioether linkage to BV. An ether linkage to the chromophore via position 253 seems unlikely due to the reduced reactivity of serine's alcohol group compared to cystein's thiol group. However, it is likely that the substitution of the unpolar amino acid isoleucine by the polar amino acid serine enables strong non-covalent interactions between the BV chromophore and the amino acid at position 253.

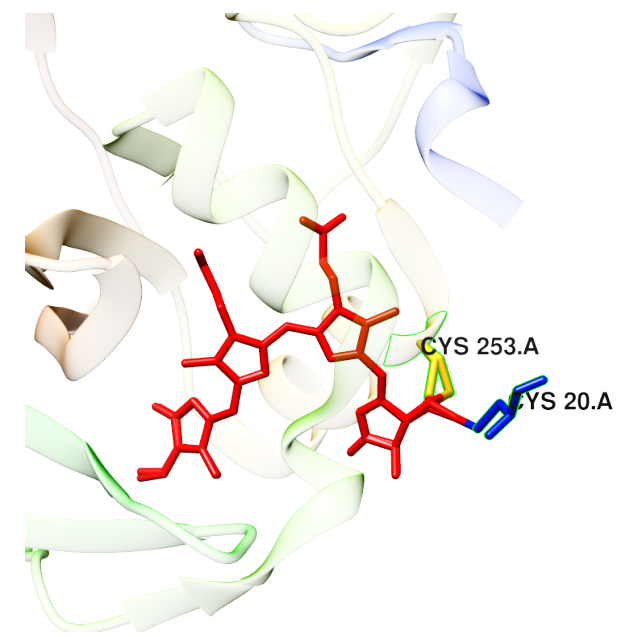

(A) miRFP670 (PDB ID: 5VIV)

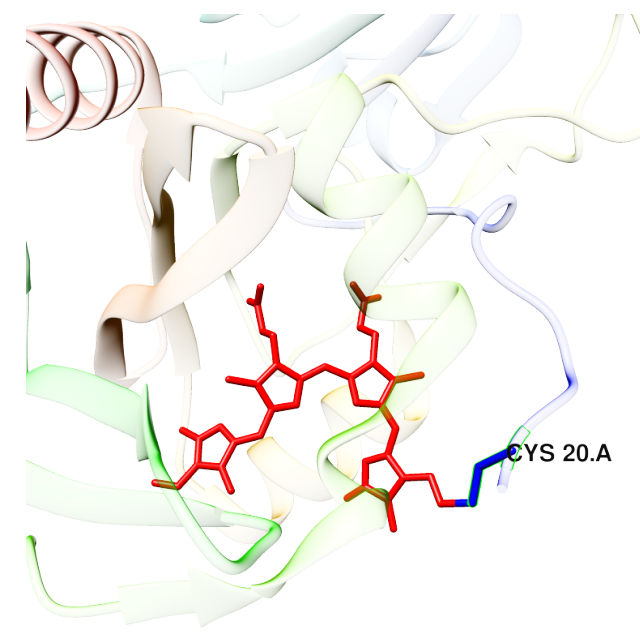

(B) miRFP703 (PDB ID: 5VIK)

FIGURE 4.2: BV binding in miRFP variants. (A) BV binding of miRFP670 via C20 and C253. (B) BV binding of miRFP703 via C20. The protein scaffold is shown in cartoon representation and rainbow color scheme (N-terminus, blue to C-terminus, red). The chromophore is colored in red. Amino acids involved in BV binding are labeled and shown in stick representation. The figure is based on PDB structures 5VIV and 5VIK [119].

\subsection{Characterization}

\section{Fluorescence Quantum Yield}

To measure the fluorescence quantum yield of bacterial phytochromes, two different approaches were used. The Quantaurus-QY system determines the absolute quantum yield by measuring the total amount of absorbed excitation photons and the total amount of emitted fluorescence photons. The fluorescence quantum yield can then be calculated as the ratio of those values. Due to the nature of the measurement, all absorbing molecules in the sample will contribute to the amount of absorbed photons while only fluorescent molecules can contribute to the total amount of emitted photons. In this sense, the measurement actually determines the quantum yield of the entire solution including, e.g., non-fluorescent protein species, free chromophore and protein of interest.

The second method to determine the fluorescence quantum yield used in this work is based on the modification of radiative transitions of emitters in a nanocavity and was introduced by Chizhik et al. in 2013 [22]. For this method, only fluorescent molecules contribute to the measured quantum yield. Non-fluorescent molecules 
are ignored by the measurement.

Since different values were obtained from both methods the conclusion must be drawn, that there were additional molecules in the purified protein solutions that did absorb NIR excitation light but did not show fluorescence. Different molecules are eligible. Free BV chromophore would absorb NIR excitation light but would not emit fluorescence since deexcitation is achieved via non-radiative pathways. However, the amount of free BV chromophore in the purified protein solutions should be low due to several buffer exchanges in the course of protein purification. Another possible explanation for the observed differences in fluorescence quantum yield is the presence of additional non-fluorescent bacterial phytochrome species in the purified protein solution. This non-fluorescent species could be made up of partially degraded, incompletely folded or misfolded protein or a mix of all of the former. Either way, the ratio of fluorescent and non-fluorescent but NIR-absorbing molecules is likely to vary between different purifications. Consequently, the values measured for the fluorescence quantum yield using the Quantaurus-QY system will vary accordingly. Therefore, the values measured with the second method seem to be more reliable and better suited for comparison between different bacterial phytochrome based NIR fluorescent proteins.

\subsubsection{Spectral Properties}

As described before in section 3.3, the shape of the fluorescence emission peak for V410 was found to be concentration depended. At low protein concentrations the emission spectrum of V410 exhibits a maximum around $700 \mathrm{~nm}$ with a pronounced shoulder at $670 \mathrm{~nm}$ as seen in figure 3.6c. With increasing the protein concentration the shoulder diminished until it disappeared entirely as shown in figure 3.7b.

A variety of different effects have been reported that can cause spectral shifts in protein spectra. It has been shown that aggregation and unfolding can cause spectral changes in the fluorescence emission spectra of proteins [34, 29, 74, 33]. For example, the ratio between GFP's absorption peaks at $395 \mathrm{~nm}$ and $470 \mathrm{~nm}$ is highly variable depending upon temperature, ionic strength, protein concentration and $\mathrm{pH}$ [141].

Naturally, phytochromes assemble into homodimers [149, 6]. A crystal structure of $R$. palustris bacterial phytochrome from Bellini et al. indicates that PAS and GAF domains are not involved in the dimerization interface (PDB ID: 4EHO [6]). However, in P. aeruginosa bacterial phytochrome the helical bundles of the PAS, GAF and PHY domain contribute to the dimer interface as demonstrated by Yang et al. (PDB ID: 3C2W [149]). For the engineered BV binding fluorescent protein SMURFP the chromophore is directly involved in homodimer formation as discussed by Rodriguez et al. [109]. At a protein concentration of $10 \mu \mathrm{M}$, no dimerization tendency was observed for V410 in SEC measurements (see figure 3.8a). However, with increasing protein concentration dimerization and oligomerization tendencies increase and at some point homodimer formation may occur. Dimerization of V410 may alter the spectral properties of the protein causing the spectral differences observed in the emission spectra of V410 recorded at different concentrations. Especially, when the dimerization interface involves the BV chromophore or the direct chromophore vicinity as observed for SMURFP [109] spectral changes in the excitation and emission spectra must be expected. Even if the local chromophore vicinity 
is not directly involved in dimerization, spectral changes may occur upon dimerization due to global restructuring of the protein scaffolds. In this context, it is interesting to note that the shoulder at $670 \mathrm{~nm}$ measured for V410 at low protein concentrations approximately coincided with the main emission peak of miRFP670 [119] as can be seen in figure 4.3.

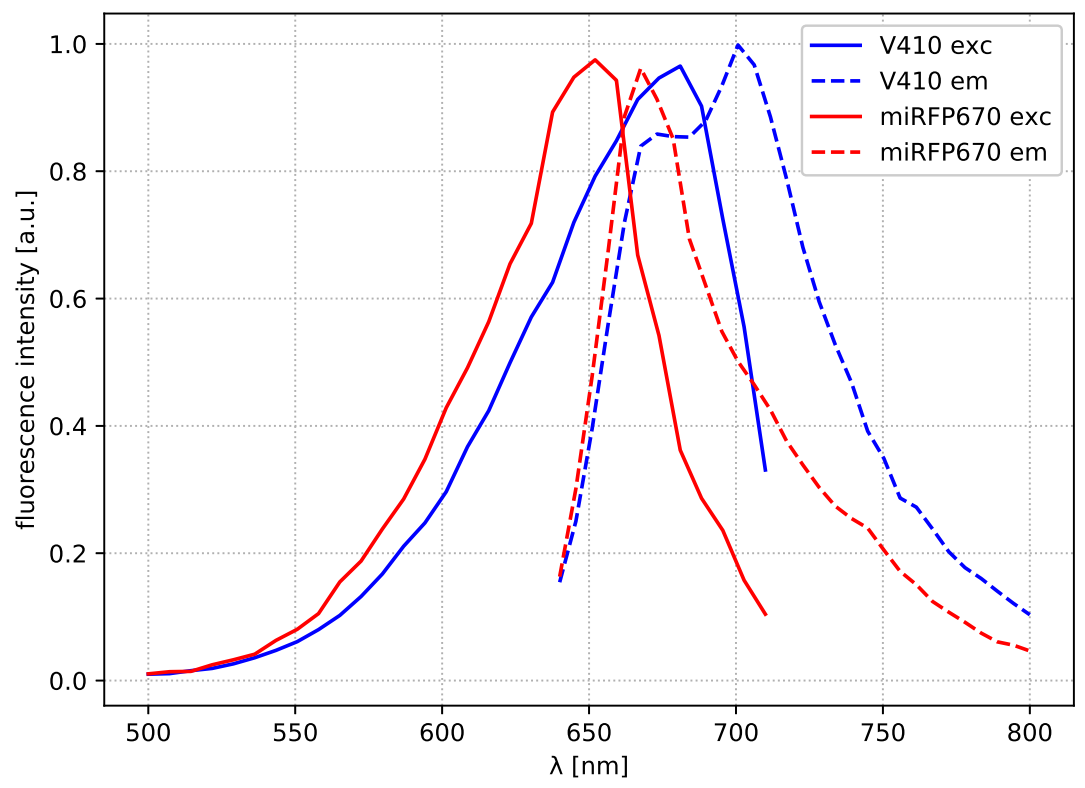

FIGURE 4.3: Comparison between excitation and emission spectra of protein variant V410 and miRFP670 [119].

\subsection{Imaging}

Prior to this thesis, several articles have been published describing the use of bacterial phytochrome based NIR fluorescent proteins for live-cell fluorescence imaging. In 2009, Shu et al. demonstrated NIR fluorescence imaging of engineered fluorescent protein IFP1.4 in living mice mediated by adenovirus serotype 5 [123]. Later an improved variant of IFP1.4, namely IFP2.0, was used for neuronal imaging of Drosophila melanogaster larvae in 2014 by Yu et al. [152]. Publications involving NIR fluorescence imaging of mammalian cells are, e.g., Shcherbakova et al. [119], Oliinyk et al. [90], Kamper et al. [65] and Matlashov et al. [80]. Of particular interest for this thesis are the publications of Kamper et al. from 2018 [65] and Matlashov et al. from 2020 [80], as they demonstrated STED imaging with bacterial phytochrome based NIR fluorescent proteins. As mentioned earlier, Kamper et al. employed the protein SNIFP derived from the engineered protein WiPhy [4], while Matlashov et al. used improved variants of the miRFP family [119]. Both Kamper et al. and Matlashov et al., used transiently transfected mammalian cells for fluorescence imaging.

In this thesis, fluorescence imaging was demonstrated using transiently transfected mammalian cells, Bxb1 landing pad cell lines and CRISPR/Cas-9 genome edited mammalian cell lines. While transiently transfected mammalian cells are an established model system for fluorescence imaging of bacterial phytochrome based NIR fluorescent proteins [119, 90, 65, 80], Bxb1 landing pad cell lines and stable CRISPR/Cas-9 genome edited cell lines have not been used in this context before. 


\subsubsection{Confocal Imaging}

Confocal imaging of different cellular localisations of V410 demonstrated the broad applicability of the protein as a fusion tag for fluorescence microscopy.

It was possible to label highly dynamic cellular structures in living mammalian cells like the microtubule cytoskeleton. The dynamics of the mictrotubule cytoskeleton could be followed over the course of several hours with only moderate photo bleaching and no signs of phototoxic effects caused by elongated exposure of the living sample with NIR excitation light. Also a fusion protein consisting of V410 and $\mathrm{H} 2 \mathrm{~B}$ was correctly localised in the nucleus. Again, no signs of toxic effects were found in the confocal images.

In order to further evaluate and quantify the phototoxicity of NIR fluorescence imaging and to investigate the phototoxicity of NIR imaging compared to imaging in the visible region, more sensitive experiments must be performed in the future. Goryaynov et al. measured the release of different indicators for cellular stress to evaluate the immediate cellular response to phototoxic stress caused by fluorescence imaging [49]. The release of $\mathrm{Ca}^{2+}$ was quantified via the $\mathrm{Ca}^{2+}$-sensor Fluoforte, mitochondria depolarization was measured through the decrease in fluorescence of JC-1 and reactive oxygen species were monitored using MitoSOX, CMFDA and Enzo Total ROS/Superoxide indicator. Another measure for cell toxicity was used by Pennacchietti et al. [93]. They used the increase in DNA repair puncta visualized with a fusion construct of XRCC1 with the fluorescent protein EYGFP to quantify phototoxic effects of fluorescence imaging on DNA integrity. Long time phototoxic effects can be quantified for example by observing the mortality rate of living cells after fluorescence imaging.

\section{Confocal Movies}

The photostability of V410 in confocal imaging was demonstrated by recording 1000 consecutive confocal frames of entire HeLa cells transiently expressing a Map2-V410 fusion construct. After 1000 consecutive frames with an excitation light dose of $1.1 \mathrm{~kJ} \mathrm{~cm}^{-2}$ the normalized frame intensity reached approximately $60 \%$ of the initial frame intensity.

Matlashov et al. demonstrated a similar bleaching behaviour for confocal recordings of fluorescent protein emiRFP703 [80]. However, in contrast to the measurements of Matlashov et al., where they recorded only a small region of the cell, in this work the entire cell was recorded. This approach measures the true confocal bleaching behaviour of the fluorescent protein more accurately, since less bleached protein can be replaced by unbleached protein from outside the imaging region. In summary, V410 and emiRFP703 performed comparably well in consecutive confocal imaging concerning photo bleaching. However, V410 showed this performance under more demanding imaging conditions since in contrast to emiRFP703 the entire cell was imaged over the course of several hours. 


\subsubsection{STED Imaging}

\section{Implementation}

To find the ideal depletion wavelength for STED imaging, the correlation between the STED wavelength and photo bleaching, reexcitation and the observed STED efficiency was investigated. The effect of reexcitation decreased for increasing STED wavelength between 790 to $840 \mathrm{~nm}$ following the tail of the excitation spectrum of V410. This is in agreement with the literature, for example with results by Kamper et al. [65] obtained with the NIR fluorescent protein SNIFP. The effect can be explained physically since the amount of reexcitation from the STED beam is correlated to the spectral overlap between the STED spectrum and the excitation spectrum of the protein. The bleaching halftime increased for increasing STED wavelength between 790 and $840 \mathrm{~nm}$. This is likely due to increased cycling of the fluorescent molecule between the states $S_{0}$ and $S_{1}$ for shorter STED wavelength. For example, this effect was described by Oracz et al. in 2017 [91].

The data recorded for the correlation between STED wavelength and STED efficiency and STED offset exhibited a typical behaviour. The saturation intensity is typically dominated by the stimulated emission cross section. The larger the overlap between the STED spectrum and the emission spectrum of the fluorophore the larger the stimulated emission cross section and the smaller the saturation intensity. This wavelength dependency was experimentally demonstrated for example by Vicidomini et al. in 2012 [137] for the fluorescent dye Atto-647N or in 2016 by Bordenave et al. [15] for the fluorescent stain NileRed. Those experiments are in agreement with the measurements of the STED efficiency performed in this work. The only exception being the value obtained for $P_{\text {sat }}$ at $830 \mathrm{~nm}$ as it is higher than the value at $840 \mathrm{~nm}$. The STED offset was maximal at $790 \mathrm{~nm}$ and monotonically decreased for longer wavelengths. This behaviour suggests that the offset is dominated by reexcitation of molecules via the STED beam. A STED background due to reexcitaion via the STED beam was described for example by Rittweger et al. [107] or Gao et al. [44, 45].

\section{STED Imaging}

As demonstrated in figure 3.16, it was possible to perform STED imaging in the NIR spectral region using an endogenously tagged CRISPR cell line expressing a fusion construct of vimentin with V410. Filaments of vimentin have a diameter of approximately $10 \mathrm{~nm}[28,56,128,125,88,55]$ and the CBD of bacterial phytochromes has a diameter of approximately $6 \mathrm{~nm}$. Therefore, the diameter of vimentin filaments endogenously labeled with V410 is in the order of $25 \mathrm{~nm}$. This reflects the best resolution achievable under ideal imaging conditions. In the actual experiment perturbations caused by motion artefacts and aberrations lower the achievable resolution. For example Wurm et al. identified a resolution of $25 \mathrm{~nm}$ in STED microscopy of vimentin filaments labeled with Abberior STAR635P for an idividual antibody cluster [145] and Butkevich et al. measured a resolution of approximately $50 \mathrm{~nm}$ in STED microscopy of vimentin-HaloTag filaments after incubation with the fluorescent dye 610CP [20]. In this work, resolutions from around $80 \mathrm{~nm}$ down to $40 \mathrm{~nm}$ were measured for line profiles of STED images recorded with living genome edited U2OS cells expressing the fusion construct V410-vimentin. The achieved resolutions are substantially better than the resolutions achieved previously with NIR fluorescent protein SNIFP $[64,65]$ and comparable with the resolution achieved with emiRFP 
variants [80].

In STED imaging of emiRFP variants, an excitation wavelength of $640 \mathrm{~nm}$, outside the NIR spectral window from 650 to $900 \mathrm{~nm}$, was used [80]. For this work, an excitation beam at $660 \mathrm{~nm}$ was used to truly benefit from the low absorbance of water, haemoglobin and oxyhaemoglobin in this spectral region beyond $650 \mathrm{~nm}$. Additionally, compared to Matlashov et al. [80] the STED wavelength employed in this thesis was red-shifted by $45 \mathrm{~nm}$ to $820 \mathrm{~nm}$. Due to the red-shift, phototoxicity induced by the STED beam is potentially reduced [49].

Many efforts have been made to enable STED imaging of living cells with minimal perturbation of the sample. In this regard, Kamper et al. took an important step by recording the first STED images in the NIR region of mammalian cells expressing SNIFP fusion constructs. However, due to the limited brightness of SNIFP transiently transfected cells were used with non-physiological expression levels of the fusion construct and $25 \mu \mathrm{M}$ BV had to be added before imaging. STED imaging of emiRFP703 could be performed without the addition of BV, but Matlashov et al. still used transiently transfected cells. In this work, STED imaging of mammalian cells stably expressing a V410-keratin fusion protein from a Bxb1 landing pad was performed without the addition of BV beforehand. With this, a further improvement towards physiologically relevant NIR STED imaging was realized.

\section{STED Movies}

STED movies of transiently transfected HeLa cells expressing Map2 tagged with V410 were recorded. After 100 consecutive STED frames with a STED dose of approximately $3.8 \mathrm{~kJ} \mathrm{~cm}^{-2}$ the normalized integrated intensity dropped to approximately $50 \%$ of the initial normalized integrated intensity. A direct comparison between V410 and the NIR fluorescent proteins emiRFP703 and SNIFP is difficult since different cellular structures and different imaging parameters were used. SNIFP reached $50 \%$ of the initial frame intensity after approximately eight frames with a STED dose of $244 \mathrm{~kJ} \mathrm{~cm}^{-2}$ and a STED wavelength of $860 \mathrm{~nm}$, while emiRFP703 reached $50 \%$ of the initial frame intensity after 21 frames with a STED dose of $6.7 \mathrm{~kJ} \mathrm{~cm}^{-2}$ and a STED wavelength of $775 \mathrm{~nm}$ [80].

\subsubsection{FLIM Imaging}

One benefit in fluorescence imaging is the superior contrast since only fluorescently labeled structures are visible. However, this property makes it difficult to put the labeled structure into a cellular context. With multi-color imaging of biological samples the contextualisation of labeled structures becomes possible. For this, different cellular structures are typically labeled with spectrally different fluorescent molecules. However, this approach is in general not compatible with imaging entirely in the NIR spectral region. In this work, the spectrally similar NIR fluorescent proteins V410 and miRFP703 were used for two-color fluorescence imaging. The structures labeled with the different proteins were separated based on their respective fluorescence lifetime and not based on their spectral characteristics. For the first time, this approach enabled two-color confocal and STED imaging entirely in the NIR spectral region without the need of an additional excitation and STED beam outside the NIR spectral window. The approach is in principle expandable to more than two different NIR fluorescent proteins. 


\section{Decomposition Algorithms}

The evaluation of the implemented decomposition algorithms showed that the maximum likelihood approach outperformed the least squares approach for all scenarios. This was not surprising as the maximum likelihood approach assumed the correct noise model for fluorescence lifetime data. The Poisson distribution models the probability distribution of events happening at a constant rate independently from each other in a defined time interval. The process of spontaneous emission of a photon is such a process. By finding the most likely distribution to fit the decay data using Poisson statistics, errors in bins with high photon counts are automatically penalized more than errors in bins with low photon counts. Using the least squares approach for fitting the decay data, errors in all bins are treated equally, hence errors in bins with high photon counts are underestimated and errors in bins with low photon counts are overestimated.

However, despite the inferior precision of the least squares decomposition algorithm as compared to the maximum likelihood algorithm it may be preferable in situations where large amounts of FLIM data need to be decomposed fast. As the maximum likelihood algorithm was implemented in this work, the cost function needs to be minimized separately for every pixel in the FLIM image. In contrast, for the least squares approach only matrix equation (3.9) had to be solved.

In summary, in the scope of this work a screening system was planned and built to screen for bacterial phytochrome variants with increased fluorescence lifetime and fluorescence quantum yield. With the help of this screening system the NIR fluorescent protein V410 could be generated from miRFP703. It exhibited the longest fluorescence lifetime and the highest fluorescence quantum yield of all engineered NIR fluorescent proteins absorbing and emitting in the NIR spectral window between 650 to $900 \mathrm{~nm}$ to date. Additionally, it was demonstrated that the protein performed well in NIR confocal and STED imaging of various cellular structures in mammalian cells including endogenously labeled mammalian cells. For the first time, two-color all NIR confocal and STED FLIM imaging was performed within this work.

\subsubsection{Outlook}

\section{Directed Evolution and Screening}

As discussed above, a screening system based on the bacterial expression of the diversified protein variants does not address the parameters of chromophore affinity and expression, folding, maturation and degradation in mammalian cells. To address those issues, it is necessary that the current screening approach is complemented with screening rounds in a mammalian expression system.

To find further diversification strategies it may be helpful to obtain a crystal structure of the current protein variant V410. A crystal structure would help to understand the molecular basis of the increased fluorescence lifetime and fluorescence quantum yield of V410 and would enable to rationally improve those parameters further. The spectrally distinct set of fluorescent proteins miRFP670 (PDB ID: 5VIV), miRFP703 (PDB ID: 5VIK) and miRFP709 (PDB ID: 5VIQ) has been crystallized and the crystal structures have been solved with a resolution of around $1.3 \AA$ [119]. Since V410 is directly derived from miRFP703, it is likely that the crystallization conditions 
described for miRFP703 would also work for V410.

In order to more efficiently sample the sequence space for improved protein variants, elaborate computational methods could be employed in future efforts. For example, it is possible to model the high dimensional fitness landscape of fluorescent proteins using machine learning methods and with this knowledge enrich the pool of protein variants with more promising candidates as demonstrated by Saito et al. in 2018 [113].

\section{Imaging of Living Organisms}

The benefits resulting from imaging in the NIR spectral region are highly relevant for deep-tissue fluorescence imaging in living mammalian organisms. Only a small number of publications have been published demonstrating deep-tissue NIR fluorescence imaging. Shu et al. demonstrated adenovirus serotype 5 mediated NIR fluorescence imaging in mice [123]. Neuronal imaging of Drosophila melanogaster larvae was performed by Yu et al. [152]. Shcherbakova et al. used the technique of hydrodynamic gene transfer [75] to express a $\mathrm{I} \kappa \mathrm{B} \alpha$-miRFP703 fusion construct in mouse liver [119].

The imaging experiments of V410 in living mammalian cells reported in this thesis were necessary steps towards live-cell NIR deep-tissue imaging. A simple model system to demonstrate the tissue penetration capabilities of NIR fluorescence imaging could be spheroids of mammalian cells. Together with Daniel Stumpf preliminary penetration experiments using spheroids of mammalian cells expressing V410 fusion constructs were performed (data not shown). Before proceeding to living mammalian organisms, V410 should be tested as fusion tag in more efficient model systems, e.g., Drosophila melanogaster, Caenorhabditis elegans or mammalian tissue culture. In this regard, first preliminary experiments in cooperation with Dr. Kamila Kiszka with V410 fused to the actin marker LifeAct [105] expressed in cultured brain slices from mouse embryos were successfully performed (data not shown). The results suggested the applicability of V410 in complex tissue samples. 


\section{Appendix A}

\section{Cloning Primer}

\begin{tabular}{c|l}
$\#$ & Sequence $\left(3^{\prime} \rightarrow{ }^{\prime}\right)$ \\
\hline \hline 1836 & TCCACCGGTCGCCACCATGGTGAGCAAGGGCGAGGAGCTGTTC \\
\hline 1837 & GTCGCGGCCGCTACTTGTACAGCTCGTCCATGCCGAGAG \\
\hline 4573 & CGTCTCGAGCTATGGATGATGATAT \\
\hline 4574 & CCGGTGGATCCTTACCTAGAAGCATTTGCGG \\
\hline 5570 & AGATCCGCTAGCATGGTGGGTCGGAACAGCGCC \\
\hline 5571 & TGGCGACCGGTGGATCCCCTTCCACATCATCTTCAGCC \\
\hline 7819 & GCTACAGATCTATGGTAGCAGGTCATGCC \\
\hline 7822 & TTCGAATTCGCTCTCAAGCGCGGTGATCCG \\
\hline 8348 & AACGAATTCGCTCTCAAGCGCGGTGATCCGC \\
\hline 8352 & TCGAAGCTTAGGCATGGTAGCAGGTCATGCCTCT \\
\hline 8357 & ATATTAGCGGCCGCCGGTAGTGGTTCAGGGGTAGCAGGTCATGC \\
\hline 8358 & CTCTGGC \\
\hline 8911 & CACTATTCCATGGTTAGCTCTCAAGCGCGGTGATCCG \\
\hline 8912 & TCCGCTAGCGCTACCGGTCGCCACCATGGTAGCAGGTCATGC \\
\hline 9561 & ATCCGCTAGCGCTAATGGTAGCAGGTCATGCC \\
\hline 9562 & CACTCGAGATCTGAGTCCGGAGCTCTCAAGCGCGGTGAT \\
\hline 9563 & CGACCGGTCGCCACCATGGTAGCAGGTCATGCC \\
\hline 9564 & AGCTGTGCGGCCGCTCAGCTCTCAAGCGCGGTGAT \\
\hline 9596 & ATCCGCTAGCGCTAATGGTAGCAGGTCATGCC \\
\hline 9595 & CTCGAGATCTGAGTCCGGAGCTCTCAAGCGCGGTGAT \\
\hline 9687 & GGATCCACCGGTCGCCACCATGGTAGCAGGTCATGCCTCT \\
\hline 9688 & TCTAGAGTCGCGGCCGCTAGCTCTCAAGCGCGGTGAT \\
\hline 9788 & CTTCGAATTCATGGTAGCAGGTCATGCC \\
\hline 9789 & GCGACCGGTTTCATGGTGGCAGGTCCAGGGTTCTCCTCCACGTC \\
& GCCAGCCTGCTTCAGCAGGCTGAAGTTAGTAGCTCCGCTTCCGC \\
TCTCAAGCGCGGTGAT
\end{tabular}

TABle A.1: Primers 



\section{Appendix B}

\section{Buffers and Media}

\begin{tabular}{|c|c|}
\hline Buffer & Composition \\
\hline Standard protein buffer & $100 \mathrm{mM}$ TRIS-HCl, $150 \mathrm{mM} \mathrm{NaCl}, \mathrm{pH} 7.5$ \\
\hline His binding buffer & $20 \mathrm{mM} \mathrm{Na}_{3} \mathrm{PO}_{4}, 500 \mathrm{mM} \mathrm{NaCl}, 20 \mathrm{mM}$ imidazole, $\mathrm{pH} 7.5$ \\
\hline His washing buffer & $20 \mathrm{mM} \mathrm{Na}_{3} \mathrm{PO}_{4}, 500 \mathrm{mM} \mathrm{NaCl}, 50 \mathrm{mM}$ imidazole, $\mathrm{pH} 7.5$ \\
\hline His elution buffer & $20 \mathrm{mM} \mathrm{Na}_{3} \mathrm{PO}_{4}, 500 \mathrm{mM} \mathrm{NaCl}, 500 \mathrm{mM}$ imidazole, $\mathrm{pH} 7.5$ \\
\hline 10x PCR buffer & $100 \mathrm{mM}$ TRIS-HCl, $500 \mathrm{mM} \mathrm{KCl}, 25 \mathrm{mM} \mathrm{MgCl}_{2}, \mathrm{pH} 8.3$ \\
\hline 10x Error prone buffer & $\begin{array}{l}100 \mathrm{mM} \text { TRIS-HCl, } 500 \mathrm{mM} \mathrm{KCl}, 70 \mathrm{MgCl}_{2}, 0.1 \%(\mathrm{w} / \mathrm{v}), \\
\text { pH } 8.3\end{array}$ \\
\hline 50x TAE buffer & $2 \mathrm{M}$ TRIS, $1 \mathrm{M} \mathrm{C}_{2} \mathrm{H}_{9} \mathrm{NaO}_{5}, 50 \mathrm{mM}$ EDTA, $\mathrm{pH} 7.2$ \\
\hline LB medium & $\begin{array}{l}11 \text { sterile water, } 5 \mathrm{~g} \mathrm{NaCl}, 5 \mathrm{~g} \text { yeast extract, } 10 \mathrm{~g} \text { peptone from } \\
\text { casein, } 5 \mathrm{ml} 1 \mathrm{M} \mathrm{NaOH} \text { to adjust } \mathrm{pH} \text {; autoclaved before use }\end{array}$ \\
\hline $\mathrm{pH}$ buffers & $\begin{array}{l}\text { pH 3-5.75: } 100 \mathrm{mM} \text { citric acid, } 150 \mathrm{mM} \mathrm{NaCl} \\
\text { pH 6-7: } 100 \mathrm{mM} \mathrm{KH}_{2} \mathrm{PO}_{4}, 150 \mathrm{mM} \mathrm{NaCl} \\
\text { pH 7.5-8.5: } 100 \mathrm{mM} \text { Tris, } 150 \mathrm{mM} \mathrm{NaCl} \\
\text { pH 9-9.5: } 100 \mathrm{mM} \text { glycine, } 150 \mathrm{mM} \mathrm{NaCl}\end{array}$ \\
\hline DMEM & $\begin{array}{l}4.5 \mathrm{~g} / \mathrm{l} \text { glucose, GlutaMAX }{ }^{\mathrm{TM}} \text {, phenol red, } 10 \%(\mathrm{v} / \mathrm{v}) \text { fetal } \\
\text { bovine serum, } 1 \mathrm{mM} \text { sodium pyruvate, } 100 \mathrm{U} / \mathrm{ml} \text { penicillin } \\
\text { and } 100 \mu \mathrm{g} / \mathrm{ml}\end{array}$ \\
\hline Freezing medium & DMEM supplemented with $20 \%$ FBS, $10 \%$ dimethyl sulfoxide \\
\hline
\end{tabular}

TABLE B.1: Buffers and Media 



\section{Appendix C}

\section{Python Code}

\section{C.1 Analysis of $\mathrm{pH}$ spectra}

LISTING C.1: Python code for fitting pH spectra

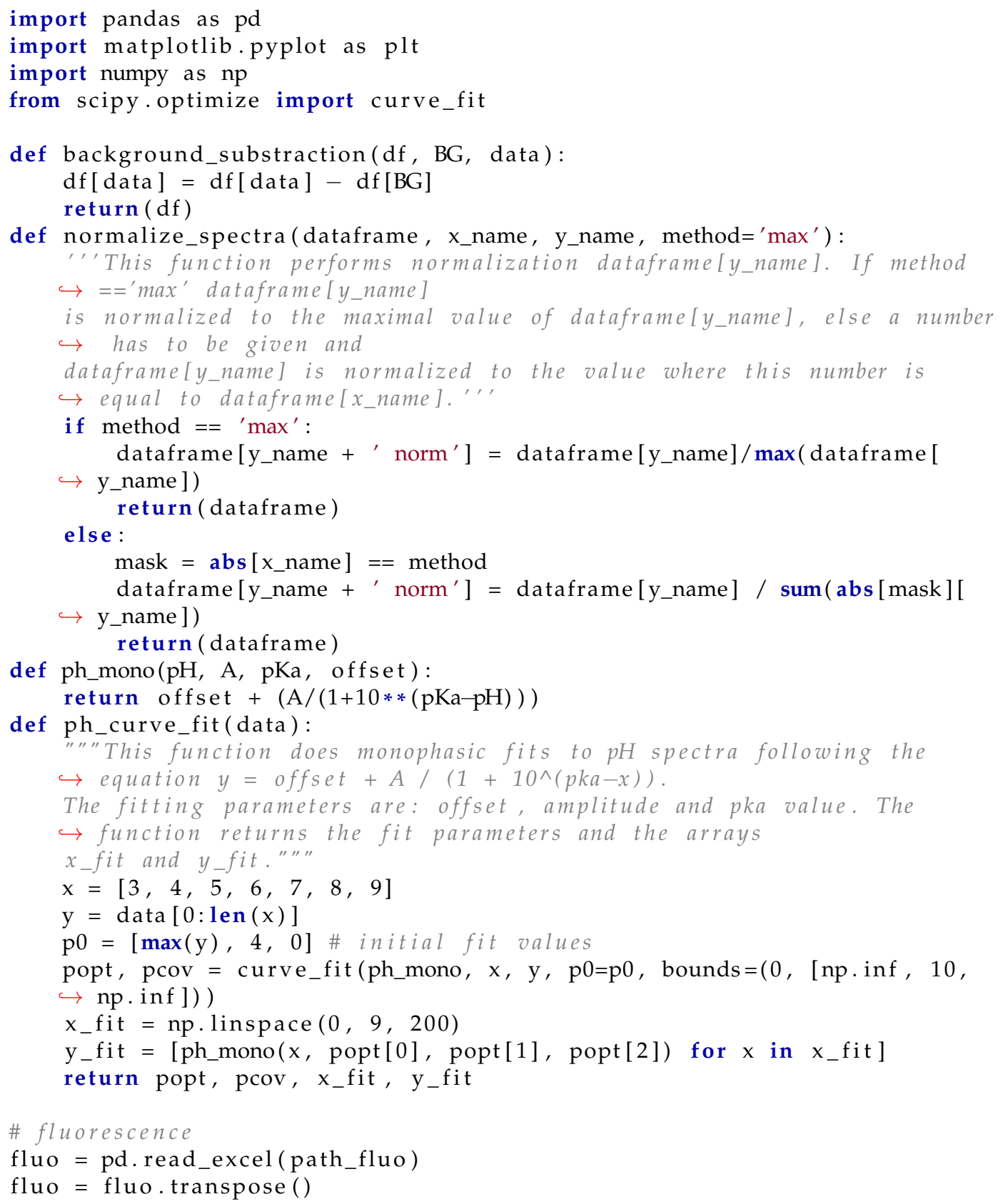




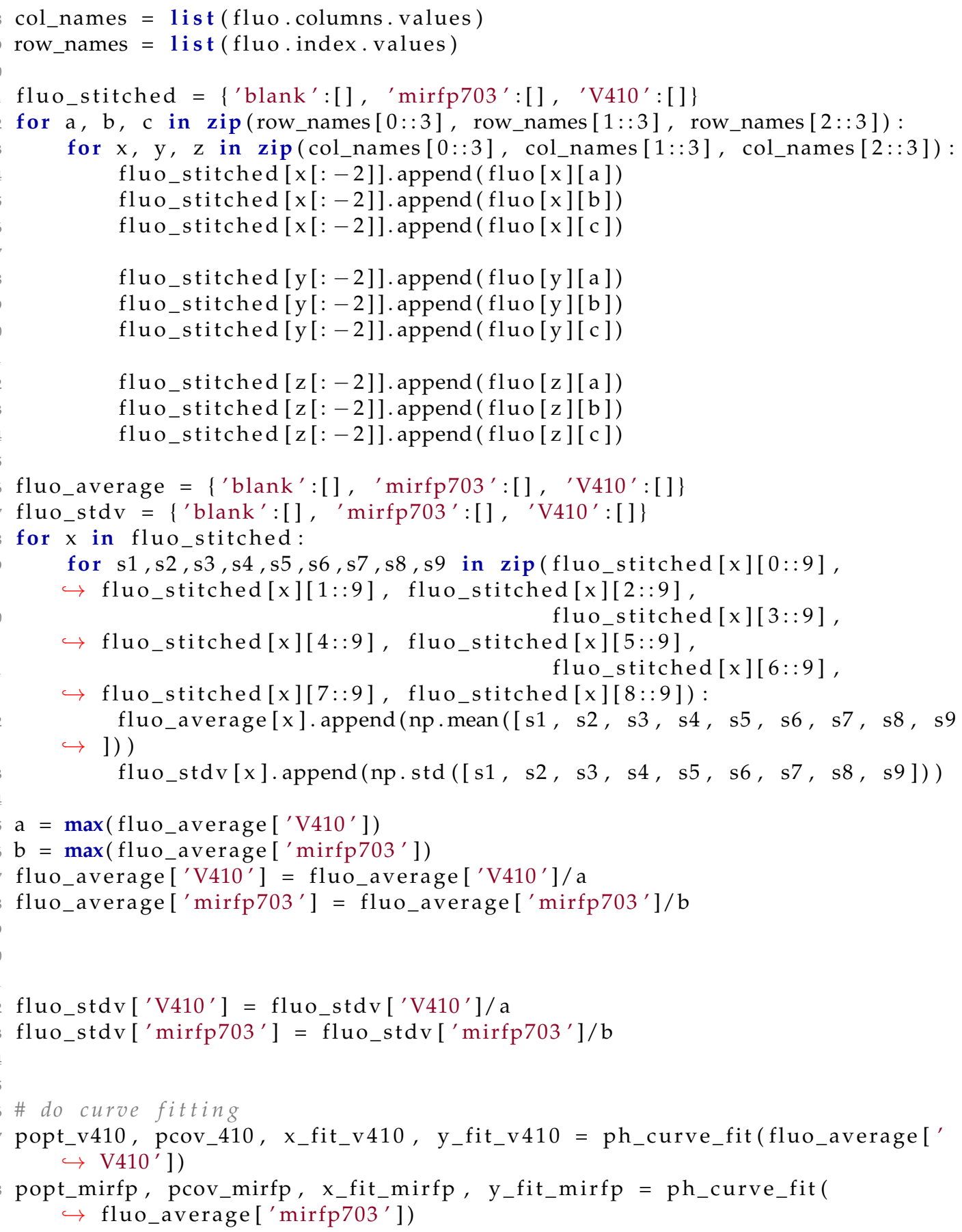

\section{C.2 Bleaching Analysis}

LISTING C.2: Python code for bleaching analysis

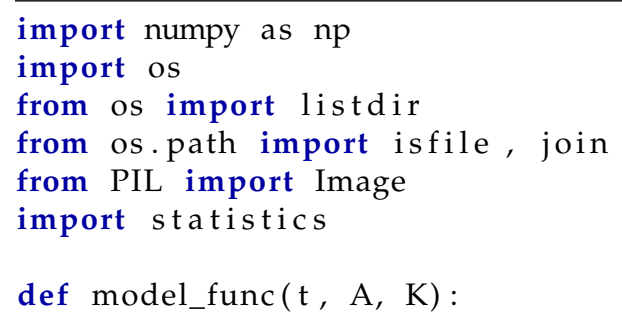




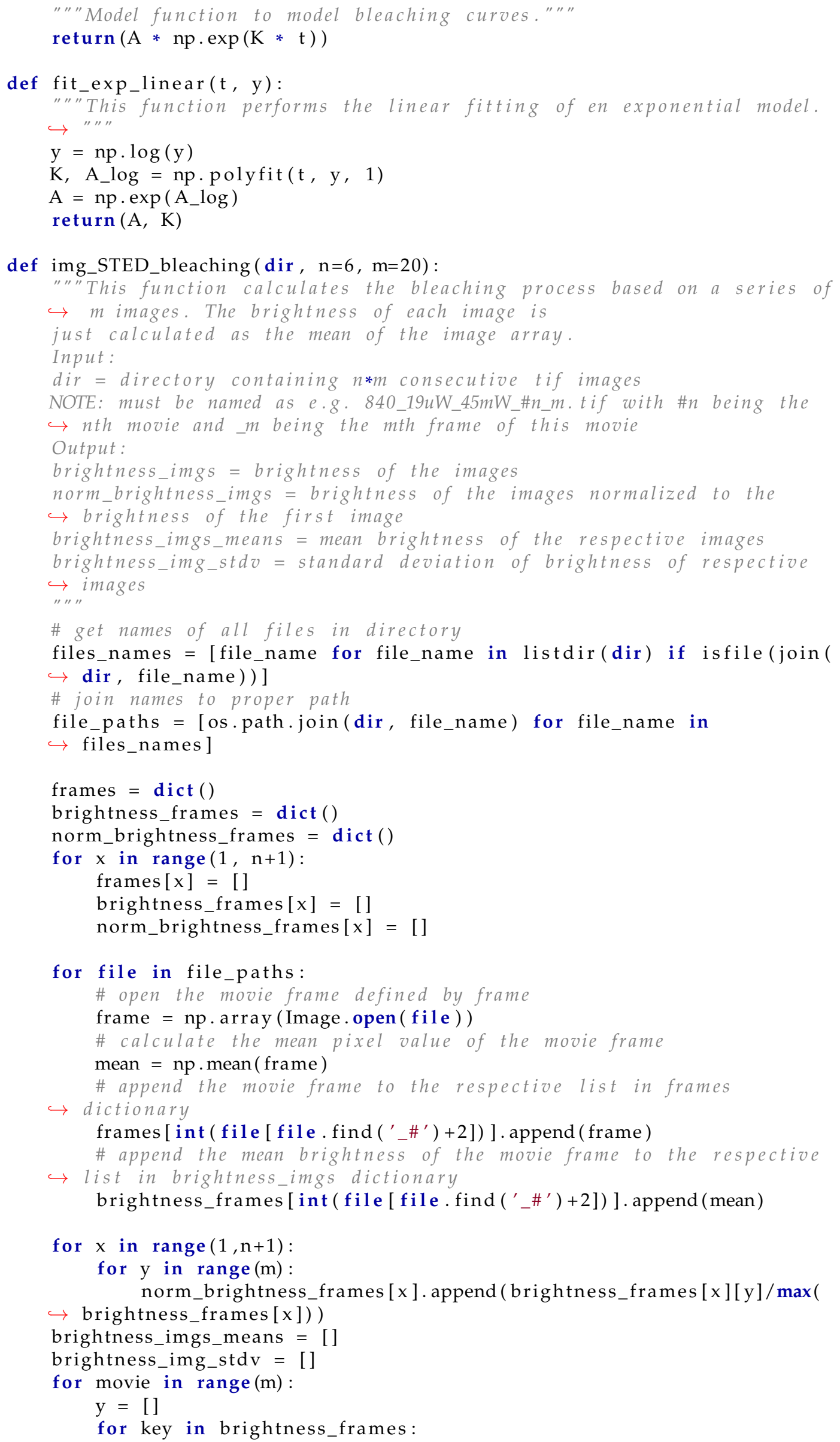




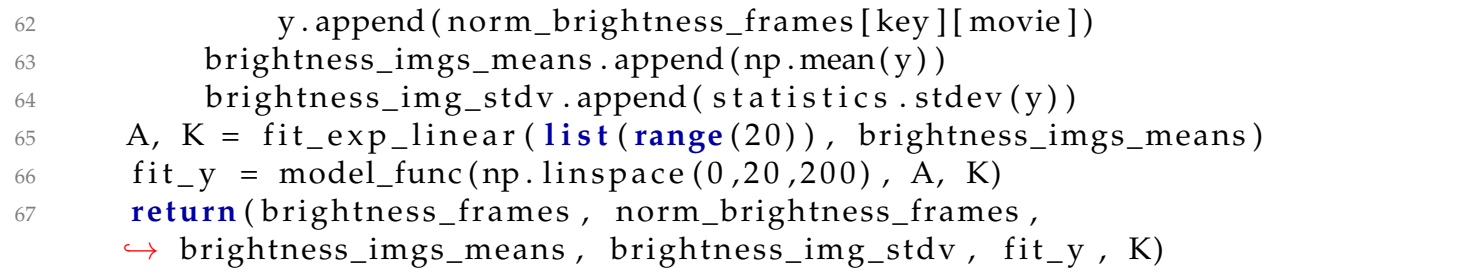

\section{C.3 Reexcitation}

LISTING C.3: Python code for reexcitation analysis

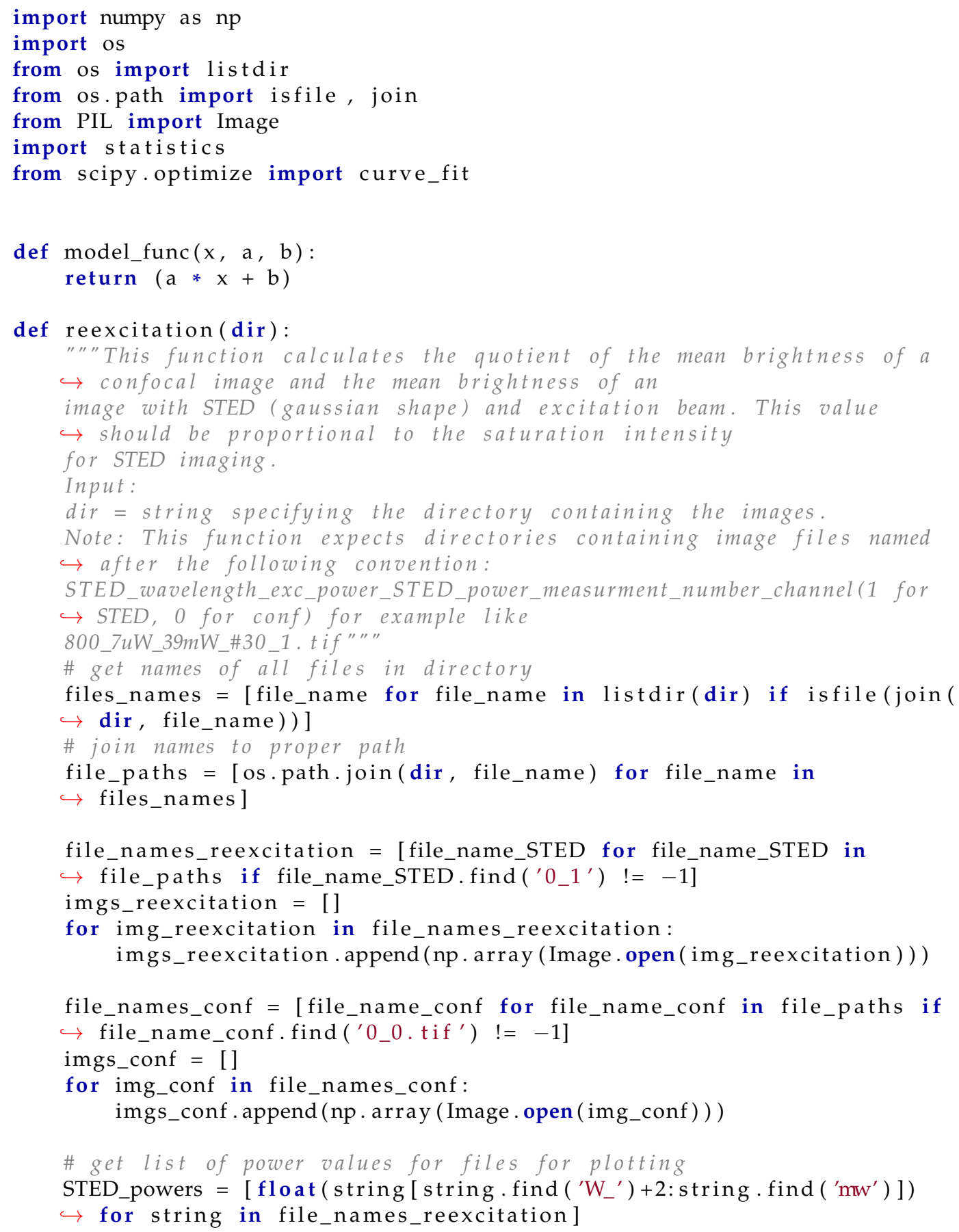




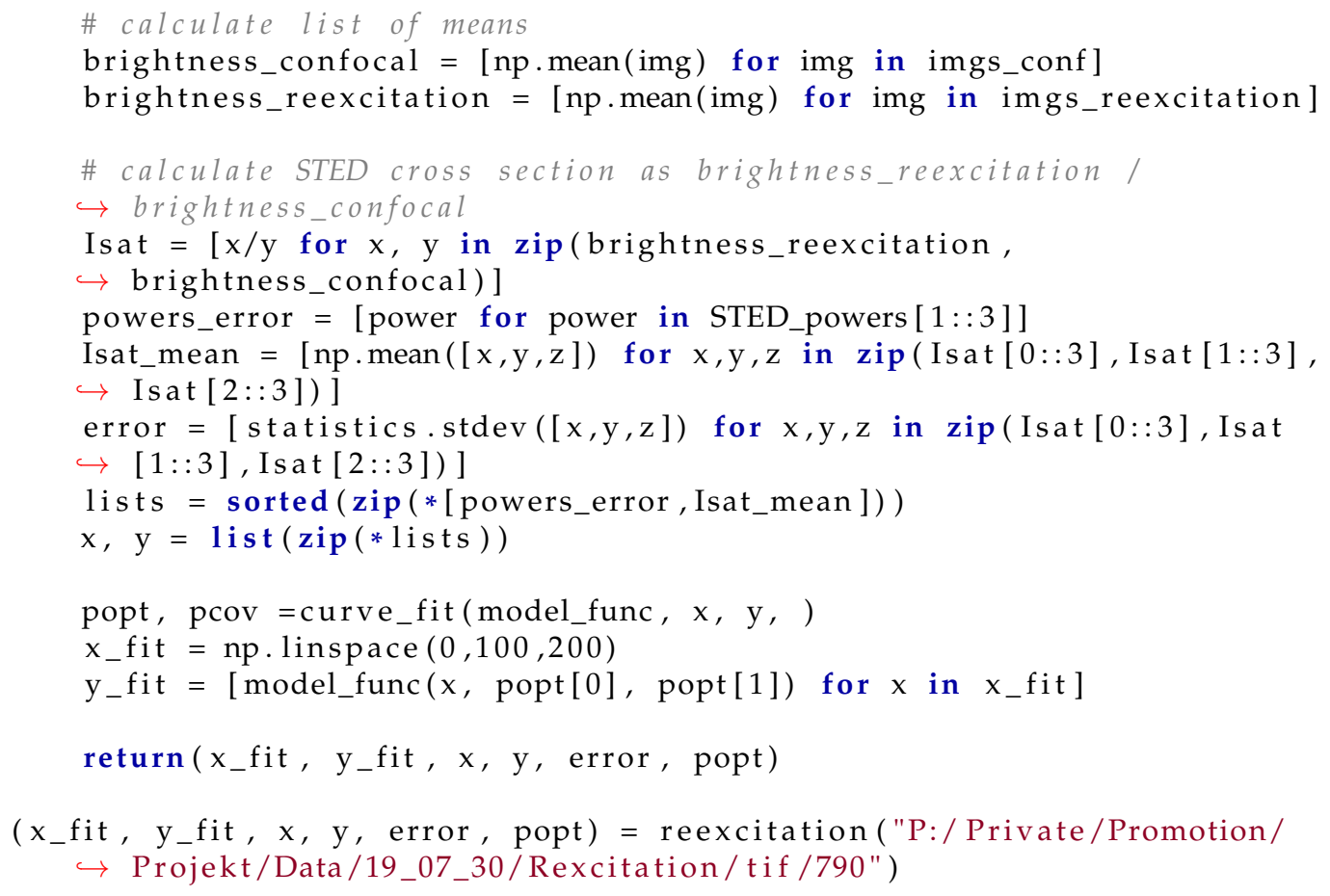

\section{C.4 Measuring STED Effect}

LISTING C.4: Python code for analysis of STED effect

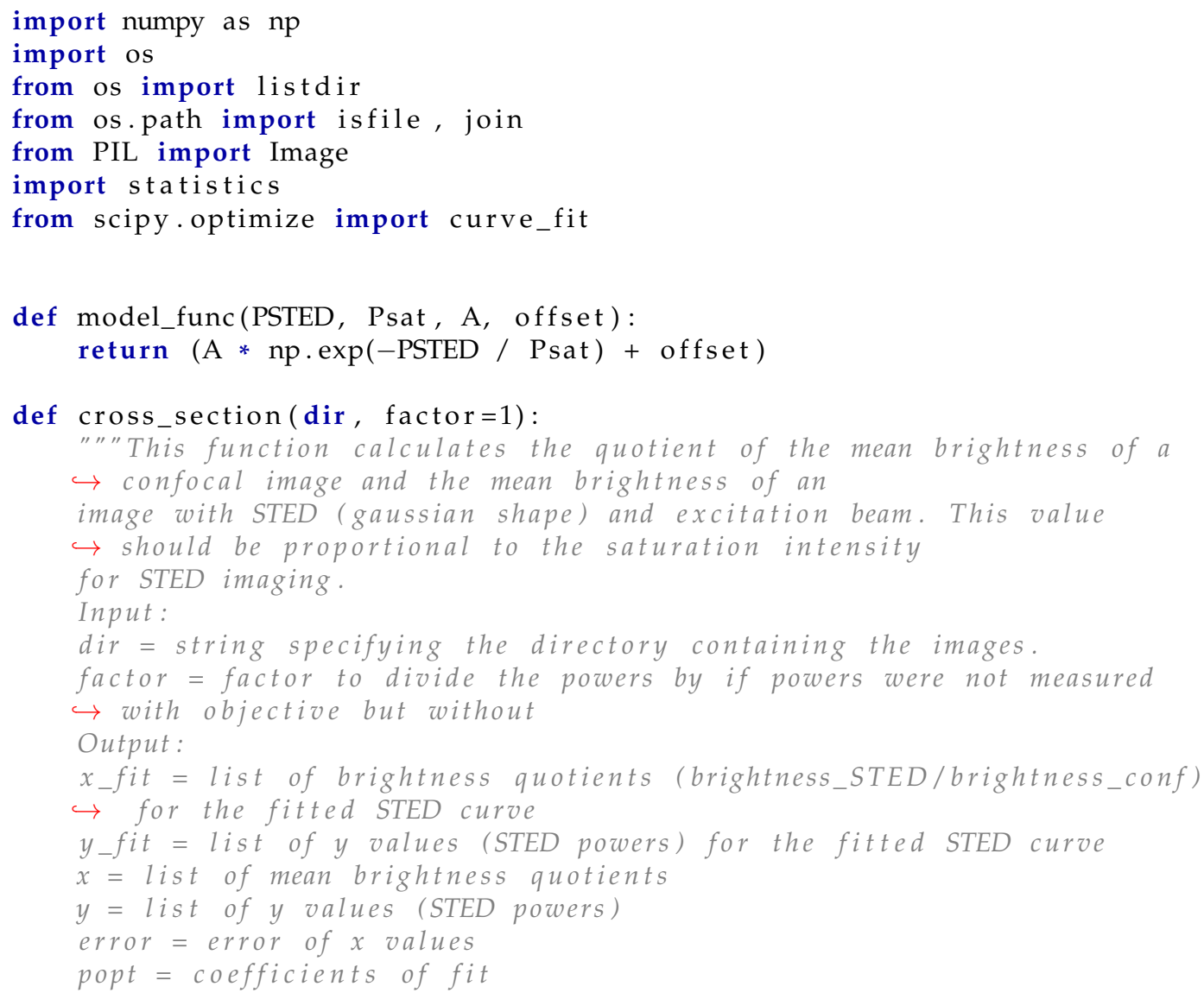




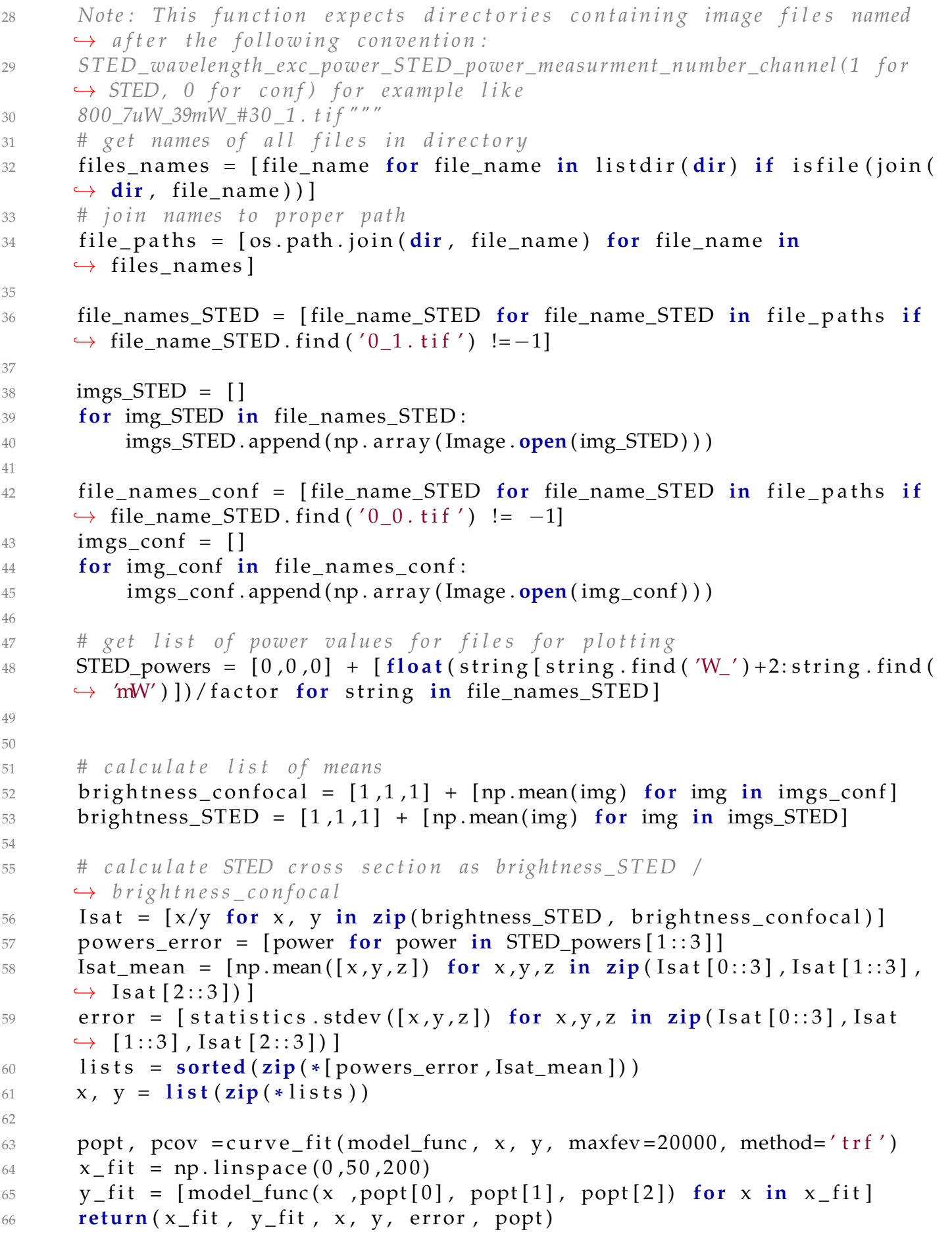

\section{C.5 FLIM}

LISTING C.5: Python code for decomposing fluorescence lifetime images

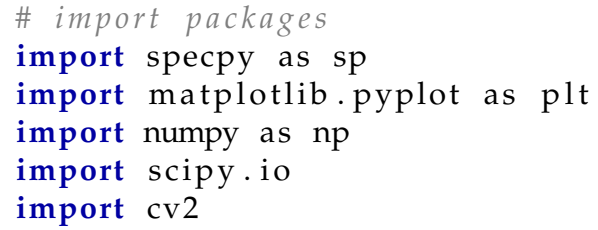




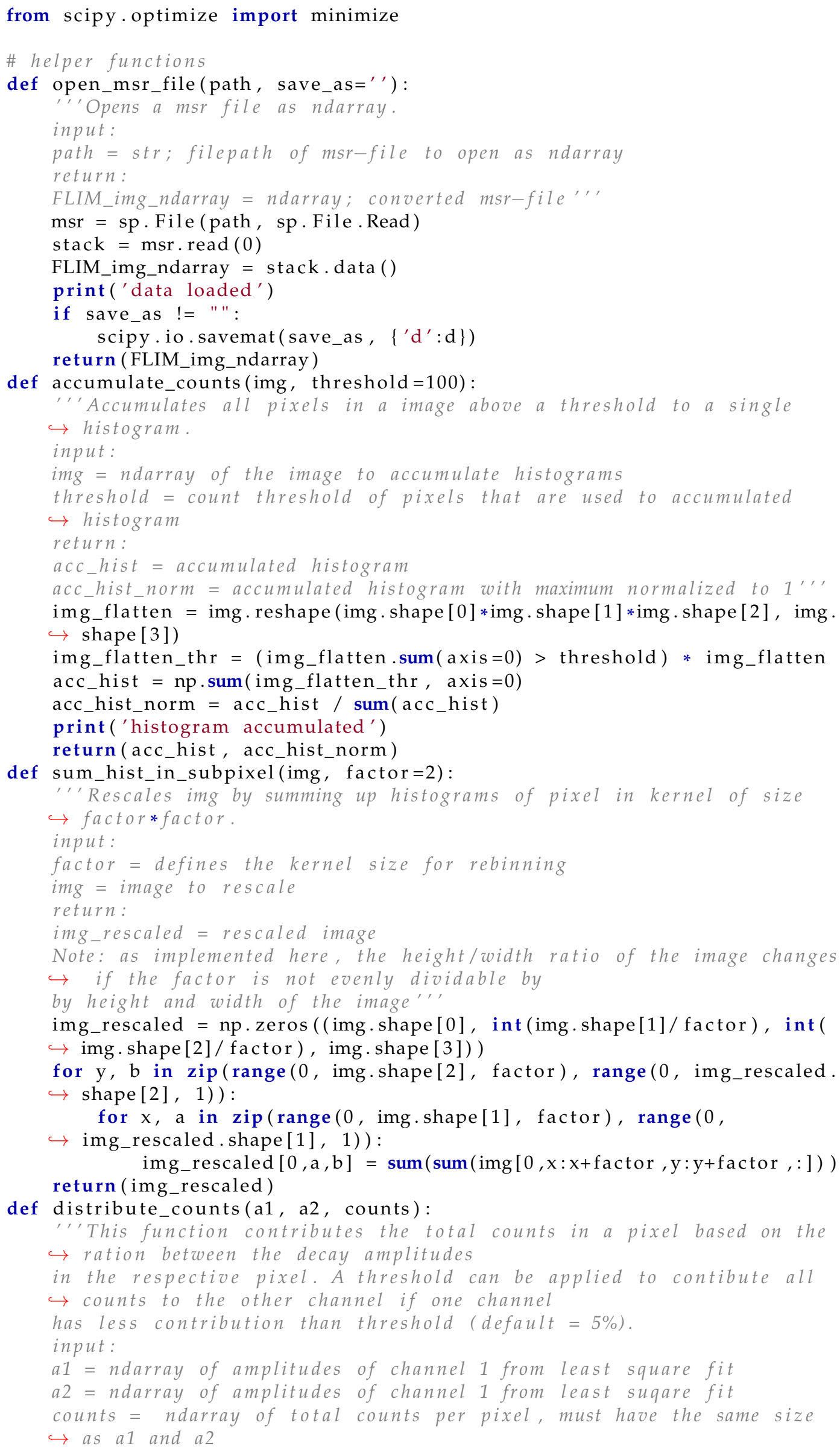




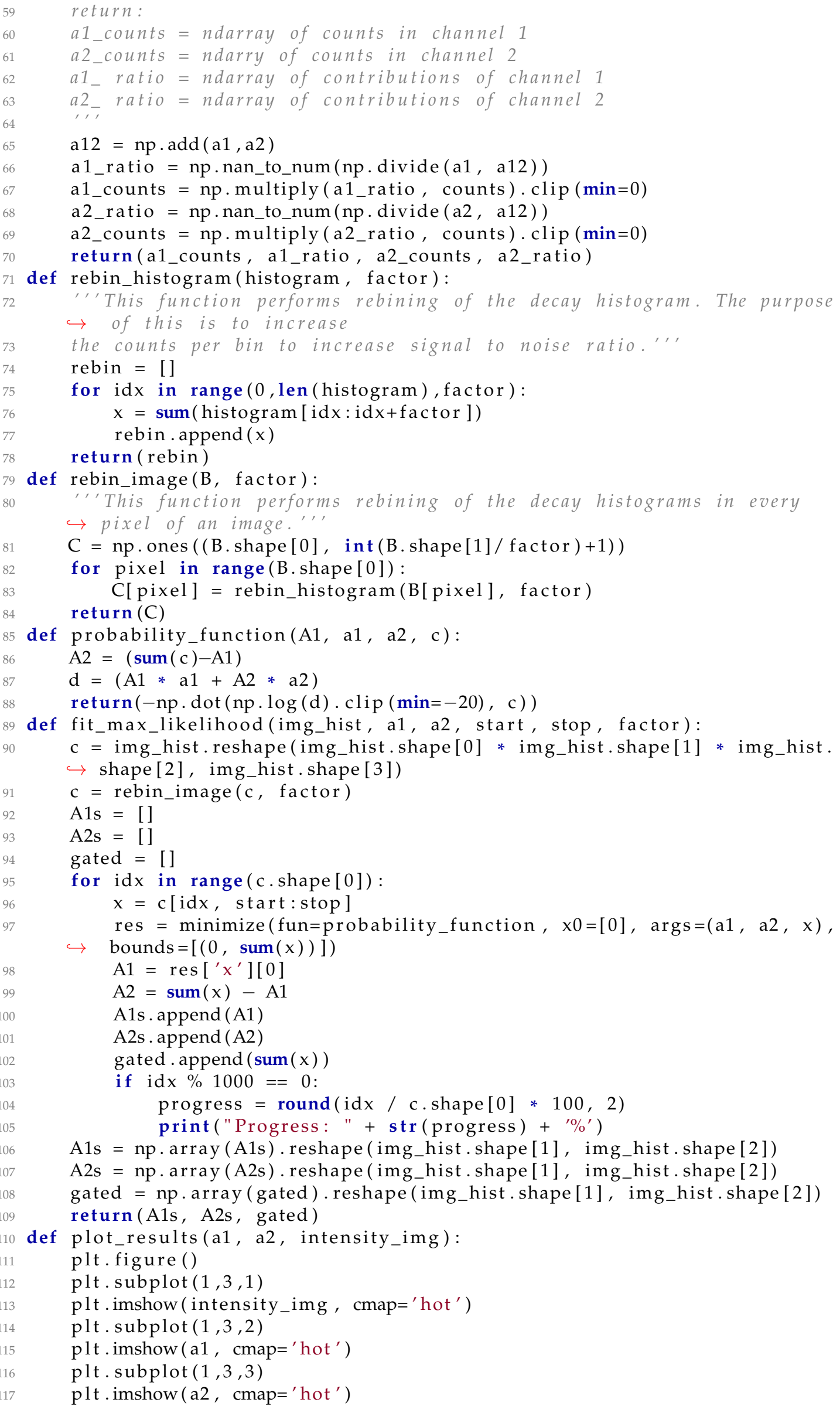




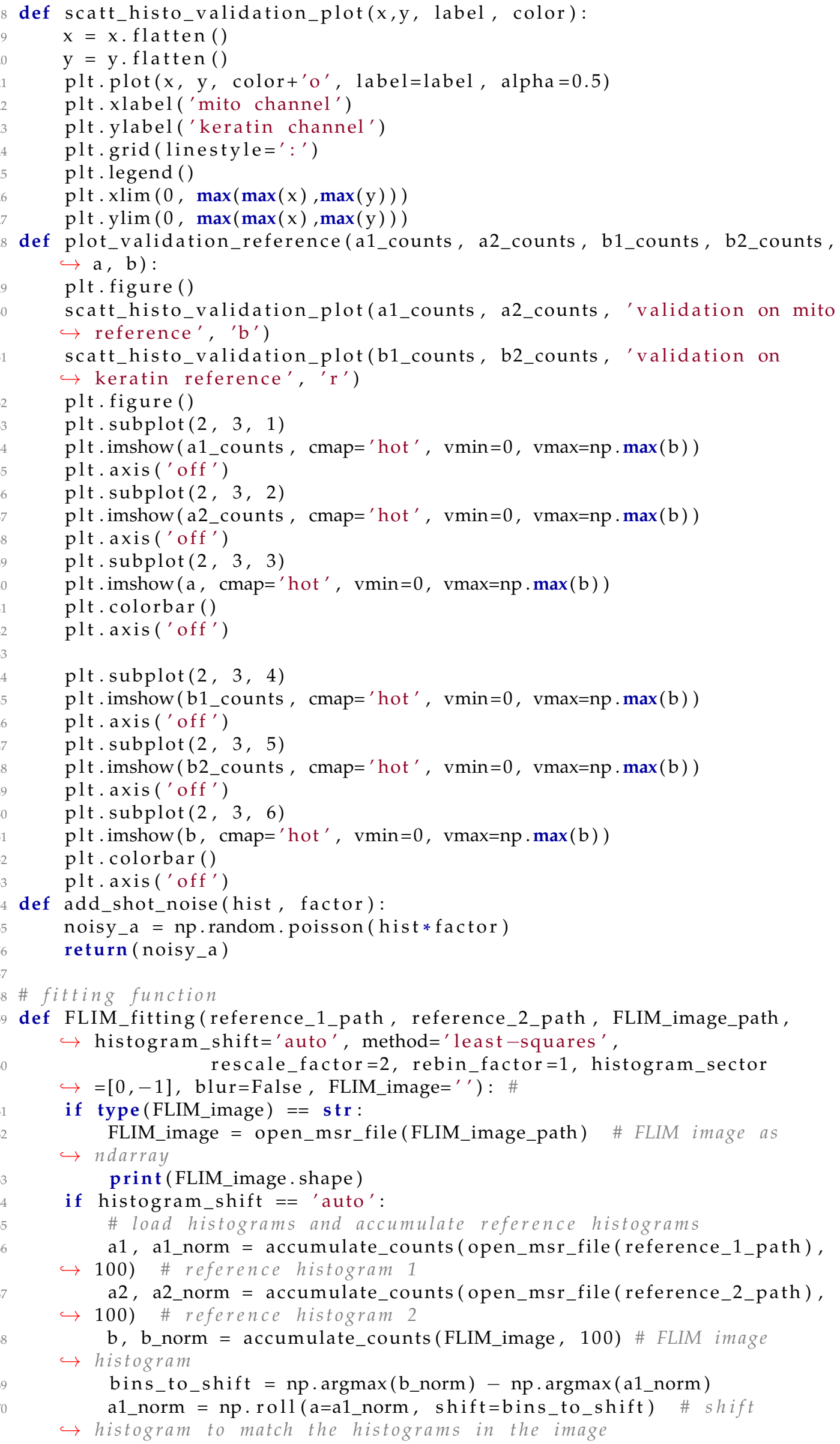




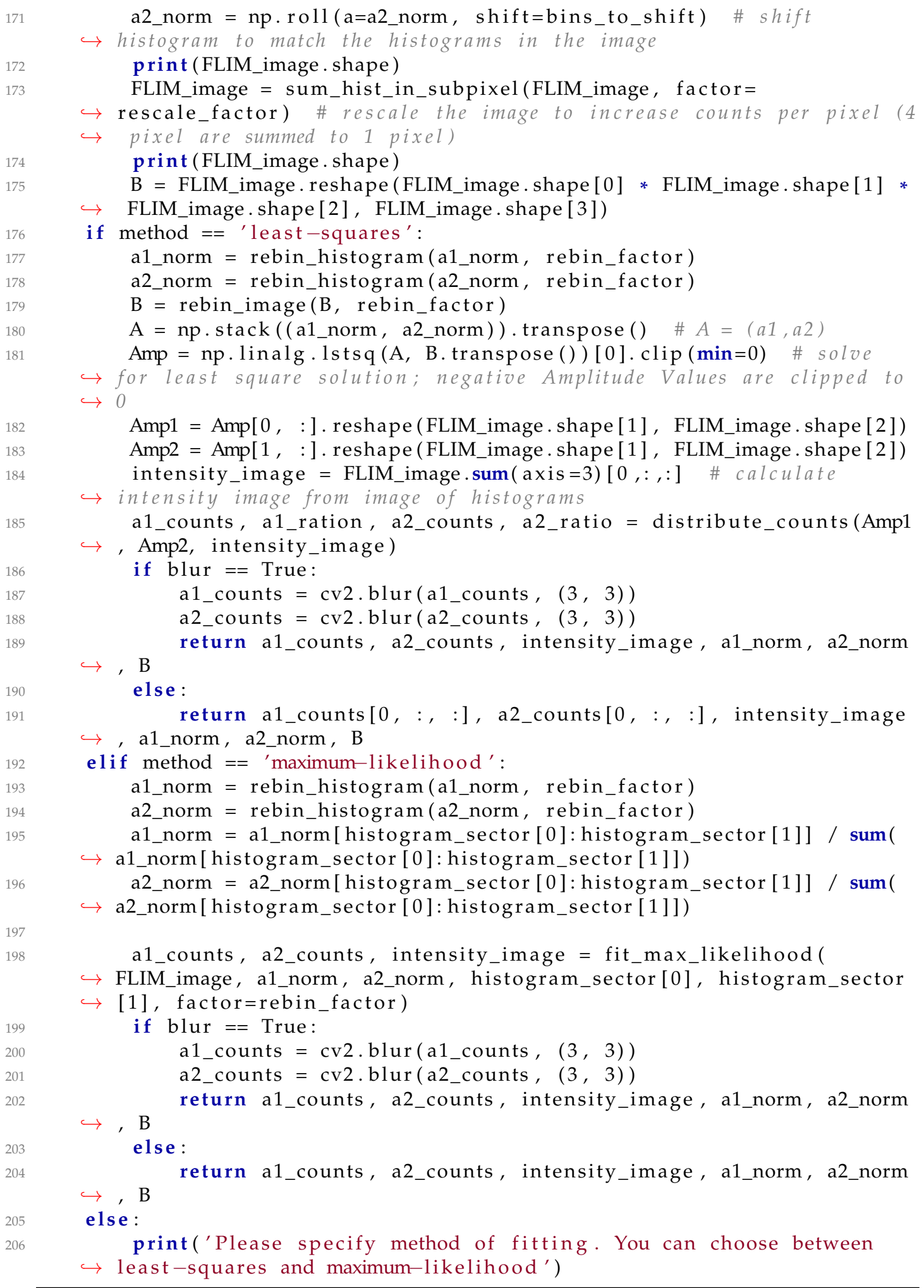




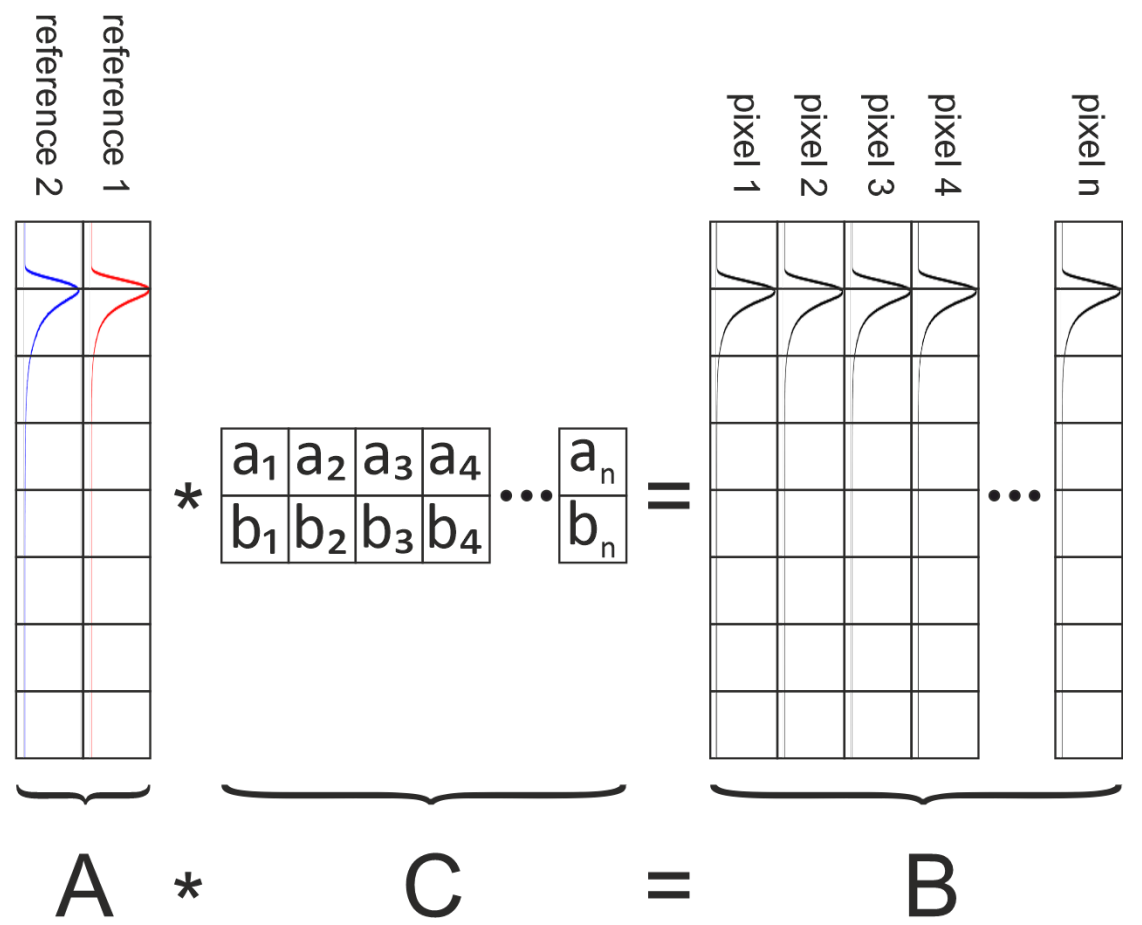

FIGURE C.1: Schematic representation of matrix equation 3.8 



\section{Appendix D}

\section{Microscopy}

\section{D.0.1 Microscope Characterization}

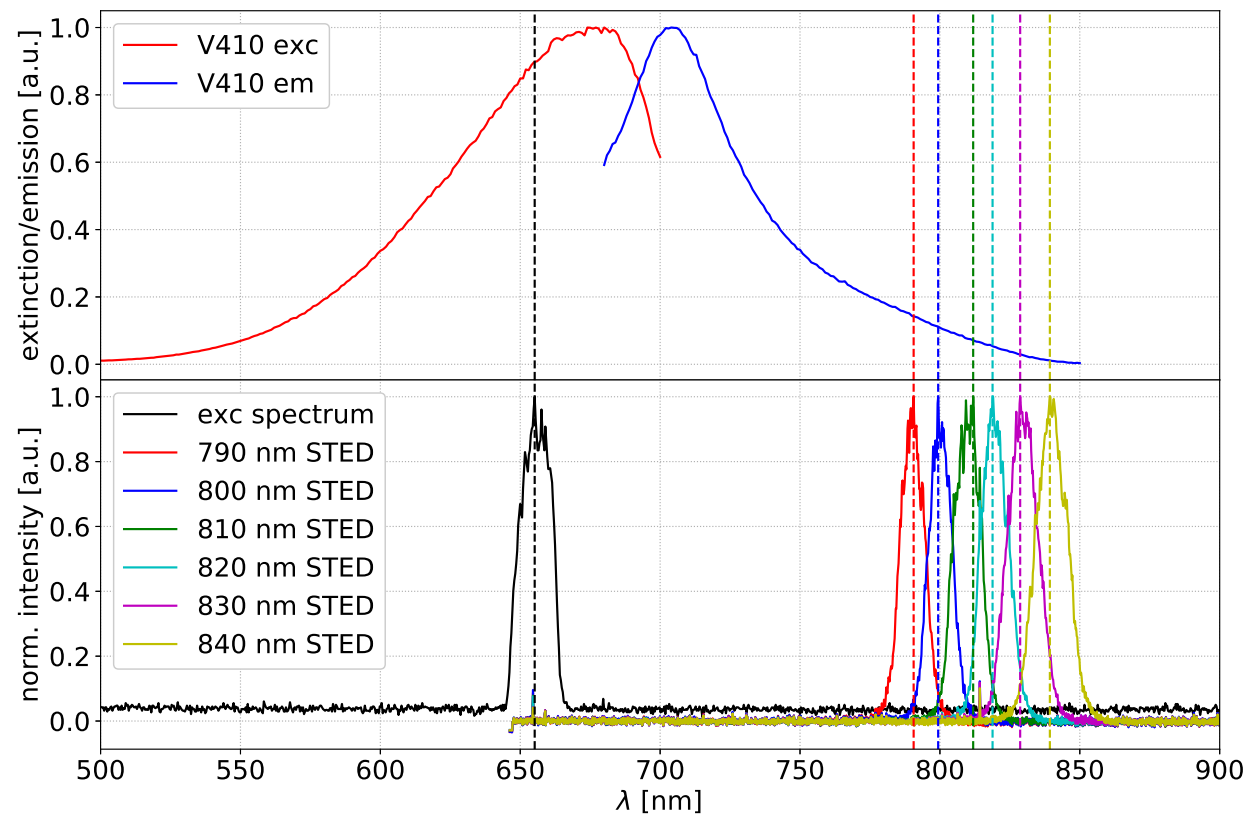

FIGURE D.1: Comparison between the excitation (top, red) and emission (top, blue) spectra of V410 with the excitation and STED spectra (bottom) of the fluorescence microscope used for imaging experiments. 

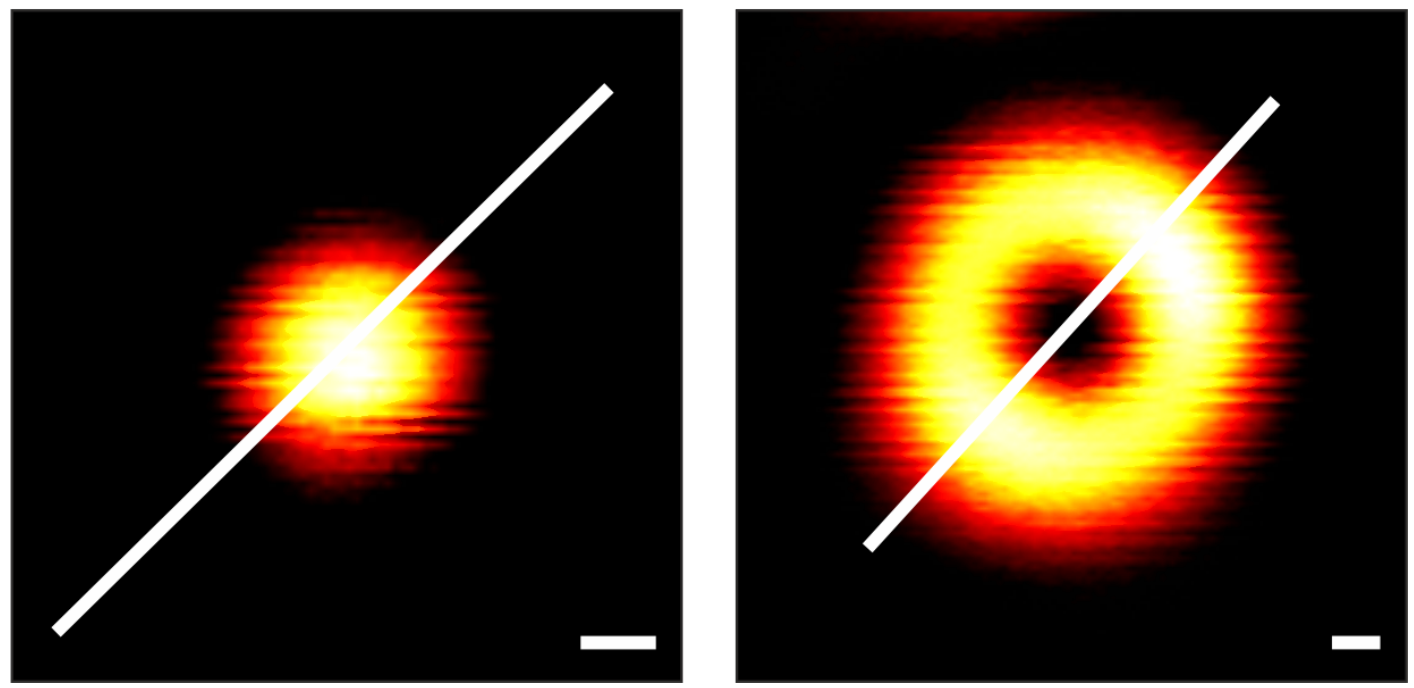

(A) Excitation and STED point spread functions

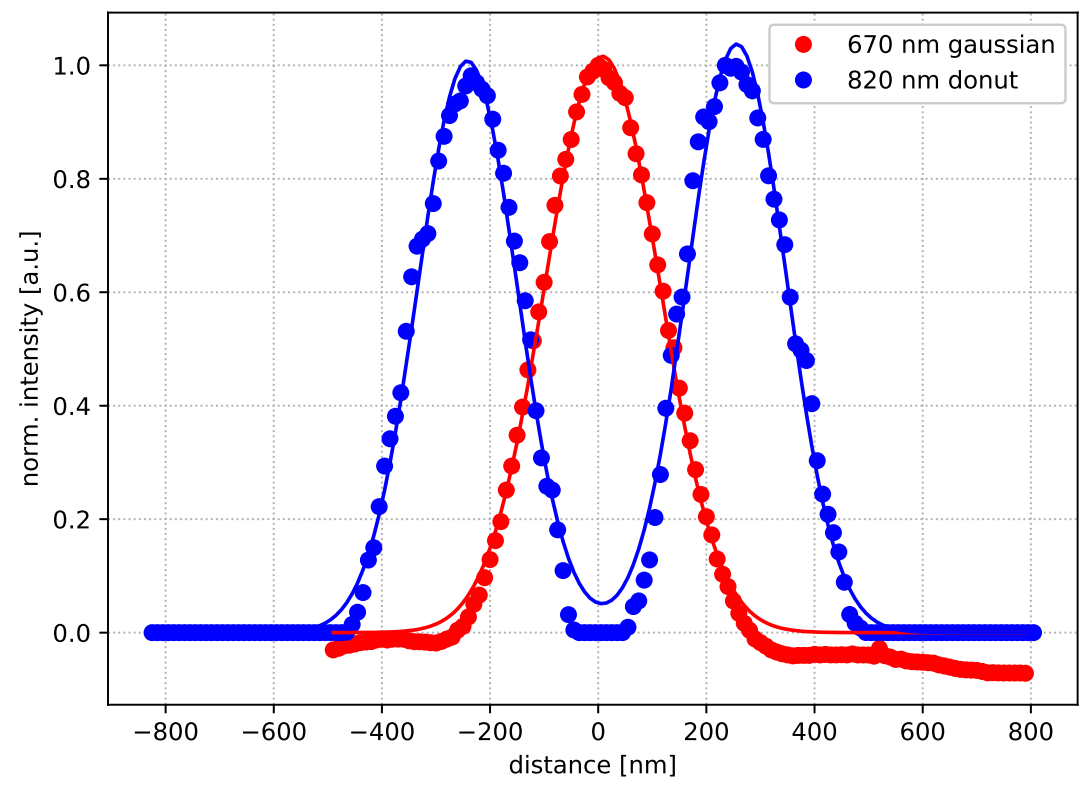

(B) Excitation and STED line profiles

FIGURE D.2: (A) Excitation (left) and STED (right) point spread functions of the commercial QUAD scanning fluorescence microscope as measured on a gold bead sample. The white lines indicate the positions where line profiles were drawn. (B) Excitation (red) and STED (blue) line profiles. The excitation line profile was fitted using a single Gaussian profile. The STED line profile was fitted using the sum of two Gaussian profiles. Scale bars: $100 \mathrm{~nm}$.

\section{D.0.2 Imaging Parameters}

All imaging parameters used to record the images shown in this work are summarized in table D.1. In order to determine the areas for the excitation and STED beams, the reflection on gold beads was measured. The profiles were fitted with a single gaussian function in case of the excitation beam and a sum of two Gaussian 
functions in case of the STED beam (see figure D.2). The areas of the excitation and STED beams were calculated using the equations D.1 and D.2.

$$
\begin{gathered}
A_{\mathrm{Exc}}=\pi *\left(\frac{F W H M_{\text {Gauss }}}{2}\right)^{2} \\
A_{\mathrm{STED}}=2 * \pi * R_{\text {donut }} * F W H M_{\text {donut }}
\end{gathered}
$$

The light intensities were calculated using equation D.3.

$$
I=\frac{P}{A}
$$

The light dose applied to the sample during imaging was calculated using equation D.4.

$$
H=\frac{P * \text { Dwelltime }}{\text { Pixelsize } * \text { Pixelsize }}
$$




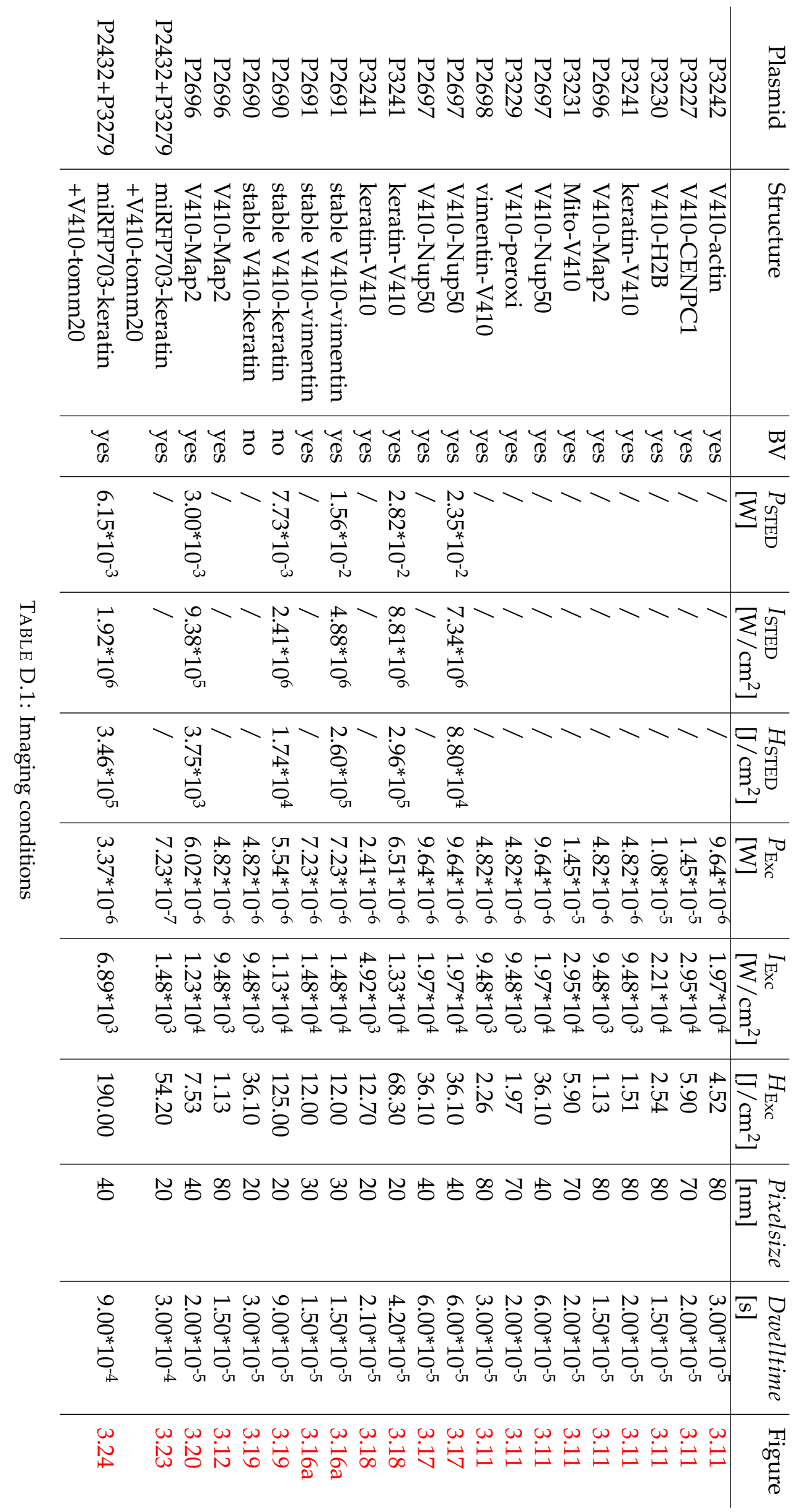




\section{D.0.3 Line Profiles}

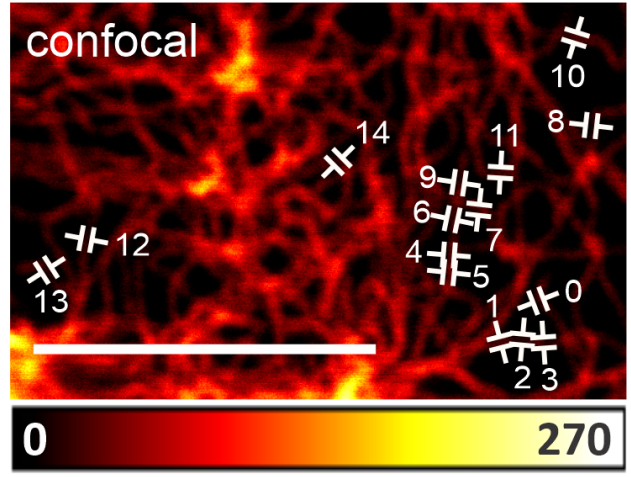

(A) Confocal image

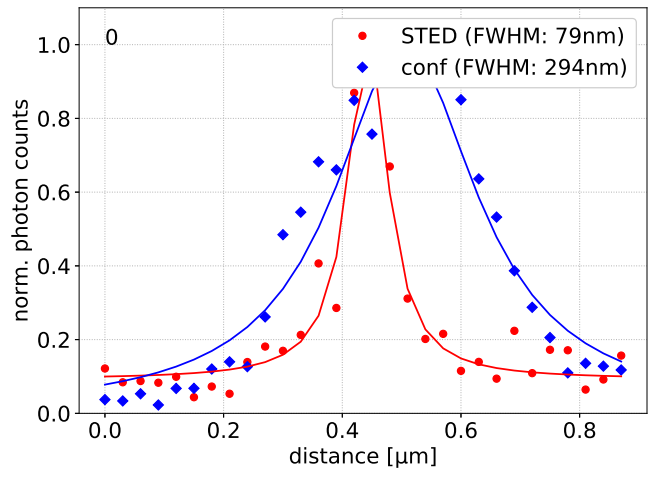

(C) Line profile 0

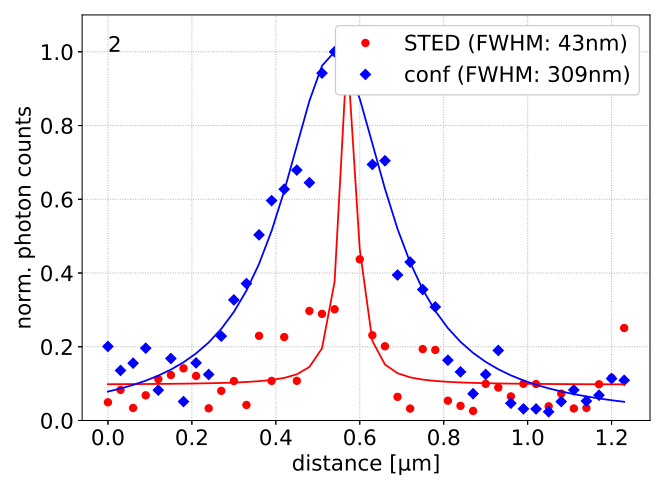

(E) Line profile 2

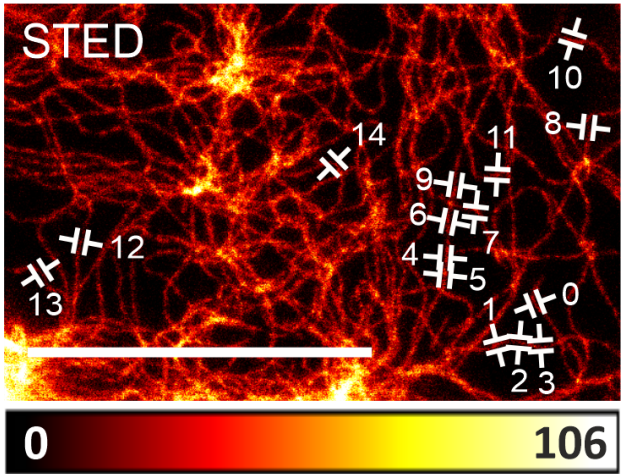

(B) STED image

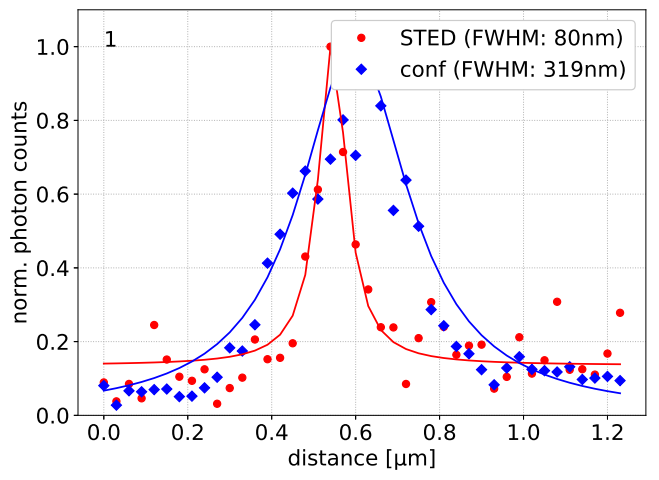

(D) Line profile 1

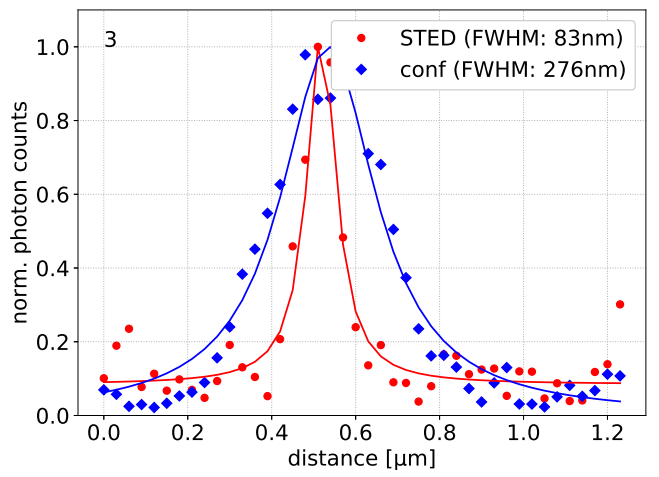

(F) Line profile 3 


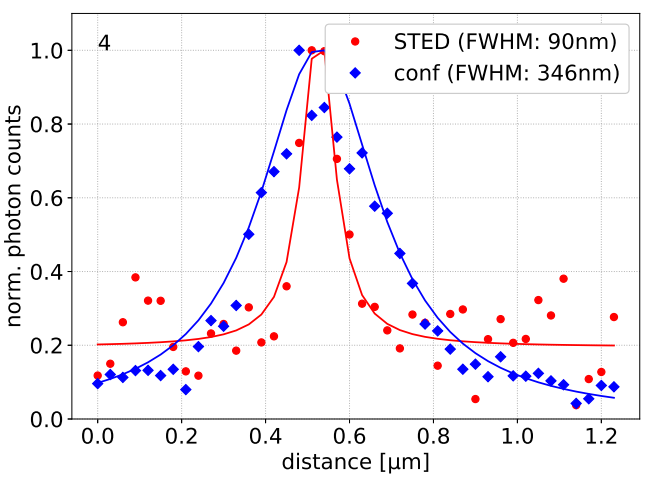

(G) Line profile 4

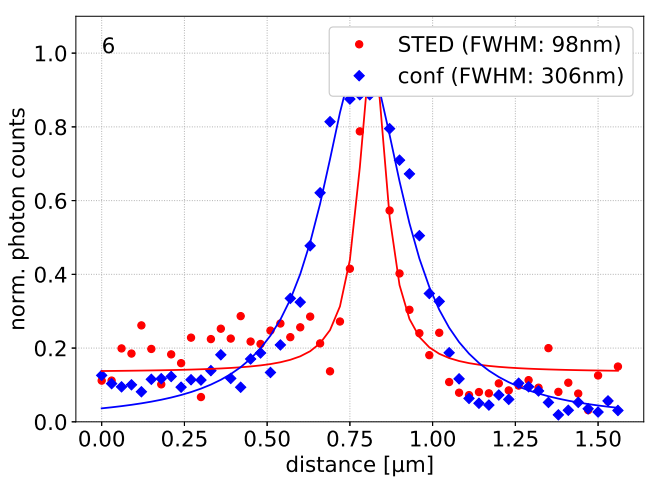

(I) Line profile 6

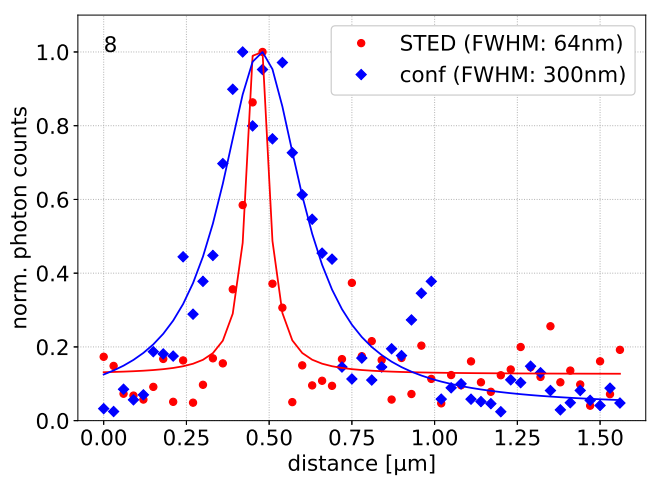

(K) Line profile 8

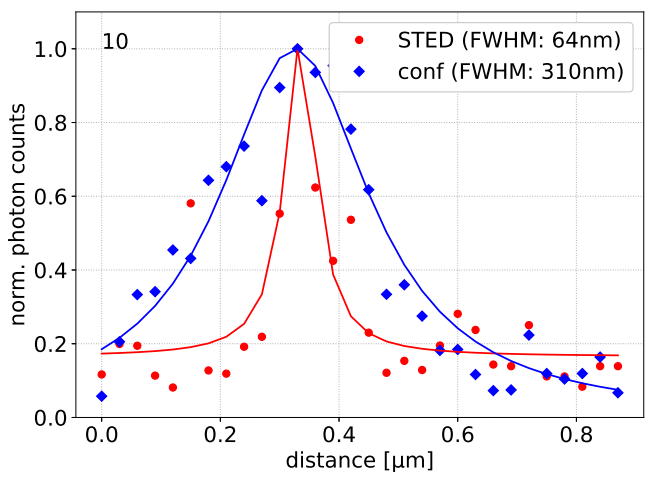

(M) Line profile 10

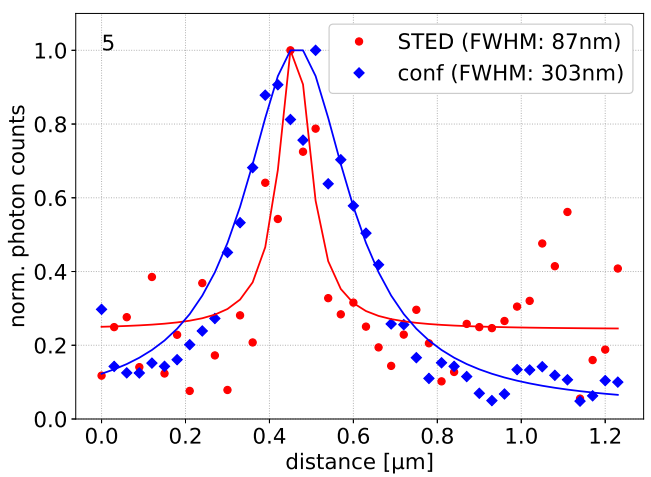

(H) Line profile 5

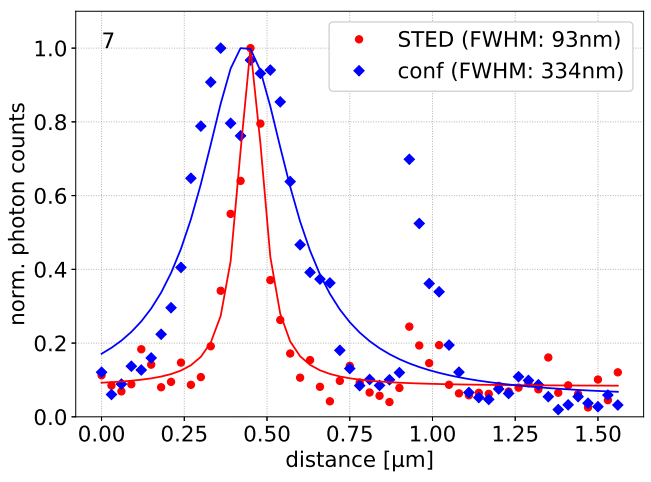

(J) Line profile 7

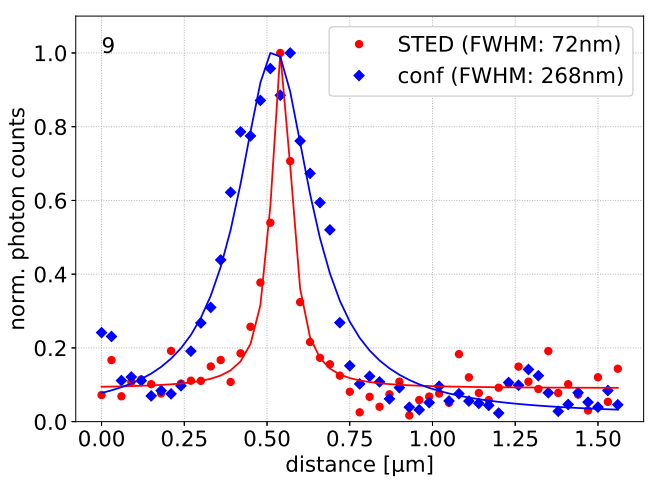

(L) Line profile 9

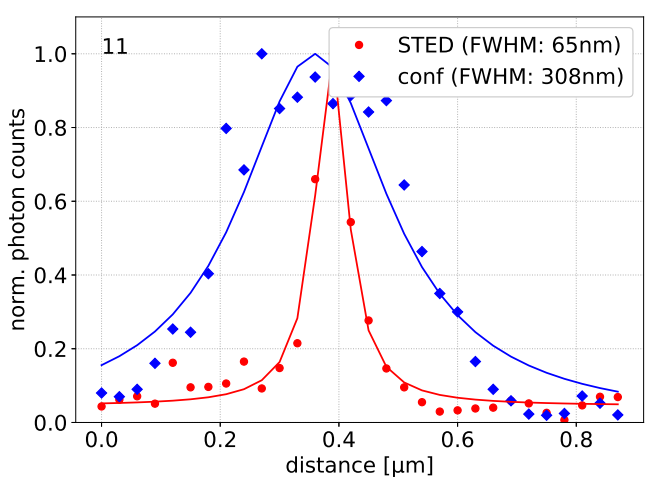

(N) Line profile 11 


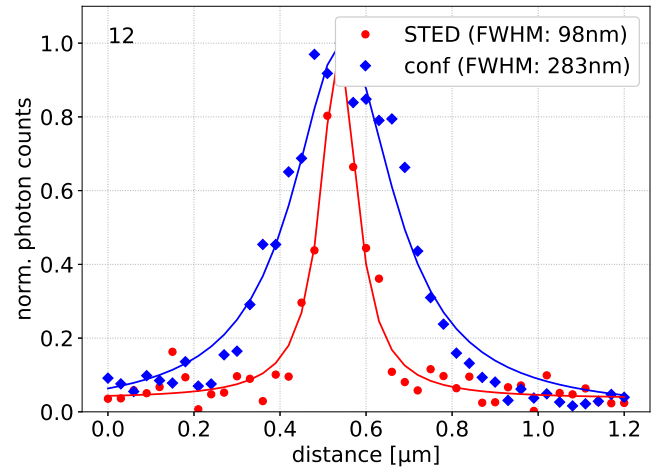

(O) Line profile 12

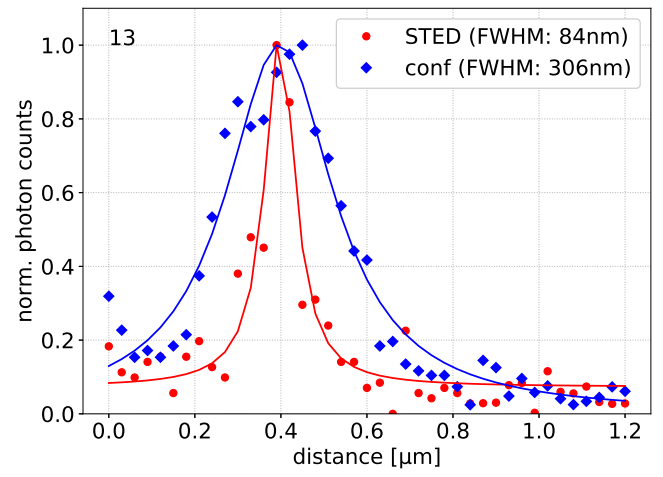

(P) Line profile 13

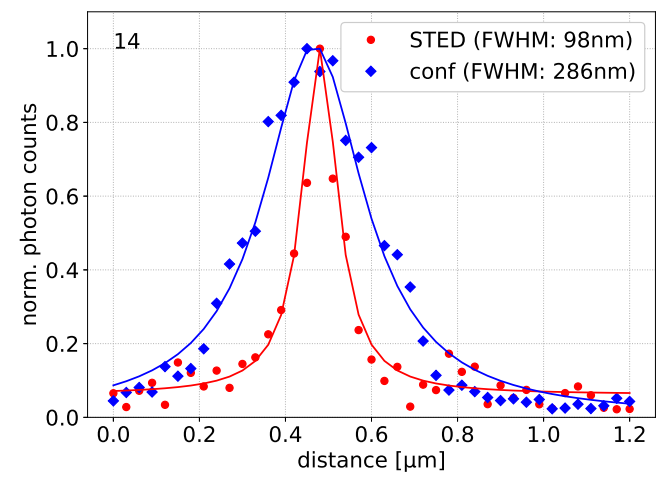

(Q) Line profile 14

FIGURE D.3: Line profiles of confocal and STED images of genome edited U2OS CRISPR cell line expressing V410-vimentin fusion construct (P2690). (A) Confocal image. (B) STED image. (C) - (Q) Line profiles. Scale bars: $10 \mu \mathrm{m}$.

\begin{tabular}{l|c|l}
$\#$ & FWHM $_{\text {conf }}$ & FWHM $_{\text {STED }}$ \\
\hline \hline 0 & $294 \mathrm{~nm}$ & $79 \mathrm{~nm}$ \\
1 & $319 \mathrm{~nm}$ & $80 \mathrm{~nm}$ \\
2 & $309 \mathrm{~nm}$ & $43 \mathrm{~nm}$ \\
3 & $276 \mathrm{~nm}$ & $83 \mathrm{~nm}$ \\
4 & $346 \mathrm{~nm}$ & $90 \mathrm{~nm}$ \\
5 & $303 \mathrm{~nm}$ & $87 \mathrm{~nm}$ \\
6 & $306 \mathrm{~nm}$ & $98 \mathrm{~nm}$ \\
7 & $334 \mathrm{~nm}$ & $93 \mathrm{~nm}$ \\
8 & $300 \mathrm{~nm}$ & $64 \mathrm{~nm}$ \\
9 & $268 \mathrm{~nm}$ & $72 \mathrm{~nm}$ \\
10 & $310 \mathrm{~nm}$ & $64 \mathrm{~nm}$ \\
11 & $308 \mathrm{~nm}$ & $65 \mathrm{~nm}$ \\
12 & $283 \mathrm{~nm}$ & $98 \mathrm{~nm}$ \\
13 & $306 \mathrm{~nm}$ & $84 \mathrm{~nm}$ \\
14 & $286 \mathrm{~nm}$ & $98 \mathrm{~nm}$ \\
\hline$\tilde{x}$ & $306 \mathrm{~nm}$ & $83 \mathrm{~nm}$
\end{tabular}

TABLE D.2: FWHM of line profiles of confocal and STED images 


\section{D.0.4 Fluorescence Lifetime Imaging}

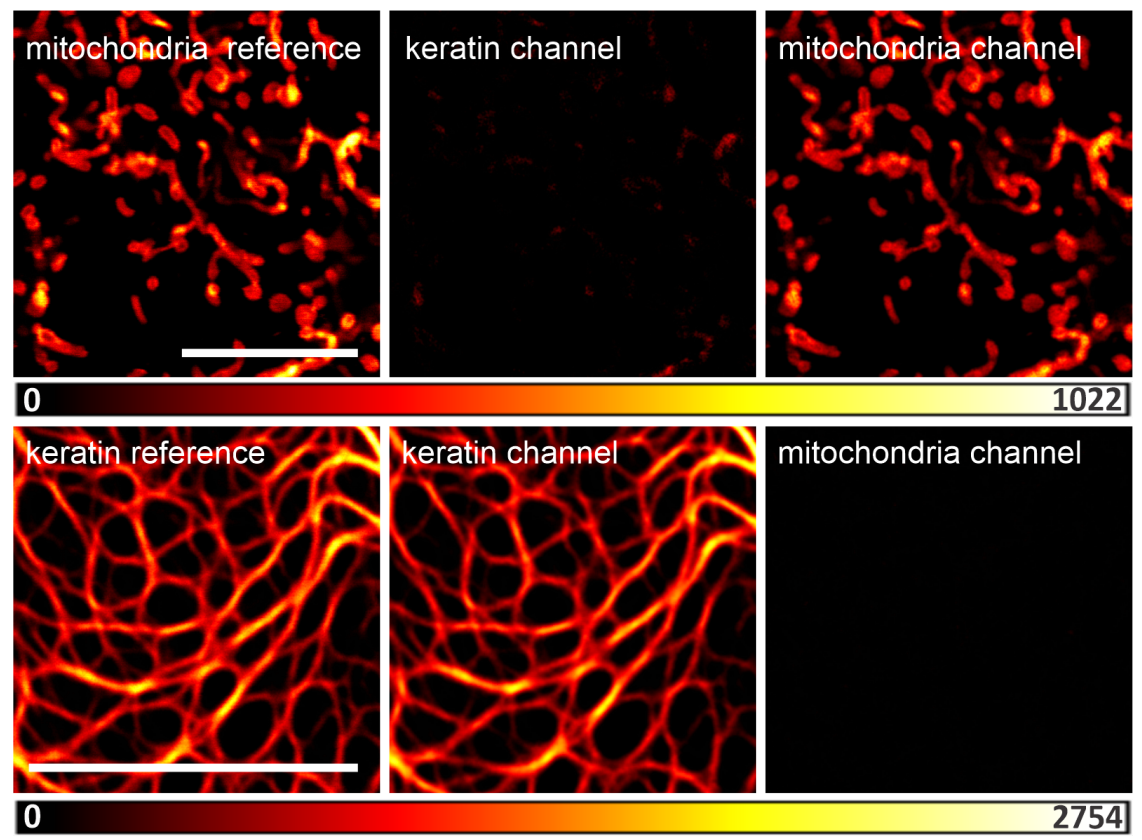

FIGURE D.4: Decomposition of reference FLIM images with maximum likelihood algorithm. The left images are the reference images to decompose, the middle and right images show the counts in the respective channels after decomposition. Scale bar: $10 \mu \mathrm{m}$.
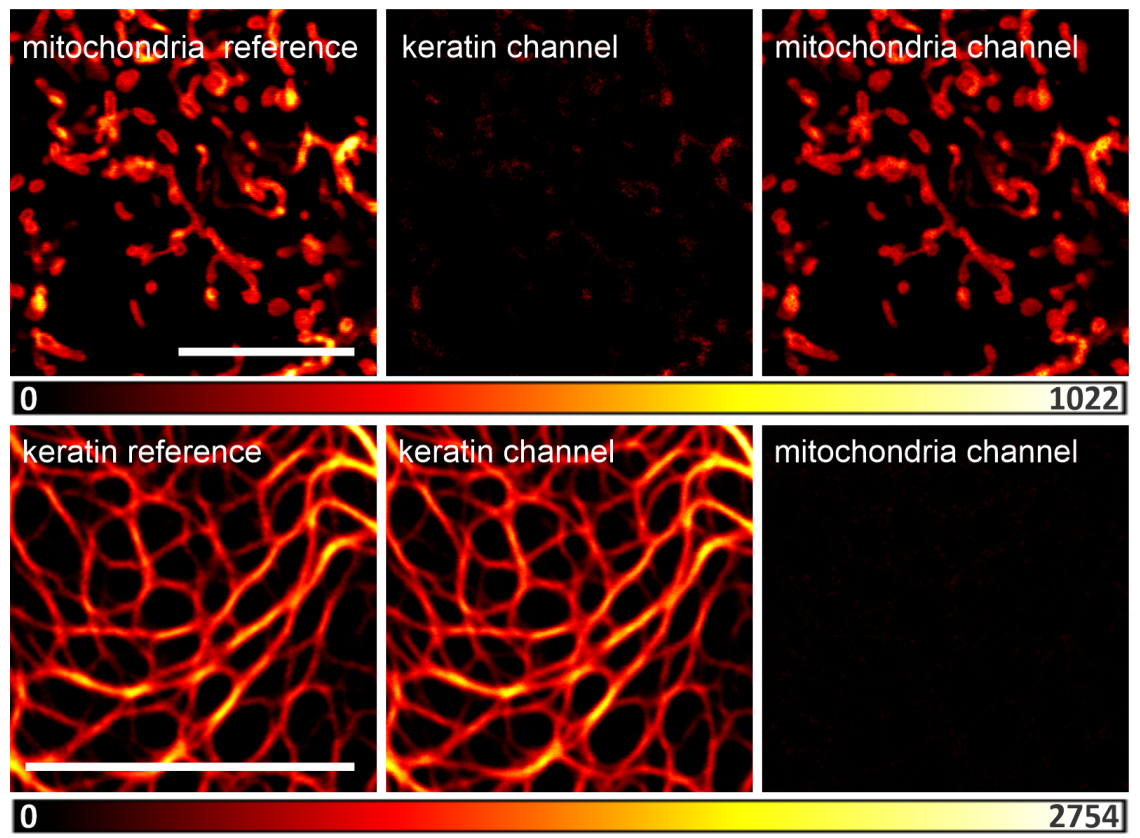

FIGURE D.5: Decomposition of reference FLIM images with least squares algorithm. The left images are the reference images to decompose, the middle and right images show the counts in the respective channels after decomposition. Scale bar: $10 \mu \mathrm{m}$. 


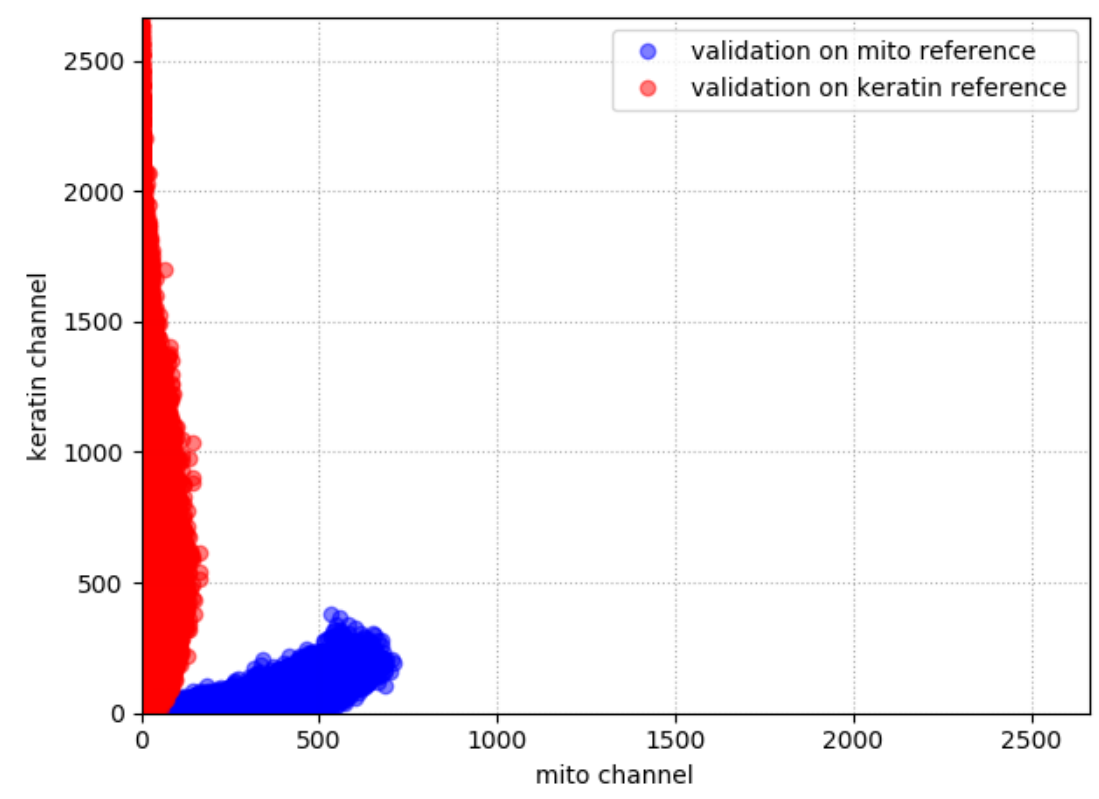

(A) Plot of count distribution maximum likelihood method

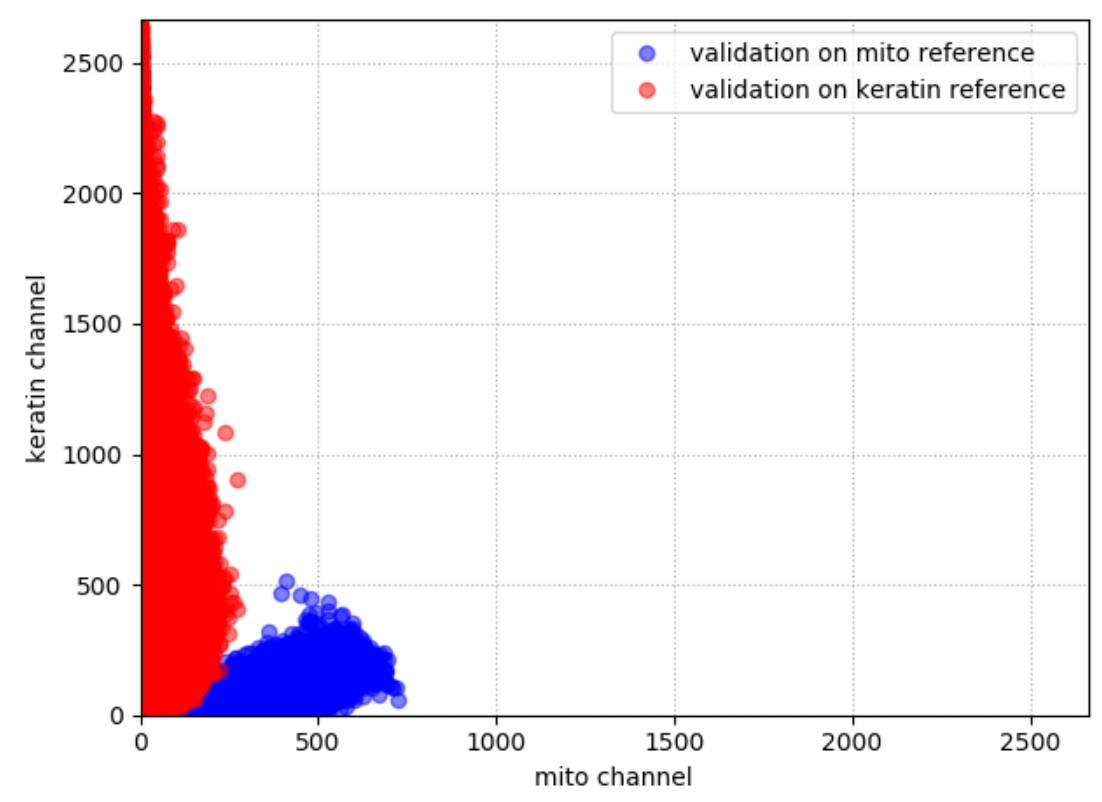

(B) Plot of count distribution least squares method

FIGURE D.6: Count distributions from decomposition of reference FLIM images. (A) Count distribution from figure D.4 and (B) count distribution from figure D.5. 


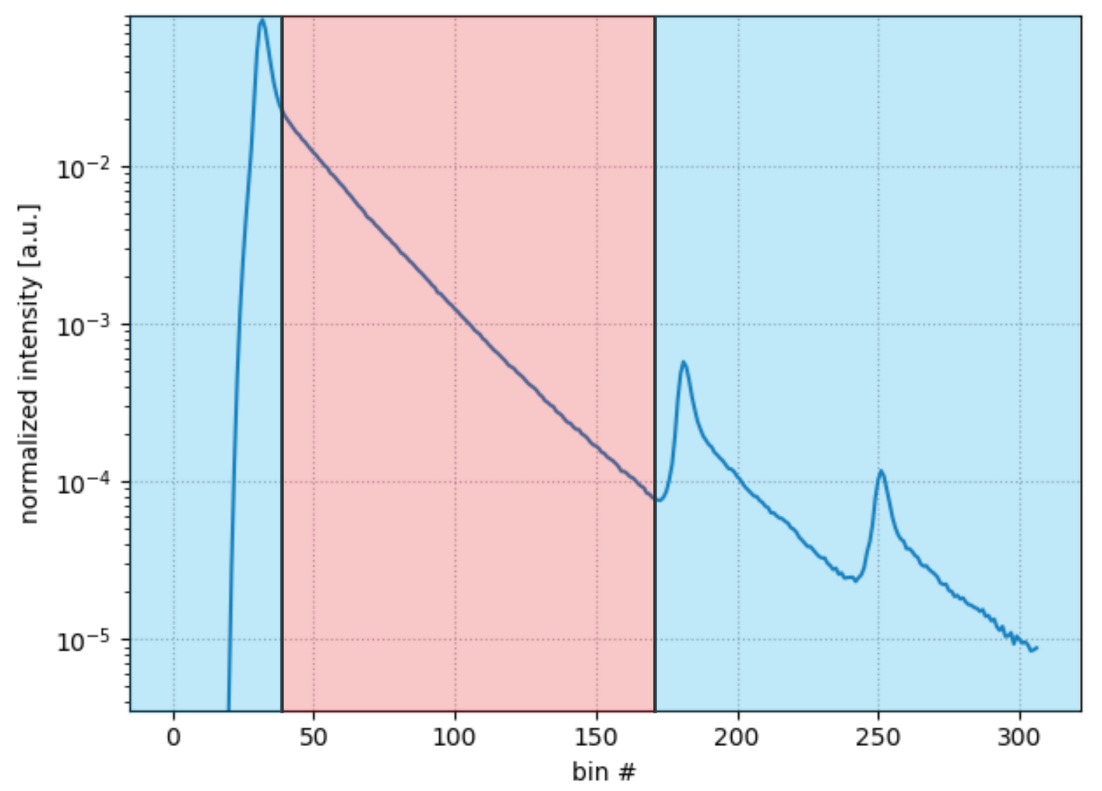

FIGURE D.7: Accumulated decay histogram FLIM-STED. The bins in the red area were used for image decomposition while the bins in the blue areas were discarded. 
Appendix E

\section{Biophysical Characterization}

E.0.1 Flow Cytometry Measurements 


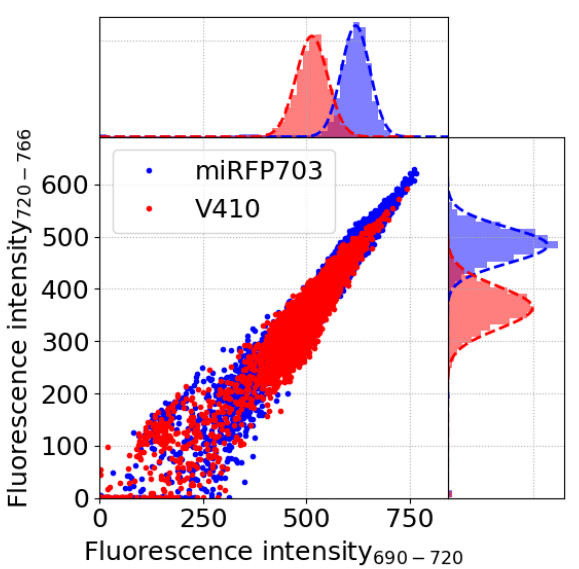

(A) $0 \mu \mathrm{MBV}$

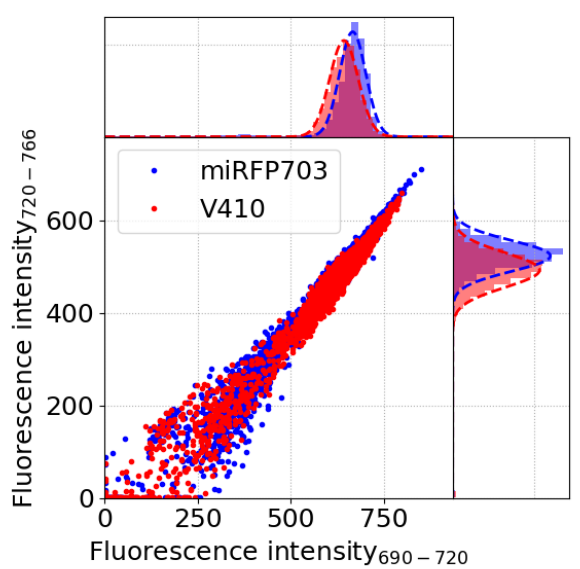

(B) $5 \mu \mathrm{M} \mathrm{BV}$

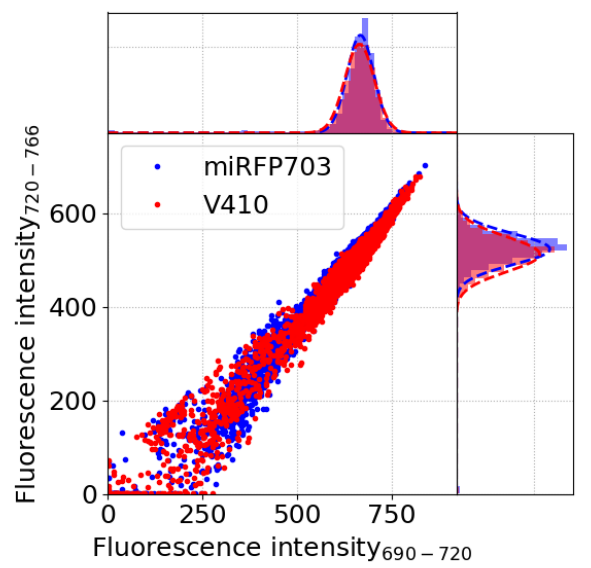

(C) $25 \mu \mathrm{MBV}$

FIGURE E.1: Flow cytometry data of stable Bxb1 landing pad cell lines expressing the fusion constructs (P2690) keratin-V410-P2A-mEGFP (red) and (P2432) keratin-miRFP703-P2A-mEGFP (blue), respectively, after incubation with (A) $0 \mu \mathrm{M} \mathrm{BV}$, (B) $5 \mu \mathrm{M} \mathrm{BV}$ and (C) $25 \mu \mathrm{M} \mathrm{BV}$ for $2 \mathrm{~h}$. The fluorescence intensity in the detection channel from 720 to $766 \mathrm{~nm}$ is plotted against the fluorescence intensity in the detection channel from 690 to $720 \mathrm{~nm}$. The distributions in both channels were fitted in Python using a Gaussian model. The measurements were performed in replicates of $n=3$ with 10000 cells for each replicate. 


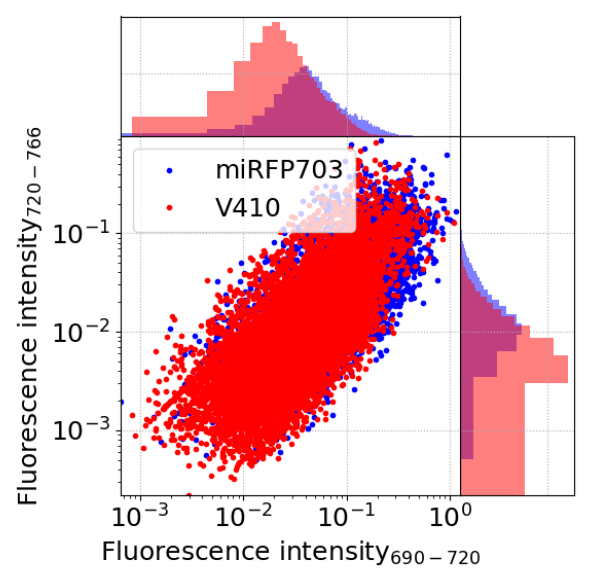

(A) $0 \mu \mathrm{M} \mathrm{BV}$

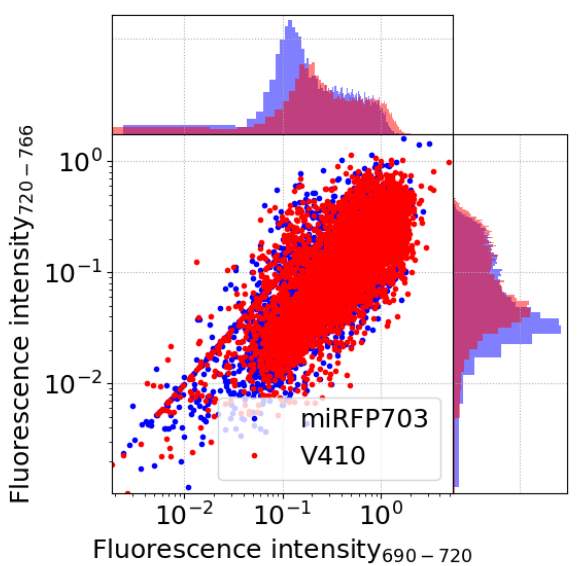

(C) $25 \mu \mathrm{M} \mathrm{BV}$

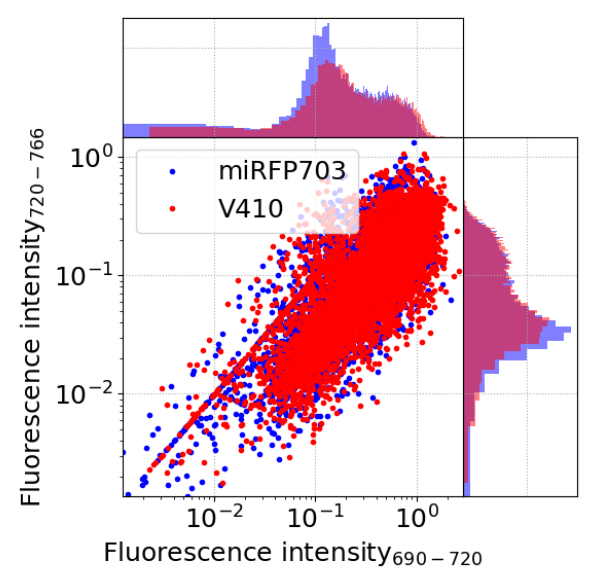

(B) $5 \mu \mathrm{M} \mathrm{BV}$

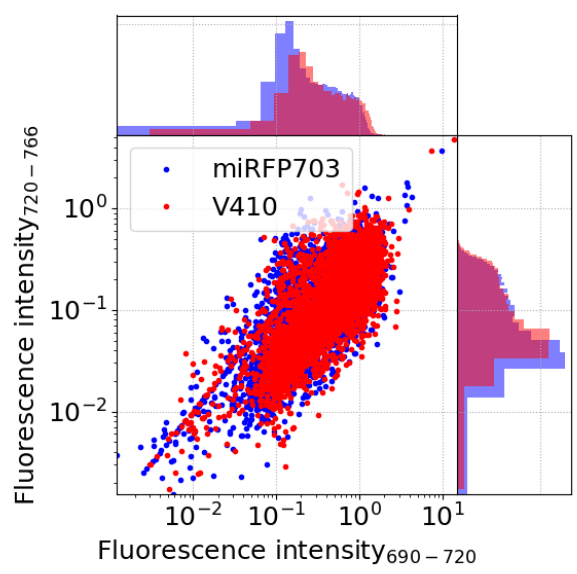

(D) $100 \mu \mathrm{M} \mathrm{BV}$

FIGURE E.2: Flow cytometry data of HeLa cells transiently expressing the fusion constructs (P3319) V410-P2A-mEGFP (orange) and (P3320) miRFP703-P2A-mEGFP (blue) after incubation with (A) $0 \mu \mathrm{M}$ BV, (B) $5 \mu \mathrm{M} \mathrm{BV}$, (C) $25 \mu \mathrm{M}$ and (D) $100 \mu \mathrm{M}$ BV for $2 \mathrm{~h}$. The fluorescence intensity in the detection channel from 720 to $766 \mathrm{~nm}$ is plotted against the fluorescence intensity in the detection channel from 690 to $720 \mathrm{~nm}$. NIR fluorescence was normalized to mEGFP fluorescence to account for variations in transfection efficiency. The measurements were performed in replicates of $\mathrm{n}=5$ with 10000 cells for each replicate. 



\section{Bibliography}

[1] E. Abbe. "Beiträge zur Theorie des Mikroskops und der mikroskopischen Wahrnehmung". In: Archiv für Mikroskopische Anatomie 9.1 (1873), pp. 413468. ISSN: 0176-7364. DOI: 10.1007/BF02956173.

[2] A. Asokan and M. J. Cho. "Exploitation of intracellular $\mathrm{pH}$ gradients in the cellular delivery of macromolecules". In: Journal of pharmaceutical sciences 91.4 (2002), pp. 903-913.

[3] M. E. Auldridge and K. T. Forest. "Bacterial phytochromes: more than meets the light". In: Critical reviews in biochemistry and molecular biology 46.1 (2011), pp. 67-88. DOI: 10.3109/10409238.2010.546389.

[4] M. E. Auldridge et al. "Structure-guided engineering enhances a phytochromebased infrared fluorescent protein". In: Journal of Biological Chemistry 287.10 (2012), pp. 7000-7009.

[5] F. Balzarotti et al. "Nanometer resolution imaging and tracking of fluorescent molecules with minimal photon fluxes". In: Science 355.6325 (2017), pp. 606612.

[6] D. Bellini and M. Z. Papiz. "Structure of a bacteriophytochrome and lightstimulated protomer swapping with a gene repressor". In: Structure 20.8 (2012), pp. 1436-1446.

[7] D. M. Benson et al. "Digital imaging fluorescence microscopy: spatial heterogeneity of photobleaching rate constants in individual cells." In: The Journal of cell biology 100.4 (1985), pp. 1309-1323.

[8] M. J. Betts and R. B. Russell. "Amino acid properties and consequences of substitutions". In: Bioinformatics for geneticists 317 (2003), p. 289.

[9] E. Betzig et al. "Imaging intracellular fluorescent proteins at nanometer resolution". In: Science 313.5793 (2006), pp. 1642-1645.

[10] E. H. Beutner. "Immunofluorescent staining: the fluorescent antibody method". In: Bacteriological reviews 25.1 (1961), p. 49.

[11] S. H. Bhoo et al. "Bacteriophytochromes are photochromic histidine kinases using a biliverdin chromophore". In: Nature 414.6865 (2001), pp. 776-779. ISSN: 1476-4687. DOI: 10.1038/414776a.

[12] B. H. Billing and G. Lathe. "Bilirubin metabolism in jaundice". In: The American journal of medicine 24.1 (1958), pp. 111-121.

[13] N. Blanckaert et al. "The fate of bilirubin-IX $\alpha$ glucuronide in cholestasis and during storage in vitro. Intramolecular rearrangement to positional isomers of glucuronic acid". In: Biochemical Journal 171.1 (1978), pp. 203-214.

[14] M. Booth et al. "Aberrations and adaptive optics in super-resolution microscopy". In: Microscopy 64.4 (2015), pp. 251-261.

[15] M. D. Bordenave et al. "STED nanoscopy with wavelengths at the emission maximum". In: Journal of Physics D: Applied Physics 49.36 (2016), p. 365102. 
[16] S. Brandt et al. "The fungal phytochrome FphA from Aspergillus nidulans". In: The Journal of biological chemistry 283.50 (2008), pp. 34605-34614. DOI: 10 . 1074/jbc.M805506200.

[17] E. Braunstein. Overview of Hemolytic Anemia - Hematology and Oncology - Merck Manuals Professional Edition. 3/24/2020. URL: https://www . merckmanuals . com / professional / hematology - and - oncology / anemias - caused - by hemolysis/overview-of-hemolytic-anemia?query=Autoimmune $\% 20 \mathrm{Hemolytic} \%$ 20 Anemia.

[18] D. Buhrke et al. "Chromophore binding to two cysteines increases quantum yield of near-infrared fluorescent proteins". In: Scientific reports 9.1 (2019), p. 1866. ISSN: 2045-2322. DOI: 10.1038/s41598-018-38433-2.

[19] L. Buitinck et al. "API design for machine learning software: experiences from the scikit-learn project". In: ECML PKDD Workshop: Languages for Data Mining and Machine Learning. 2013, pp. 108-122.

[20] A. N. Butkevich et al. "Fluorescent rhodamines and fluorogenic carbopyronines for super-resolution STED microscopy in living cells". In: Angewandte Chemie International Edition 55.10 (2016), pp. 3290-3294.

[21] N. L. Catlett, O. C. Yoder, and B. G. Turgeon. "Whole-genome analysis of twocomponent signal transduction genes in fungal pathogens". In: Eukaryotic cell 2.6 (2003), pp. 1151-1161. ISSN: 1535-9778. DOI: 10 . 1128 / ec . 2 . 6 . 1151 1161.2003.

[22] A. I. Chizhik, I. Gregor, and J. Enderlein. "Quantum yield measurement in a multicolor chromophore solution using a nanocavity". In: Nano letters 13.3 (2013), pp. 1348-1351. DOI: 10.1021/nl400313z.

[23] A. I. Chizhik et al. "Nanocavity-Based Determination of Absolute Values of Photoluminescence Quantum Yields". In: ChemPhysChem 14.3 (2013), pp. 505513.

[24] G. C. Chu et al. "Heme degradation as catalyzed by a recombinant bacterial heme oxygenase $(\mathrm{Hmu} \mathrm{O})$ from Corynebacterium diphtheriae". In: Journal of Biological Chemistry 274.30 (1999), pp. 21319-21325.

[25] G. Cox. Optical imaging techniques in cell biology. CRC Press, 2012.

[26] G. Cox and C. J. Sheppard. "Practical limits of resolution in confocal and nonlinear microscopy". In: Microscopy research and technique 63.1 (2004), pp. 1822.

[27] R. J. W. Cremlyn and R. J. Cremlyn. An introduction to organosulfur chemistry. Vol. 8. Wiley New York, 1996.

[28] F. Danielsson et al. "Vimentin diversity in health and disease". In: Cells 7.10 (2018), p. 147.

[29] L. R. De Young, K. A. Dill, and A. L. Fink. “Aggregation and denaturation of apomyoglobin in aqueous urea solutions". In: Biochemistry 32.15 (1993), pp. 3877-3886.

[30] W.-L. Ding et al. "Small monomeric and highly stable near-infrared fluorescent markers derived from the thermophilic phycobiliprotein, ApcF2". In: Biochimica et biophysica acta (2017). ISSN: 0006-3002. DOI: 10 . 1016/ j . bbamcr . 2017.08 .002 . 
[31] W. J. Dower, J. F. Miller, and C. W. Ragsdale. "High efficiency transformation of E. coli by high voltage electroporation". In: Nucleic Acids Research 16.13 (1988), pp. 6127-6145. ISSN: 0305-1048.

[32] J. M. Dudley, G. Genty, and S. Coen. "Supercontinuum generation in photonic crystal fiber". In: Reviews of modern physics 78.4 (2006), p. 1135.

[33] C Duy and J Fitter. "How aggregation and conformational scrambling of unfolded states govern fluorescence emission spectra". In: Biophysical journal 90.10 (2006), pp. 3704-3711.

[34] M. R. Eftink. "The use of fluorescence methods to monitor unfolding transitions in proteins". In: Biophysical journal 66.2 (1994), pp. 482-501.

[35] Y. Eilers et al. "MINFLUX monitors rapid molecular jumps with superior spatiotemporal resolution". In: Proceedings of the National Academy of Sciences 115.24 (2018), pp. 6117-6122.

[36] T. D. Elich and J. Chory. "Biochemical characterization of Arabidopsis wildtype and mutant phytochrome B holoproteins." In: The Plant Cell 9.12 (1997), pp. 2271-2280.

[37] J. Enderlein and R. Erdmann. "Fast fitting of multi-exponetial decay curves". In: Optics Communications 134.1-6 (1997), pp. 371-378. ISSN: 00304018. DOI: 10.1016/S0030-4018(96)00384-7.

[38] L.-O. Essen, J. Mailliet, and J. Hughes. "The structure of a complete phytochrome sensory module in the Pr ground state". In: Proceedings of the National Academy of Sciences 105.38 (2008), pp. 14709-14714.

[39] G. S. Filonov et al. "Bright and stable near-infrared fluorescent protein for in vivo imaging". In: Nature biotechnology 29.8 (2011), pp. 757-761. ISSN: 15461696. DOI: $10.1038 / \mathrm{nbt} .1918$.

[40] A. J. Fischer et al. "Multiple roles of a conserved GAF domain tyrosine residue in cyanobacterial and plant phytochromes". In: Biochemistry 44.46 (2005).

[41] A. E. Fitzpatrick et al. "Pump-dump-probe and pump-repump-probe ultrafast spectroscopy resolves cross section of an early ground state intermediate and stimulated emission in the photoreactions of the pr ground state of the cyanobacterial phytochrome cph1". In: The Journal of Physical Chemistry B 116.3 (2012), pp. 1077-1088.

[42] Fluorescent protein properties. 5/14/2018. URL: http://www . fpvis .org/FP . html.

[43] N. Frankenberg and J. Lagarias. "Biosynthesis and biological function of bilins. The Porphyrin Handbook. Chlorophylls and Bilins: Biosynthesis Structure and Degrada-tion, eds Kadish KM, Smith KM, Guillard R". In: New York, Academic Press 13 (2003), pp. 211-235.

[44] P. Gao and G. U. Nienhaus. "Precise background subtraction in stimulated emission double depletion nanoscopy". In: Optics letters 42.4 (2017), pp. 831834.

[45] P. Gao et al. "Background suppression in fluorescence nanoscopy with stimulated emission double depletion". In: Nature Photonics 11.3 (2017), p. 163.

[46] B. Gibson et al. "The distribution of bacterial doubling times in the wild". In: Proceedings of the Royal Society B: Biological Sciences 285.1880 (2018), p. 20180789. 
[47] J. Goedhart et al. "Bright cyan fluorescent protein variants identified by fluorescence lifetime screening". In: Nature methods 7.2 (2010), p. 137.

[48] G. Goldstein, I. S. Slizys, and M. W. Chase. "Studies on fluorescent antibody staining: I. Non-specific fluorescence with fluorescein-coupled sheep antirabbit globulins". In: The Journal of experimental medicine 114.1 (1961), p. 89.

[49] A. Goryaynov, J. Neuwirth, and J. Bewersdorf. "Quantitative Determination of Phototoxicity in Live Cell Super-Resolution Microscopy". In: Biophysical Journal 110.3 (2016), 487a.

[50] K. C. Gwosch et al. "MINFLUX nanoscopy delivers multicolor nanometer 3D-resolution in (living) cells". In: bioRxiv (2019), p. 734251.

[51] O. Heimstiidt. "Das fluoreszenzmikroskop". In: Zeitschrift für wissenschaftliche Mikroskopie und mikroskopische Technik 28 (1911), p. 330.

[52] S. W. Hell, M. Dyba, and S. Jakobs. "Concepts for nanoscale resolution in fluorescence microscopy". In: Current opinion in neurobiology 14.5 (2004), pp. 599 609. ISSN: 0959-4388. DOI: $10.1016 / \mathrm{j}$. conb. 2004.08.015.

[53] S. W. Hell and J. Wichmann. "Breaking the diffraction resolution limit by stimulated emission: stimulated-emission-depletion fluorescence microscopy". In: Optics letters 19.11 (1994), pp. 780-782.

[54] M. Hermann et al. "Binary recombinase systems for high-resolution conditional mutagenesis". In: Nucleic Acids Research 42.6 (2014), pp. 3894-3907. ISSN: 0305-1048. DOI: 10.1093/nar/gkt1361.

[55] H. Herrmann et al. "Characterization of distinct early assembly units of different intermediate filament proteins". In: Journal of molecular biology 286.5 (1999), pp. 1403-1420.

[56] H. Herrmann et al. "Structure and assembly properties of the intermediate filament protein vimentin: the role of its head, rod and tail domains". In: Journal of molecular biology 264.5 (1996), pp. 933-953.

[57] M. Hofmann et al. "Breaking the diffraction barrier in fluorescence microscopy at low light intensities by using reversibly photoswitchable proteins". In: Proceedings of the National Academy of Sciences 102.49 (2005), pp. 17565-17569.

[58] Y. Hontani et al. "Bright blue-shifted fluorescent proteins with Cys in the GAF domain engineered from bacterial phytochromes: Fluorescence mechanisms and excited-state dynamics". In: Scientific reports 6 (2016), p. 37362. ISSN: 20452322. DOI: $10.1038 /$ srep37362.

[59] R. Hooke. Micrographia (1665). Vol. 20. AppLife, 2014.

[60] J. Hughes et al. "A prokaryotic phytochrome". In: Nature 386.6626 (1997), p. 663. ISSN: 1476-4687. DOI: 10.1038/386663a0.

[61] I. Jansen. "Development and characterization of novel reversibly switchable red fluorescent proteins with opposing switching modes". PhD Thesis. 2019.

[62] E. Jones, T. Oliphant, P. Peterson, et al. SciPy: Open source scientific tools for Python. [Online; accessed ]. 2001-. URL: http://www . scipy . org/.

[63] A. Jozkowicz, H. Was, and J. Dulak. "Heme oxygenase-1 in tumors: is it a false friend?" In: Antioxidants \& redox signaling 9.12 (2007), pp. 2099-2117. ISSN: 1523-0864. DOI: 10.1089/ars .2007.1659. 
[64] M. Kamper. "Gerichtete Evolution und Charakterisierung von Bakteriophytochromen für die tiefrote, hochauflösende Fluoreszenzmikroskopie". PhD Thesis.

[65] M. Kamper et al. "Near-infrared STED nanoscopy with an engineered bacterial phytochrome". In: Nature communications 9.1 (2018), p. 4762. ISSN: 20411723. DOI: $10.1038 / \mathrm{s} 41467-018-07246-2$.

[66] B. Karniol et al. "Phylogenetic analysis of the phytochrome superfamily reveals distinct microbial subfamilies of photoreceptors". In: The Biochemical journal 392.Pt 1 (2005), pp. 103-116. DOI: 10.1042/BJ20050826.

[67] M. G. Khrenova, A. M. Kulakova, and A. V. Nemukhin. "Competition between two cysteines in covalent binding of biliverdin to phytochrome domains". In: Organic \& biomolecular chemistry 16.40 (2018), pp. 7518-7529.

[68] Klinische Hepatologie. Springer Berlin Heidelberg, 2003.

[69] T. Konen. "One-step RESOLFT with a positively switchable RSFP with improved deactivation kinetics". PhD Thesis. 2019.

[70] D. E. Koppel, C. Carlson, and H. Smilowitz. "Analysis of heterogeneous fluorescence photobleaching by video kinetics imaging: the method of cumulants". In: Journal of microscopy 155.2 (1989), pp. 199-206.

[71] J. C. Lagarias and H. Rapoport. "Chromopeptides from phytochrome. The structure and linkage of the PR form of the phytochrome chromophore". In: Journal of the American Chemical Society 102.14 (1980), pp. 4821-4828. ISSN: 0002-7863. DOI: 10.1021/ja00534a042.

[72] P. Lamesch et al. “hORFeome v3.1: A resource of human open reading frames representing over 10,000 human genes". In: Genomics 89.3 (2007), pp. 307-315. ISSN: 0888-7543. DOI: 10.1016/j · ygeno. 2006.11.012.

[73] T. Lamparter et al. "The biliverdin chromophore binds covalently to a conserved cysteine residue in the N-terminus of Agrobacterium phytochrome Agp1". In: Biochemistry 43.12 (2004), pp. 3659-3669. ISSN: 0006-2960. DOI: 10. 1021/bi0356931.

[74] M. Lasagna et al. "Apohorseradish peroxidase unfolding and refolding: intrinsic tryptophan fluorescence studies". In: Biophysical journal 76.1 (1999), pp. 443-450.

[75] F Liu, Y. Song, and D Liu. "Hydrodynamics-based transfection in animals by systemic administration of plasmid DNA". In: Gene therapy 6.7 (1999), pp. 1258-1266.

[76] G. Lukinavičius et al. "Fluorescent dyes and probes for super-resolution microscopy of microtubules and tracheoles in living cells and tissues". In: Chem. Sci. 9.13 (2018), pp. 3324-3334. ISSN: 2041-6520. DOI: 10.1039/c7sc05334g.

[77] M Chalfie, Y Tu, G Euskirchen, WW Ward, DC Prasher. "Green fluorescent protein as a marker for gene expression". In: Science (1994), pp. 802-805. ISSN: 0036-8075.

[78] M. D. Maines. "Biliverdin reductase: PKC interaction at the cross-talk of MAPK and PI3K signaling pathways". In: Antioxidants \& redox signaling 9.12 (2007), pp. 2187-2196.

[79] P. Manna et al. "Directed evolution of excited state lifetime and brightness in FusionRed using a microfluidic sorter". In: Integrative Biology 10.9 (2018), pp. 516-526. 
[80] M. E. Matlashov et al. "A set of monomeric near-infrared fluorescent proteins for multicolor imaging across scales". In: Nature Communications 11.1 (2020), p. 123. DOI: 10.1038/s41467-019-13897-6.

[81] M. Mikhaylova et al. "Resolving bundled microtubules using anti-tubulin nanobodies". In: Nature communications 6.1 (2015), pp. 1-7.

[82] M Minsky. "Microscopy apparatus US patent 3013467". In: USP Office, Ed. US (1961).

[83] M. Minsky. "Memoir on inventing the confocal scanning microscope". In: Scanning 10.4 (1988), pp. 128-138.

[84] K Miura et al. "ImageJ Plugin CorrectBleach V2. 0.2". In: Zenodo. doi 10 (2014).

[85] J. R. Moffitt, C. Osseforth, and J. Michaelis. "Time-gating improves the spatial resolution of STED microscopy". In: Optics express 19.5 (2011), pp. 4242-4254.

[86] K. Movahedi et al. "Nanobody-based targeting of the macrophage mannose receptor for effective in vivo imaging of tumor-associated macrophages". In: Cancer research 72.16 (2012), pp. 4165-4177.

[87] S. Nagano. "From photon to signal in phytochromes: similarities and differences between prokaryotic and plant phytochromes". In: Journal of plant research 129.2 (2016), pp. 123-135.

[88] B. Nöding, H. Herrmann, and S. Köster. "Direct observation of subunit exchange along mature vimentin intermediate filaments". In: Biophysical journal 107.12 (2014), pp. 2923-2931.

[89] O. Nureki et al. "An enzyme with a deep trefoil knot for the active-site architecture". In: Acta Crystallographica Section D: Biological Crystallography 58.7 (2002), pp. 1129-1137.

[90] O. S. Oliinyk et al. "Smallest near-infrared fluorescent protein evolved from cyanobacteriochrome as versatile tag for spectral multiplexing". In: Nature communications 10.1 (2019), p. 279. ISSN: 2041-1723. DOI: 10 . 1038/s41467018-08050-8.

[91] J. Oracz et al. "Photobleaching in STED nanoscopy and its dependence on the photon flux applied for reversible silencing of the fluorophore". In: Scientific reports 7.1 (2017), pp. 1-14.

[92] M. Ormö et al. "Crystal structure of the Aequorea victoria green fluorescent protein". In: Science (New York, N.Y.) 273.5280 (1996), pp. 1392-1395. ISSN: 1095-9203. DOI: 10.1126/science.273.5280.1392.

[93] F. Pennacchietti et al. "Fast reversibly photoswitching red fluorescent proteins for live-cell RESOLFT nanoscopy". In: Nature methods 15.8 (2018), pp. 601604.

[94] K. D. Piatkevich, F. V. Subach, and V. V. Verkhusha. "Engineering of bacterial phytochromes for near-infrared imaging, sensing, and light-control in mammals". In: Chemical Society reviews 42.8 (2013), pp. 3441-3452. ISSN: 1460-4744. DOI: $10.1039 / \cos 35458 j$.

[95] T. Pleiner et al. "Nanobodies: site-specific labeling for super-resolution imaging, rapid epitope-mapping and native protein complex isolation". In: Elife 4 (2015), e11349.

[96] A. M. Porcelli et al. " $\mathrm{pH}$ difference across the outer mitochondrial membrane measured with a green fluorescent protein mutant". In: Biochemical and biophysical research communications 326.4 (2005), pp. 799-804. 
[97] D. C. Prasher et al. "Primary structure of the Aequorea victoria green-fluorescent protein". In: Gene 111.2 (1992), pp. 229-233. ISSN: 03781119. DOI: 10 . 1016 / 0378-1119(92) 90691-H.

[98] S. von Prowazek. “Fluorescenz der Zellen". In: Zoologischer Anzeiger 42 (1913), pp. 374-380.

[99] P. H. Quail. "An emerging molecular map of the phytochromes". In: Plant, Cell \& Environment 20.6 (1997), pp. 657-665. ISSN: 1365-3040. DOI: $10.1046 /$ j.1365-3040.1997.d01-108.x.

[100] P. H. Quail. "Phytochrome photosensory signalling networks". In: Nature reviews. Molecular cell biology 3.2 (2002), pp. 85-93. ISSN: 1471-0072. DOI: 10 . $1038 / \mathrm{nrm} 728$.

[101] M. Ratz. “CRISPR-Cas9-mediated protein tagging in human cells for RESOLFT nanoscopy and the analysis of mitochondrial prohibitins". PhD Thesis. 2015.

[102] M. Ratz et al. “CRISPR/Cas9-mediated endogenous protein tagging for RESOLFT super-resolution microscopy of living human cells". In: Scientific reports 5 (2015), p. 9592. ISSN: 2045-2322. DOI: 10.1038/srep09592.

[103] L. Rayleigh. "On the theory of optical images, with special reference to the microscope". In: Journal of the Royal Microscopical Society 23.4 (1903), pp. 447473.

[104] B. G. Reid and G. C. Flynn. "Chromophore formation in green fluorescent protein". In: Biochemistry 36.22 (1997), pp. 6786-6791. ISSN: 0006-2960. DOI: 10.1021/bi970281w.

[105] J. Riedl et al. "Lifeact: a versatile marker to visualize F-actin". In: Nature methods 5.7 (2008), pp. 605-607.

[106] J. P. Rigaut and J. Vassy. "High-resolution three-dimensional images from confocal scanning laser microscopy. Quantitative study and mathematical correction of the effects from bleaching and fluorescence attenuation in depth." In: Analytical and quantitative cytology and histology 13.4 (1991), pp. 223-232.

[107] E Rittweger et al. "Fluorescence depletion mechanisms in super-resolving STED microscopy". In: Chemical physics letters 442.4-6 (2007), pp. 483-487.

[108] N. C. Rockwell, Y.-S. Su, and J. C. Lagarias. "Phytochrome structure and signaling mechanisms". In: Annu. Rev. Plant Biol. 57 (2006), pp. 837-858.

[109] E. A. Rodriguez et al. "A far-red fluorescent protein evolved from a cyanobacterial phycobiliprotein". In: Nature methods 13.9 (2016), pp. 763-769. ISSN: 1548-7105. DOI: 10.1038/nmeth. 3935.

[110] O. C. Rogers, D. M. Johnson, and E. Firnberg. "mRhubarb: Engineering of monomeric, red-shifted, and brighter variants of iRFP using structure-guided multi-site mutagenesis". In: Scientific reports 9.1 (2019), p. 15653. ISSN: 20452322. DOI: $10.1038 / \mathrm{s} 41598-019-52123-7$.

[111] K. A. Rumyantsev et al. "Design of near-infrared single-domain fluorescent protein GAF-FP based on bacterial phytochrome". In: Cell and Tissue Biology 11.1 (2017), pp. 16-26. DOI: 10.1134/S1990519X17010102.

[112] M. J. Rust, M. Bates, and X. Zhuang. "Sub-diffraction-limit imaging by stochastic optical reconstruction microscopy (STORM)". In: Nature methods 3.10 (2006), pp. 793-796. 
[113] Y. Saito et al. "Machine-learning-guided mutagenesis for directed evolution of fluorescent proteins". In: ACS synthetic biology 7.9 (2018), pp. 2014-2022.

[114] A. Sawano and A. Miyawaki. "Directed evolution of green fluorescent protein by a new versatile PCR strategy for site-directed and semi-random mutagenesis". In: Nucleic Acids Research 28.16 (2000), e78. ISSN: 0305-1048.

[115] E. Schäfer and F. Nagy. Photomorphogenesis in plants and bacteria: function and signal transduction mechanisms. Springer Science \& Business Media, 2006.

[116] J. Schindelin et al. "Fiji: an open-source platform for biological-image analysis". In: Nature methods 9.7 (2012), pp. 676-682. ISSN: 1548-7105. DOI: 10 . 1038/nmeth. 2019.

[117] W. M. Schluchter and A. N. Glazer. "Characterization of Cyanobacterial Biliverdin Reductase". In: Journal of Biological Chemistry 272.21 (1997), pp. 1356213569.

[118] D. M. Shcherbakova and V. V. Verkhusha. "Near-infrared fluorescent proteins for multicolor in vivo imaging". In: Nature methods 10.8 (2013), pp. 751-754. ISSN: 1548-7105. DOI: 10.1038/nmeth . 2521.

[119] D. M. Shcherbakova et al. "Bright monomeric near-infrared fluorescent proteins as tags and biosensors for multiscale imaging". In: Nature communications 7 (2016), p. 12405. ISSN: 2041-1723. DOI: 10.1038/ncomms 12405.

[120] D. M. Shcherbakova et al. "Natural photoreceptors as a source of fluorescent proteins, biosensors, and optogenetic tools". In: Annual review of biochemistry 84 (2015), pp. 519-550. ISSN: 1545-4509. DOI: 10 . 1146 / annurev-biochem060614-034411.

[121] A. A. Shemetov, O. S. Oliinyk, and V. V. Verkhusha. "How to Increase Brightness of Near-Infrared Fluorescent Proteins in Mammalian Cells". In: Cell chemical biology 24.6 (2017), 758-766.e3. ISSN: 2451-9456. DOI: 10 . 1016/j . chembiol. 2017.05 .018$.

[122] O. Shimomura, F. H. Johnson, and Y. Saiga. "Extraction, purification and properties of aequorin, a bioluminescent protein from the luminous hydromedusan, Aequorea". In: Journal of Cellular and Comparative Physiology 59.3 (1962), pp. 223-239. ISSN: 1553-0809. DOI: 10.1002/jcp. 1030590302.

[123] X. Shu et al. "Mammalian expression of infrared fluorescent proteins engineered from a bacterial phytochrome". In: Science (New York, N.Y.) 324.5928 (2009), pp. 804-807. ISSN: 1095-9203. DOI: 10.1126/science.1168683.

[124] H. Smith. "Phytochromes and light signal perception by plants-an emerging synthesis". In: Nature 407.6804 (2000), pp. 585-591. ISSN: 1476-4687. DOI: 10. 1038/35036500.

[125] P. Soellner, R. A. Quinlan, and W. W. Franke. "Identification of a distinct soluble subunit of an intermediate filament protein: tetrameric vimentin from living cells". In: Proceedings of the National Academy of Sciences 82.23 (1985), pp. 7929-7933.

[126] J. Soret. "Analyse spectrale: Sur le spectre d'absorption du sang dans la partie violette et ultra-violette". In: Compt. Rend 97 (1883), pp. 1269-1270.

[127] O. V. Stepanenko et al. "A knot in the protein structure-probing the nearinfrared fluorescent protein i RFP designed from a bacterial phytochrome". In: The FEBS journal 281.9 (2014), pp. 2284-2298. 
[128] A. C. Steven et al. "The distribution of mass in heteropolymer intermediate filaments assembled in vitro. Stem analysis of vimentin/desmin and bovine epidermal keratin." In: Journal of Biological Chemistry 258.13 (1983), pp. 83238329.

[129] U. Sweere et al. "Interaction of the response regulator ARR4 with phytochrome B in modulating red light signaling". In: Science 294.5544 (2001), pp. 11081111.

[130] H. Takala et al. "Light-induced changes in the dimerization interface of bacteriophytochromes". In: Journal of Biological Chemistry 290.26 (2015), pp. 1638316392.

[131] H. Takala et al. "Signal amplification and transduction in phytochrome photosensors". In: Nature 509.7499 (2014), pp. 245-248.

[132] W. R. Taylor. "A deeply knotted protein structure and how it might fold". In: Nature 406.6798 (2000), pp. 916-919.

[133] W. R. Taylor and K. Lin. “Protein knots: a tangled problem”. In: Nature 421.6918 (2003), pp. 25-25.

[134] M. J. Terry. "Biosynthesis and Analysis of Bilins". In: Heme, chlorophyll, and bilins. Ed. by A. G. Smith and M. Witty. Vol. 0. Totowa, N.J.: Humana Press, 2002, pp. 273-291. ISBN: 978-1-59259-243-2. DOI: 10 . 1385/1-59259-243-0 : 273.

[135] The Digestive System. Elsevier, 2010. ISBN: 9780702033674.

[136] A. T. Ulijasz et al. "Characterization of two thermostable cyanobacterial phytochromes reveals global movements in the chromophore-binding domain during photoconversion". In: Journal of Biological Chemistry 283.30 (2008), pp. 2125121266.

[137] G. Vicidomini et al. "STED with wavelengths closer to the emission maximum". In: Optics express 20.5 (2012), pp. 5225-5236.

[138] J. R. Wagner et al. "A light-sensing knot revealed by the structure of the chromophore-binding domain of phytochrome". In: Nature 438.7066 (2005), pp. 325-331.

[139] J. R. Wagner et al. "High resolution structure of Deinococcus bacteriophytochrome yields new insights into phytochrome architecture and evolution". In: Journal of Biological Chemistry 282.16 (2007), pp. 12298-12309.

[140] J. R. Wagner et al. "Mutational analysis of Deinococcus radiodurans bacteriophytochrome reveals key amino acids necessary for the photochromicity and proton exchange cycle of phytochromes". In: Journal of Biological Chemistry 283.18 (2008), pp. 12212-12226.

[141] W. W. Ward et al. "Spectral perturbations of the Aequorea green-fluorescent protein". In: Photochemistry and photobiology 35.6 (1982), pp. 803-808.

[142] R. Weissleder. "A clearer vision for in vivo imaging". In: Nature biotechnology 19.4 (2001), pp. 316-317.

[143] L. J. van Wilderen et al. “Mid-Infrared Picosecond Pump- Dump- Probe and Pump- Repump-Probe Experiments to Resolve a Ground-State Intermediate in Cyanobacterial Phytochrome Cph1". In: The Journal of Physical Chemistry B 113.51 (2009), pp. 16354-16364. 
[144] S. I. Woodard and H. A. Dailey. "Regulation of heme biosynthesis in Escherichia coli". In: Archives of Biochemistry and Biophysics 316.1 (1995), pp. 110115.

[145] C. A. Wurm et al. "Novel red fluorophores with superior performance in STED microscopy". In: Optical Nanoscopy 1.1 (2012), p. 7.

[146] D. Wüstner et al. "Photobleaching kinetics and time-integrated emission of fluorescent probes in cellular membranes". In: Molecules 19.8 (2014), pp. 1109611130.

[147] F. Yang, L. G. Moss, and G. N. Phillips. "The molecular structure of green fluorescent protein". In: Nature Biotechnology 14.10 (1996), pp. 1246-1251. ISSN: 1546-1696. DOI: 10.1038/nbt1096-1246.

[148] X. Yang, J. Kuk, and K. Moffat. "Conformational differences between the Pfr and Pr states in Pseudomonas aeruginosa bacteriophytochrome". In: Proceedings of the National Academy of Sciences 106.37 (2009), pp. 15639-15644.

[149] X. Yang, J. Kuk, and K. Moffat. "Crystal structure of Pseudomonas aeruginosa bacteriophytochrome: photoconversion and signal transduction". In: Proceedings of the National Academy of Sciences 105.38 (2008), pp. 14715-14720.

[150] X. Yang et al. "Light signaling mechanism of two tandem bacteriophytochromes". In: Structure 23.7 (2015), pp. 1179-1189.

[151] D. Yu et al. "A naturally monomeric infrared fluorescent protein for protein labeling in vivo". In: Nature methods 12.8 (2015), pp. 763-765. ISSN: 1548-7105. DOI: $10.1038 /$ nmeth. 3447.

[152] D. Yu et al. "An improved monomeric infrared fluorescent protein for neuronal and tumour brain imaging". In: Nature communications 5 (2014), p. 3626. ISSN: 2041-1723. DOI: $10.1038 /$ ncomms 4626.

[153] T. I. Zarembinski et al. “Deep trefoil knot implicated in RNA binding found in an archaebacterial protein". In: Proteins: Structure, Function, and Bioinformatics 50.2 (2003), pp. 177-183.

[154] L. Zhang et al. "Reaction progress of chromophore biogenesis in green fluorescent protein". In: Journal of the American Chemical Society 128.14 (2006), pp. 4766-4772. ISSN: 1520-5126. DOI: $10.1021 /$ ja0580439. 